\title{
The invisible body
}

Citation for published version (APA):

Zhan, M. (2018). The invisible body: the neural mechanisms of non-conscious and conscious processing of emotional bodies. [Doctoral Thesis, Maastricht University]. Ipskamp Printing BV. https://doi.org/10.26481/dis.20180516mz

Document status and date:

Published: 01/01/2018

DOI:

$10.26481 /$ dis.20180516mz

Document Version:

Publisher's PDF, also known as Version of record

\section{Please check the document version of this publication:}

- A submitted manuscript is the version of the article upon submission and before peer-review. There can be important differences between the submitted version and the official published version of record.

People interested in the research are advised to contact the author for the final version of the publication, or visit the DOI to the publisher's website.

- The final author version and the galley proof are versions of the publication after peer review.

- The final published version features the final layout of the paper including the volume, issue and page numbers.

Link to publication

\footnotetext{
General rights rights.

- You may freely distribute the URL identifying the publication in the public portal. please follow below link for the End User Agreement:

www.umlib.nl/taverne-license

Take down policy

If you believe that this document breaches copyright please contact us at:

repository@maastrichtuniversity.nl

providing details and we will investigate your claim.
}

Copyright and moral rights for the publications made accessible in the public portal are retained by the authors and/or other copyright owners and it is a condition of accessing publications that users recognise and abide by the legal requirements associated with these

- Users may download and print one copy of any publication from the public portal for the purpose of private study or research.

- You may not further distribute the material or use it for any profit-making activity or commercial gain

If the publication is distributed under the terms of Article $25 \mathrm{fa}$ of the Dutch Copyright Act, indicated by the "Taverne" license above, 
The Invisible Body Minye Zhan 
(C) Minye Zhan, Maastricht, 2018

All rights reserved.

No part of this publication may be reproduced, stored in a retrieval system or transmitted in any form or by any means, electronic, mechanical, photocopying, recording or otherwise, without prior written permission of the author.

Cover inspired by the novel The Invisible Man (H. G. Wells, 1897). (C @JaneMere Cover font: Christophe Plantin, 1579

Production: Ipskamp Printing

ISBN: 978-94-028-1042-4 


\section{Propositions}

- The perception of both human bodies and emotion involves distributed brain areas.

- The phenomenon of visual awareness is multifaceted, involving a variety of brain areas, and mechanisms at different processing levels.

- The dorsal pathway areas are less associated with visual awareness, comparing to the ventral pathway areas under CFS.

- The mechanism of CFS may relate to the rhythmic attentional sampling mechanism, operating at theta frequencies.

- Each methodological tool has its limitations (including CFS, 3T and 7T fMRI, univariate and multivariate analyses).

- Know your data.

- It is a capital mistake to theorize before one has data. Insensibly one begins to twist facts to suit theories, instead of theories to suit facts. - Arthur Conan Doyle (1892). A Scandal in Bohemia, in The Adventures of Sherlock Holmes.

- The general public should be updated with the most recent research of consciousness (and brain science in general).

- The devil is in the detail. - Proverb.

- 三思而后行. Think thrice before you act. - Confucius, Analects.

- 工欲善其事, 必先利其器. The mechanic who wishes to do his work well, must first sharpen his tools. - Confucius, Analects. 



\title{
The invisible body
}

\section{The neural mechanisms of non-conscious and conscious processing of emotional bodies}

\author{
DISSERTATION \\ TO OBTAIN THE DEGREE OF DOCTOR AT \\ MAASTRICHT UNIVERSITY ON THE AUTHORITY OF THE RECTOR MAGNIFICUS \\ PROF.DR. RIANNE M. LETSCHERT \\ IN ACCORDANCE WITH THE DECISION OF THE BOARD OF DEANS, \\ TO BE DEFENDED IN PUBLIC \\ ON WEDNESDAY $16^{\text {TH }}$ OF MAY 2018 AT 14.00 HOURS
}

BY

MINYE ZHAN 


\section{Supervisors:}

Prof. dr. Beatrice de Gelder

Prof. dr. Rainer Goebel

\section{Assessment Committee:}

Prof. dr. P. de Weerd (Chair)

Prof. dr. J. Culham (Western University, Canada)

Prof. dr. E. Formisano

Prof. dr. A. Sack

dr. M. Tamietto (Tilburg University, Netherlands; Università di Torino, Italy) 


\section{Contents}

$\begin{array}{llr}\text { Chapter } 1 & \text { Introduction }\end{array}$

$\begin{array}{lll}\text { Chapter } 2 & \text { The body as a tool for anger awareness - Differential } & 27\end{array}$

effects of angry facial and bodily expressions on

suppression from awareness

Chapter $3 \quad$ Continuous flash suppression induces strong suppression of 43

visual stimulus information

Chapter $4 \quad$ Influence of continuous flash suppression mask frequency 69

on stimulus visibility

Chapter $5 \quad$ Ventral and dorsal pathways differently related to visual

awareness of body postures under continuous flash

suppression

Chapter 6 Action categories are represented in a large distributed

network in the brain - evidence from 7T fMRI

Chapter 7 The influence of a short domestic-violence VR scenario on

emotional face and body perception

Chapter $8 \quad$ General discussion

Valorization

Acknowledgments

Publications

Curriculum Vitae 

Chapter 1

Introduction 


\section{The research of visual processing in the human brain: the two-stream model}

The understanding of the visual processing and consciousness in the human brain has been advanced significantly by lesion studies in human patients and monkeys, before the advent of functional brain imaging. Several types of patients have been extensively studied with behavioral experiments, showing several double dissociations between object recognition, online action performance, and visual awareness. For visual agnosia patients caused by occipito-temporal lesions, their ability to perceive and recognize objects has been damaged, but their ability to grasp an object online is intact (James, Culham, Humphrey, Milner, \& Goodale, 2003); for patients with optic ataxia caused by posterior parietal lesions, they do not have difficulties in recognizing objects, but their ability to point, reach, and grasp contralesional objects online are impaired (Jakobson, Archibald, Carey, \& Goodale, 1991, for a review see Andersen, Andersen, Hwang, \& Hauschild, 2014). For blindsight patients due to lesions of their primary visual areas (V1), they do not have subjective awareness of the stimuli presented in their blind visual field, but nevertheless could react to them with abovechance accuracy (Weiskrantz, 1986), and could even avoid obstacles when the lesion involved bilateral V1 (patient T.N., de Gelder et al., 2008); For neglect patients with parietal lesions, their object recognition and obstacle avoidance behavior are intact (Mclntosh, McClements, Dijkerman, Birchall, \& Milner, 2004), but they often lose visual awareness of their contra-lesional visual field (Driver \& Vuilleumier, 2001, for reviews see Corbetta \& Shulman, 2011; Driver \& Mattingley, 1998).

The evidence from patient studies had led to the establishment of the two-stream model for visual processing beyond V1 (Milner \& Goodale, 2006, 2008). The ventral (occipito-temporal) areas are responsible for visual shape processing and recognition of objects, where the information could be utilized after a delay. The dorsal (parietal) areas are responsible for online visual-spatial guidance of actions, which is an automatic process largely independent of awareness, and the information could only be utilized on a moment-to-moment basis. For example, the visual agnosia patient D.F. demonstrated normal online action for grasping an object, but this normal performance was disrupted after a delay as short as $2 \mathrm{~s}$ (Goodale, Jakobson, \& Keillor, 1994), highlighting the moment-to-moment nature of dorsal processing.

Although patient studies offer direct causal evidence for brain functions, the cases of patients are rare; the lesioned sites are also heterogeneous, and involve compensation mechanisms and reorganizations of the brain networks. For example, fiber connections observed under diffusion tensor imaging (DTI) for the blindsight patient G.Y. showed substantial modification, and even novel pathways that were not detected in healthy control participants (Bridge, Thomas, Jbabdi, \& Cowey, 2008; M. Tamietto, Pullens, de Gelder, Weiskrantz, \& Goebel, 2012). Thus, much research is performed with healthy participants, with behavioral, neuroimaging, and non-invasive stimulation techniques.

\section{Functions in the ventral stream}


With the advent of functional magnetic imaging (fMRI) and the development of cognitive subtraction under the univariate general linear model analysis (Friston et al., 1994), researchers have been mapping out category-specific areas, by contrasting the blood oxygenation level-dependent (BOLD) activity between the intact stimuli of object categories and the scrambled stimuli images.

Multiple category/attribute-specific areas have been localized in the ventral stream in human participants. Along the occipito-temporal lobes, these areas include: the lateral occipital (LO) areas for objects (Malach et al., 1995); the fusiform face area (FFA) for faces, in the fusiform gyri (Kanwisher, McDermott, \& Chun, 1997); the parahippocampal place area (PPA) for places and buildings, medial and anterior to the FFA (Epstein \& Kanwisher, 1998); the visual word form area (VWFA) for written words, lateral to the FFA (Cohen et al., 2000); and the visual area 4 (V4) for color processing, lying posterior to the fusiform gyrus

(McKeefry \& Zeki, 1997). See Figure 1. These areas have large visual receptive fields, and are largely invariant to the size, retinotopic location, viewing angle of the stimuli. In the ventral stream, the visual form information of a stimulus is thought to undergo a hierarchical process, with increasingly abstract information being extracted in higher-level areas (GrillSpector \& Malach, 2004; Grill-Spector \& Weiner, 2014). Multiple organizational rules have been proposed for the representation of different object categories, including the eccentricity of the object category under normal viewing conditions (Malach, Levy, \& Hasson, 2002), real object size (Konkle \& Oliva, 2012), and the animacy/inanimacy of the object (Kiani, Esteky, Mirpour, \& Tanaka, 2007; Kriegeskorte et al., 2008).

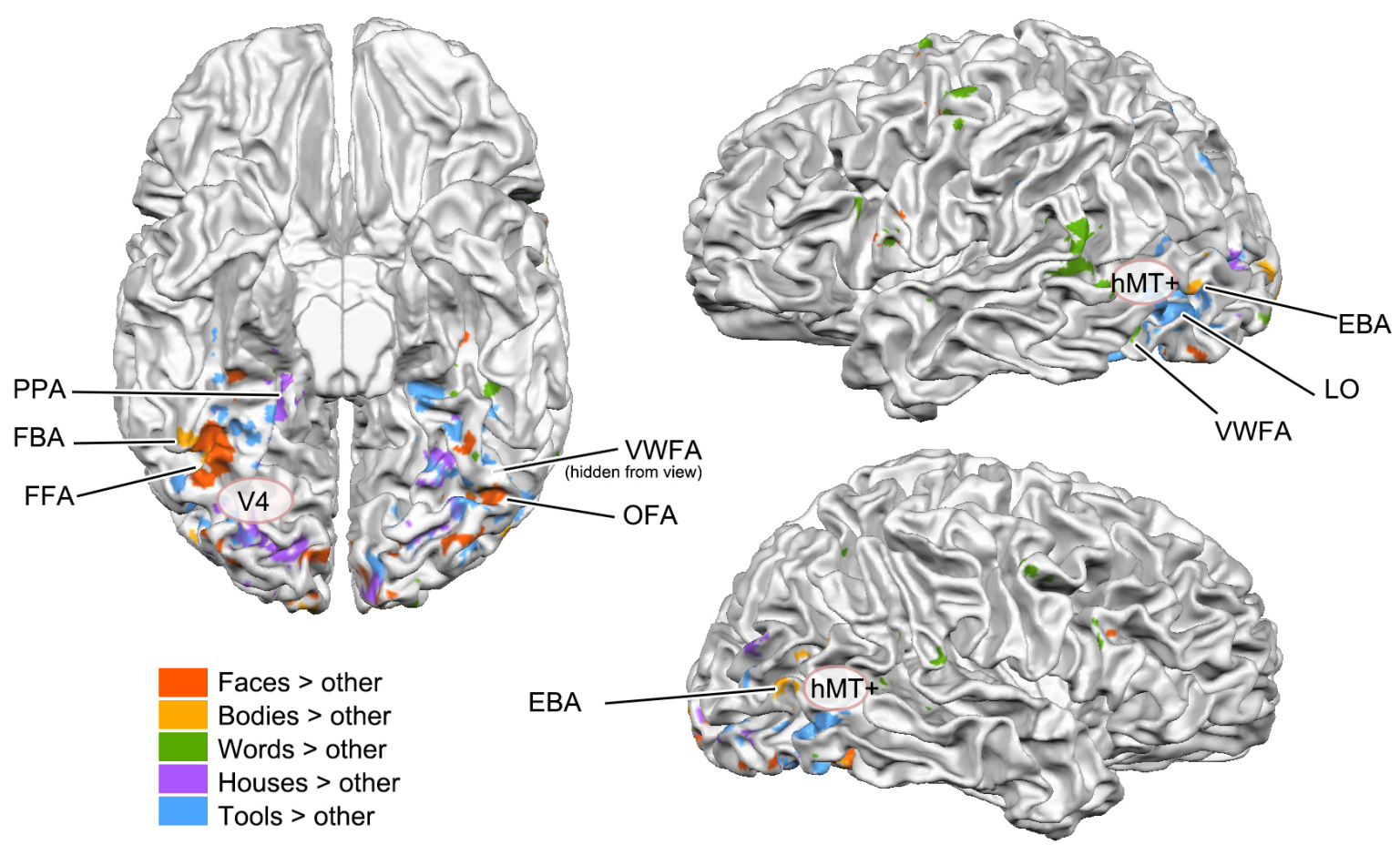

Figure 1. Category-specific areas in the ventral-lateral areas. The contrast maps were from the functional localizer data of one participant (data from Chapter 6, 7T, resolution $1.2 \mathrm{~mm}$ isotropic, smoothed with $3 \mathrm{~mm}$ FWHM, p=.001 uncorrected), mapped on the cortical surface of the same participant. The maps for each object category were obtained by contrasting the category by the 


\section{Chapter 1}

other 4 categories. For tools not much activation along the intraparietal sulcus (IPS) is observed due to surface sampling, and also because this contrast assumes specificity for the category (Compare to Figure $\mathbf{2}$ in Chapter $\mathbf{5}$ which doesn't assume strict specificity). The V4 and hMT+ were indicated with ellipses, in the locations roughly corresponding to those in the literature. No claim of laterality is assumed in the current figure. Abbreviations: PPA: parahippocampal place area; FBA: fusiform body area; FFA: fusiform face area; VWFA: visual word form area; OFA: occipital face area; LO: lateral occipital area; EBA: extrastriate body area; hMT+: human middle temporal complex.

Within these object categories, the human faces have been studied most extensively. The faces show an inversion effect in healthy participants, that the inverted faces were processed less optimally than upright ones, thought to be due to a disruption of a configural/holistic processing for faces (Farah, Tanaka, \& Drain, 1995). The inversion effect is reflected in healthy participants by longer reaction times (RTs) behaviorally, and lower BOLD activity in the FFA (Yovel \& Kanwisher, 2005). In developmental and acquired prosopagnosic participants, this inversion effect was not present or was even reversed (de Gelder \& Rouw, 2000; Farah, Wilson, Drain, \& Tanaka, 1995). In monkey fMRI studies, patches sensitive to faces were found in the superior temporal sulcus (STS), which are thought to be homologous to the human face-sensitive areas (Tsao, Freiwald, Knutsen, Mandeville, \& Tootell, 2003). In a study of monkey single-cell recordings, face selectivity was observed for $97 \%$ of the cells recorded in an fMRI-activated patch in STS that corresponded to human FFA ("selectivity" defined by responses to faces more than twice strongly than non-face stimuli, Tsao, Freiwald, Tootell, \& Livingstone, 2006). However the specificity of FFA for faces in human participants measured by fMRI is not absolute (e.g. see Tsao et al., 2003), and is still under debate (Gauthier, Skudlarski, Gore, \& Anderson, 2000; Yovel \& Kanwisher, 2004). Apart from the FFA in human participants, two other areas also exhibit face selectivity: the occipital face area (OFA, Clark et al., 1996; Kanwisher et al., 1997) and the posterior superior temporal sulcus (pSTS, Kanwisher et al., 1997),

\section{Functions in the dorsal stream}

Comparing to the ventral stream, the functions in the dorsal stream are more complex and less specific for object categories. An exception is the tool category (Johnson-Frey, 2004), although it is still under debate whether the dorsal stream is sensitive to the tools as a category, or to the elongated shapes of the tools (Almeida, Mahon, Nakayama, \& Caramazza, 2008; Sakuraba, Sakai, Yamanaka, Yokosawa, \& Hirayama, 2012).

In the dorsal stream, several areas along the intraparietal sulcus (IPS) shown by fMRI are involved in the pointing, reaching and grasping actions, including the medial IPS (mIPS), and the anterior IPS (alPS) (Culham, Cavina-Pratesi, \& Singhal, 2006; Culham \& Valyear, 2006). These two areas are in a larger network that is activated during action observation, termed the action observation network (AON), which in addition includes extrastriate visual areas near the hMT+, the inferior parietal lobule (IPL), the dorsal and ventral premotor areas (PMd, PMd), and inferior frontal areas (Caspers, Zilles, Laird, \& Eickhoff, 2010; Cross, Kraemer, 
Hamilton, Kelley, \& Grafton, 2009; Grafton \& Hamilton, 2007). See Figure 2. In monkey studies, neurons in premotor and parietal areas homologous to the human AON areas fired both when observing and performing an action, thus the network was also named mirror neuron networks, and was thought to carry out an important mechanism in understanding actions performed by others (Rizzolatti \& Craighero, 2004; Rizzolatti \& Sinigaglia, 2010).

Comparing to the mIPS and aIPS, the posterior IPS (pIPS) is more involved in the control of saccadic movements and spatial attention (Culham et al., 2006; Culham \& Valyear, 2006). It is thought to be a node in a larger attentional network, which could be divided into a dorsal and a ventral system (Corbetta, Patel, \& Shulman, 2008). The dorsal attention system includes IPS, superior parietal lobule, and FEF, and guides spatial attention to the stimulus in a top-down manner; the ventral attention system is more lateralized in the right hemisphere, including areas close to the temporo-parietal junction (TPJ), the middle frontal gyrus (MFG), the ventral frontal cortex, and the anterior insula. The ventral network guides the spatial attention by behaviorally relevant stimuli (Corbetta et al., 2008). See Figure 2.

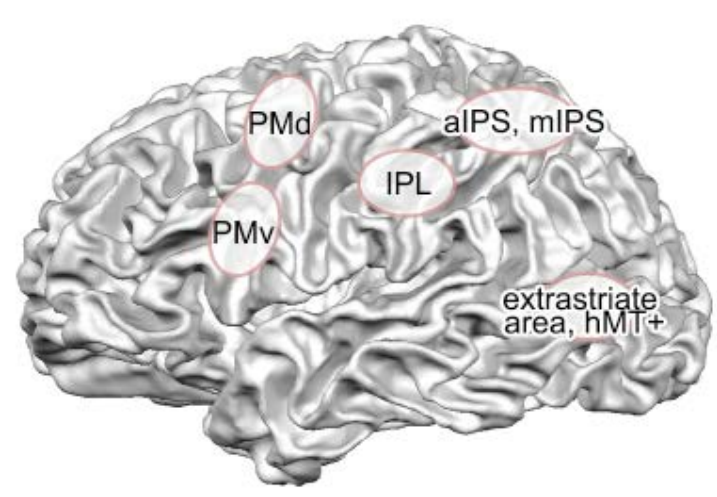

The action observation network

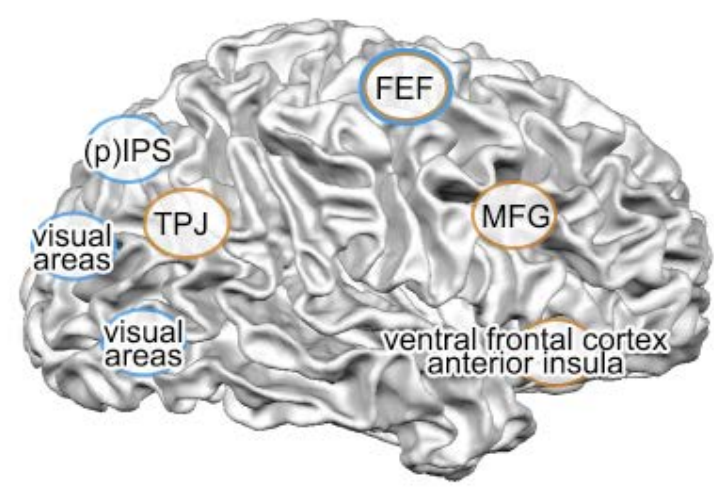

The dorsal and ventral attention networks

Figure 2. Schematic representation of the networks involving dorsal areas. The action observation network is plotted on the left hemisphere, adapted from Figure 1 of Caspers et al., 2010. The dorsal (blue) and ventral (yellow) attentional networks are plotted on the right hemisphere, adapted from Figure 2 of Corbetta et al., 2008. The laterality of the networks are not represented I the current figure. Abbreviations: hMT+: human middle temporal complex; IPS: intraparietal sulcus; IPL: inferior parietal lobule; PMd/PMv: dorsal/ventral premotor cortices; TPJ: temporo-parietal junction; FEF: frontal eye field; MFG: middle frontal gyrus.

The dorsal stream is also responsible to combining visual inputs from the two eyes, thought to be combined between V1 and V2 (Barendregt, Harvey, Rokers, \& Dumoulin, 2015), and form 3D representations for objects. For perceiving 3D shapes through binocular disparity cues, activation was found in the dorsal pathway, from V2/V3 along the IPS to the aIPS, and in PMv (Orban, 2011), which includes most of the dorsal areas important for observing and performing actions. In the V3a complex and the V7, the fMRI activity measured was found to 


\section{Chapter 1}

be correlated with the subjectively rated depth in individual participants (Georgieva, Peeters, Kolster, Todd, \& Orban, 2009).

Starting from the V1 and through the dorsal stream, the visual representations are thought to undergo a transformation of coordinates (Buneo \& Andersen, 2006), from a retinotopic coordinate to eye, head, and hand coordinates. Along with these transformations, multisensory (visual, tactile, auditory) representations of peripersonal spaces are centered on body parts, including the head, the body and the hands, the mapping of which are guided by visual inputs, and could be dynamically changed after active tool use (di Pellegrino \& Ladavas, 2015). Areas that map the peripersonal space include IPS, PMd and PMv (Culham et al., 2006; di Pellegrino \& Ladavas, 2015). As stimulating the putative homologous VIP (corresponding to human mIPS/dDIPSA, Orban, 2016) and polysensory zone (monkey $\mathrm{PMv} / \mathrm{F} 4$, corresponding to human PMv) induced defense-like movements in monkeys, these areas are thought to be involved in avoidance and defensive behaviors (Graziano \& Cooke, 2006).

In monkeys, the latencies of the dorsal areas in general are shorter than the ones of the ventral (temporal) areas, due to magnocellular inputs and direct projections from V1 bypassing intermediate areas. Ventral areas on the other hand receive mainly parvocellular inputs, and the information were relayed in a more serial and hierarchical fashion (Nowak \& Bullier, 1997). In humans, the dorsal and ventral streams are less segregated than in monkeys, with more connections between dorsal and ventral areas (Zilles \& Clarke, 1997).

\section{The conscious and non-conscious processing of (facial) emotions}

The emotional and social information, being behaviorally relevant in our everyday life, enjoys privileged processing under both conscious and non-conscious conditions. Under conscious processing, evidence abounds that emotional stimuli show attention-grabbing effects comparing to non-emotional stimuli, under behavioral paradigms such as emotional stroop, visual search, cueing (for a review see Yiend, 2010). ALE meta-analyses show that observing emotional expressions induce activations in cortical areas including pSTS, temporal pole, inferior frontal cortex (IFC), insula, orbitofrontal cortex (OFC), posterior cingulate, pre-supplementary motor area (preSMA), anterior cingulate cortex (ACC), dorsal and ventral medial prefrontal cortices (dmPFC, vmPFC), and also in subcortical areas including amygdala, ventral striatum, thalamus, hypothalamus, and periaqueductal gray (Dricu \& Fruhholz, 2016; Kober et al., 2008). See Figure 3. 


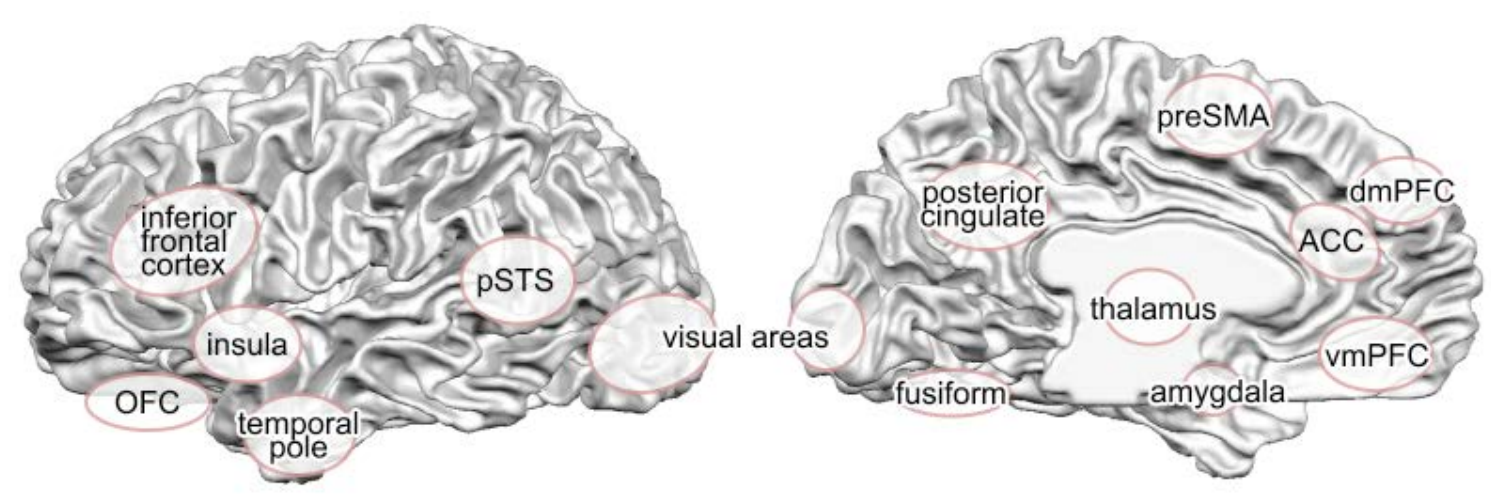

Figure 3. Brain areas activated in emotional experiments, shown in ALE meta-analyses. Figure adapted from Figure 1 of Dricu \& Fruhholz, 2016, and Figure 4 of Kober et al., 2008.

Abbreviations: OFC: orbitofrontal cortex; pSTS: posterior superior temporal sulcus; preSMA: presupplementary motor area; ACC: anterior cingulate cortex; dmPFC: dorsal medial prefrontal cortex.

A large majority of emotional studies used facial expressions as the modality of stimuli. The amygdala has been shown with $\mathrm{fMRI}$ studies to be of special importance for both face (i.e. the eye region, including the eye whites, the pupil size, see Demos, Kelley, Ryan, Davis, \& Whalen, 2008; Whalen et al., 2004) and emotion processing, especially when the emotion is fear. Rodent studies showed that the amygdala is involved in fear conditioning (LeDoux, 2003), subsequently the amygdala was proposed to be a fear module (Ohman \& Mineka, 2001) and has been studied intensively in both animals and humans. Although in more recent views, the amygdala is thought to be linked to a wider range of processes, including rewards, relevance, arousal, and attention (Janak \& Tye, 2015; Pessoa, 2010; Sander, Grafman, \& Zalla, 2003). For its role in emotion processing for human participants, an ALE meta-analysis showed that the amygdala is not only sensitive to negative emotions, but also to positive emotions (Sergerie, Chochol, \& Armony, 2008). For studies using face stimuli, an emphasis was also naturally placed on the emotional modulation in face-specific areas, showing enhanced activity when perceiving emotional faces (for a review see Vuilleumier \& Pourtois, 2007).

Besides the amygdala and the face-specific areas, another important set of areas involving in emotion and social processing are the pSTS, and the TPJ adjacent to/overlapping with it. The PSTS is not only involved in face processing, but is also involved in other functions including the processing of biological motion (Grossman, Battelli, \& Pascual-Leone, 2005), and gaze (Allison, Puce, \& McCarthy, 2000), with the latter conveying important social information. The TPJ is involved in important social functions including understanding other people's thoughts (theory of mind, ToM) and empathy, but it is also involved in the sense of agency which distinguish oneself with the others (for ToM, see e.g. Saxe \& Kanwisher, 2003; for two meta-analysis studies of other functions, see Bzdok et al., 2012; Decety \& Lamm, 2007). Overall, the ToM/empathy network substantially overlaps with the default mode network, which is related to multiple higher-order internal processes (for reviews, see Buckner, Andrews-Hanna, \& Schacter, 2008; Mars et al., 2012). As mentioned earlier (see the previous 


\section{Chapter 1}

section), the TPJ is also an important node in the ventral attention network, responsible to reorient attention from more internal processes to external stimuli (Corbetta et al., 2008). However the anatomical location of the TPJ is not consistently defined across these experiments, and falls around the angular gyrus, the pSTS, and the supramarginal gyrus, although the ToM-related activation seemed to be more posterior than the reorientationrelated activation (for reviews see Igelstrom \& Graziano, 2017; Schurz, Tholen, Perner, Mars, \& Sallet, 2017).

With the advance of MR acquisition technology, higher functional resolution has been increasingly used in studying human brain functions. Under $3 T$ magnetic field strength, the resolution improved from the widely used $3 \mathrm{~mm}$ isotropic or bigger, to below $2 \mathrm{~mm}$ in-plane resolution, although a smaller brain coverage consequently followed (e.g., $1.4 \times 1.4 \times 2 \mathrm{~mm}^{3}$ in Baker et al., 2007; $1.5 \mathrm{~mm}$ isotropic in Weiner \& Grill-Spector, 2011). The use of ultra-high field (7T and above) MRI had boosted both the signal to noise ratio (SNR) and the functional resolution (Ugurbil, 2014), and sub-millimeter functional voxel size had already been applied in studying early sensory cortices at mesoscopic scales (for reviews see Cheng, 2016; De Martino et al., 2017; Dumoulin, Fracasso, van der Zwaag, Siero, \& Petridou, 2017; Schluppeck, Sanchez-Panchuelo, \& Francis, 2017). Although the use of 7T with large brain coverage is scarce for higher-order brain functions e.g. emotion, attempts have been made to examine amygdala activity under emotion discrimination tasks with whole-brain coverage $\left(1.5 \times 1.5 \times 2 \mathrm{~mm}^{3}\right.$ in Sladky et al., 2013).

Apart from the developments of MR acquisition technologies, the fMRI data analysis techniques also have undergone huge developments. Multivariate methods offer higher sensitivity than univariate methods, and could provide information at a sub-voxel scale (for a review see Haynes, 2015), thus have increasingly been shedding light on our understanding of brain functions. While the traditional univariate analysis treats single voxels separately and compares averaged BOLD signals between the voxels, the multivariate methods utilize information across different voxels or time points. These multivariate methods include decoding (being generally termed multivariate pattern analysis, MVPA), which classifies neural responses of stimuli exemplars and predicts the category of novel stimuli exemplars (encoding reverses the relationships between the responses and stimuli, for a review see Naselaris, Kay, Nishimoto, \& Gallant, 2011); and representational similarity analysis (RSA), which construct relationships between stimuli and compare these across-stimuli relationships at multiple levels and data modalities (Kriegeskorte et al., 2008; Nili et al., 2014). With the RSA method, one study found categorical representations of different emotions across face, body and voice modalities in STS and mPFC (Peelen, Atkinson, \& Vuilleumier, 2010), and another study found that mOFC (inferior but close to the location of mPFC in the former study) coded valence across visual and gustatory modalities (Chikazoe, Lee, Kriegeskorte, \& Anderson, 2014). For theories of emotions, there have been continual debates about whether there exist a few "basic emotions" with distinct neural substrates, or the emotions are represented in a distributed manner with sharing neural substrates. Multivariate studies have provided new evidence for these two theories, while emphasizing the importance of using ethological and varied stimuli in modality other than face images. One MVPA study with movie clips found that 6 basic emotions (disgust, fear, happiness, sadness, anger, and surprise) could each be classified above chance, with whole-brain data 


\section{Introduction}

performing much better than data in individual regions of interests (ROIs)(Saarimaki et al., 2016). Another RSA study with short stories found that, in ToM regions (mPFC, TPJ, posterior cingulate, STS) that attribute the emotions of others, the representations of emotions were better described by a model of 38 abstract dimensions, than the basic emotion model or the arousal/valence model (Skerry \& Saxe, 2015). A third multivariate study using music and films found that combining the basic emotions model and the model of dimensions (bad/good, active/passive) better described the neural activity than individual models (Kragel \& LaBar, 2015). All three studies started to reveal the multi-dimensional nature of emotional experiences and processes.

For non-conscious emotion processing, the direct evidence mainly came from studies of patients with brain lesions. In neglect patients, emotional faces were extinguished less than neutral faces, showing an attention-grabbing effect (e.g. Vuilleumier \& Schwartz, 2001), and extinguished fearful faces activated the amygdala similar to consciously perceived fearful faces (Vuilleumier et al., 2002). For blindsight patients (G.Y. and T.N.), they could exhibit above-chance discrimination under 2-alternative forced-choice tasks for emotional faces presented in their blind visual fields (e.g. de Gelder, Vroomen, Pourtois, \& Weiskrantz, 1999, Pegna, Khateb, Lazeyras, \& Seghier, 2005), and exhibit a redundant target effect, a facilitation of reaction times when the stimulus in the blind visual field has congruent emotion information to the stimulus presented in the intact visual field (e.g. de Gelder, Pourtois, van Raamsdonk, Vroomen, \& Weiskrantz, 2001). These effects in blindsight patients have been termed affective blindsight. Functional MRI studies showed that both viewing fearful faces and the redundant target effect of fear induced amygdala activation (de Gelder, Morris, \& Dolan, 2005; Pegna et al., 2005).

These studies of lesioned patients indicate that the emotional information was relayed to the amygdala through a non-cortical pathway bypassing V1 (M. Tamietto \& de Gelder, 2010). Apart from the primary pathway that relays information from the lateral geniculate nucleus (LGN) to V1, two secondary subcortical pathways are proposed to be still functional in blindsight patients: the retino-tectal pathway is mediated through the superior colliculus (SC), the pulvinar, to the extrastriate cortex; the geniculo-extrastriate pathway is mediate through the koniocellular layers of LGN to the extrastriate cortex (for a schematic representation see Danckert \& Rossetti, 2005). In both the blindsight patient G.Y. and healthy controls, a DTI study showed fiber tracts from the amygdala to both the SC and the pulvinar (M. Tamietto et al., 2012). In healthy participants, the involvement of the retinotectal pathway in processing fearful faces was observed when they viewed low spatial frequency faces while performing a gender discrimination task, together with amygdala activation (Vuilleumier, Armony, Driver, \& Dolan, 2003). A follow-up study with intracranial electrophysiological recordings in epilepsy patients showed fast lateral amygdala responses (74 ms after stimulus onset) for both broadband and low spatial frequency fearful faces, while the fusiform gyrus did not show such fast responses (Mendez-Bertolo et al., 2016). These studies all point to the involvement of fast but coarse routes in (facial) emotion processing outside the ventral stream, and implicate the importance and necessity of keeping such fast emotional processing mechanisms through evolution in the human brain. 


\section{Chapter 1}

For healthy participants, the direct causal evidence for non-conscious processing came from TMS studies that disrupt activity in target brain areas. In one study, the hMT+ and V1 were both stimulated to induce overlapping moving and stationary phosphenes. When the subthreshold stimulation of V1 was applied 5 to $45 \mathrm{~ms}$ after the supra-threshold hMT+ stimulation, the participants reported decreased perception of moving phosphenes (PascualLeone \& Walsh, 2001), which indicated that a recurrent activity in the V1 (a feedback activity from other areas) is important for visual awareness (Lamme, 2006). Stimulating the V1 of healthy participants, blindsight- and affective blindsight-like behavior performances could be induced (Boyer, Harrison, \& Ro, 2005; Jolij \& Lamme, 2005). Other more indirect methods to study non-conscious processing includes masking, binocular rivalry and continuous flash suppression (CFS). For masking, electrophysiological recordings in the monkey V1 showed that forward and backward masking disrupted the activity respectively corresponding to the onset and offset of the stimulus (Macknik \& Livingstone, 1998); electroencephalogram (EEG) of human participants showed that, a component after $110 \mathrm{~ms}$ possibly corresponding to the reentrance processing in V1 was absent when the stimulus was masked and thus unseen (Fahrenfort, Scholte, \& Lamme, 2007). For binocular rivalry, an interocular competition is thought to be going on in either V1 or LGN, which disrupts the visibility of one of two dichoptically presented stimuli (Tong, Meng, \& Blake, 2006). The CFS is a more recently developed method than masking and binocular rivalry, where a low-contrast target stimulus presented in one eye is rendered invisible by a high-contrast (and colorful) dynamic noise pattern presented in the other eye, rendering stronger and more stable suppression than both masking and binocular rivalry (Tsuchiya \& Koch, 2005; Tsuchiya, Koch, Gilroy, \& Blake, 2006). Although the neural mechanisms of CFS is not fully understood yet, it is free of the drawback of masking, which renders stimuli "subconscious" instead of "non-conscious" (Kouider \& Dehaene, 2007), and free of the drawback of binocular rivalry with uncontrollable percept dominance. CFS has been increasingly used for the study of nonconscious processing, with three variant paradigms, including priming which measures the effect of the invisible prime on the visible target, breaking from suppression which measures the suppression time for stimuli, and stimuli being fully suppressed during neural response measurements (for reviews see Stein, Hebart, \& Sterzer, 2011; Yang, Brascamp, Kang, \& Blake, 2014). With non-emotional stimuli e.g. object categories, fMRI studies found that the ventral activity fluctuated with the subjective percept of the faces and tools, while the dorsal activity was less so (Fang \& He, 2005; but see Hesselmann, Hebart, \& Malach, 2011; Hesselmann \& Malach, 2011; Ludwig, Kathmann, Sterzer, \& Hesselmann, 2015).

For non-conscious/subconscious emotional face perception, these behavioral methods showed that the emotional faces were perceived with an advantage compared to nonemotional faces, showing longer dominance in binocular rivalry (e.g. Alpers \& Gerdes, 2007), and breaking from suppression faster (e.g. Yang, Zald, \& Blake, 2007). Functional MRI studies with unseen fearful faces showed that the activity of the ventral face-sensitive areas (FFA) co-varied with the subjective percept, showing less activity when the faces were unseen, but the amygdala activity was comparable between seen and unseen fearful faces (Jiang \& He, 2006; Tong, Nakayama, Vaughan, \& Kanwisher, 1998; Whalen et al., 1998; Williams, Morris, McGlone, Abbott, \& Mattingley, 2004). However, subsequent studies of both masking and CFS also indicated that the advantageous processing and amygdala activation for fearful 
faces may be related to the eye regions of the facial stimuli (Gray, Adams, Hedger, Newton, \& Garner, 2013; Whalen et al., 2004; Yang et al., 2007).

\section{The human bodies, a unique object category}

The human body is an object category conveying information such as identity, gender, and emotion, similar to faces. As a category of high behaviorally relevance, the processing of body forms has been studied with similar behavioral and neural methods to faces. Body- and body parts-specific areas have been found in the fusiform gyrus between face sensitive clusters, termed fusiform body area (FBA), and in the lateral occipital cortex, close to hMT+, termed extrastriate body area (EBA) (Downing, Jiang, Shuman, \& Kanwisher, 2001; Peelen \& Downing, 2005; Schwarzlose, Baker, \& Kanwisher, 2005; for a review see Peelen \& Downing, 2007. Also see Figure 1 and 4). For EBA, a study showed that the activity was modulated not only by passive perception of bodies, but also by participant's own unseen hand- and footpointing movements (Astafiev, Stanley, Shulman, \& Corbetta, 2004). Another study with higher $\mathrm{fMRI}$ resolution ( $1.5 \mathrm{~mm}$ isotropic) showed that the EBA was not one single cluster, but were three clusters surrounding the hMT+ (Weiner \& Grill-Spector, 2011). In addition to the body-specific EBA and FBA, the PSTS is activated by both bodies and faces, and is also activated by body motion (Kontaris, Wiggett, \& Downing, 2009; Pinsk et al., 2009; Vangeneugden, Peelen, Tadin, \& Battelli, 2014). Body-specific patches were also found in the monkey STS, partially overlapping with face-selective patches (de Gelder \& Partan, 2009; Popivanov, Jastorff, Vanduffel, \& Vogels, 2012, 2014). These patches had single-cell responses to local fragments of the bodies, which were frequently but not exclusively present with body stimuli (Popivanov, Schyns, \& Vogels, 2016).

An inversion effect has been found for bodies with behavioral and EEG studies, where the RT was longer for inverted bodies in a delayed match to sample task (Reed, Stone, Bozova, \& Tanaka, 2003); and the N170 component of event-related potentials (ERP) for both inverted faces and bodies were similarly delayed but enhanced (Stekelenburg \& de Gelder, 2004). One fMRI study suggested the importance of heads processed in ventral face-specific areas, because they found an $\mathrm{FMRI}$-adaptation effect for whole- comparing to headless- bodies in FFA and OFA, instead of in EBA and FBA (Brandman \& Yovel, 2010). However a later CFS behavioral study showed that the inverted bodies were suppressed longer than upright ones, with inversion effects equally strong for whole- and headless-bodies (Stein, Sterzer, \& Peelen, 2012).

In addition to the information related to visual forms and recognition, human bodies also convey a much wider range of daily-life actions than faces. Observing bodies activates the dorsal stream much more than faces, showing activation in the action observation network, including important nodes such as mIPS, aIPS, and PMv (compare Figure 4 to Figure 2). Studies indicated that the role of PMv and EBA in bodily action perception were different. A TMS study found that, in a delayed match-to-sample task where two upper/lower limb actions performed by two actors were shown to the participants, stimulating EBA selectively slowed the RT for form discrimination, while stimulating PMv selectively slowed the RT for action discrimination (Urgesi, Candidi, lonta, \& Aglioti, 2007). Another fMRI experiment found that, comparing to ordinary actions, when participants observed actions performed in 


\section{Chapter 1}

unusual means, and actions with unusual intentions, the inferior frontal sulcus (close to PMv) was activated more by unusual intentions, while the area close to EBA was activated more by unusual means (de Lange, Spronk, Willems, Toni, \& Bekkering, 2008).
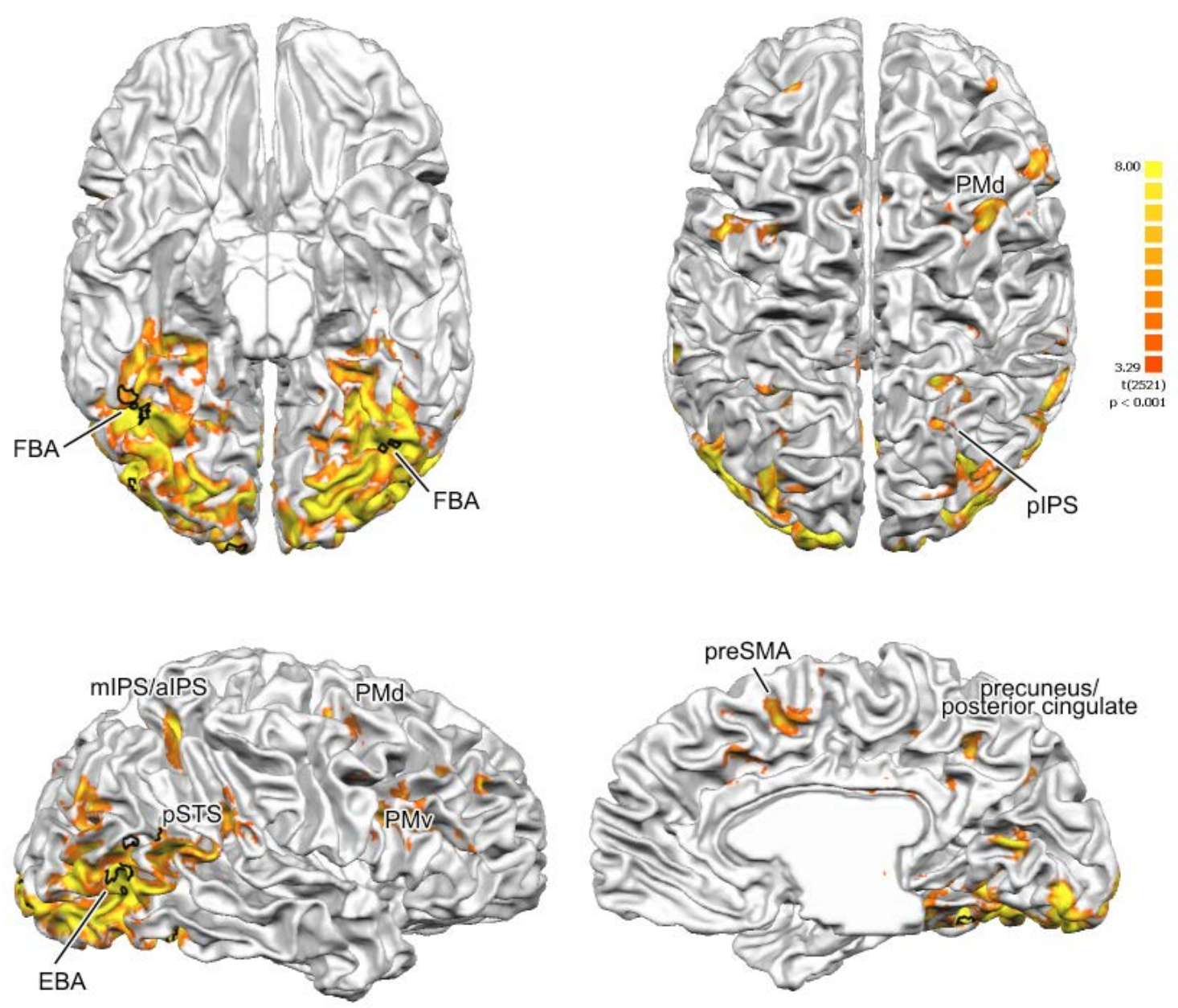

Figure 4. The cortical areas activated by perceiving whole-body images of emotional and nonemotional actions. The contrast maps were from the data of the same participant in Figure 1 (data of main experiment from Chapter 6, 7T, resolution $1.2 \mathrm{~mm}$ isotropic, smoothed with $3 \mathrm{~mm}$ FWHM, 10 action categories > baseline, $p=.001$ uncorrected), mapped on the cortical surface of the same participant. The body-specific areas shown in Figure 1 are traced out with black lines. Abbreviations: FBA: fusiform body area; EBA: extrastriate body area; PMd/PMv: dorsal/ventral premotor cortices; IPS: intraparietal sulcus; PSTS: posterior superior temporal sulcus; preSMA: pre-supplementary motor area.

Emotional information could be conveyed with bodies efficiently, either depicted with static images, or with dynamic video clips, or even with static or dynamic point-light displays alone (Atkinson, Dittrich, Gemmell, \& Young, 2004). The processing of bodily emotional information is fast in healthy participants. In a magnetoencephalography (MEG) study, the fearful bodies could be differentiated from neutral ones as early as $80 \mathrm{~ms}$ in parietal areas 


\section{Introduction}

(Meeren, Hadjikhani, Ahlfors, Hamalainen, \& de Gelder, 2016). The emotional bodies often provide contextual information for processing other modalities such as faces, voices, and scenes, and interfere with them (Meeren, van Heijnsbergen, \& de Gelder, 2005; J. Van den Stock \& de Gelder, 2012; J. Van den Stock \& de Gelder, 2014); but the perception of emotional bodies are also influenced by these kinds of contextual information (Kret \& de Gelder, 2010; Stienen, Tanaka, \& de Gelder, 2011).

Functional MRI studies showed that, the emotional bodies activated the amygdala (Hadjikhani \& de Gelder, 2003; Kret, Pichon, Grezes, \& de Gelder, 2011; Sinke, Sorger, Goebel, \& de Gelder, 2010; van de Riet, Grezes, \& de Gelder, 2009), modulated body-specific areas i.e. EBA, FBA, and pSTS (Atkinson, Vuong, \& Smithson, 2012; de Gelder, Snyder, Greve, Gerard, \& Hadjikhani, 2004; Grezes, Adenis, Pouga, \& Armony, 2013; Grezes, Pichon, \& de Gelder, 2007; Hadjikhani \& de Gelder, 2003; Kret et al., 2011; Peelen, Atkinson, Andersson, \& Vuilleumier, 2007; Peelen et al., 2010; Pichon, de Gelder, \& Grezes, 2008, 2012), similar to the emotional faces recruiting the amygdala and the face-specific areas. When fearful/neutral faces or triangles were placed in threatening scenes, the EBA could also be activated (Sinke, Van den Stock, Goebel, \& de Gelder, 2012). In addition, the emotional bodies also recruited action processing areas including PMv, PMd, and emotion-related areas including TPJ, temporal pole, orbitofrontal cortex (de Gelder et al., 2004; Grezes et al., 2013; Grezes et al., 2007; Kret et al., 2011; Pichon et al., 2008; Pichon, de Gelder, \& Grezes, 2009; Pichon et al., 2012).

TMS studies had examined the roles of PMv, EBA and parietal regions (IPL, aIPS) in bodily emotion processing, focusing more on the body-specificity and action-related aspects. Under binocular rivalry presentations of faces or bodies together with houses, stimulating pSTS decreased the dominance of fearful faces versus houses, but enhanced the dominance for fearful bodies (M Candidi, Aglioti, \& de Gelder, 2015). In a body posture discrimination task, stimulating pSTS enhanced the detection of posture changes in angry bodies but not in neutral bodies; however stimulating EBA and PMv did not show such effect dissociating between angry and neutral postures (M. Candidi, Stienen, Aglioti, \& de Gelder, 2011). In another similar task with fearful postures, stimulating IPL enhanced the processing of fearful bodies comparing to stimulating EBA (Engelen, de Graaf, Sack, \& de Gelder, 2015). A more recent study with happy and fearful point-light displays showed that, adapting to the emotion of the bodies would slow down the RT for targets with congruent emotion after the adaptation period, but this effect was abolished and even reversed specifically for fearful bodies, when stimulating alPS but not when stimulating pSTS (Mazzoni, Jacobs, Venuti, Silvanto, \& Cattaneo, 2017). These studies highlighted the importance of dorsal actionrelated areas in bodily emotion processing.

With the roles of bodies both conveying form and social information, and recruiting processing mechanisms in the dorsal stream, the study of emotional body perception provides a unique window for understanding the non-conscious/subconscious processes in the human brain. In patients with brain lesions, the emotional bodies showed attentionalgrabbing and privileged non-conscious processing similar to faces. In neglect patients, fearful bodies presented in the neglected visual field were less extinguished when comparing to neutral and happy bodies, showing an automatic attention-grabbing effect (M. Tamietto, 


\section{Chapter 1}

Geminiani, Genero, \& de Gelder, 2007). A subsequent fMRI study of a neglect patient showed left amygdala and EBA activation for both visible and extinguished fearful bodies, and the pupil dilation was bigger for extinguished than consciously perceived fearful bodies (M. Tamietto et al., 2015). For blindsight patients, viewing fearful and happy faces and bodies presented in the blind visual field induced fast reactions of the pupil and facial muscles (Marco Tamietto et al., 2009). For the patient G.Y., viewing unseen still and dynamic emotional bodies (happy, anger) recruited the retino-tectal pathway, inducing activation in the pulvinar and SC (de Gelder \& Hadjikhani, 2006; J. Van den Stock et al., 2011). For the patient T.N. with bilateral V1 lesions, passively viewing neutral stimuli of faces and bodies activated the amygdala and STS, and body stimuli additionally activated EBA, insula, orbitofrontal cortex, and the cerebellum (J. Van den Stock et al., 2014). In healthy participants, the subconscious processing of emotional bodies has been mainly studied with masking (Hortensius, van Honk, de Gelder, \& Terburg, 2014; Stienen \& de Gelder, 2011a; Stienen et al., 2011) and binocular rivalry (Stienen \& de Gelder, 2011b), which did not yet provide strictly non-conscious evidence.

\section{Outline of the thesis}

How are emotions expressed by bodily actions processed in the brain of healthy participants, both with and without visual awareness? In this thesis, we examined this question in three dimensions: the body, the emotion, and the visual awareness, using both behavioral (Chapter 2-4) and fMRI (Chapter 5-7) methods. For the body dimension, in Chapter 2 and Chapter 7, we compared the processing of body stimuli to that of the face stimuli. For the emotion dimension, we compared the different emotions expressed by bodily actions among each other in Chapter 2, 3, 7, and to non-emotional (instrumental) actions in Chapter 6 with 7T functional MRI. For the visual awareness dimension, in Chapter 2, 3, 5 we studied the body processing when the stimulus images were rendered invisible with CFS; in Chapter 4 we studied the temporal property of CFS itself: the flash frequencies of the masks; and in Chapter 6-7 we studied the processing under normal visible conditions.

We give a short description of each individual chapter below:

In Chapter 2, we measured the suppression time under the CFS paradigm, compared whether the non-conscious perception of the faces and bodies under CFS were similar, and whether the different emotions expressed with faces and bodies were processed similarly under such conditions. We found that the suppression time for the face and body stimuli in general differed, and the suppression time for different emotions also differed between the face and body stimuli. This suggests that the faces and bodies were processed in different ways in the brain, which is also the case for the same emotion expressed with either the face or the body.

In Chapter 3, to see if a link could be established between CFS and previous studies of both lesioned patient and healthy participants with backward masking, we further applied the CFS paradigm with face and body stimuli in another 3 behavioral experiments: affective priming, 
stimulus detection with confidence ratings, and bilateral presentation of emotional bodies with the redundant target paradigm. We found that CFS in general induced very strong suppression in all 3 experiments, the emotional information of the stimuli was hard to induce a behavioral dissociation between emotions, and the level of the stimuli being processed did not allow a redundant target effect to appear. The results of CFS were different from results of blindsight or neglect patients, but bore resemblance to that of hemianopia patients without blindsight. These results indicate that the unconscious processing is not a single phenomenon, and the ones induced in both lesioned patients and by CFS in healthy participants involved different mechanisms.

In Chapter 4, we examined the influence of the CFS masks 9 on stimulus visibility at 9 flash frequencies: the fundamental frequencies of 3,4 and $5 \mathrm{~Hz}$, and their $2^{\text {nd }}$ and $3^{\text {rd }}$ harmonics. This included the routinely used frequency of $10 \mathrm{~Hz}$ in most of the CFS studies. We found that the suppression strength was stronger at 4,6 and $8 \mathrm{~Hz}$ comparing to $10 \mathrm{~Hz}$, which hints that the CFS masks may be interacting with the attentional mechanisms that were found sampling the visual scene at $\sim 7 \mathrm{~Hz}$. We also found considerable inter-individual variability across frequencies, and a tendency that participants saw more stimuli during the course of the CFS experiments. All these findings impact the generalizability of CFS results, and these factors need to be taken care of in future CFS studies.

In Chapter 5, we examined the neural correlates of non-conscious bodily posture perception in the ventral and dorsal streams, and whether the visibility was interacting with the processing of forms, specifically the orientation of the bodies. We presented upright and inverted fearful bodies (the same ones used in Chapter 2) under CFS, rendering them either visible or invisible, and acquired functional data under 3T MRI. We saw a difference of activity between the ventral and dorsal streams. We found that the activity in the bodysensitive ventral areas (EBA, FBA) closely associated with the subjective percept of visibility. However the activity in the dorsal stream along the IPS (posterior, middle, anterior IPS) was significantly less linked to the subjective percept, especially in the posterior part of the IPS, showing an interaction of orientation and visibility. The results showed different involvements of closely localized dorsal areas, and hints the cooperative involvements of both the ventral and dorsal areas in processing body postures.

In Chapter 6, we further looked into the processing of whole-body postures in increased detail. We used a big stimulus set (80 stimuli in total) containing 10 different categories of daily actions, which varied in the implied motion, valence, emotional contents, and actor identities. With this stimulus set covering much wider categories than usually examined in the literature, we further recorded the brain activity in high resolution under 7T MRI, and applied the multivariate analysis (RSA) to examine the relationships of neural representation between stimuli pairs. The 10 -action-category structure could be found in areas related to body form processing, action processing, emotion processing, and attention processing. Importantly, we also found this 10-category structure in frontal, medial, subcortical and cerebellar areas, some of which did not show consistent univariate activation for any of the categories. The results suggest that the network involved in the action processing was much wider than previous thought. 


\section{Chapter 1}

In Chapter 7, we presented a short immersive virtual reality scenario of home violence to male participants between two scanning sessions, and examined its influence on the perception of emotional faces and bodies, displayed by male and female identities, morphed between fearful and happy expressions. We specifically examined whether the participants were actively empathizing towards the emotional stimuli after the VR experience, by looking into the activity in emotion-related (amygdala), empathy-related (default-mode network areas), and fusiform ROls across 4 runs of the main task. We found a decrease of activity in the temporo-parietal junction and the posterior cingulate cortex specifically for fearful faces, but not for other conditions. The amygdala and fusiform activity suggested that this decrease in DMN areas was not likely to be enhanced processing towards the stimuli per se, but was rather likely to be associated with internal reflections.

In Chapter 8, we offered a general discussion. We considered 1) the methodologies used in our experiments, and their implication in interpreting the results for understanding body and emotion perception; 2 ) the processing differences of bodies to faces, and the involvement of amygdala in emotion processing; 3 ) the insights we obtained from our experiments, for the mechanisms of CFS, and for the nuanced non-conscious processing in general; 4) the challenges of group inference with 7T data. 


\section{References}

Allison, T., Puce, A., \& McCarthy, G. (2000). Social perception from visual cues: role of the STS region. Trends Cogn Sci, 4(7), 267-278.

Almeida, J., Mahon, B. Z., Nakayama, K., \& Caramazza, A. (2008). Unconscious processing dissociates along categorical lines. Proc Natl Acad Sci U S A, 105(39), 15214-15218. doi: 10.1073/pnas.0805867105

Alpers, G. W., \& Gerdes, A. B. (2007). Here is looking at you: emotional faces predominate in binocular rivalry. Emotion, 7(3), 495-506. doi: 10.1037/1528-3542.7.3.495

Andersen, R. A., Andersen, K. N., Hwang, E. J., \& Hauschild, M. (2014). Optic ataxia: from Balint's syndrome to the parietal reach region. Neuron, 81(5), 967-983. doi: 10.1016/j.neuron.2014.02.025

Astafiev, S. V., Stanley, C. M., Shulman, G. L., \& Corbetta, M. (2004). Extrastriate body area in human occipital cortex responds to the performance of motor actions. Nat Neurosci, 7(5), 542-548. doi: $10.1038 / \mathrm{nn} 1241$

Atkinson, A. P., Dittrich, W. H., Gemmell, A. J., \& Young, A. W. (2004). Emotion perception from dynamic and static body expressions in point-light and full-light displays. Perception, 33(6), 717-746. doi: 10.1068/p5096

Atkinson, A. P., Vuong, Q. C., \& Smithson, H. E. (2012). Modulation of the face- and body-selective visual regions by the motion and emotion of point-light face and body stimuli. Neuroimage, 59(2), 1700-1712. doi: 10.1016/j.neuroimage.2011.08.073

Baker, C. I., Liu, J., Wald, L. L., Kwong, K. K., Benner, T., \& Kanwisher, N. (2007). Visual word processing and experiential origins of functional selectivity in human extrastriate cortex. Proc Natl Acad Sci U S A, 104(21), 9087-9092. doi: 10.1073/pnas.0703300104

Barendregt, M., Harvey, B. M., Rokers, B., \& Dumoulin, S. O. (2015). Transformation from a Retinal to a Cyclopean Representation in Human Visual Cortex. Curr Biol, 25(15), 1982-1987. doi: 10.1016/j.cub.2015.06.003

Boyer, J. L., Harrison, S., \& Ro, T. (2005). Unconscious processing of orientation and color without primary visual cortex. Proc Natl Acad Sci U S A, 102(46), 16875-16879. doi: 10.1073/pnas.0505332102

Brandman, T., \& Yovel, G. (2010). The body inversion effect is mediated by face-selective, not bodyselective, mechanisms. J Neurosci, 30(31), 10534-10540. doi: 10.1523/JNEUROSCI.091110.2010

Bridge, H., Thomas, O., Jbabdi, S., \& Cowey, A. (2008). Changes in connectivity after visual cortical brain damage underlie altered visual function. Brain, 131(Pt 6), 1433-1444. doi: 10.1093/brain/awn063

Buckner, R. L., Andrews-Hanna, J. R., \& Schacter, D. L. (2008). The brain's default network: anatomy, function, and relevance to disease. Ann N Y Acad Sci, 1124, 1-38. doi: 10.1196/annals.1440.011

Buneo, C. A., \& Andersen, R. A. (2006). The posterior parietal cortex: sensorimotor interface for the planning and online control of visually guided movements. Neuropsychologia, 44(13), 25942606. doi: 10.1016/j.neuropsychologia.2005.10.011

Bzdok, D., Schilbach, L., Vogeley, K., Schneider, K., Laird, A. R., Langner, R., \& Eickhoff, S. B. (2012). Parsing the neural correlates of moral cognition: ALE meta-analysis on morality, theory of mind, and empathy. Brain Struct Funct, 217(4), 783-796. doi: 10.1007/s00429-012-0380-y

Candidi, M., Aglioti, S., \& de Gelder, B. (2015). Virtual lesion of right posterior superior temporal sulcus modulates conscious visual perception of fearful expressions in faces and bodies. Cortex. 


\section{Chapter 1}

Candidi, M., Stienen, B. M., Aglioti, S. M., \& de Gelder, B. (2011). Event-related repetitive transcranial magnetic stimulation of posterior superior temporal sulcus improves the detection of threatening postural changes in human bodies. J Neurosci, 31(48), 17547-17554. doi: 10.1523/JNEUROSCI.0697-11.2011

Caspers, S., Zilles, K., Laird, A. R., \& Eickhoff, S. B. (2010). ALE meta-analysis of action observation and imitation in the human brain. Neuroimage, 50(3), 1148-1167. doi: 10.1016/j.neuroimage.2009.12.112

Cheng, K. (2016). Exploration of human visual cortex using high spatial resolution functional magnetic resonance imaging. Neuroimage. doi: 10.1016/j.neuroimage.2016.11.018

Chikazoe, J., Lee, D. H., Kriegeskorte, N., \& Anderson, A. K. (2014). Population coding of affect across stimuli, modalities and individuals. Nat Neurosci, 17(8), 1114-1122. doi: 10.1038/nn.3749

Clark, V. P., Keil, K., Maisog, J. M., Courtney, S., Ungerleider, L. G., \& Haxby, J. V. (1996). Functional magnetic resonance imaging of human visual cortex during face matching: a comparison with positron emission tomography. Neuroimage, 4(1), 1-15. doi: 10.1006/nimg.1996.0025

Cohen, L., Dehaene, S., Naccache, L., Lehericy, S., Dehaene-Lambertz, G., Henaff, M. A., \& Michel, F. (2000). The visual word form area: spatial and temporal characterization of an initial stage of reading in normal subjects and posterior split-brain patients. Brain, 123 ( Pt 2), 291-307.

Corbetta, M., Patel, G., \& Shulman, G. L. (2008). The reorienting system of the human brain: from environment to theory of mind. Neuron, 58(3), 306-324. doi: 10.1016/j.neuron.2008.04.017

Corbetta, M., \& Shulman, G. L. (2011). Spatial neglect and attention networks. Annu Rev Neurosci, 34, 569-599. doi: 10.1146/annurev-neuro-061010-113731

Cross, E. S., Kraemer, D. J., Hamilton, A. F., Kelley, W. M., \& Grafton, S. T. (2009). Sensitivity of the action observation network to physical and observational learning. Cereb Cortex, 19(2), 315326. doi: 10.1093/cercor/bhn083

Culham, J. C., Cavina-Pratesi, C., \& Singhal, A. (2006). The role of parietal cortex in visuomotor control: what have we learned from neuroimaging? Neuropsychologia, 44(13), 2668-2684. doi: 10.1016/j.neuropsychologia.2005.11.003

Culham, J. C., \& Valyear, K. F. (2006). Human parietal cortex in action. Curr Opin Neurobiol, 16(2), 205212. doi: 10.1016/j.conb.2006.03.005

Danckert, J., \& Rossetti, Y. (2005). Blindsight in action: what can the different sub-types of blindsight tell us about the control of visually guided actions? Neurosci Biobehav Rev, 29(7), 1035-1046. doi: 10.1016/j.neubiorev.2005.02.001

de Gelder, B., \& Hadjikhani, N. (2006). Non-conscious recognition of emotional body language. Neuroreport, 17(6), 583-586.

de Gelder, B., Morris, J. S., \& Dolan, R. J. (2005). Unconscious fear influences emotional awareness of faces and voices. Proc Natl Acad Sci U S A, 102(51), 18682-18687. doi: 10.1073/pnas.0509179102

de Gelder, B., \& Partan, S. (2009). The neural basis of perceiving emotional bodily expressions in monkeys. Neuroreport, 20(7), 642-646. doi: 10.1097/WNR.0b013e32832a1e56

de Gelder, B., Pourtois, G., van Raamsdonk, M., Vroomen, J., \& Weiskrantz, L. (2001). Unseen stimuli modulate conscious visual experience: evidence from inter-hemispheric summation. Neuroreport, 12(2), 385-391.

de Gelder, B., \& Rouw, R. (2000). Configural face processes in acquired and developmental prosopagnosia: evidence for two separate face systems? Neuroreport, 11(14), 3145-3150.

de Gelder, B., Snyder, J., Greve, D., Gerard, G., \& Hadjikhani, N. (2004). Fear fosters flight: a mechanism for fear contagion when perceiving emotion expressed by a whole body. Proc Natl Acad Sci U S A, 101(47), 16701-16706. doi: 10.1073/pnas.0407042101 
de Gelder, B., Tamietto, M., Van Boxtel, G., Goebel, R., Sahraie, A., Van den Stock, J., . . Pegna, A. (2008). Intact navigation skills after bilateral loss of striate cortex. Curr Biol, 18(24), R11281129. doi: 10.1016/j.cub.2008.11.002

de Gelder, B., Vroomen, J., Pourtois, G., \& Weiskrantz, L. (1999). Non-conscious recognition of affect in the absence of striate cortex. Neuroreport, 10(18), 3759-3763.

de Lange, F. P., Spronk, M., Willems, R. M., Toni, I., \& Bekkering, H. (2008). Complementary systems for understanding action intentions. Curr Biol, 18(6), 454-457. doi: 10.1016/j.cub.2008.02.057

De Martino, F., Yacoub, E., Kemper, V., Moerel, M., Uludag, K., De Weerd, P., . . Formisano, E. (2017). The impact of ultra-high field MRI on cognitive and computational neuroimaging. Neuroimage. doi: 10.1016/j.neuroimage.2017.03.060

Decety, J., \& Lamm, C. (2007). The role of the right temporoparietal junction in social interaction: how low-level computational processes contribute to meta-cognition. Neuroscientist, 13(6), 580593. doi: $10.1177 / 1073858407304654$

Demos, K. E., Kelley, W. M., Ryan, S. L., Davis, F. C., \& Whalen, P. J. (2008). Human amygdala sensitivity to the pupil size of others. Cereb Cortex, 18(12), 2729-2734. doi: 10.1093/cercor/bhn034

di Pellegrino, G., \& Ladavas, E. (2015). Peripersonal space in the brain. Neuropsychologia, 66, 126-133. doi: 10.1016/j.neuropsychologia.2014.11.011

Downing, P. E., Jiang, Y., Shuman, M., \& Kanwisher, N. (2001). A cortical area selective for visual processing of the human body. Science, 293(5539), 2470-2473. doi: 10.1126/science.1063414

Dricu, M., \& Fruhholz, S. (2016). Perceiving emotional expressions in others: Activation likelihood estimation meta-analyses of explicit evaluation, passive perception and incidental perception of emotions. Neurosci Biobehav Rev, 71, 810-828. doi: 10.1016/j.neubiorev.2016.10.020

Driver, J., \& Mattingley, J. B. (1998). Parietal neglect and visual awareness. Nat Neurosci, 1(1), 17-22. doi: 10.1038/217

Driver, J., \& Vuilleumier, P. (2001). Perceptual awareness and its loss in unilateral neglect and extinction. Cognition, 79(1-2), 39-88.

Dumoulin, S. O., Fracasso, A., van der Zwaag, W., Siero, J. C. W., \& Petridou, N. (2017). Ultra-high field MRI: Advancing systems neuroscience towards mesoscopic human brain function. Neuroimage. doi: 10.1016/j.neuroimage.2017.01.028

Engelen, T., de Graaf, T. A., Sack, A. T., \& de Gelder, B. (2015). A causal role for inferior parietal lobule in emotion body perception. Cortex, 73, 195-202. doi: 10.1016/j.cortex.2015.08.013

Epstein, R., \& Kanwisher, N. (1998). A cortical representation of the local visual environment. Nature, 392(6676), 598-601. doi: 10.1038/33402

Fahrenfort, J. J., Scholte, H. S., \& Lamme, V. A. (2007). Masking disrupts reentrant processing in human visual cortex. J Cogn Neurosci, 19(9), 1488-1497. doi: 10.1162/jocn.2007.19.9.1488

Fang, F., \& He, S. (2005). Cortical responses to invisible objects in the human dorsal and ventral pathways. Nat Neurosci, 8(10), 1380-1385. doi: 10.1038/nn1537

Farah, M. J., Tanaka, J. W., \& Drain, H. M. (1995). What causes the face inversion effect? J Exp Psychol Hum Percept Perform, 21(3), 628-634.

Farah, M. J., Wilson, K. D., Drain, H. M., \& Tanaka, J. R. (1995). The inverted face inversion effect in prosopagnosia: evidence for mandatory, face-specific perceptual mechanisms. Vision Res, 35(14), 2089-2093.

Friston, K. J., Holmes, A. P., Worsley, K. J., Poline, J. P., Frith, C. D., \& Frackowiak, R. S. J. (1994). Statistical parametric maps in functional imaging: A general linear approach. Hum Brain Mapp, 2(4), 189-210. doi: 10.1002/hbm.460020402 


\section{Chapter 1}

Gauthier, I., Skudlarski, P., Gore, J. C., \& Anderson, A. W. (2000). Expertise for cars and birds recruits brain areas involved in face recognition. Nat Neurosci, 3(2), 191-197. doi: 10.1038/72140

Georgieva, S., Peeters, R., Kolster, H., Todd, J. T., \& Orban, G. A. (2009). The processing of threedimensional shape from disparity in the human brain. $J$ Neurosci, 29(3), 727-742. doi: 10.1523/jneurosci.4753-08.2009

Goodale, M. A., Jakobson, L. S., \& Keillor, J. M. (1994). Differences in the visual control of pantomimed and natural grasping movements. Neuropsychologia, 32(10), 1159-1178.

Grafton, S. T., \& Hamilton, A. F. (2007). Evidence for a distributed hierarchy of action representation in the brain. Hum Mov Sci, 26(4), 590-616. doi: 10.1016/j.humov.2007.05.009

Gray, K. L., Adams, W. J., Hedger, N., Newton, K. E., \& Garner, M. (2013). Faces and awareness: lowlevel, not emotional factors determine perceptual dominance. Emotion, 13(3), 537-544. doi: $10.1037 / \mathrm{a} 0031403$

Graziano, M. S., \& Cooke, D. F. (2006). Parieto-frontal interactions, personal space, and defensive behavior. Neuropsychologia, 44(6), 845-859. doi: 10.1016/j.neuropsychologia.2005.09.009

Grezes, J., Adenis, M. S., Pouga, L., \& Armony, J. L. (2013). Self-relevance modulates brain responses to angry body expressions. Cortex, 49(8), 2210-2220. doi: 10.1016/j.cortex.2012.08.025

Grezes, J., Pichon, S., \& de Gelder, B. (2007). Perceiving fear in dynamic body expressions. Neuroimage, 35(2), 959-967. doi: 10.1016/j.neuroimage.2006.11.030

Grill-Spector, K., \& Malach, R. (2004). The human visual cortex. Annu Rev Neurosci, 27, 649-677. doi: 10.1146/annurev.neuro.27.070203.144220

Grill-Spector, K., \& Weiner, K. S. (2014). The functional architecture of the ventral temporal cortex and its role in categorization. Nat Rev Neurosci, 15(8), 536-548. doi: 10.1038/nrn3747

Grossman, E. D., Battelli, L., \& Pascual-Leone, A. (2005). Repetitive TMS over posterior STS disrupts perception of biological motion. Vision Res, 45(22), 2847-2853. doi: 10.1016/j.visres.2005.05.027

Hadjikhani, N., \& de Gelder, B. (2003). Seeing Fearful Body Expressions Activates the Fusiform Cortex and Amygdala. Current Biology, 13(24), 2201-2205. doi: 10.1016/j.cub.2003.11.049

Haynes, J. D. (2015). A Primer on Pattern-Based Approaches to fMRI: Principles, Pitfalls, and Perspectives. Neuron, 87(2), 257-270. doi: 10.1016/j.neuron.2015.05.025

Hesselmann, G., Hebart, M., \& Malach, R. (2011). Differential BOLD activity associated with subjective and objective reports during "blindsight" in normal observers. J Neurosci, 31(36), 1293612944. doi: 10.1523/JNEUROSCI.1556-11.2011

Hesselmann, G., \& Malach, R. (2011). The link between fMRI-BOLD activation and perceptual awareness is "stream-invariant" in the human visual system. Cereb Cortex, 21(12), 2829-2837. doi: $10.1093 /$ cercor/bhr085

Hortensius, R., van Honk, J., de Gelder, B., \& Terburg, D. (2014). Trait dominance promotes reflexive staring at masked angry body postures. PLoS One, 9(12), e116232. doi: 10.1371/journal.pone.0116232

Igelstrom, K. M., \& Graziano, M. S. A. (2017). The inferior parietal lobule and temporoparietal junction: A network perspective. Neuropsychologia, 105, 70-83. doi: 10.1016/j.neuropsychologia.2017.01.001

Jakobson, L., Archibald, Y., Carey, D., \& Goodale, M. A. (1991). A kinematic analysis of reaching and grasping movements in a patient recovering from optic ataxia. Neuropsychologia, 29(8), 803809.

James, T. W., Culham, J., Humphrey, G. K., Milner, A. D., \& Goodale, M. A. (2003). Ventral occipital lesions impair object recognition but not object-directed grasping: an fMRI study. Brain, 126(Pt 11), 2463-2475. doi: 10.1093/brain/awg248

Janak, P. H., \& Tye, K. M. (2015). From circuits to behaviour in the amygdala. Nature, 517(7534), $284-$ 292. doi: $10.1038 /$ nature 14188 


\section{Introduction}

Jiang, Y., \& He, S. (2006). Cortical responses to invisible faces: dissociating subsystems for facialinformation processing. Curr Biol, 16(20), 2023-2029. doi: 10.1016/j.cub.2006.08.084

Johnson-Frey, S. H. (2004). The neural bases of complex tool use in humans. Trends Cogn Sci, 8(2), 7178.

Jolij, J., \& Lamme, V. A. (2005). Repression of unconscious information by conscious processing: evidence from affective blindsight induced by transcranial magnetic stimulation. Proc Natl Acad Sci U S A, 102(30), 10747-10751. doi: 10.1073/pnas.0500834102

Kanwisher, N., McDermott, J., \& Chun, M. M. (1997). The fusiform face area: a module in human extrastriate cortex specialized for face perception. J Neurosci, 17(11), 4302-4311.

Kiani, R., Esteky, H., Mirpour, K., \& Tanaka, K. (2007). Object category structure in response patterns of neuronal population in monkey inferior temporal cortex. J Neurophysiol, 97(6), 4296-4309. doi: $10.1152 /$ jn.00024.2007

Kober, H., Barrett, L. F., Joseph, J., Bliss-Moreau, E., Lindquist, K., \& Wager, T. D. (2008). Functional grouping and cortical-subcortical interactions in emotion: a meta-analysis of neuroimaging studies. Neuroimage, 42(2), 998-1031. doi: 10.1016/j.neuroimage.2008.03.059

Konkle, T., \& Oliva, A. (2012). A real-world size organization of object responses in occipitotemporal cortex. Neuron, 74(6), 1114-1124. doi: 10.1016/j.neuron.2012.04.036

Kontaris, I., Wiggett, A. J., \& Downing, P. E. (2009). Dissociation of extrastriate body and biologicalmotion selective areas by manipulation of visual-motor congruency. Neuropsychologia, 47(14), 3118-3124. doi: 10.1016/j.neuropsychologia.2009.07.012

Kouider, S., \& Dehaene, S. (2007). Levels of processing during non-conscious perception: a critical review of visual masking. Philos Trans R Soc Lond B Biol Sci, 362(1481), 857-875. doi: $10.1098 /$ rstb.2007.2093

Kragel, P. A., \& LaBar, K. S. (2015). Multivariate neural biomarkers of emotional states are categorically distinct. Soc Cogn Affect Neurosci, 10(11), 1437-1448. doi: 10.1093/scan/nsv032

Kret, M. E., \& de Gelder, B. (2010). Social context influences recognition of bodily expressions. Exp Brain Res, 203(1), 169-180. doi: 10.1007/s00221-010-2220-8

Kret, M. E., Pichon, S., Grezes, J., \& de Gelder, B. (2011). Similarities and differences in perceiving threat from dynamic faces and bodies. An fMRI study. Neuroimage, 54(2), 1755-1762. doi: 10.1016/j.neuroimage.2010.08.012

Kriegeskorte, N., Mur, M., Ruff, D. A., Kiani, R., Bodurka, J., Esteky, H., . . Bandettini, P. A. (2008). Matching categorical object representations in inferior temporal cortex of man and monkey. Neuron, 60(6), 1126-1141. doi: 10.1016/j.neuron.2008.10.043

Lamme, V. A. (2006). Towards a true neural stance on consciousness. Trends Cogn Sci, 10(11), 494-501. doi: 10.1016/j.tics.2006.09.001

LeDoux, J. (2003). The emotional brain, fear, and the amygdala. Cell Mol Neurobiol, 23(4-5), 727-738.

Ludwig, K., Kathmann, N., Sterzer, P., \& Hesselmann, G. (2015). Investigating category- and shapeselective neural processing in ventral and dorsal visual stream under interocular suppression. Hum Brain Mapp, 36(1), 137-149. doi: 10.1002/hbm.22618

Macknik, S. L., \& Livingstone, M. S. (1998). Neuronal correlates of visibility and invisibility in the primate visual system. Nat Neurosci, 1(2), 144-149. doi: 10.1038/393

Malach, R., Levy, I., \& Hasson, U. (2002). The topography of high-order human object areas. Trends Cogn Sci, 6(4), 176-184.

Malach, R., Reppas, J. B., Benson, R. R., Kwong, K. K., Jiang, H., Kennedy, W. A., . . Tootell, R. B. (1995). Object-related activity revealed by functional magnetic resonance imaging in human occipital cortex. Proc Natl Acad Sci U S A, 92(18), 8135-8139.

Mars, R. B., Neubert, F. X., Noonan, M. P., Sallet, J., Toni, I., \& Rushworth, M. F. (2012). On the relationship between the "default mode network" and the "social brain". Front Hum Neurosci, 6, 189. doi: 10.3389/fnhum.2012.00189 


\section{Chapter 1}

Mazzoni, N., Jacobs, C., Venuti, P., Silvanto, J., \& Cattaneo, L. (2017). State-Dependent TMS Reveals Representation of Affective Body Movements in the Anterior Intraparietal Cortex. J Neurosci, 37(30), 7231-7239. doi: 10.1523/jneurosci.0913-17.2017

McIntosh, R. D., McClements, K. I., Dijkerman, H. C., Birchall, D., \& Milner, A. D. (2004). Preserved obstacle avoidance during reaching in patients with left visual neglect. Neuropsychologia, 42(8), 1107-1117. doi: 10.1016/j.neuropsychologia.2003.11.023

McKeefry, D. J., \& Zeki, S. (1997). The position and topography of the human colour centre as revealed by functional magnetic resonance imaging. Brain, 120 ( Pt 12), 2229-2242.

Meeren, H. K., Hadjikhani, N., Ahlfors, S. P., Hamalainen, M. S., \& de Gelder, B. (2016). Early Preferential Responses to Fear Stimuli in Human Right Dorsal Visual Stream--A Meg Study. Sci Rep, 6, 24831. doi: 10.1038/srep24831

Meeren, H. K., van Heijnsbergen, C. C., \& de Gelder, B. (2005). Rapid perceptual integration of facial expression and emotional body language. Proc Natl Acad Sci U S A, 102(45), 16518-16523. doi: 10.1073/pnas.0507650102

Mendez-Bertolo, C., Moratti, S., Toledano, R., Lopez-Sosa, F., Martinez-Alvarez, R., Mah, Y. H., . . . Strange, B. A. (2016). A fast pathway for fear in human amygdala. Nat Neurosci, 19(8), 10411049. doi: $10.1038 / \mathrm{nn} .4324$

Milner, A. D., \& Goodale, M. A. (2006). The visual brain in action: Oxford University Press.

Milner, A. D., \& Goodale, M. A. (2008). Two visual systems re-viewed. Neuropsychologia, 46(3), 774785. doi: 10.1016/j.neuropsychologia.2007.10.005

Naselaris, T., Kay, K. N., Nishimoto, S., \& Gallant, J. L. (2011). Encoding and decoding in fMRI. Neuroimage, 56(2), 400-410. doi: 10.1016/j.neuroimage.2010.07.073

Nili, H., Wingfield, C., Walther, A., Su, L., Marslen-Wilson, W., \& Kriegeskorte, N. (2014). A toolbox for representational similarity analysis. PLoS Comput Biol, 10(4), e1003553. doi: 10.1371/journal.pcbi.1003553

Nowak, L. G., \& Bullier, J. (1997). The timing of information transfer in the visual system Extrastriate cortex in primates (pp. 205-241): Springer.

Ohman, A., \& Mineka, S. (2001). Fears, phobias, and preparedness: toward an evolved module of fear and fear learning. Psychol Rev, 108(3), 483-522.

Orban, G. A. (2011). The extraction of 3D shape in the visual system of human and nonhuman primates. Annu Rev Neurosci, 34, 361-388. doi: 10.1146/annurev-neuro-061010-113819

Orban, G. A. (2016). Functional definitions of parietal areas in human and non-human primates. Proc Biol Sci, 283(1828). doi: 10.1098/rspb.2016.0118

Pascual-Leone, A., \& Walsh, V. (2001). Fast backprojections from the motion to the primary visual area necessary for visual awareness. Science, 292(5516), 510-512. doi: 10.1126/science.1057099

Peelen, M. V., Atkinson, A. P., Andersson, F., \& Vuilleumier, P. (2007). Emotional modulation of bodyselective visual areas. Soc Cogn Affect Neurosci, 2(4), 274-283. doi: 10.1093/scan/nsm023

Peelen, M. V., Atkinson, A. P., \& Vuilleumier, P. (2010). Supramodal representations of perceived emotions in the human brain. J Neurosci, 30(30), 10127-10134. doi: 10.1523/JNEUROSCI.2161-10.2010

Peelen, M. V., \& Downing, P. E. (2005). Selectivity for the human body in the fusiform gyrus. J Neurophysiol, 93(1), 603-608. doi: 10.1152/jn.00513.2004

Peelen, M. V., \& Downing, P. E. (2007). The neural basis of visual body perception. Nat Rev Neurosci, 8(8), 636-648. doi: 10.1038/nrn2195

Pegna, A. J., Khateb, A., Lazeyras, F., \& Seghier, M. L. (2005). Discriminating emotional faces without primary visual cortices involves the right amygdala. Nat Neurosci, 8(1), 24-25. doi: $10.1038 / \mathrm{nn} 1364$ 


\section{Introduction}

Pessoa, L. (2010). Emotion and cognition and the amygdala: from "what is it?" to "what's to be done?". Neuropsychologia, 48(12), 3416-3429. doi: 10.1016/j.neuropsychologia.2010.06.038

Pichon, S., de Gelder, B., \& Grezes, J. (2008). Emotional modulation of visual and motor areas by dynamic body expressions of anger. Soc Neurosci, 3(3-4), 199-212. doi: 10.1080/17470910701394368

Pichon, S., de Gelder, B., \& Grezes, J. (2009). Two different faces of threat. Comparing the neural systems for recognizing fear and anger in dynamic body expressions. Neuroimage, 47(4), 1873-1883. doi: 10.1016/j.neuroimage.2009.03.084

Pichon, S., de Gelder, B., \& Grezes, J. (2012). Threat prompts defensive brain responses independently of attentional control. Cereb Cortex, 22(2), 274-285. doi: 10.1093/cercor/bhr060

Pinsk, M. A., Arcaro, M., Weiner, K. S., Kalkus, J. F., Inati, S. J., Gross, C. G., \& Kastner, S. (2009). Neural representations of faces and body parts in macaque and human cortex: a comparative FMRI study. J Neurophysiol, 101(5), 2581-2600. doi: 10.1152/jn.91198.2008

Popivanov, I. D., Jastorff, J., Vanduffel, W., \& Vogels, R. (2012). Stimulus representations in bodyselective regions of the macaque cortex assessed with event-related fMRI. Neuroimage, 63(2), 723-741. doi: 10.1016/j.neuroimage.2012.07.013

Popivanov, I. D., Jastorff, J., Vanduffel, W., \& Vogels, R. (2014). Heterogeneous single-unit selectivity in an fMRI-defined body-selective patch. J Neurosci, 34(1), 95-111. doi: 10.1523/jneurosci.2748-13.2014

Popivanov, I. D., Schyns, P. G., \& Vogels, R. (2016). Stimulus features coded by single neurons of a macaque body category selective patch. Proc Natl Acad Sci U S A, 113(17), E2450-2459. doi: 10.1073/pnas.1520371113

Reed, C. L., Stone, V. E., Bozova, S., \& Tanaka, J. (2003). The body-inversion effect. Psychol Sci, 14(4), 302-308.

Rizzolatti, G., \& Craighero, L. (2004). The mirror-neuron system. Annu Rev Neurosci, 27, 169-192. doi: 10.1146/annurev.neuro.27.070203.144230

Rizzolatti, G., \& Sinigaglia, C. (2010). The functional role of the parieto-frontal mirror circuit: interpretations and misinterpretations. Nature Reviews Neuroscience, 11(4), 264-274.

Saarimaki, H., Gotsopoulos, A., Jaaskelainen, I. P., Lampinen, J., Vuilleumier, P., Hari, R., . . . Nummenmaa, L. (2016). Discrete Neural Signatures of Basic Emotions. Cereb Cortex, 26(6), 2563-2573. doi: 10.1093/cercor/bhv086

Sakuraba, S., Sakai, S., Yamanaka, M., Yokosawa, K., \& Hirayama, K. (2012). Does the human dorsal stream really process a category for tools? J Neurosci, 32(11), 3949-3953. doi: 10.1523/JNEUROSCI.3973-11.2012

Sander, D., Grafman, J., \& Zalla, T. (2003). The human amygdala: an evolved system for relevance detection. Rev Neurosci, 14(4), 303-316.

Saxe, R., \& Kanwisher, N. (2003). People thinking about thinking people. The role of the temporoparietal junction in "theory of mind". Neuroimage, 19(4), 1835-1842.

Schluppeck, D., Sanchez-Panchuelo, R. M., \& Francis, S. T. (2017). Exploring structure and function of sensory cortex with 7T MRI. Neuroimage. doi: 10.1016/j.neuroimage.2017.01.081

Schurz, M., Tholen, M. G., Perner, J., Mars, R. B., \& Sallet, J. (2017). Specifying the brain anatomy underlying temporo-parietal junction activations for theory of mind: A review using probabilistic atlases from different imaging modalities. Hum Brain Mapp, 38(9), 4788-4805. doi: 10.1002/hbm.23675

Schwarzlose, R. F., Baker, C. I., \& Kanwisher, N. (2005). Separate face and body selectivity on the fusiform gyrus. J Neurosci, 25(47), 11055-11059. doi: 10.1523/jneurosci.2621-05.2005

Sergerie, K., Chochol, C., \& Armony, J. L. (2008). The role of the amygdala in emotional processing: a quantitative meta-analysis of functional neuroimaging studies. Neurosci Biobehav Rev, 32(4), 811-830. doi: 10.1016/j.neubiorev.2007.12.002 


\section{Chapter 1}

Sinke, C. B., Sorger, B., Goebel, R., \& de Gelder, B. (2010). Tease or threat? Judging social interactions from bodily expressions. Neuroimage, 49(2), 1717-1727. doi: 10.1016/j.neuroimage.2009.09.065

Sinke, C. B., Van den Stock, J., Goebel, R., \& de Gelder, B. (2012). The constructive nature of affective vision: seeing fearful scenes activates extrastriate body area. PLoS One, 7(6), e38118. doi: 10.1371/journal.pone.0038118

Skerry, A. E., \& Saxe, R. (2015). Neural representations of emotion are organized around abstract event features. Curr Biol, 25(15), 1945-1954. doi: 10.1016/j.cub.2015.06.009

Sladky, R., Baldinger, P., Kranz, G. S., Tröstl, J., Höflich, A., Lanzenberger, R., . . Windischberger, C. (2013). High-resolution functional MRI of the human amygdala at 7 T. Eur J Radiol, 82(5), 728-733. doi: 10.1016/j.ejrad.2011.09.025

Stein, T., Hebart, M. N., \& Sterzer, P. (2011). Breaking Continuous Flash Suppression: A New Measure of Unconscious Processing during Interocular Suppression? Front Hum Neurosci, 5, 167. doi: 10.3389/fnhum.2011.00167

Stein, T., Sterzer, P., \& Peelen, M. V. (2012). Privileged detection of conspecifics: evidence from inversion effects during continuous flash suppression. Cognition, 125(1), 64-79. doi: 10.1016/j.cognition.2012.06.005

Stekelenburg, J. J., \& de Gelder, B. (2004). The neural correlates of perceiving human bodies: an ERP study on the body-inversion effect. Neuroreport, 15(5), 777-780.

Stienen, B. M., \& de Gelder, B. (2011a). Fear detection and visual awareness in perceiving bodily expressions. Emotion, 11(5), 1182-1189. doi: 10.1037/a0024032

Stienen, B. M., \& de Gelder, B. (2011b). Fear modulates visual awareness similarly for facial and bodily expressions. Front Hum Neurosci, 5, 132. doi: 10.3389/fnhum.2011.00132

Stienen, B. M., Tanaka, A., \& de Gelder, B. (2011). Emotional voice and emotional body postures influence each other independently of visual awareness. PLoS One, 6(10), e25517. doi: 10.1371/journal.pone.0025517

Tamietto, M., Castelli, L., Vighetti, S., Perozzo, P., Geminiani, G., Weiskrantz, L., \& de Gelder, B. (2009). Unseen facial and bodily expressions trigger fast emotional reactions. Proceedings of the National Academy of Sciences, 106(42), 17661-17666.

Tamietto, M., Cauda, F., Celeghin, A., Diano, M., Costa, T., Cossa, F. M., . . . de Gelder, B. (2015). Once you feel it, you see it: Insula and sensory-motor contribution to visual awareness for fearful bodies in parietal neglect. Cortex, 62, 56-72. doi: 10.1016/j.cortex.2014.10.009

Tamietto, M., \& de Gelder, B. (2010). Neural bases of the non-conscious perception of emotional signals. Nat Rev Neurosci, 11(10), 697-709. doi: 10.1038/nrn2889

Tamietto, M., Geminiani, G., Genero, R., \& de Gelder, B. (2007). Seeing fearful body language overcomes attentional deficits in patients with neglect. J Cogn Neurosci, 19(3), 445-454.

Tamietto, M., Pullens, P., de Gelder, B., Weiskrantz, L., \& Goebel, R. (2012). Subcortical connections to human amygdala and changes following destruction of the visual cortex. Curr Biol, 22(15), 1449-1455. doi: 10.1016/j.cub.2012.06.006

Tong, F., Meng, M., \& Blake, R. (2006). Neural bases of binocular rivalry. Trends Cogn Sci, 10(11), $502-$ 511. doi: 10.1016/j.tics.2006.09.003

Tong, F., Nakayama, K., Vaughan, J. T., \& Kanwisher, N. (1998). Binocular rivalry and visual awareness in human extrastriate cortex. Neuron, 21(4), 753-759.

Tsao, D. Y., Freiwald, W. A., Knutsen, T. A., Mandeville, J. B., \& Tootell, R. B. (2003). Faces and objects in macaque cerebral cortex. Nat Neurosci, 6(9), 989-995. doi: 10.1038/nn1111

Tsao, D. Y., Freiwald, W. A., Tootell, R. B., \& Livingstone, M. S. (2006). A cortical region consisting entirely of face-selective cells. Science, 311(5761), 670-674. doi: 10.1126/science.1119983

Tsuchiya, N., \& Koch, C. (2005). Continuous flash suppression reduces negative afterimages. Nat Neurosci, 8(8), 1096-1101. doi: 10.1038/nn1500 


\section{Introduction}

Tsuchiya, N., Koch, C., Gilroy, L. A., \& Blake, R. (2006). Depth of interocular suppression associated with continuous flash suppression, flash suppression, and binocular rivalry. J Vis, 6(10), 10681078. doi: $10.1167 / 6.10 .6$

Ugurbil, K. (2014). Magnetic resonance imaging at ultrahigh fields. IEEE Trans Biomed Eng, 61(5), 1364-1379. doi: 10.1109/tbme.2014.2313619

Urgesi, C., Candidi, M., lonta, S., \& Aglioti, S. M. (2007). Representation of body identity and body actions in extrastriate body area and ventral premotor cortex. Nat Neurosci, 10(1), 30-31. doi: $10.1038 / \mathrm{nn} 1815$

van de Riet, W. A., Grezes, J., \& de Gelder, B. (2009). Specific and common brain regions involved in the perception of faces and bodies and the representation of their emotional expressions. Soc Neurosci, 4(2), 101-120. doi: 10.1080/17470910701865367

Van den Stock, J., \& de Gelder, B. (2012). Emotional information in body and background hampers recognition memory for faces. Neurobiol Learn Mem, 97(3), 321-325. doi: 10.1016/j.nlm.2012.01.007

Van den Stock, J., \& de Gelder, B. (2014). Face identity matching is influenced by emotions conveyed by face and body. Front Hum Neurosci, 8, 53. doi: 10.3389/fnhum.2014.00053

Van den Stock, J., Tamietto, M., Sorger, B., Pichon, S., Grézes, J., \& de Gelder, B. (2011). Corticosubcortical visual, somatosensory, and motor activations for perceiving dynamic whole-body emotional expressions with and without striate cortex (V1). Proceedings of the National Academy of Sciences, 108(39), 16188-16193.

Van den Stock, J., Tamietto, M., Zhan, M., Heinecke, A., Hervais-Adelman, A., Legrand, L. B., ... de Gelder, B. (2014). Neural correlates of body and face perception following bilateral destruction of the primary visual cortices. Front Behav Neurosci, 8, 30. doi: 10.3389/fnbeh.2014.00030

Vangeneugden, J., Peelen, M. V., Tadin, D., \& Battelli, L. (2014). Distinct neural mechanisms for body form and body motion discriminations. J Neurosci, 34(2), 574-585. doi: 10.1523/jneurosci.4032-13.2014

Vuilleumier, P., Armony, J. L., Clarke, K., Husain, M., Driver, J., \& Dolan, R. J. (2002). Neural response to emotional faces with and without awareness: event-related fMRI in a parietal patient with visual extinction and spatial neglect. Neuropsychologia, 40(12), 2156-2166.

Vuilleumier, P., Armony, J. L., Driver, J., \& Dolan, R. J. (2003). Distinct spatial frequency sensitivities for processing faces and emotional expressions. Nat Neurosci, 6(6), 624-631. doi: $10.1038 / \mathrm{nn} 1057$

Vuilleumier, P., \& Pourtois, G. (2007). Distributed and interactive brain mechanisms during emotion face perception: evidence from functional neuroimaging. Neuropsychologia, 45(1), 174-194. doi: 10.1016/j.neuropsychologia.2006.06.003

Vuilleumier, P., \& Schwartz, S. (2001). Emotional facial expressions capture attention. Neurology, 56(2), 153-158.

Weiner, K. S., \& Grill-Spector, K. (2011). Not one extrastriate body area: using anatomical landmarks, $\mathrm{hMT}+$, and visual field maps to parcellate limb-selective activations in human lateral occipitotemporal cortex. Neuroimage, 56(4), 2183-2199. doi: 10.1016/j.neuroimage.2011.03.041

Weiskrantz, L. (1986). Blindsight: A case study and implications.

Whalen, P. J., Kagan, J., Cook, R. G., Davis, F. C., Kim, H., Polis, S., .. . Johnstone, T. (2004). Human amygdala responsivity to masked fearful eye whites. Science, 306(5704), 2061. doi: 10.1126/science.1103617

Whalen, P. J., Rauch, S. L., Etcoff, N. L., Mclnerney, S. C., Lee, M. B., \& Jenike, M. A. (1998). Masked presentations of emotional facial expressions modulate amygdala activity without explicit knowledge. J Neurosci, 18(1), 411-418. 


\section{Chapter 1}

Williams, M. A., Morris, A. P., McGlone, F., Abbott, D. F., \& Mattingley, J. B. (2004). Amygdala responses to fearful and happy facial expressions under conditions of binocular suppression. J Neurosci, 24(12), 2898-2904. doi: 10.1523/JNEUROSCI.4977-03.2004

Yang, E., Brascamp, J., Kang, M. S., \& Blake, R. (2014). On the use of continuous flash suppression for the study of visual processing outside of awareness. Front Psychol, 5, 724. doi: 10.3389/fpsyg.2014.00724

Yang, E., Zald, D. H., \& Blake, R. (2007). Fearful expressions gain preferential access to awareness during continuous flash suppression. Emotion, 7(4), 882-886. doi: 10.1037/15283542.7.4.882

Yiend, J. (2010). The effects of emotion on attention: A review of attentional processing of emotional information. Cognition and Emotion, 24(1), 3-47. doi: 10.1080/02699930903205698

Yovel, G., \& Kanwisher, N. (2004). Face perception: domain specific, not process specific. Neuron, 44(5), 889-898. doi: 10.1016/j.neuron.2004.11.018

Yovel, G., \& Kanwisher, N. (2005). The neural basis of the behavioral face-inversion effect. Curr Biol, 15(24), 2256-2262. doi: 10.1016/j.cub.2005.10.072

Zilles, K., \& Clarke, S. (1997). Architecture, connectivity, and transmitter receptors of human extrastriate visual cortex Extrastriate cortex in primates (pp. 673-742): Springer. 


\section{Chapter 2}

The body as a tool for anger awareness - Differential effects of angry facial and bodily expressions on suppression from awareness

Corresponding manuscript:

Zhan, M., Hortensius, R., \& de Gelder, B. (2015). The Body as a Tool for Anger AwarenessDifferential Effects of Angry Facial and Bodily Expressions on Suppression from Awareness. PLoS One, 10(10), e0139768. 


\section{Chapter 2}

\section{Abstract}

Emotional signals are perceived whether or not we are aware of it. The evidence so far mostly came from studies with facial expressions. Here, we investigated whether the pattern of non-conscious face expression perception is found for whole body expressions.

Continuous flash suppression (CFS) was used to measure the time for neutral, fearful, and angry facial or bodily expressions to break from suppression. We observed different suppression time patterns for emotions depending on whether the stimuli were faces or bodies. The suppression time for anger was shortest for bodily expressions, but longest for the facial expressions. This pattern indicates different processing and detection mechanisms for faces and bodies outside awareness, and suggests that awareness mechanisms associated with dorsal structures might play a role in becoming conscious of angry bodily expressions. 


\section{Introduction}

In the course of daily interaction, people naturally display facial and bodily expressions that are easily recognized by others. Many studies have provided evidence that facial expressions can also be processed when they are not consciously perceived. For example, masked fearful faces orient spatial attention (Carlson \& Reinke, 2008), influence the perception of simultaneously presented unmasked expression (M. Tamietto \& de Gelder, 2008), elicit amygdala activation (Kim et al., 2010) and boost the connectivity between the amygdala and the colliculo-pulvinar pathway (Morris, Öhman, \& Dolan, 1999).

Traditionally, studies investigating non-conscious perception used visual masking techniques, as in the aforementioned studies. However, a recently developed paradigm called continuous flash suppression (CFS) (Tsuchiya \& Koch, 2005), a variant of binocular rivalry, is increasingly used to investigate the processing of emotions outside visual awareness. In this paradigm, one eye is presented with the experimental stimulus, while the other eye is presented with a flashing, colorful "Mondrian" pattern, which renders the stimulus invisible for the participant. This offers stronger suppression than masking, and creates a more stable non-conscious perception for participants (Yang, Brascamp, Kang, \& Blake, 2014). CFS exploits the rivalry of the dichoptic stimuli, hypothetically occurring at both monocular neurons in lower-level visual areas and binocular pattern selective neurons in higher visual areas (Tong, Meng, \& Blake, 2006). Previous studies using this paradigm have shown that facial stimuli with certain emotional contents can be processed without awareness (Jiang \& $\mathrm{He}, 2006)$, and that these emotional contents can affect judgments made by participants afterwards (Almeida, Pajtas, Mahon, Nakayama, \& Caramazza, 2013).

Although CFS can generally suppress visual stimuli for a relatively long time, the actual duration of suppression tends to vary with the stimulus category as well as with certain properties of the stimuli. In particular, stimuli that are salient or meaningful to the participant may break from suppression faster (Yang et al., 2014). This difference in suppression time between stimuli can be used to infer the differences in non-conscious processing before they reach awareness (Stein, Hebart, \& Sterzer, 2011). This breaking-fromsuppression (CFS-b) paradigm has been utilized to study processing differences of high-level features including emotions. For facial expressions of emotion, studies using fearful faces provided mixed results (Gray, Adams, Hedger, Newton, \& Garner, 2013; Jusyte, Mayer, Kunzel, Hautzinger, \& Schonenberg, 2014; Stein, Seymour, Hebart, \& Sterzer, 2014; Sterzer, Hilgenfeldt, Freudenberg, Bermpohl, \& Adli, 2011; Yang, Zald, \& Blake, 2007). Three of them showed that fearful faces tend to break from suppression faster than other emotional facial expressions (Gray et al., 2013; Stein et al., 2014; Yang et al., 2007), underscoring the attention-grabbing status of fearful facial expressions that was found in masking studies (Öhman, 2002).

The current human emotion theories may be incomplete due to an emphasis on the facial expressions; an important question is whether the effects obtained with facial expressions also generalize to bodily expressions of emotions. Bodily expressions are potent emotional signals when perceived consciously (B. de Gelder, 2006), as task-irrelevant information under attention manipulation (J. Van den Stock \& de Gelder, 2014), but also do so when 


\section{Chapter 2}

processed outside visual awareness. This has been demonstrated in some patients with cortical blindness due to visual cortex lesion, who show affective blindsight, the ability to still be able to process visual emotional signals (Beatrice De Gelder, Vroomen, Pourtois, \& Weiskrantz, 1999). For example, emotional bodies presented in the blind visual field elicited activations in subcortical structures including superior colliculus and pulvinar (J. Van den Stock et al., 2011), and elicited similar facial muscle reactions to the ones presented in the sighted visual field (M. Tamietto et al., 2009). Studies with neglect patients also showed that exposure to images with emotional bodies substantially reduced the attentional deficits ( $M$. Tamietto et al., 2015; Marco Tamietto, Geminiani, Genero, \& de Gelder, 2007). Together with masking studies in healthy participants (Hortensius, van Honk, de Gelder, \& Terburg, 2014; Stienen \& de Gelder, 2011), these studies provide evidence for the processing of bodily expressions of emotion outside awareness. In view of the fact that masking methods are not always conclusive (Kouider \& Dehaene, 2007), convergent evidence from other approaches is desirable.

The CFS paradigm has so far not been used to investigate non-conscious perception of emotions expressed by body postures. In the current study, we used the CFS-b paradigm to examine suppression time differences among stimuli with neutral, fearful, and angry emotions, conveyed by either the face or the body. Our study aimed to answer three questions. First, can CFS also be used to investigate non-conscious emotional body processing and if so, are there differences in suppression times of the different emotions? Second, is the pattern of suppression time differences between emotions similar for bodily and facial expressions? Finally, in view of a debate on this issue in the literature, can we add new evidence about the suppression times under CFS for emotional facial expressions?

\section{Methods}

\section{Participants}

Participants were recruited from the Maastricht University campus ( $n=32$, mean age $=21.7$, ranging from 18-30, with seven male and three left-handed participants). All participants had normal stereo and color vision, normal or corrected-to-normal visual acuity, and no history of neurological disorders. They provided written consent and received either credit points or monetary reward afterwards. The procedure for the study was approved by the Ethics Committee of Maastricht University, and was carried out in accordance with the standards established by the Declaration of Helsinki.

\section{Stimuli}

The stimuli were face and body images in grayscale. Each stimulus category had 24 identities, and each identity had a neutral, angry, and fearful expression. The face stimuli were adapted from the Radboud Faces Database (Langner et al., 2010). They were aligned at the eye level, and an ellipse (from below the chin to the top of the head, $1.91^{\circ} \times 2.67^{\circ}$ of visual angle) masked out the visual details for each facial stimulus outside that region. The body stimuli were adapted from Stienen and de Gelder (Stienen \& de Gelder, 2011), with facial information removed, and aligned at the shoe-level. Neutral stimuli consisted of individuals 
talking on the phone. The body stimuli spanned within $1.8^{\circ} \times 4.3^{\circ}$ visual angles. Since our interest lies more on the effects of different emotions within the same stimulus category (either face or body), and the emotions performed by the same individual were photographed under the same lighting conditions and camera settings, to keep the ecological validity, we used the full-contrast images without further balancing the luminance and contrast across emotions, or across stimulus category. Instead, the low-level properties were controlled by performing mixed-models analyses. Figure $1 \mathbf{A}$ shows examples of the stimuli used.

\section{Procedure}

The stimuli were presented in MATLAB (the MathWorks, Natick, MA, USA) with Psychtoolbox (Brainard, 1997; Pelli, 1997), on an LCD screen (Acer VG248, 3D capable, resolution $=1920 \times 1080$, refresh rate $=60 \mathrm{~Hz}$ ), in a room with dim light. The background of the screen was set to gray (RGB value $=128,128,128)$. In the middle of the screen, two rectangles ( $240 \times 160$ pixels, $6.4^{\circ} \times 4.27^{\circ}$ of visual angles) were placed side by side and the centers of the rectangles were 250 pixels apart (visual angle $=6.67^{\circ}$ ). A frame of 10 pixels delineated the border of the rectangles, and a black fixation cross was placed in the center of each rectangle. The fixation crosses and the frames helped to maintain convergence of the two rectangles for participants.

A chin rest was placed $59 \mathrm{~cm}$ away from the screen. Participants viewed the screen through a pair of diopter glasses (the diopter for each lens $=7$ ). The diopters were calculated and chosen according to the visual angle between the two rectangles (Schurger, 2009), which would shift each rectangle back to the center of screen upon viewing. A cardboard divider was placed between the screen and the chin rest, dividing the screen into two equal halves. Participants were instructed to fixate on the cross and free-fuse the two rectangles into one. The experiment would start only after participants reported that they could clearly see only one rectangle, and that the view was stable. All participants reported successful and stable fusion. With this setup, either eye of the participant could see only the content of the ipsilateral rectangle.

In a trial, a dynamic noise $(160 \times 240$ pixels, flashing at $10 \mathrm{~Hz})$ was projected into one of the rectangular frames. The noise images consisted of overlapping and colorful small rectangles (with height and width within $2^{\circ}$ ). The noise images in each trial were drawn randomly from 600 unique noise images. One stimulus imbedded in the same gray background was projected into the other rectangular frame, as the target stimulus. In each trial, the initial fixation cross changed to white one second before the start of the trial, and remained white throughout the trial. In the inter-trial intervals (length $=4 \mathrm{~s}$ ), the fixation cross changed back to black. Each trial had a duration of $9 \mathrm{~s}$. The stimulus was gradually ramped up from $0 \%$ contrast to full contrast in the first $8 \mathrm{~s}$, and remained at full contrast for another $1 \mathrm{~s}$. The contrast of the noise was ramped down in the first 8 seconds to $0 \%$. Thus in the 9 th second only the stimulus was presented in one of the rectangles. Participants were instructed to fixate continuously, to blink as little as possible during a trial, and to press the space bar on the keyboard as soon as they perceive anything else other than the dynamic noise. Figure1B presents the CFS-b procedure. We did not provide the participants with any prior knowledge about the nature of the suppressed stimuli in order to exclude possible top-down effects. 


\section{Chapter 2}

A
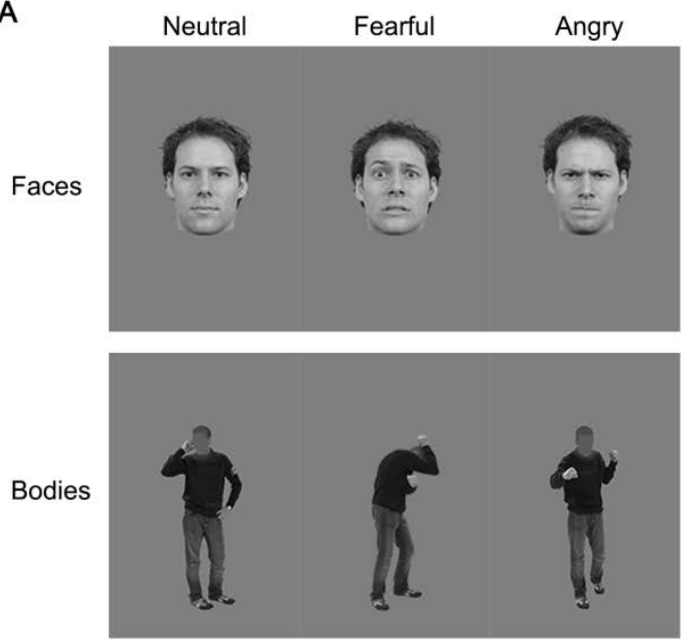

B

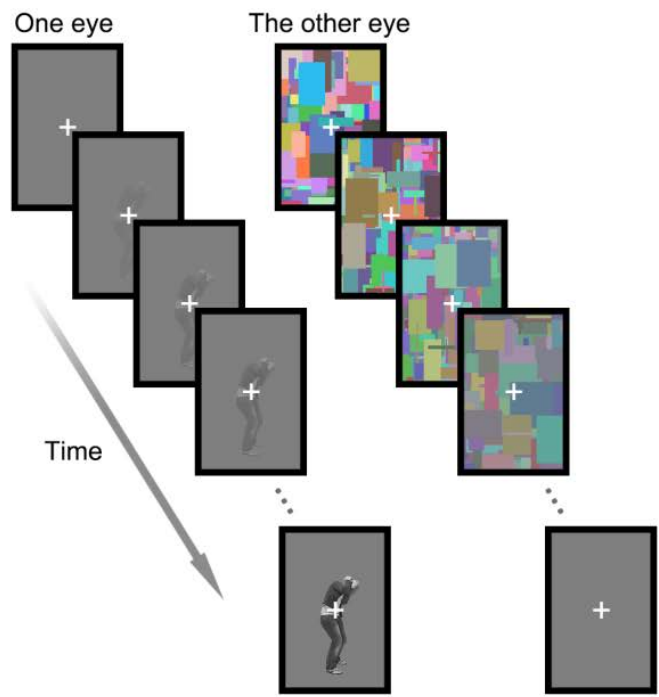

Figure 1. Examples of stimuli and the CFS-b procedure. (A) Examples of face and body stimuli, with neutral, fearful and angry emotional facial and bodily expressions were used. (B) The CFS-b procedure.

The face and body stimuli were presented in two separate blocks. The order of the blocks was counterbalanced between participants. Each individual stimulus was presented once in each eye. One block consisted of 144 trials, randomized within the block, and balanced between the eyes. In the middle of each block there was a short break.

After the experiment, participants were asked to free-recall the emotion categories that they recognized, and whether they clearly perceive the whole stimuli at the time they pressed the key. If they could not label the emotion of the body, they were asked to describe the posture.

\section{Main analyses}

The data were analyzed in SPSS and R (R Core Team, Vienna, Austria). For removing outliers, the single-trial suppression times were z-transformed for the data of each block separately, and also across the two blocks for each participant. The data points that exceed four standard deviations (SD) in either one of the z-transformations performed were excluded as outliers. Trials without a response (suppression time $\geq 9 \mathrm{~s}$ ) were also excluded. In total, $1.13 \%$ of the trials were excluded. In the face block, mean number of excluded trials was 1 out of 144 trials $(S D=1.29)$. In the body block, for one participant 28 trials were removed due to no response, while for the other participants mean number of excluded trials was 1 out of 144 trials, $S D=1.8$. There was no difference between the face and body blocks in the number of outliers removed, $t(31)=-0.351, p=.728$.

The raw suppression time (in seconds) was aggregated by participant. A two-way factorial repeated-measure ANOVA was performed, with category (face, body) and emotion (neutral, fear, anger) as within-subjects factors. Paired samples t tests were used for post-hoc testing. 


\section{Mixed models control analyses}

We also took low-level properties of the stimuli into consideration by performing mixed models analyses. Since we expect that the low-level properties will affect the two stimuli categories differently, the mixed models analyses were performed separately on the data of the face and the body blocks. As low-level properties, we counted the number of pixels for each body and face stimulus, and obtained the average pixel value for each. We also obtained the root-mean-square contrasts of the whole body stimuli, and of the eye-eyebrow region of the face stimuli.

The pixel count, mean pixel value, and the RMS contrasts were centered, and residualized on the emotion types using SPSS, and the residuals corresponded to the part not explained by the emotion types. The multicollinearity checks were performed for the face and the body data separately. The emotion type, with the residualized pixel number, mean pixel value, and RMS contrast were entered into a backward regression model as predictors. The suppression time was defined as the dependent variable. Multicollinearity checks showed a variance inflation factor below 1.5 for all the predictors, suggesting that they were free of multicollinearity problems. In the following paragraphs the "residualized" will be omitted for conciseness.

The mixed models analyses were performed in R (R Core Team, Vienna, Austria). The linear mixed models were constructed and compared with the Ime4 toolbox (Bates, Maechler, \& Bolker, 2012). For the models, fixed effect predictors included: emotion type, pixel count, mean pixel value, RMS contrast; random effects included: participant, stimulus, where the stimulus predictor is a by-item predictor for the stimuli. The details of model comparison steps are described in the result session. The least-squared mean estimates for the fixed effect emotion type was obtained using the Ismeans (Lenth, 2013) and pbkrtest (Halekoh \& Højsgaard, 2013) toolboxes. The models that differed in random effect predictors were fitted by the restricted maximum likelihood method (REML), and the models that differed in fixed effect predictors were fitted by the maximum likelihood method (ML). The final model was then fitted with REML to obtain the linear estimates.

\section{Results}

\section{Results of the main analyses}

There was a significant main effects of category, $F(1,31)=71.20, p<.000001, \eta_{p}^{2}=0.70$, and of emotion, $F(2,62)=8.47, p=.0006, \eta_{p}^{2}=0.21$. Importantly, there was a significant interaction between category and emotion, $F(2,62)=28.64, p<.001, \eta_{p}^{2}=0.48$.

Post-hoc paired-samples t-tests revealed that the suppression time for angry faces $(M=3.45$, $S E=0.21)$ was significantly longer than either neutral $(M=3.23, S E=0.19), t(31)=3.93, p<.001$, $r=0.57$, or fearful faces $(M=3.23, S E=0.18), t(31)=3.60, p=.001, r=0.54$. The difference between neutral and fearful facial expressions was not significant, $t(31)=0.04, p=.97, r=0.01$. 


\section{Chapter 2}

In contrast, suppression time for angry bodies was significantly shorter $(M=4.11, S E=0.21)$ compared to fearful $(M=4.58, S E=0.23)$, and neutral $(M=4.26, S E=0.20)$ bodies, with $t(31)=7.67, p<.001, r=0.81$ and $t(31)=2.63, p=.013, r=0.43$, respectively. Suppression time for fearful bodies was longer compared to neutral bodies, $t(31)=-4.29, p<.001, r=0.61$ (Figure2).
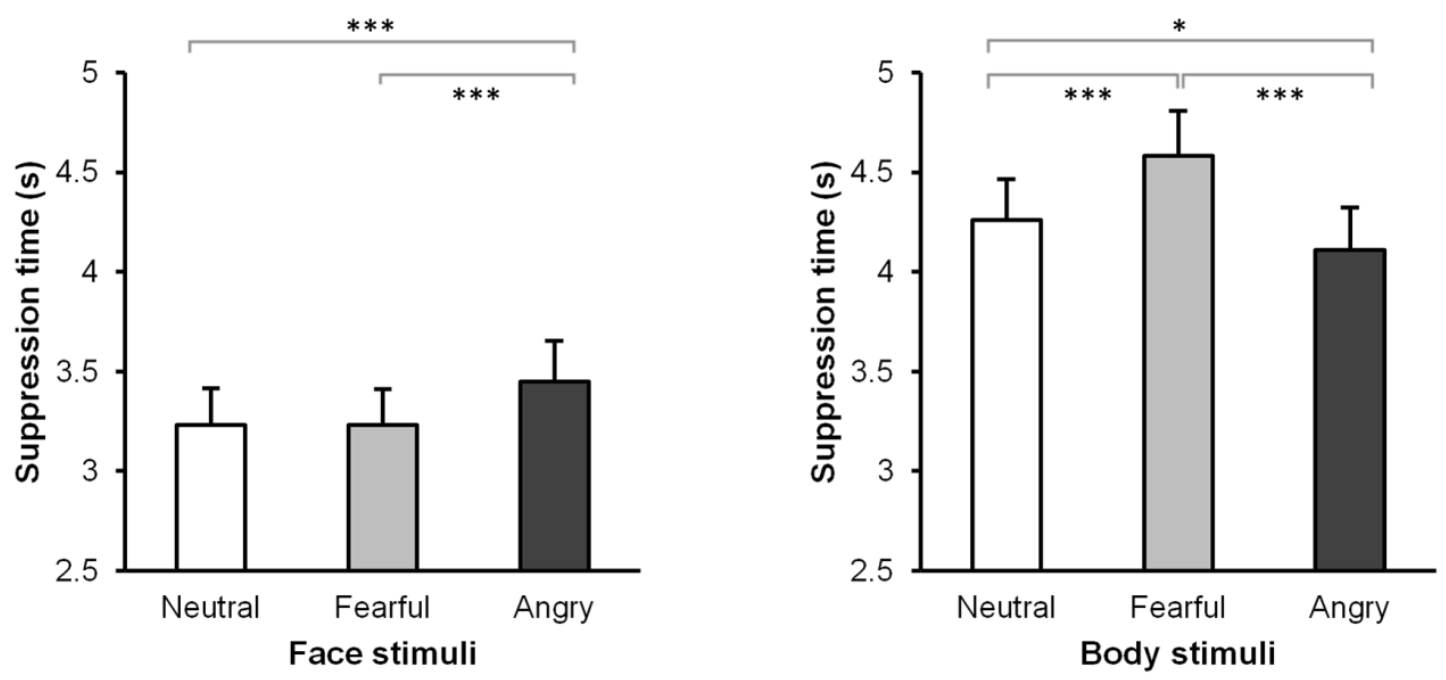

Figure2. Results of the face and body blocks. Suppression time for neutral, fearful, and angry facial and bodily expressions. Error bars represent standard errors. *: $p<.05 ;{ }^{* * *}: p<.001$.

To test if the order of the blocks influenced the results, we added order (face $\rightarrow$ body versus body $\rightarrow$ face) as a between-subjects factor in the repeated-measure ANOVA. There was no main effect of order, $F(1,30)=1.09, p=.31, \eta_{p}^{2}=0.04$, or emotion by order interaction, $F(2,60)=0.98, p=.38, \eta_{p}^{2}=0.03$. A significant interaction between category and order appeared, $F(1,30)=35.34, p=.000002, \eta_{\mathrm{p}}{ }^{2}=0.54$. The group with the body block following the face block ( $n=16, M=3.87, S E=0.28$ ) showed shorter suppression times for the body category compared to the group with the face block following the body block ( $n=16$, $M=4.76, S E=0.28), t(30)=-2.23, p=.03, r=0.38$. This was not the case for faces, $t(30)=0.24$, $p=.81, r=0.04$ (face $\rightarrow$ body: $M=3.35, S E=0.29$, and body $\rightarrow$ face, $M=3.26, S E=0.25$ ). Crucially, the three-way interaction between order, emotion and category was not significant, $F(2,60)=0.47, p=.63, \eta_{p}^{2}=0.02$. Order did not influence the main results.

In addition, we checked whether the participants based their decision on a consistent basis within each block, and if they didn't wait too long for a clear perception of the stimuli. All participants reported that they pressed the response button as soon as possible, but their partial-whole perception varied when pressing the button: 20 participants saw the whole stimuli most of the time, 7 participants saw partial features most of the time, and 5 participants had a mixed perception. To test if seeing the whole or partial of the stimuli affected the observed results, we added it as a between-subject factor in the repeatedmeasure ANOVA. There was no main effect of stimuli completeness, $F(2,29)=0.27, p=.77, \eta_{p}{ }^{2}$ $=0.02$. No interaction of stimuli completeness was present with either category, $F(2,29)=0.51, p=.60, \eta_{p}{ }^{2}=0.03$, or emotion, $F(2,29)=1.57, p=.19, \eta_{p}{ }^{2}=0.10$, or with category and emotion together, $F(4,58)=0.94, p=.45, \eta_{p}^{2}=0.06$. 


\section{Results of the mixed models control analyses}

\section{Face stimuli}

First, model 1 was constructed with one fixed effect predictor (emotion type), and two separate random effect predictors (participant, stimulus). An intercept and random slope for emotion type was included in the participant predictor. The model 1 had the maximal random effect structure justified by the data. A second model (model 2 ) excluded the random slope of emotion type in the participant predictor. Comparison of these two models showed that model 1 , with the random slope, better described the data, $\chi^{2}(5)=13.86, p=.016$. The random slope was kept in the subsequent models.

Model 3, 4 and 5 were constructed by adding a second fixed effect predictor, respectively: pixel count (model 3), mean pixel value (model 4), RMS contrast for the eye-eyebrow region (model 5). Model 5 with RMS contrast described the data better than model $1, \chi^{2}(1)=24.52$, $p<.001$. The estimate $(B)$ for the predictor RMS contrast was $-0.03, S E=0.005, t=-5.41$, $p=.0001$. The $p$ value was obtained using the pbkrtest toolbox, with 10000 simulation samples. Subsequently adding pixel count and mean pixel value to model 5 respectively wasn't justified by the $\chi^{2}$ change. The model 5 was selected as the final model.

In the final model, with emotion type and RMS contrast as fixed effect predictors, the least squared means of the contrasts also showed similar results to those in the ANOVA analysis, neutral versus fearful faces: $t(38.93)=1.88, p=.159$; neutral versus angry faces: $t(32.42)=-3.73$, $p=.002$, fearful versus angry faces: $t(39.40)=-4.99, p<.0001$. The estimate for fearful faces was $0.12 \mathrm{~s}$ shorter than neutral faces, which was a pattern also shown in the literature, but this difference was still not significant (Figure3 and Table 1).
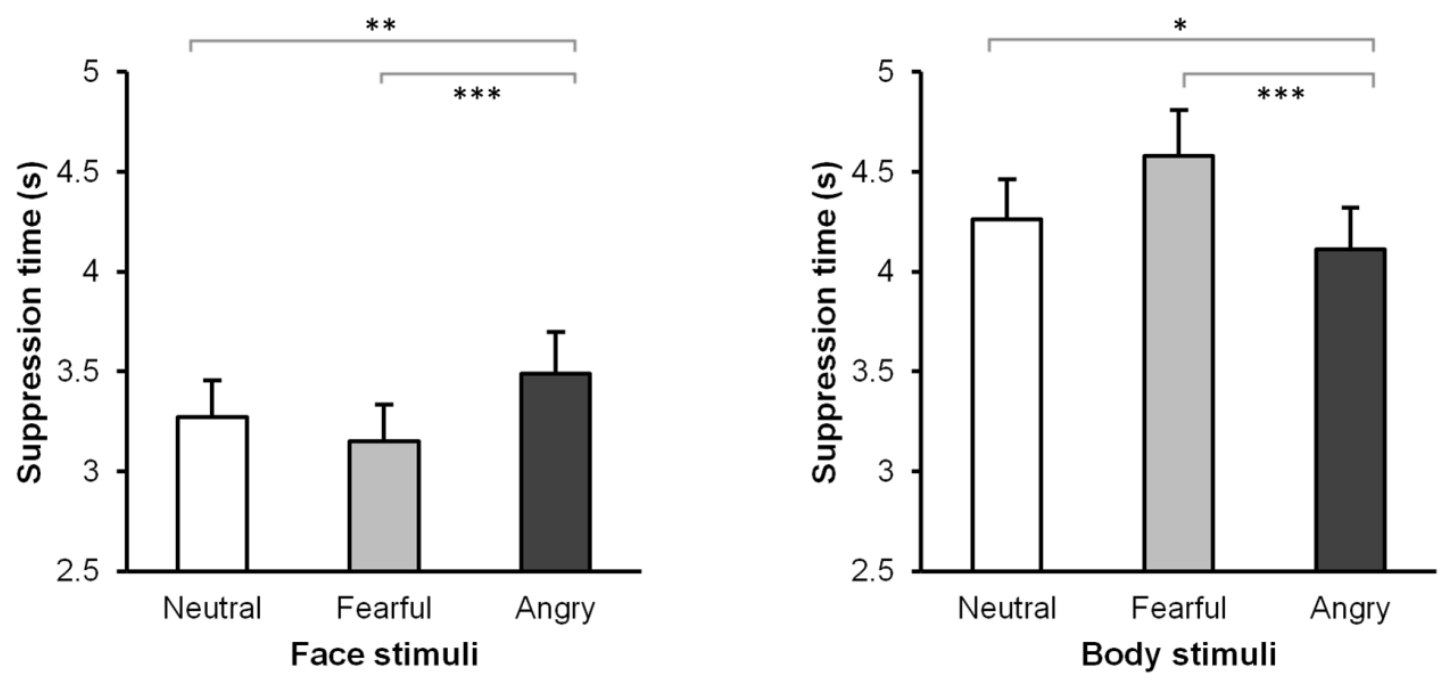

Figure3. Suppression time for neutral, fearful, and angry facial and bodily expressions after correcting for low-level features unrelated to emotion. Error bars represent standard errors. *: $p<.05 ; * *: p<.01 ; * * *: p<.001$. 


\section{Chapter 2}

Table 1. Estimates ( 6 values, in seconds), standard errors, and $t$ values for the fixed-effect predictors in the analyses for the face and body blocks.

\begin{tabular}{lccc}
\hline Predictor & Estimate $(s)$ & SE & $t$ \\
\hline Face stimuli & & & \\
Intercept (neutral faces) & 3.275 & 0.184 & 17.845 \\
Fearful vs. neutral faces & -0.120 & 0.062 & -1.923 \\
Angry vs. neutral faces & 0.216 & 0.056 & 3.834 \\
RMS Contrast of the eye region & -0.031 & 0.006 & -5.411 \\
& & & \\
Body stimuli & & & \\
Intercept (neutral bodies) & 4.264 & 0.202 & 21.099 \\
Fearful vs. neutral bodies & 0.317 & 0.083 & 3.846 \\
Angry vs. neutral bodies & 0.152 & 0.066 & -2.308 \\
Mean pixel value of the stimulus & 0.009 & 0.001 & 6.708 \\
\hline The $p$ values in the analyses were obtained by the Ismeans and pbkrtest toolboxes, thus were reported \\
separately in the text.
\end{tabular}

\section{Body stimuli}

The model comparisons for the body stimuli followed a similar procedure as those for the face stimuli. Model 1 and 2 were similarly constructed, with a random slope for emotion type in the participant predictor, either included (model 1) or omitted (model 2). Model 1 better described the data, $\chi^{2}(5)=14.76, p=.01$. The random effect structure was kept constant in the subsequent models.

In models 3, 4 and 5, comparing to model 1, pixel count (model 3), mean pixel value (model 4), RMS contrast (model 5) were added as the second fixed effect predictor, respectively. Comparisons showed that in the 3 models, model 4 with the mean pixel value for the whole body stimulus described the data significantly better than model $1, \chi^{2}(0)=34.84, p<.001$. The estimate ( 6 ) for the predictor mean pixel value was $0.009, \mathrm{SE}=0.001, t=6.58, p=.0001$ (The $p$ value was obtained by comparing model 5 with model 1 , using a parametric bootstrapping model comparison test in the pbkrtest toolbox, with 10000 simulation samples).

Subsequently, adding pixel count and RMS contrast to model 4 respectively, was not justified by the $\chi^{2}$ change. The model 4 was selected as the final model.

In the final model, with emotion type and mean pixel value as fixed effect predictors, the least squared means of the contrasts showed a similar pattern for the emotion as in the ANOVA analysis: neutral versus fearful bodies: $t(41.69)=-3.76, p=.0015$; neutral versus angry bodies: $t(38.57)=2.26, p=.073$, fearful versus angry bodies: $t(40.28)=6.51, p<.0001$ (Figure3 and Table 1).

\section{Discussion}

Our goal was threefold: 1 ) to use the same CFS-b paradigm to investigate perception of bodily expressions without visual awareness, 2 ) to investigate the possible differences in 
pattern of suppression time differences among emotions for face and body stimuli, and 3) to add to the available evidence on the perception of facial expressions using the CFS-b paradigm.

First, we showed that the suppression times did differ among bodily expressions. Specifically, angry bodies broke suppression faster, while fearful bodies broke suppression slower. Compared to fearful bodies, angry bodies represent a much more direct threat to the observer, which tend to trigger avoidance and escape behaviors (Pichon, de Gelder, \& Grezes, 2009). Consistent with this notion, previous fMRI studies showed increased activity in the anterior temporal lobe, the premotor cortex, and the ventromedial prefrontal cortex, suggesting automatic defense-related action preparation, in response to angry bodies (Pichon, de Gelder, \& Grezes, 2008; Pichon et al., 2009). This involvement of the actionpreparation network might be the mechanism that facilitated the breaking from suppression for angry bodies in our study. The posterior superior temporal sulcus might also play a role, as applying transcranial magnetic stimulation to this region facilitated the detection of changes in the masked angry bodies but not neutral bodies (M. Candidi, Stienen, Aglioti, \& de Gelder, 2011).

For facial expressions, our results showed that angry faces broke from suppression slower than neutral and fearful faces. This is in accordance with the study of Gray et al. (Gray et al., 2013), showing that angry faces broke from suppression slower than neutral faces. Our result did not show a significant difference between fearful and neutral faces. While several CFS-b studies with fearful faces showed a shorter suppression time than neutral faces (Gray et al., 2013; Stein et al., 2014; Yang et al., 2007), two other studies showed a non-significant difference in their healthy controls (Jusyte et al., 2014; Sterzer et al., 2011). This discrepancy might be caused by the large number of identities ( 24 identities) in the facial stimuli used in the current study, compared to the number ( 4 or 8 identities) in the aforementioned studies. The large number of stimulus identities here introduced ecological validity, but may also have introduced variability in the responses of the participants. In general, the pattern of suppression time among the three facial expressions shown in our results does not depart from that in the literature. Further research is needed to better understand the role of individual differences in conscious and non-conscious fear perception. For example, individual differences in sensitivity have been shown for fearful (Doty, Japee, Ingvar, \& Ungerleider, 2014) and angry expressions (Hortensius et al., 2014).

Finally, our results also provide evidence, regarding the suppression time patterns among emotions and possible differences between facial and bodily expressions. Our results already showed a general suppression time difference between faces and bodies, with bodies being suppressed longer. This is consistent with the literature (Stein, Sterzer, \& Peelen, 2012), and indicated a difference in non-conscious processing of faces and bodies. The difference in the pattern obtained for face and bodily expressions may therefore indicate that a degree of category specificity may be present at the level of non-conscious emotion signal processing.

Notwithstanding the fact that face and bodily expressions may convey the same meaning, they are actually very different visual stimuli once one gets away from asking subjects for explicit cognitive recognition of the emotion. At the level of visual features, faces and bodies 


\section{Chapter 2}

clearly convey the emotional information by very different means. The facial emotion is conveyed by the fine details of internal facial features, including the salient eye-eyebrow region, which is thought to be driving the detection of faces under CFS (Gray et al., 2013; Yang et al., 2007). There is evidence pointing to eye-specific mechanisms for face perception, in which the amygdala may play an important role: eye contact alone activated the amygdala for a complete cortical blind patient (Burra et al., 2013), single-cell recordings in monkey amygdala also found specialized cells for eye contact (Mosher, Zimmerman, \& Gothard, 2014). The bodily emotion expression is rather conveyed by the position and movements of the body parts leading to better expression recognition at a further distance.

From the vantage point of basic emotion theory, it has been shown that the same brain areas are being activated by the same emotion, whether the stimuli are faces, bodies or voices (Peelen, Atkinson, \& Vuilleumier, 2010). However, it is not clear so far whether the categorical structure and the representation of the emotion labels is the same at earlier processing levels, especially at a non-conscious level (B de Gelder, 2016; LeDoux, 2012). Studies comparing the neural basis showed that emotional face and body processing involved overlapping brain regions, including the fusiform gyrus, superior temporal gyrus, and the middle occipital gyrus; however the calcarine sulcus, cerebellum, superior frontal gyrus and anterior cingulate gyrus were more involved in face processing, while the superior occipital gyrus, parieto-occipital sulcus and the intraparietal sulcus were more involved in body processing (B. de Gelder et al., 2010).

These processing differences may indicate stimulus and emotion selective interactions with the parietal attention network (Colby \& Goldberg, 1999) for face and body stimuli, thus contributing to different patterns of faces and bodies in reaching awareness. For bodily expressions, studies showed that activation increases in the motor system (Avenanti, Candidi, \& Urgesi, 2013). Bodily expressions may trigger the involvement of more actionperception and -preparation processes (M Candidi, Aglioti, \& de Gelder, 2015; M. Candidi et al., 2011; B. de Gelder, 2006; Goldberg, Preminger, \& Malach, 2014). This may be an important part of the mechanism underlying the shorter suppression time for angry bodies in our study.

A complementary explanation may be provided by processes related to proprioception. A recent CFS study showed that when participants saw a suppressed hand image in the same pose as their actual hand, the suppressed hand broke suppression faster (Salomon, Lim, Herbelin, Hesselmann, \& Blanke, 2013). This might indicate that the matching of the proprioceptive information and the unconsciously observed visual information may have a facilitatory effect for stimuli to reach awareness (Salomon et al., 2013). Another recent study comparing neural correlates of seen and unseen bodily expressions argues in favor of the role of the insula and sensorimotor processes in the transition from non-conscious perception to awareness (M. Tamietto et al., 2015). The results indicated that the integration between the mapping of bodily changes at the neural level and the peripheral arousal is critical for the conscious visual experience of emotional signals (M. Tamietto et al., 2015). 
Apart from the category-specific differences between the stimuli, the depth of suppression with the CFS paradigm is related to the spatial and temporal properties of both the stimuli and the masks, such as contrasts (Yang \& Blake, 2012). Our research questions mainly focused on the different patterns elicited by the same emotions in faces and bodies, rather than the absolute suppression time between the two categories. The spatial-temporal effects of the stimuli and the masks are compatible within each category. Still, could the present results be explained by these low level properties of the stimuli, and could this factor thus, at least indirectly, be responsible for the observed asymmetric patterns between faces and bodies? For face stimuli, three studies showed that fearful faces broke from suppression faster than neutral faces (Gray et al., 2013; Stein et al., 2014; Yang et al., 2007). Two of them suggested that faster access was caused mainly by low-level features of the eye and eyebrow region (e.g. the local high contrast in the region), but not by the emotional information (Gray et al., 2013; Yang et al., 2007). Here we used a large number of different identities in our stimuli (24 identities for both face and body stimuli), introducing variability in the lower-level visual properties. To further control for the effect of the low-level features of the stimuli, we did an analysis that residualized the low-level features on the emotion types, and separated the effect of a low-level feature into two parts: one part that is systematically introduced by the stereotypical features relating to specific emotion types (such as the lowered RMS contrast in the eye-eyebrow region for fearful faces, caused by the larger area of eye whites, which is related to possible eye-specific mechanisms), and a second part that is pure low-level variances (the residuals, which might include the influence of the facial skin tone variation across individuals that is not related to the emotion). We examined whether this second part would be important in affecting the suppression time. For both the body and face stimuli, the suppression time was indeed influenced by the lower-level visual properties of the stimuli, but only to a very small extent. For the face and body data respectively, the RMS contrast of the eye-eyebrow region and the mean pixel value for the whole stimulus described the data well, but the estimate was one to two orders of magnitude smaller than that for the emotion type. These results indicated that in the current study, the variance of the low-level feature unrelated to the emotions was not necessarily the dominant factor for the effect observed in CFS experiments. The difference between the emotion contents, which was processed at a higher level, was still the biggest factor for the difference in suppression times.

\section{Conclusion}

We examined the suppression time under CFS for neutral, fearful and angry facial and bodily expressions. Our results showed that fearful and angry bodily expressions differ in suppression time, with angry bodies breaking from suppression faster, suggesting a different involvement of the action perception network. In contrast, angry faces broke from suppression more slowly comparing to the other two facial expressions. These results indicate different mechanisms and neural networks for non-conscious perception of emotional faces and bodies. Future fMRI studies are needed to further explain the processing mechanisms for these emotions under non-conscious viewing conditions. 


\section{Chapter 2}

\section{References}

Almeida, J., Pajtas, P. E., Mahon, B. Z., Nakayama, K., \& Caramazza, A. (2013). Affect of the unconscious: visually suppressed angry faces modulate our decisions. Cogn Affect Behav Neurosci, 13(1), 94-101. doi: 10.3758/s13415-012-0133-7

Avenanti, A., Candidi, M., \& Urgesi, C. (2013). Vicarious motor activation during action perception: beyond correlational evidence. Front Hum Neurosci, 7, 185. doi: 10.3389/fnhum.2013.00185

Bates, D., Maechler, M., \& Bolker, B. (2012). Ime4: Linear mixed-effects models using S4 classes.

Brainard, D. H. (1997). The psychophysics toolbox. Spatial vision, 10, 433-436.

Burra, N., Hervais-Adelman, A., Kerzel, D., Tamietto, M., de Gelder, B., \& Pegna, A. J. (2013). Amygdala activation for eye contact despite complete cortical blindness. J Neurosci, 33(25), 1048310489. doi: 10.1523/JNEUROSCI.3994-12.2013

Candidi, M., Aglioti, S., \& de Gelder, B. (2015). Virtual lesion of right posterior superior temporal sulcus modulates conscious visual perception of fearful expressions in faces and bodies. Cortex.

Candidi, M., Stienen, B. M., Aglioti, S. M., \& de Gelder, B. (2011). Event-related repetitive transcranial magnetic stimulation of posterior superior temporal sulcus improves the detection of threatening postural changes in human bodies. J Neurosci, 31(48), 17547-17554. doi: 10.1523/JNEUROSCI.0697-11.2011

Carlson, J. M., \& Reinke, K. S. (2008). Masked fearful faces modulate the orienting of covert spatial attention. Emotion, 8(4), 522-529. doi: 10.1037/a0012653

Colby, C. L., \& Goldberg, M. E. (1999). Space and attention in parietal cortex. Annu Rev Neurosci, 22(1), 319-349.

de Gelder, B. (2006). Towards the neurobiology of emotional body language. Nature Reviews Neuroscience, 7(3), 242-249.

de Gelder, B. (2016). Emotional Body Perception in the Wild. In L. F. Barrett (Ed.), Handbook of Emotions. New York, NY: The Guilford Press.

de Gelder, B., Van den Stock, J., Meeren, H. K., Sinke, C. B., Kret, M. E., \& Tamietto, M. (2010). Standing up for the body. Recent progress in uncovering the networks involved in the perception of bodies and bodily expressions. Neurosci Biobehav Rev, 34(4), 513-527. doi: 10.1016/j.neubiorev.2009.10.008

De Gelder, B., Vroomen, J., Pourtois, G., \& Weiskrantz, L. (1999). Non-conscious recognition of affect in the absence of striate cortex. Neuroreport, 10(18), 3759-3763.

Doty, T. J., Japee, S., Ingvar, M., \& Ungerleider, L. G. (2014). Intersubject variability in fearful face processing: the link between behavior and neural activation. Cogn Affect Behav Neurosci, 14(4), 1438-1453. doi: 10.3758/s13415-014-0290-y

Goldberg, H., Preminger, S., \& Malach, R. (2014). The emotion-action link? Naturalistic emotional stimuli preferentially activate the human dorsal visual stream. Neuroimage, 84, 254-264. doi: 10.1016/j.neuroimage.2013.08.032

Gray, K. L., Adams, W. J., Hedger, N., Newton, K. E., \& Garner, M. (2013). Faces and awareness: lowlevel, not emotional factors determine perceptual dominance. Emotion, 13(3), 537-544. doi: $10.1037 / \mathrm{a} 0031403$

Halekoh, U., \& Højsgaard, S. (2013). pbkrtest: Parametric bootstrap and Kenward Roger based methods for mixed model comparison. $R$ package version 0.3-8, URL http://CRAN. R-project. org/package $=$ pbkrtest.

Hortensius, R., van Honk, J., de Gelder, B., \& Terburg, D. (2014). Trait dominance promotes reflexive staring at masked angry body postures. PLoS One, 9(12), e116232. doi: 10.1371/journal.pone.0116232 


\section{Breaking from CFS on facial and bodily emotions}

Jiang, Y., \& He, S. (2006). Cortical responses to invisible faces: dissociating subsystems for facialinformation processing. Curr Biol, 16(20), 2023-2029. doi: 10.1016/j.cub.2006.08.084

Jusyte, A., Mayer, S. V., Kunzel, E., Hautzinger, M., \& Schonenberg, M. (2014). Unemotional traits predict early processing deficit for fearful expressions in young violent offenders: an investigation using continuous flash suppression. Psychol Med, 1-13. doi: 10.1017/S0033291714001287

Kim, M. J., Loucks, R. A., Neta, M., Davis, F. C., Oler, J. A., Mazzulla, E. C., \& Whalen, P. J. (2010). Behind the mask: the influence of mask-type on amygdala response to fearful faces. Soc Cogn Affect Neurosci, 5(4), 363-368. doi: 10.1093/scan/nsq014

Kouider, S., \& Dehaene, S. (2007). Levels of processing during non-conscious perception: a critical review of visual masking. Philos Trans R Soc Lond B Biol Sci, 362(1481), 857-875. doi: $10.1098 /$ rstb.2007.2093

Langner, O., Dotsch, R., Bijlstra, G., Wigboldus, D. H., Hawk, S. T., \& van Knippenberg, A. (2010). Presentation and validation of the Radboud Faces Database. Cognition and Emotion, 24(8), 1377-1388.

LeDoux, J. (2012). Rethinking the emotional brain. Neuron, 73(4), 653-676. doi: 10.1016/j.neuron.2012.02.004

Lenth, R. V. (2013). Ismeans: Least-squares means. R package version 1.06-05.

Morris, J. S., Öhman, A., \& Dolan, R. J. (1999). A subcortical pathway to the right amygdala mediating "unseen" fear. Proceedings of the National Academy of Sciences, 96(4), 1680-1685.

Mosher, C. P., Zimmerman, P. E., \& Gothard, K. M. (2014). Neurons in the monkey amygdala detect eye contact during naturalistic social interactions. Curr Biol, 24(20), 2459-2464. doi: 10.1016/j.cub.2014.08.063

Öhman, A. (2002). Automaticity and the amygdala: Nonconscious responses to emotional faces. Current directions in psychological science, 11(2), 62-66.

Peelen, M. V., Atkinson, A. P., \& Vuilleumier, P. (2010). Supramodal representations of perceived emotions in the human brain. The Journal of neuroscience, 30(30), 10127-10134.

Pelli, D. G. (1997). The VideoToolbox software for visual psychophysics: Transforming numbers into movies. Spatial vision, 10(4), 437-442.

Pichon, S., de Gelder, B., \& Grezes, J. (2008). Emotional modulation of visual and motor areas by dynamic body expressions of anger. Soc Neurosci, 3(3-4), 199-212. doi: 10.1080/17470910701394368

Pichon, S., de Gelder, B., \& Grezes, J. (2009). Two different faces of threat. Comparing the neural systems for recognizing fear and anger in dynamic body expressions. Neuroimage, 47(4), 1873-1883. doi: 10.1016/j.neuroimage.2009.03.084

Salomon, R., Lim, M., Herbelin, B., Hesselmann, G., \& Blanke, O. (2013). Posing for awareness: proprioception modulates access to visual consciousness in a continuous flash suppression task. J Vis, 13(7), 2. doi: 10.1167/13.7.2

Schurger, A. (2009). A very inexpensive MRI-compatible method for dichoptic visual stimulation. J Neurosci Methods, 177(1), 199-202.

Stein, T., Hebart, M. N., \& Sterzer, P. (2011). Breaking Continuous Flash Suppression: A New Measure of Unconscious Processing during Interocular Suppression? Front Hum Neurosci, 5, 167. doi: 10.3389/fnhum.2011.00167

Stein, T., Seymour, K., Hebart, M. N., \& Sterzer, P. (2014). Rapid fear detection relies on high spatial frequencies. Psychol Sci, 25(2), 566-574. doi: 10.1177/0956797613512509

Stein, T., Sterzer, P., \& Peelen, M. V. (2012). Privileged detection of conspecifics: evidence from inversion effects during continuous flash suppression. Cognition, 125(1), 64-79. doi: 10.1016/j.cognition.2012.06.005 


\section{Chapter 2}

Sterzer, P., Hilgenfeldt, T., Freudenberg, P., Bermpohl, F., \& Adli, M. (2011). Access of emotional information to visual awareness in patients with major depressive disorder. Psychol Med, 41(8), 1615-1624. doi: 10.1017/S0033291710002540

Stienen, B. M., \& de Gelder, B. (2011). Fear detection and visual awareness in perceiving bodily expressions. Emotion, 11(5), 1182-1189. doi: 10.1037/a0024032

Tamietto, M., Castelli, L., Vighetti, S., Perozzo, P., Geminiani, G., Weiskrantz, L., \& de Gelder, B. (2009). Unseen facial and bodily expressions trigger fast emotional reactions. Proc Natl Acad Sci U S A, 106(42), 17661-17666. doi: 10.1073/pnas.0908994106

Tamietto, M., Cauda, F., Celeghin, A., Diano, M., Costa, T., Cossa, F. M., . . de de Gelder, B. (2015). Once you feel it, you see it: Insula and sensory-motor contribution to visual awareness for fearful bodies in parietal neglect. Cortex, 62, 56-72. doi: 10.1016/j.cortex.2014.10.009

Tamietto, M., \& de Gelder, B. (2008). Affective blindsight in the intact brain: neural interhemispheric summation for unseen fearful expressions. Neuropsychologia, 46(3), 820-828. doi: 10.1016/j.neuropsychologia.2007.11.002

Tamietto, M., Geminiani, G., Genero, R., \& de Gelder, B. (2007). Seeing fearful body language overcomes attentional deficits in patients with neglect. J Cogn Neurosci, 19(3), 445-454.

Tong, F., Meng, M., \& Blake, R. (2006). Neural bases of binocular rivalry. Trends Cogn Sci, 10(11), $502-$ 511. doi: 10.1016/j.tics.2006.09.003

Tsuchiya, N., \& Koch, C. (2005). Continuous flash suppression reduces negative afterimages. Nat Neurosci, 8(8), 1096-1101. doi: 10.1038/nn1500

Van den Stock, J., \& de Gelder, B. (2014). Face identity matching is influenced by emotions conveyed by face and body. Front Hum Neurosci, 8, 53. doi: 10.3389/fnhum.2014.00053

Van den Stock, J., Tamietto, M., Sorger, B., Pichon, S., Grézes, J., \& de Gelder, B. (2011). Corticosubcortical visual, somatosensory, and motor activations for perceiving dynamic whole-body emotional expressions with and without striate cortex (V1). Proceedings of the National Academy of Sciences, 108(39), 16188-16193.

Yang, E., \& Blake, R. (2012). Deconstructing continuous flash suppression. J Vis, 12(3), 8. doi: 10.1167/12.3.8

Yang, E., Brascamp, J., Kang, M. S., \& Blake, R. (2014). On the use of continuous flash suppression for the study of visual processing outside of awareness. Front Psychol, 5, 724. doi: 10.3389/fpsyg.2014.00724

Yang, E., Zald, D. H., \& Blake, R. (2007). Fearful expressions gain preferential access to awareness during continuous flash suppression. Emotion, 7(4), 882-886. doi: 10.1037/1528-

3542.7.4.882

\section{S1 Data. Data of the CFS-b experiment.}

DOI: 10.1371/journal.pone.0139768 


\section{Chapter 3}

\section{Continuous flash suppression induces strong suppression of visual stimulus information}

Corresponding manuscript for experiment 3:

Zhan, M., \& de Gelder, B. (In press). Unconscious fearful body expression perception enhances discrimination of conscious anger expressions under continuous flash suppression. Neuropsychologia. 


\section{Chapter 3}

\section{Abstract}

The continuous flash suppression (CFS) paradigm has been increasingly used to study unconscious visual perception. To compare to studies in patients with brain lesions, and to backward masking studies in healthy participants, we rendered emotional face and body stimuli invisible in three behavioral experiments. We found that CFS in general induced very strong suppression of the stimulus information. In the first experiment, we did not observe an affective priming effect for fearful and happy faces and bodies. In the second experiment, we did not observe dissociation between the detection performance and confidence ratings for angry and neutral bodies. In the third experiment with bilateral presentation of fearful and angry bodies, the CFS paradigm did not allow a redundant target effect to occur, which would facilitate responses for congruent unconscious emotional bodies; instead we found a facilitation effect on reaction times induced by the body stimuli of incongruent emotions, showing similarities to hemianopia patients without blindsight effects. These results indicate that unconscious visual processing is not one single phenomenon, but is likely to involve multiple mechanisms, processes and brain regions. Further studies on neural signatures of unconscious processing are necessary. 


\section{Introduction}

The research of unconscious visual perception is a fascinating topic. To study it, finding a measure that dissociates between behavioral performance and subjective consciousness is the most used research strategy. In finding dissociations, studies of patients with brain lesions have been contributing to our understanding of unconscious processes in the intact brain. For blindsight patients with V1 lesions, they lost their visual awareness due to the lesion, and could not report the presence of a visual target, but they could still react to the visual target above chance level (Danckert \& Rossetti, 2005; Weiskrantz, 1986). Perception without awareness in blindsight patients was also reported for emotional stimuli (de Gelder, Vroomen, Pourtois, \& Weiskrantz, 1999). For neglect patients, usually caused by parietal lesions, they would not consciously perceive a contralesional stimulus when another salient target was present in the ipsilesional visual field, unless explicitly asked to direct their attention to the contralesional side (Corbetta \& Shulman, 2011; Driver \& Vuilleumier, 2001). This makes the phenomenon of vision without awareness much more complex than assumed in the distinction between conscious and non-conscious perception. At present it is not clear whether a single distinction of conscious versus non-conscious perception applies across a large range of stimulus conditions (low level, high level vision, affective or neutral images), and across different kinds of patients and lesion locations (neglect or blindsight), for different methods of making images invisible in neurologically intact participants (masking, binocular rivalry, continuous flash suppression) and for different experimental techniques and associated response measurements (direct or indirect effect).

Because patients with lesions are rare, several methodological paradigms have been applied to study unconscious processes in healthy participants. The most widely used of them is the masking paradigm, with which the subjective percept of the target stimulus is disrupted, by a mask presented before (forward masking) or after (backward masking) it, or on both time points (sandwich masking). However, the strength of masking does not fully render stimuli being subliminal (Kouider \& Dehaene, 2007). Another method is binocular rivalry, which utilizes the interocular competition of two dichoptically presented stimuli, and the percept for one of them would be disrupted by the other (Tong, Meng, \& Blake, 2006). However, the duration of the stimulus dominance was not stable, and was not freely controllable by the participant. Another recently developed method is continuous flash suppression (CFS). Utilizing interocular competitions similar to binocular rivalry, the subjective percept of a lowcontrast target stimulus in one eye could be suppressed by a high-contrast and dynamic noise pattern in the other eye. Compared to backward masking and binocular rivalry, CFS has stronger suppression strength, and could reliably render a stimulus invisible for a few seconds (Tsuchiya \& Koch, 2005; Tsuchiya, Koch, Gilroy, \& Blake, 2006).

With healthy participants, masking and CFS paradigms have been applied to study unconscious emotional perception. With the masking paradigm, following a paradigm that established dissociation between confidence ratings at two stimulus onset asynchrony time points with similar detection performance (Lau \& Passingham, 2006), a comparison of bodies expressing fear, anger and happiness showed that, the detection performance (measured by $d^{\prime}$ ) and the confidence rating co-varied less for the fearful bodies (Stienen \& de Gelder, 


\section{Chapter 3}

2011). When fearful and happy faces were bilaterally presented, with one visual field masked to emulate blindsight-like effects in healthy participants, the masked faces with congruent emotion to the visible faces showed a redundant target effect: a shortening of the RT (Tamietto \& de Gelder, 2008). With the CFS paradigm, blindsight-like percepts, and dissociations between neural activity and percept have been found for emotional stimuli with healthy participants. For example, fearful faces have been found to break from CFsuppression (b-CFS) and enter into awareness faster than neutral and happy faces (Yang, Zald, \& Blake, 2007), and could induce amygdala activation when suppressed under CFS (Jiang \& He, 2006). When using the affective priming paradigm, angry invisible faces would prime and lower the likability for the target neutral Chinese characters, both with the backward masking and the CFS paradigm (Almeida, Pajtas, Mahon, Nakayama, \& Caramazza, 2013).

The redundant target effect is an indirect effect measurement that has shown useful to assess non-conscious perception. It has been established initially in healthy participants under conscious viewing conditions: when bilaterally presenting two targets, the reaction times (RTs) are shorter comparing to one-target conditions (Miller, 1982; Raab, 1962). Since the loss of visual awareness for these patients is visual field-specific, presenting a redundant stimulus in the blind visual field in addition to a stimulus in the intact visual field has been used to study the patients' visual processing, especially for emotional information conveyed by human faces. A redundant target effect had been found in both blindsight patients and neglect patients, that the processing of target emotional stimuli was facilitated by invisible stimuli with congruent emotions, similar to the effect for healthy participants under conscious viewing conditions (Tamietto, Latini Corazzini, de Gelder, \& Geminiani, 2006). With blindsight patients, previous studies found that the RTs for detecting the emotional faces (fear and sad) in the intact visual field were shortened by the faces in the blind field with congruent emotions (de Gelder, Morris, \& Dolan, 2005; de Gelder, Pourtois, van Raamsdonk, Vroomen, \& Weiskrantz, 2001). With neglect patients, emotional faces were extinguished less than neutral faces (Vuilleumier \& Schwartz, 2001). When extinguished, fearful faces activated amygdala similar to when they were visible (Vuilleumier et al., 2002). When priming a visible target face (happy, sad) with extinguished emotional faces, primes with congruent emotion to the target elicited faster RTs than incongruent emotions (Williams \& Mattingley, 2004). However, with hemianopia patients that had unilateral lesions but did not show any classical blindsight effects, studies did not find a redundant target effect, instead found a facilitation effect of fearful faces in the blind visual field, for detecting happy faces in the intact visual field and enhancing its N170 component of ERP (Bertini, Cecere, \& Ladavas, 2013; Cecere, Bertini, Maier, \& Ladavas, 2014). They found this facilitation effect was even present for non-emotional tasks, including gender discrimination and orientation discrimination of Gabor patches in the intact visual field (Bertini et al., 2013; Bertini, Cecere, \& Ladavas, 2017).In two of the three studies, the facilitation was found only for patients with left hemispheric lesions (Bertini et al., 2017; Cecere et al., 2014).

Similar to faces, the human body is also a category conveying information of identity and emotion. Because of the behavioral relevance, body stimuli have also been used as an effective tool to probe unconscious visual processing. For blindsight patients, both neutral body and face stimuli induced BOLD activation in the superior temporal sulcus and the 
amygdala (Van den Stock et al., 2014). When presented to the blind field, happy and fearful bodies and faces could both trigger fast facial muscle and pupillary reactions (Tamietto et al., 2009), angry dynamic body expressions could activate not only primary somatosensory, motor and premotor areas, but also in bilateral superior colliculi, pulvinar, amygdala and the right fusiform gyrus (Van den Stock et al., 2011). For neglect patients, when two stimuli were presented simultaneously in the two visual fields, bodies expressing fear were less extinguished than bodies expressing happiness, when presented to the contralesional visual field, showing an attention-grabbing effect (Tamietto, Geminiani, Genero, \& de Gelder, 2007). Extinguished fearful bodies also induced activation in extrastriate body areas and the left amygdala (Tamietto et al., 2015).

To further establish the study of unconscious emotional body processing with healthy participants, and compare to both previous masking and patient studies, we applied CFS with a series of emotional body stimuli in 3 behavioral experiments. In the first experiment with the affective priming paradigm, we compared the priming effects of fearful and happy faces and bodies, because these emotional stimuli were found to trigger fast facial and pupillary reactions in blindsight patients (Tamietto et al., 2009). In the second experiment, we examined the detection performance and confidence ratings for angry and neutral bodies. In the third experiment, we used the redundant target paradigm together with CFS, by bilaterally presenting fearful and angry bodies, and suppressing one of the visual fields to emulate a blind field. Because we previously observed a longer suppression time under CFS for fearful bodies, and a shorter suppression time for angry bodies (Zhan, Hortensius, \& de Gelder, 2015), we chose neutral, fearful and angry bodies in the second and the third experiment.

\section{General method}

The materials and methods similar across all 3 experiments were described here. Detailed settings that differed across experiments were described in individual sessions.

\section{Participants}

The participants were recruited from Maastricht University. All participants except one had normal stereo and color vision, and normal or corrected-to-normal visual acuity. None of the participant had a history of neurological disorders. The participants provided written consents, and received either monetary or course credit rewards after participation. The experimental procedures were approved by the ethical committee of Maastricht University, and the experiments were carried out in accordance to the declaration of Helsinki.

\section{Stimuli}

The stimuli of 3 experiments were all embedded in a gray rectangle of $160 \times 240$ pixels (RGB value $=128,128,128)$. Static gray-scale face and body images were used. The face images were adapted from the Radboud Face database (Langner et al., 2010). All faces were aligned at the eye level, and placed with their tip of noses at the center of the rectangles. An ellipse 


\section{Chapter 3}

mask was placed on the faces, so that visual details outside the ellipse were masked out. The faces occupied a region within $75 \times 102$ pixels.

The body images were adapted from Stienen and de Gelder (2011), with facial information removed, aligned at the feet level, and were all positioned at the center of the rectangle. The bodies occupied a region within $131 \times 193$ pixels.

\section{The dichoptic display}

The experiments were presented with Psychtoolbox 3 (Brainard, 1997; Pelli, 1997) in MATLAB (version 2012b, the MathWorks, Natick, MA, USA). For stable refresh rates and precise timing of the stimuli presentation, the experiments were presented with a $3 \mathrm{D}$ capable LCD screen (Acer VG248, resolution $=1920 \times 1080$, refresh rate $=60 \mathrm{~Hz}$ ). The dichoptic display of stimuli was achieved by presenting two stimuli side-by-side with each other at the center of the screen, while the participants viewed the screen through a pair of prism glasses, which bent the light from the screen and projected the ipsilateral image to the center of the view of each eye (diopters of the prism glasses: 7 for Experiment 1, 8 for Experiment 2, 12 for Experiment 3). The background of the screen was set to gray (RGB value $=128,128,128$ ). To aid fusing of the stimuli for participants, two rectangular frames (thickness $=10$ pixels) were placed around the stimuli, and two fixation crosses were placed at the center of each rectangle. To prevent crosstalk of the two images, a cardboard was placed between the screen and the participant, which separated the view of the screen in two equal halves. Participants rested their heads on a chin rest $59 \mathrm{~cm}$ from the screen. Under this setup, when the participants achieved a stable fusion of the two rectangles, they would see one single rectangle, and one single fixation cross at the center of the screen. To indicate the start of each trial, the fixation cross would change to white color $1 \mathrm{~s}$ before the trail, and kept white throughout the trial. Participants were instructed to keep their heads as still as possible, remain fixating well on the fixation cross, and not to blink within a trial if possible.

For the CFS presentation, one target stimulus was presented on one rectangle, while dynamic and colorful noise patterns (flash rate $=10 \mathrm{~Hz}$ ) were presented in the other. Each noise pattern consisted of overlapping small rectangles of different colors (height and width within 20x15 pixels). Six hundred unique noise images were created, and were randomly selected for each individual trial.

To decrease the possibility that the target stimulus escape suppression, the target stimulus was faded in from $0 \%$ to a fixed contrast ( $30 \%$ for experiment $1,50 \%$ for experiment 2 and 3 ), kept at the contrast for a time period ( $1 \mathrm{~s}$ for experiments 1 and 2, $0 \mathrm{~s}$ for experiment 3 ) and then faded out to $0 \%$ contrast. The flashing noise patterns were kept on the screen for a few more seconds after the target stimulus disappear, to prevent the target stimulus creating an afterimage. The timings of the fading in and out were reported separately under each experiment. 


\section{Affective Priming}

\section{Procedure}

In the experiment, participants were asked to rate their likability for a number of Chinese characters, preceded by suppressed prime stimuli under CFS. The participants were not familiar with Chinese characters or Kanji. The experiment consisted of 2 blocks, one for face primes, and one for body primes. The orders of the blocks were randomized across participants. In each block, 4 types of prime stimuli were presented: blank (gray background, RGB $=128,128,128$, no prime), polygons, fearful stimuli, happy stimuli. Each stimulus type had 24 trials per block, resulting in a total of 96 trials per block. The fearful and happy stimuli were matched for identities ( 24 actors for face and body stimuli respectively). Twenty-four polygon stimuli were made by combining 10 cubes in SketchUp (https://www.sketchup.com/), and rotated in different angles. A total of 72 Chinese characters were selected from the Table of General Standard Chinese Characters (Ministry of Education of the People's Republic of China, http://www.gov.cn/gzdt/att/att/site1/20130819/tygfhzb.pdf). They were 5-stroke characters with high frequency. Symmetrical characters were avoided to prevent participants rating them with higher likabilities. All characters were rendered in the Imitation Song typeface, and occupied a region of $138 \times 147$ pixels. For each block, the 72 characters were randomly split into 3 sets ( 24 characters each), one set was presented after both fearful and happy primes; the other two sets were presented after the blank and the polygon primes respectively. See Figure 1 for stimuli examples.

For each trial, the prime stimulus was presented randomly in one of the eyes, by fading it in from 0 to $30 \%$ contrast for $0.5 \mathrm{~s}$, staying at $30 \%$ contrast for $1 \mathrm{~s}$, and fading out for $0.5 \mathrm{~s}$. The noise patterns were presented in the other eye from the onset of the trial, and was flashed 2 more seconds after the fading out of the prime stimulus. The Chinese character was presented after the disappearance of the noise patterns for $2 \mathrm{~s}$. The participants rated their likability of the character within the $2 \mathrm{~s}$ by pressing one of 4 buttons with their left hand, with 1 being "do not like it at all", and 4 being "like it a lot". The next trial started after a $4 \mathrm{~s}$ interval. Before the two blocks of the experiment, participants did a 10-trial practice block with different characters and with only the blank stimulus as prime. 


\section{Chapter 3}

A Dichoptic presentation
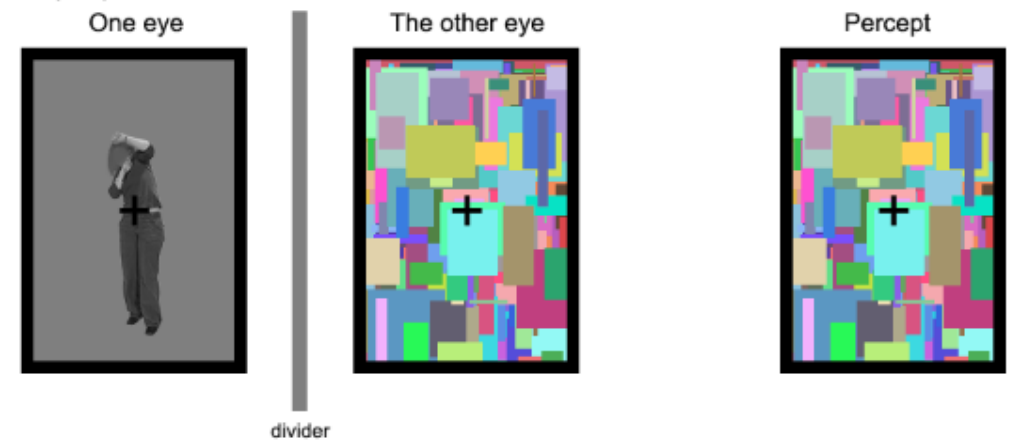

B Prime stimuli
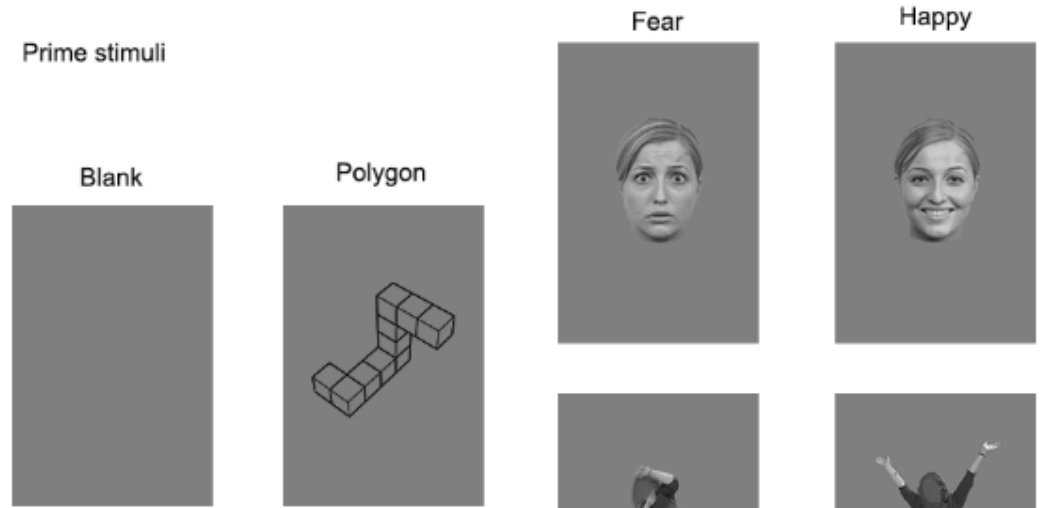

Target stimuli
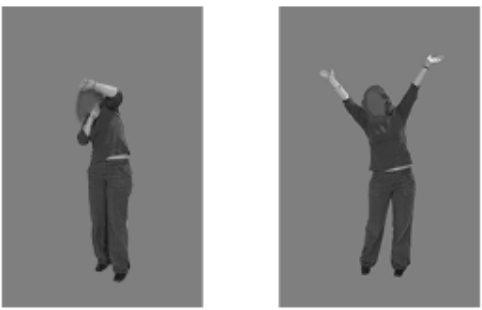

Character

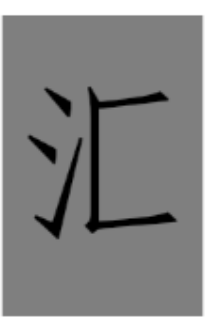

Figure 1. Example stimuli of experiment 1. A. The dichoptic presentation, and the percept of the participant. B. Examples of prime and target stimuli.

Forty-four participants (age range 19-35) took part in this version of experiment. Participants were debriefed after the experiment, for whether they saw anything in the noise pattern, and their impression of the characters. Participants reported in general that they found the characters preferable. To avoid a ceiling effect of rating the characters too high, 20 of the participants were asked to rate 225 Chinese characters ( 5 strokes and 6 strokes, other selection criteria and the rendering was the same as the ones in the first version) in a separate experimental block. The stimulus size and the procedure was the same to the main experiment, only without the CFS priming part, and wasn't a dichoptic presentation. A onesample $t$ test against 2.5 (the midpoint of the 4-point scale) was performed for each character, and those characters with $p<0.05$ were excluded. In this way 72 characters were selected for a second version of the experiment, and were rendered in the Song typeface, which was less similar to the style used in Chinese calligraphy. The second version of the experiment was carried out with the modified characters, while keeping the other settings the same to the first version. 
19 participants took part in the second version (age range 19-33). The datasets of participants who reported seeing bodies or polygons were excluded, and for those who didn't see bodies but saw faces, the data were excluded for the dataset of face primes.

For the first version, 26 out of the 44 participants had percepts suppressed for bodies ( 3 males, mean age $=21.9$ ), and within them only 14 whose percepts were also suppressed for faces. For the second version, only 8 participants had percepts suppressed for bodies ( 2 males, mean age $=23.8$ ), within them only 3 whose percepts were also suppressed for faces. Another participant's percept was suppressed for faces but not for bodies. Due to the small amount of usable datasets in the second version, only the data for the first version were analyzed. Trials without responses were excluded from the analysis. For the body-prime dataset, $3.21 \%$ of the trials were excluded; for the face-prime dataset, $7.37 \%$ of the trials were excluded.

We analyzed the subjective ratings and the RTs separately. We initially z-normalized the ratings within each participant, following Almeida et al. (2013). Since the z-normalized facehappy, body-polygon, body-fear conditions did not have a normal distribution (Shapiro-Wilk test, $p=.042, p=.0001, p=.047$ respectively), we used the non-normalized data for the following analyses (all had normal distribution). For the participants with percepts suppressed for bodies $(n=26)$, the 4 stimulus types were entered into a repeated measures ANOVA; for the participants that percepts were both suppressed for faces and bodies $(n=14)$, all conditions were entered into a face/body $x$ stimulus type (4 types) repeated measures ANOVA. The effect of the order that participants performed the face and body blocks was analyzed by assigning the order as a between-subject factor in the ANOVA.

\section{Results and discussion}

For the data of the body-prime block, the ratings of Chinese characters primed by the 4 stimuli types did not differ with each other, $F(3,75)=0.733, p=.535, \eta_{p}{ }^{2}=.029$. The RT of the 4 stimuli types did not differ either, $F(3,75)=0.901, p=.445, \eta_{p}{ }^{2}=.035$. The order that participants did the face and body blocks did not influence the results (between-subjects effect of order was not significant, for ratings: $F(1,24)=0.096, p=.760, \eta_{p}{ }^{2}=.004$; for RT: $\left.F(1,24)=2.916, p=.101, \eta_{p}^{2}=.108\right)$.

For the participants with percepts suppressed for both bodies and faces, the ratings of Chinese characters and the RTs were not different between the body and face datasets, for ratings: $F(1,13)=0.007, p=.934, \eta_{p}{ }^{2}=.001$, for $R T s: F(1,13)=0.226, p=.642, \eta_{p}{ }^{2}=.017$. No difference was found among the 4 different prime stimulus types, for ratings: $F(3,39)=1.185$, $p=.328, \eta_{p}{ }^{2}=.084$, for RTs: $F(3,39)=0.812, p=.495, \eta_{p}{ }^{2}=.059$. No interaction was observed between the face/body datasets and the stimulus types, for ratings: $F(3,39)=0.069, p=.976$, $\eta_{p}{ }^{2}=.005$, for RTs: $F(3,39)=0.432, p=.731, \eta_{p}{ }^{2}=.032$. The order that participants did the face and body blocks did not affect the ratings or the RT (between-subjects effect of order was not significant, for ratings: $F(1,12)=2.999, p=.109, \eta_{p}{ }^{2}=.200$; for $R T s: F(1,12)=0.390, p=.544$, $\left.\eta_{p}^{2}=.031\right)$. 


\section{Chapter 3}

Although both the averaged ratings primed by fearful faces and bodies were lower than the ones primed by blank stimuli, these differences were not significant ( $p>$.05). See Figure 2 .

The individual data showed inconsistent patterns (data not shown).

The averaged ratings for Chinese characters across conditions were not significantly different from neutral (one-sample t tests against 2.5, neutral), for the face-primed data: mean $=2.643, t(13)=1.550, p=.145$; for the body-primed data: mean $=2.611, t(25)=1.782$, $\mathrm{p}=.087$.
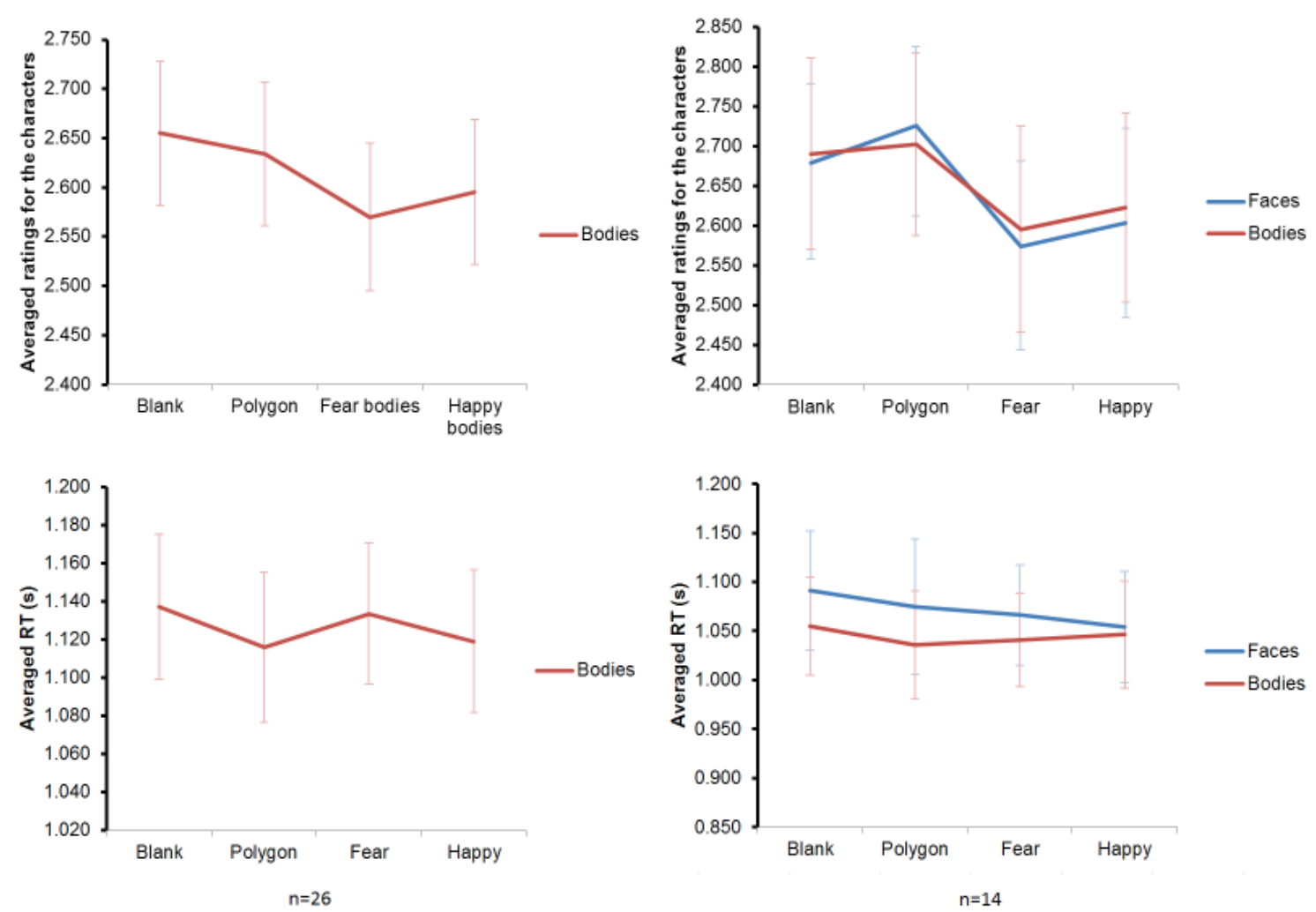

Figure 2. Averaged ratings for the Chinese characters, and the RTs, for the body dataset $(n=26)$ and the body + face dataset $(n=14)$. Error bars denote SEM.

While Almeida et al. found a negative priming effect with angry faces under CFS (Almeida et al., 2013), we did not observe a priming effect for either faces or bodies, with either fearful or happy emotion. It may be that we did not test angry bodies here, and fearful bodies may not exhibit a strong priming effect. Although fearful faces were previously found to be processed by the amygdala under CFS (Jiang \& He, 2006), here due to a low number of participants whose percepts of faces were suppressed, we could not detect a reliable behavioral priming effect for fearful faces either. We had to exclude a large number of participants (18 out of 44 in experiment version 1 ) due to the prime stimuli breaking from suppression, this was also the case for Almeida et al. (2013) where they excluded 26 of 54 participants. This indicates that the CF-suppression strength for a stimulus type at a fixed contrast has large inter-individual variability.

Apart from the variable CF-suppression strength that resulted in participant data exclusion, we observed inconsistent patterns of the 4 conditions across individual participants included in data analysis, which again indicate large inter-individual variability, but either in the 
conscious aesthetic perception of Chinese characters, or in the unconscious priming effect. Indeed, a recent $\mathrm{fMRI}$ experiment with the affective priming paradigm under CFS showed such evidence. In that study, participants were primed with either fearful faces or flowers, and rated the likability of novel neutral faces. The study found that the negative bias across participants induced by suppressed fearful faces was associated with higher amygdala activation (Lapate et al., 2016).

Another reason may be that, the suppression of CFS we used was too strong to induce a behavioral effect. Almeida et al. used only two flash frames of $100 \mathrm{~ms}$ each (Almeida et al., 2013), while we had $2 \mathrm{~s}$ of priming plus $2 \mathrm{~s}$ dynamic noise patterns after priming to avoid afterimages. It has been shown in a previous study that the strength of CFS builds up by consecutive frames of flashes: 5 consecutive flashes had the strength comparable to the normal CFS, while 1-4 flashes had significantly weaker suppression strength (Tsuchiya et al., 2006). Another study (Faivre et al.) using long priming periods under CFS ( $2500 \mathrm{~ms}$ ) compared priming effects of happy and angry faces on Chinese characters, but did not find a preference bias for either emotion. Although in their study when characters were replaced by faces of the same emotion, either identical to or have a different identity to the primes, participants showed a repetition priming effect, indicating that the suppressed primes were processed to some extent (Faivre, Berthet, \& Kouider, 2012). Thus either our CFS procedure did not allow the priming effect to transpire, or the priming effect may have disappeared during the $2 \mathrm{~s}$ of noise after the priming period.

\section{Body detection}

\section{Procedure}

In each trial of this experiment, a target image was presented randomly to one of the eyes of the participant. The target image was either a blank stimulus with gray background, or a bodily action (neutral: calling on the phone, or angry) performed by 24 actors ( 12 females). The dynamic noise patterns were at the same time presented into the other eye of the participant. The target stimulus was faded in for $0.5 \mathrm{~s}$, kept at $50 \%$ contrast for $1 \mathrm{~s}$, faded out in $0.5 \mathrm{~s}$, with the dynamic noise flashing for 2 more seconds. The participants indicated the visibility of the target stimulus, and their confidence of response, by pressing keyboard button 1 or 2 per trial at their own pace (Q1: Seen a body? Yes/No; Q2: Sure/Guessed). The next trial started after a 3 s inter-stimulus interval after the participant's response for Q2.

The bodies were presented in 8 blocks; each containing 12 bodies from one same category (either neutral or angry) and 12 blank trials. The order of the individual bodies and the order of the neutral and angry blocks were fully randomized.

After the experiment, the participants also filled in the State trait anxiety inventory (STAI) and the Interpersonal reactivity index (IRI) questionnaires. The total STAI scale and the 4 scales of the IRI (PT: perspective-taking; FS: fantasy; EC: empathic concern; PD: personal distress) were scored for each participant.

Thirty-eight participants took part in the experiment. Within them, 2 participants' data were not logged, and 1 other participant wasn't able to maintain fusion during the experiment. So 


\section{Chapter 3}

their data were excluded from analysis. The data of the remaining 35 participants (9 males, age range $18-31$, average age $=22.7$ ) were used in the analysis.

The $d^{\prime}$ and the confidence of ratings for the neutral and angry bodies were calculated separately, following the calculation in (Stienen \& de Gelder, 2011). The d' was calculated by the following formula:

$d^{\prime}=z\left(H^{\prime}\right)-z\left(F A^{\prime}\right)$

$H^{\prime}=(h+0.5) /(h+m+1)$

$F A^{\prime}=(f+0.5) /(f+c r+1)$

Where $h=$ number of hits, $m=$ number of misses, $f=$ number of false alarms, $c r=$ number of correct rejections. $\mathrm{z}$ is the inverse of the normal distribution. The $\mathrm{H}^{\prime}$ and $\mathrm{FA}^{\prime}$ were computed with adjustments to avoid getting $z$ values of infinity when hit rate or false alarm values were at 1 or 0 (Snodgrass \& Corwin, 1988).

The confidence was calculated by: $(\mathrm{ST}(\mathrm{h}+\mathrm{cr})-\mathrm{ST}(\mathrm{m}+\mathrm{f}))$ / total number of trials, where $\mathrm{ST}$ is the number of trials that participants reported "sure".

We examined whether the $d^{\prime}$ and confidence scores were significantly different between the neutral and angry conditions. Since the $d^{\prime}$ and confidence for both neutral and angry bodies violated the normality assumption (Shapiro-Wilk test of normality, all $p<0.05$ ), the difference scores of $d^{\prime}$ and confidence between neutral and angry bodies were calculated (BA-BN_d', BA-BN_confidence, the confidence difference score again violated the normality assumption, Shapiro-Wilk test of normality, $\mathrm{p}=.018$ ), and underwent the Wilcoxon signed rank test against a median of 0 . To also see whether the difference of perception between the two actions were reflected in the questionnaires, both the correlation between these two difference scores and their correlations to the 5 questionnaire scales were investigated by Spearman's rank correlation across participants.

\section{Results and discussion}

The $d^{\prime}$ and confidence values for neutral and angry bodies were relatively low. Neutral bodies: mean $\mathrm{d}^{\prime}=0.770, \mathrm{SD}=0.901$; mean confidence $=0.027, \mathrm{SD}=0.270$. Angry bodies: mean $d^{\prime}=0.826, S D=1.011$; mean confidence $=0.215, S D=0.280$. For the neutral and angry conditions, each had 7 participants with $d^{\prime}$ values of 0 ( 3 of them had $d^{\prime}=0$ for both conditions).

Between the angry and neutral bodies, none of the difference scores for either $d^{\prime}$ or confidence were significantly different from a median of 0 ( $p=.704, p=.703$ respectively), which did not show advantage of angry bodies over neutral ones on detection under CFS.

The difference scores of $d^{\prime}$ and confidence were correlated between each other across participants (Spearman's rho $=.688, p<.001$ ), but they were not correlated to any of the questionnaire scales (all $p>.37$ ).

Our findings are consistent with the finding of Stienen and de Gelder (2011), where the d' and confidence ratings co-varied for angry bodies. However, we did not find a dissociation of 
performance with confidence rating, for either angry or neutral bodies. Thus it did not explain our previous finding, why the angry bodies were suppressed shorter under CFS (Zhan et al., 2015), see Chapter 2. Because our measure of $d^{\prime}$ and confidence were low, the lack of dissociation may indicate that the suppression of awareness under CFS is too deep, that we had a floor effect.

\section{Bilateral presentation of body stimuli}

\section{Stimuli}

The stimuli consisted of 6 fearful and 6 angry postures, performed by 6 actors ( 3 males). Two rectangular frames of $320 \times 240$ pixels were placed side by side, each with a fixation cross in the rectangle center. With the dichoptic presentation, each eye of the participant saw the content within one rectangle, and the participant's left and right visual fields corresponded to the left and right side of the fixation cross for both eyes. In each trial, the same target stimulus was presented in one visual field of both rectangles. In the other visual field of one rectangle, either no stimulus (blank, baseline conditions), or a stimulus with congruent or incongruent emotion was presented. In the corresponding visual field of the other rectangle, a series of dynamic noise patterns were presented. The noise patterns suppressed the perception of the other stimulus in the corresponding visual field, which rendered a percept of only one stimulus presented side by side with the flashing noise patterns. There were 6 conditions of suppressed stimuli in a total of 192 trials: fear-congruent, fear-incongruent, fear-blank, anger-congruent, anger-incongruent, anger-blank. To balance the number of times that a certain stimulus was seen, the congruent and incongruent conditions had 24 trials each, and the blank conditions had 48 trials each. For the same reason, the noise pattern was presented in the right eye for congruent and incongruent trials, and was presented in the left eyes for blank trials. The visual field that the noise pattern was project into was balanced across trials, and later served as a factor in the analysis. See Figure $\mathbf{3}$ for stimuli and conditions.

For the conditions where two bodies were presented in the same rectangle, the identities of the bodies were always different from each other, but both were from the same gender.

In each trial, the target stimulus was faded in for $0.5 \mathrm{~s}$ to $50 \%$ contrast, and then faded out $0.5 \mathrm{~s}$ to $0 \%$ contrast. Participants performed an 2AFC task, where they reported the emotion they saw in the non-noise side as quickly but as accurately as possible during stimulus presentation, by pressing one of the two buttons (numpad button 1 and 2). If the participant didn't make a response, the noise patterns would continue to flash for 2 more seconds after the target stimulus faded out. Thus the response window was in total $3 \mathrm{~s}$. To eliminate the possibility of seeing afterimages in the suppressed visual field, the noise pattern would be presented in both eyes for 1 more second immediately following the participant's response, or after the response window closed. The inter-stimulus interval was jittered among 3.5, 4, $4.5,5,5.5$ seconds. 


\section{Chapter 3}

A

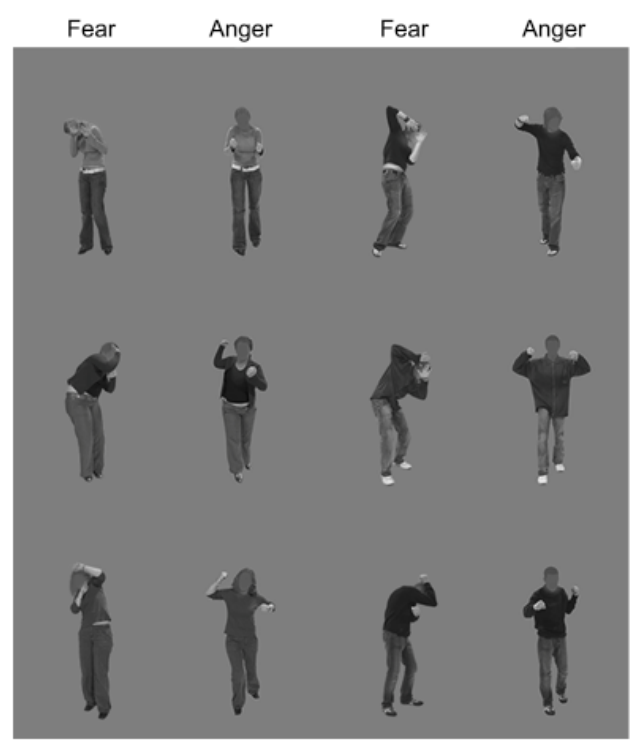

B
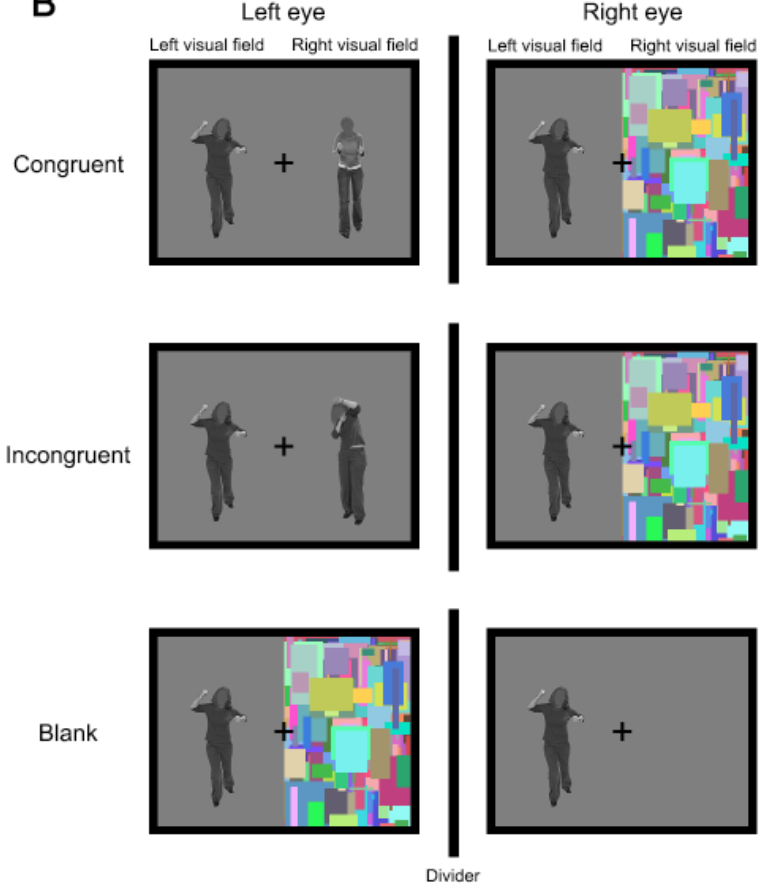

C

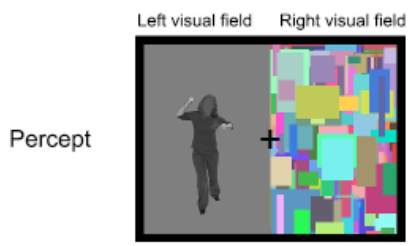

Figure 3. A. All body stimuli used in this experiment, performed by 6 actors. B and C. Stimuli presentation, with examples showing an angry body in the left visual field as the target, while the noise pattern suppressing stimuli in the right visual field. B. Conditions of angry-congruent, angry-incongruent, and angry blank. C. The 3 conditions in B all resulted in the same percept. The fearful conditions were constructed in the same way. In the experiment the visual field that the noise patterns were projected into for each trial was randomized. This figure shows right-visualfield projection of noise patterns.

\section{Procedure}

Before the actual experiment, participants underwent a short practice of 12 trials, where fearful and angry bodies different from the actual experiment were presented unilaterally (the side suppressed by the noise pattern was blank).

In the actual experiment, the button assignments corresponding to the fear and anger responses were balanced across participants.

Forty-one participants took part in the study. For each participant, we included the data for further analysis if they satisfied two criteria: 1 . the participant performed the task properly (low number of incorrect trials); 2 . the CFS suppressed the stimuli properly throughout the experiment, in which case the participant should not consciously perceive the suppressed stimulus in the noise at all. This was validated by a series of debriefing question after the experiment (Did you see anything in the noise? If yes, how many times? Did you see two boxes during the experiment? If yes, how many times?). In studies of perceiving non- 
conscious stimuli, a trial-by-trial report of visibility has been usually implemented, which could be a dichotomous report (seen/unseen), or a graded report (e.g. perception awareness scale, PAS, Rams $\varnothing$ y \& Overgaard, 2004). For normal participants, hemianopia and blindsight patients, PAS has been a more sensitive measure of visual awareness than yes/no answers (Mazzi, Bagattini, \& Savazzi, 2016), reflected the level of priming effects (Lohse \& Overgaard, 2017), and correlated with the ERP amplitude of the visual awareness negativity (Mazzi, Tagliabue, Mazzeo, \& Savazzi, 2018). In the current study that directed participants' attention to one of the visual fields, we did not implement a trial-by-trial measure of awareness. Apart from maintaining a design comparable to other redundant-target studies, we are aware of the possibility, that if participants report their subjective awareness on a trial-by-trial basis, they may expect to see stimuli in the noise, which may direct their attention to the noise side and in turn interfere with the emotion categorization task they perform. Consequently we chose a stringent criterion, and included participants who were completely unaware of the suppressed stimuli.

The following participants' data were excluded from the analyses: one participant had a lazy eye; two participants saw the stimuli in the noise; another two participants did not see any suppressed stimuli but saw two boxes once and twice respectively during the experiment, indicating imperfect merging; another participant had only 5 trials of correct responses. In total 35 participants' data were included in subsequent analysis (participants' mean age $=21.97$, range 18-27 years, 4 males, 3 left-handed). These participants were not aware of the presence of suppressed stimuli. The numbers of correct trials per condition were counted. Since the total number of trials for blank conditions and for the congruent/incongruent conditions were not the same, and the trial counts in most of the conditions were not normally distributed, two Friedman tests were performed respectively for the 4 blank conditions (fear/happy, L/R visual field that the target was projected into), and for the 8 congruent/incongruent conditions.

The average RT for each condition of suppressed stimuli was calculated for each visual field of each participant (LVF, RVF). For this analysis, independent of the correctness of the responses, the trials that had an RT outside 1.96 standard deviations of the average RT within each participant were first excluded ( $4.91 \%$ of all trials). Trials not responded $(0.36 \%$ of all trials) and trials with a wrong response (5.22\%) were also excluded. This rendered a total of $10.49 \%$ trials excluded. The 12 conditions were then entered into a repeatedmeasures ANOVA of factors conscious emotion, congruency (blank/congruent/incongruent), and visual field (L/R). We also performed an ANOVA by using the same inputs but coding the conditions differently, with factors of conscious emotion, unconscious emotion (blank/fear/anger), and visual field. To examine the whole distribution of the RTs for individual participants, which provides more information comparing to using only the mean RT per participant, we further computed the cumulative distribution functions (CDF) for each condition (Ratcliff, 1979). Because no visual field laterality effect was found in the two ANOVAs (see results), we first rank-ordered the RT in individual participants for the 6 conditions (conscious emotion: fear/anger, congruency: blank/congruent/incongruent) across both visual fields, divided the RTs in $10 \%$ quantiles, then calculated the mean for each quantile. This procedure was performed with the excel plugin CDF-XL (Houghton \& Grange, 


\section{Chapter 3}

2011). These RTs were then entered into a repeated-measures ANOVA of conscious emotion $x$ congruency $\times$ quantiles.

For all the ANOVAs performed, when the sphericity is violated, Greenhouse-Geisser corrections were applied. Multiple comparisons were adjusted with the Sidak method in post-hoc simple effect analysis.

In addition, we also checked whether a Simon effect was present in the data, between the situations that the side of the button assignment was congruent with the stimulus presentation side, versus those incongruent ones (e.g. if the participant was assigned the buttons of $1=$ fear and $2=a n g e r$, a trial where fear was presented at the left visual field would be a congruent trial). The Simon effect on accuracy showed a trend to significance in the two-sided Wilcoxon signed ranks test, $\mathrm{Z}=-1.928, \mathrm{p}=.054$, where the accuracy for the congruent side was slightly higher. In the ANOVA conscious emotion (fear/anger) * congruency (blank/congruent/incongruent) * button congruency (congruent/incongruent), the congruent button condition showed faster RTs with a trend to significance (mean difference $\left.=0.010 \mathrm{~s}, F(1,34)=4.083, p=.051, \eta_{p}^{2}=.107\right)$, but did not have interactions to the other two main effects $(F(1,34)=0.617, F(1,34)=0.022$ respectively), thus the Simon effect was not included as a factor in the main analysis.

\section{Results}

The numbers of correct trials did not differ among the 4 blank conditions (Fblank_Lfield, Fblank_Rfield, Ablank_Lfield, Ablank_Rfield), $\chi^{2}(3)=3.137, p=.371$. The numbers of correct trials among 8 congruent and incongruent conditions were not different either, $\chi^{2}(7)=2.294$, $\mathrm{p}=.942$.

The repeated measures ANOVA (conscious emotion $\times$ congruency $\times$ visual fields) for the RTs showed a main effect of congruency, $F(2,68)=9.535, p=.00022, \eta_{p}{ }^{2}=.219$, where the incongruent conditions had a shorter $\mathrm{RT}$ than both the blank and the congruent ones (RT mean difference $=0.014 \mathrm{~s}, \mathrm{p}=.020$, and $\mathrm{RT}$ mean difference $=0.020 \mathrm{~s}, \mathrm{p}=.00081$, respectively). Examining fear and anger separately with post-hoc simple effect analysis, the RT of conscious angry body together with an unconscious incongruent fearful body was significantly shorter than with a congruent angry body (mean difference $=0.031 \mathrm{~s}, \mathrm{p}=.00074$ ), and also shorter than presenting the conscious angry body alone (mean difference $=0.021 \mathrm{~s}$, $p=.011$ ). See Figure 4 .

There was also a trend to significance for the interaction of conscious emotion $\times$ visual fields, $F(1,34)=3.956, p=.055, \eta_{p}^{2}=.104$. Post-hoc simple effects analysis showed that, in the right visual field, the conscious angry bodies across 3 congruency conditions in general had a longer RT than the conscious fearful bodies (RT mean difference $=0.019 \mathrm{~s}, \mathrm{p}=.023$ ).

None of the other main effects or interactions was significant.

In the ANOVA of conscious emotion $\times$ unconscious emotion $\times$ visual fields, where the conditions were coded differently, we found a significant interaction of conscious and unconscious emotions, $F(2,68)=8.982, p=.00035, \eta_{p}{ }^{2}=.209$. There was a trend to significance for conscious emotion $\times$ visual field, $F(1,34)=3.956, p=.055, \eta_{p}^{2}=.104$, the same to the 
ANOVA above. There was also a trend to significance for unconscious emotion, $F(2,68)=2.863, p=.064, \eta_{p}{ }^{2}=.078$, where the unconscious fearful bodies had a slightly shorter $\mathrm{RT}$, although pairwise comparisons were not significant.

To further examine the whole distribution of the RTs in individual participants, we calculated the means of rank-ordered RTs in 10\% quantiles, and performed an ANOVA of conscious emotion $\times$ congruency $\times$ quantiles. There was a significant main effect of congruency $\left(F(2,68)=13.277, p=.0000135, \eta_{p}{ }^{2}=.281\right)$, that the incongruent conditions had the shortest RT ( $p=.002$ versus congruent conditions, $p=.00026$ versus blank conditions), consistent with the ANOVA results calculated with condition means. There was a trend to significance for the main effect of conscious emotion, that the RT for conscious fear was marginally shorter than that of conscious anger $\left(F(1,34)=3.574, p=.067, \eta_{p}{ }^{2}=.095\right)$. The main effect of quantiles was significant, due to the rank-ordering process. Interestingly, there is an interaction of congruency $\times$ quantiles $\left(F(2.819,95.845)=5.696, p=.002, \eta_{p}{ }^{2}=.143\right)$, that the difference of RT for incongruent conditions was bigger for the longer RTs, comparing to the blank and congruent conditions.
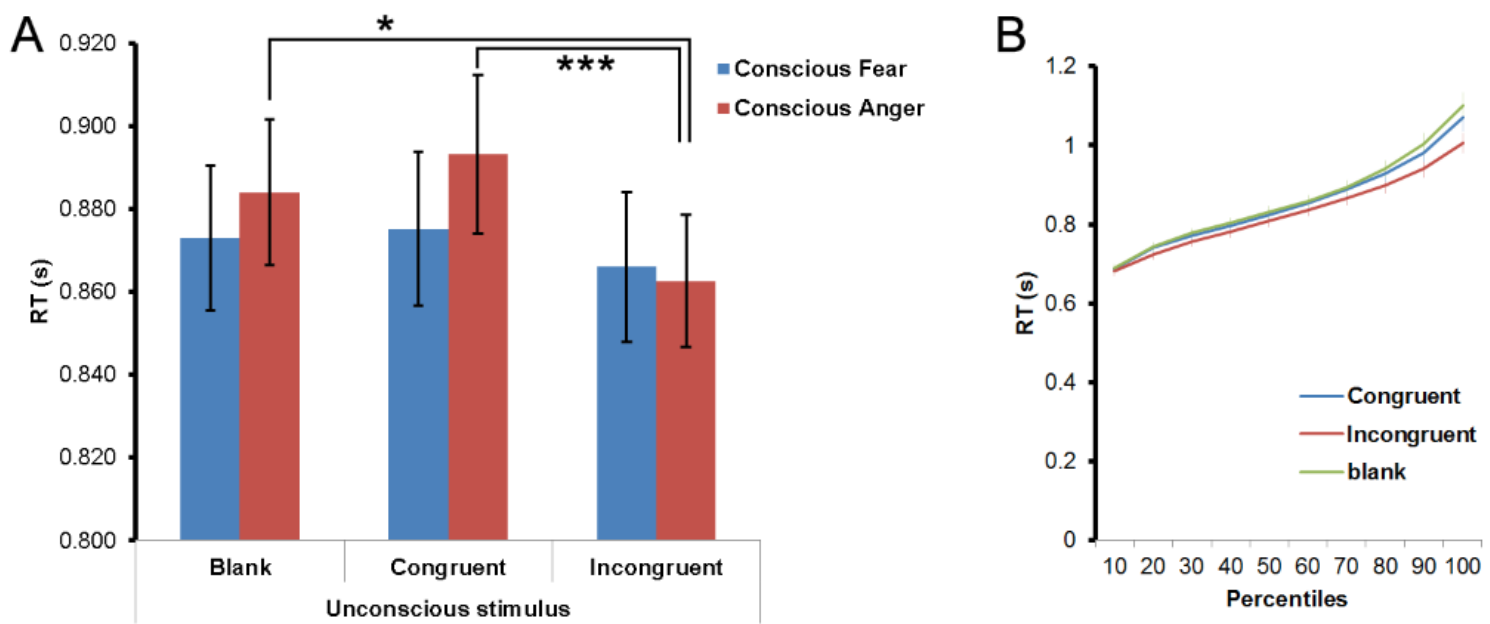

Figure 4. A. Reaction times (RTs) of conscious fear and anger, presented together with an unconscious stimulus, which was a blank stimulus, a congruent stimulus, or an incongruent stimulus $(n=35)$. Error bars denote SEM. $*: p<.05,{ }^{* * *}: p<.001$. B. Cumulative distribution functions of rank-ordered RTs, averaged for congruent, incongruent and blank conditions ( $n=35)$. Error bars denote SEM.

\section{Discussion}

Our goal was to find evidence for perception of emotional body expressions outside visual awareness in healthy participants by using the redundant target effect and the CFS paradigm, and to compare to patient studies. We found an interaction between the body expressions of the consciously and the unconsciously seen stimuli. We expected that here the RT would typically be shorter when comparing the congruent condition to the single-stimulus condition, in line with the traditional redundant target effect observed with stimuli under 


\section{Chapter 3}

both fully visible conditions and backward-masked conditions. However, we did not find a facilitation effect by the congruent conditions, but instead found a faster RT for the incongruent conditions. This indicates that the unconscious process during CFS measured here may be sustained by different mechanisms at stake in backward masking, and under conditions of full visibility. Under CFS, the unconscious bodies were processed to some extent, but the processing was not at a level that would allow a redundant target effect to appear, unlike the findings for healthy participants with conscious perception or under masking conditions, and for blindsight patients. However we found that the incongruent condition showed a bigger RT difference than congruent and blank conditions. This may reflect that the information of the suppressed incongruent stimuliaccumulates over time, that some information was indeed processed, despite the strong suppression effect of CFS.

The congruency effect we found appears to have a level of emotion specificity: it was more strongly shown when the consciously perceived bodies were angry ones. Unconscious fearful bodies facilitated the conscious perception of angry bodies, but unconscious angry bodies did not. Previously we found that angry bodies were suppressed for a shorter time span than the fearful bodies (Zhan et al., 2015), which may in part explain the differential effects between the unconscious emotions observed here: the unconscious angry bodies may be more salient and therefore may act like distractors, thus slowing down the RT comparing to unconscious fearful bodies.

Compared to patient studies, the facilitation effect of unconscious fearful bodies was previously found in neglect patients, where the fearful bodies in the contra-lesional side were extinguished less (Tamietto et al., 2007). However, this attention-grabbing effect of fearful bodies would indicate a slower RT for the consciously perceived emotion in our study, which we did not find. Also, neglect is thought to be induced by a disruption of balance in the spatial and non-spatial attentional controlling systems (Corbetta \& Shulman, 2011). In comparison, the CFS paradigm itself did not require participants to switch attention to another location, or interfere with this process. In our experimental design the switch of attention was explicitly driven by task instructions in each block. Thus, it seems that the CFS disrupts the conscious percept by a different mechanism than neglect.

On the other hand, our results showed similarities to the hemianopia patients without exhibiting blindsight effects. These studies found a facilitation effect for unconscious fearful faces, on both emotional and non-emotion-related tasks (Bertini et al., 2013, 2017). In one of the studies, the fearful faces presented to the blind visual field did not show a facilitation effect when the intact field also perceived a conscious fearful face (Bertini et al., 2013), which was similar to what we found for congruent fearful bodies. The authors postulated that the conscious presentation of fearful faces may have inhibited the unconscious processing of fear through subcortical routes (Bertini et al., 2013). Future fMRI studies are necessary to validate this assumption. Interestingly, a follow-up study applied inhibitory transcranial direct current stimulation (tDCS) on either the vertex or the left occipital cortex (corresponding to $\mathrm{O} 1$ of the EEG 10/20 system) of healthy participants, both controlled with sham stimulations. Participants performed a go/no-go task to bilaterally presented emotional faces (fear, happy), masked by a neutral face. The study found that both under the sham condition and when inhibiting the vertex, the RTs for happy and fearful target 
faces were facilitated when the masked face had congruent emotions, a redundant target effect similar to those found in other masking and blindsight studies. However, inhibiting the occipital cortex showed a facilitation effect of masked fearful faces on target happy faces, similar to hemianopia patients without blindsight (Cecere, Bertini, \& Ladavas, 2013). Given that the interocular competition utilized by the CFS paradigm is thought to occur in V1 or LGN (Tong et al., 2006), this tDCS study presented particularly intriguing similarities to CFS.

Although the previous hemianopia studies and our study showed a facilitation effect for unconscious fear, no matter whether it was expressed by faces or bodies, it is still not fully clear whether both stimuli categories convey emotional information through similar neural substrates, or whether these neural substrates differ across different groups of participants. The faces convey fear with raised eyebrows and increased eye-white regions (Whalen et al., 2004), which in both sighted participants and blindsight patients rely on a fast subcortical pathway, that relay both broadband and low-spatial-frequency information, through the superior colliculus, inferior pulvinar, to amygdala (Burra, Hervais-Adelman, Celeghin, de Gelder, \& Pegna, 2017; Mendez-Bertolo et al., 2016). The importance of intact superior colliculi for blindsight is supported by an RTE effect for gestalt-like dots in blindsight patients with hemispherectomy, for whose blind visual fields, no other intact structure in the visual pathway existed apart from the superior colliculus (Georgy, Celeghin, Marzi, Tamietto, \& Ptito, 2016). However the involvement of the amygdala for faces may be linked to the eye region of the faces, in both masking and CFS experiments (Gray, Adams, Hedger, Newton, \& Garner, 2013; Whalen et al., 2004; Yang et al., 2007). Comparing to the faces, perception of bodily emotions involves more action-related processing (Dael, Mortillaro, \& Scherer, 2012; de Gelder et al., 2010). Although extinguished fearful bodies in a neglect patient could activate the amygdala (Tamietto et al., 2015), the preferred spatial frequency conveying emotional information for bodies is yet to be determined. The processing of fearful faces and bodies are also likely to be different under CFS, as we previously found a shorter suppression time for fearful faces than angry ones, but a longer suppression time for fearful bodies than angry ones (Zhan et al., 2015). Given the facilitation effect of fear (bodies/faces) in our findings and in hemianopia patients without blindsight, the RTE difference we found to blindsight may be more related to the individual processing mechanisms (e.g. stages in different pathways) disrupted by these conditions, rather than stemming from the stimuli.

Another mechanism that possibly supports blindsight is the interhemispheric integration and cooperation. Two experiments in blindsight patients showed that, when responding to stimuli in either visual field with one of the hands, for the intact visual field the RT was shorter when the hand was controlled by the motor cortex ipsilateral to the hemisphere processing the visual information; for the blind visual field the opposite pattern of RT emerged, indicating that the visual information needed additional processing time in the intact hemisphere, which involved the parietal and premotor areas through the corpus callosum (Celeghin et al., 2015; Celeghin et al., 2017). In our current experiment, participants used different fingers of the same hand to respond, thus we were not able to make direct comparisons. Instead we could examine whether a Simon effect was present, which may also be related to interhemispheric cooperation. With a Simon effect, ipsilateral RT to the target side is facilitated. In our data we did not find a significant Simon effect of the responding side and visual field, nor any interaction to other factors though. For the CFS 


\section{Chapter 3}

mechanism which mainly involves interocular competition within the same hemispheres, it is not yet clear whether interhemispheric cooperation would also be involved. Further CFS studies combining both the RTE and two-hand responses would be suitable to investigate this hypothesis.

With the CFS paradigm, two recent studies used a similar bilateral presentation design. One presented one arrow and 4 flanker arrows dichoptically, with the flanker arrows either masked with CFS or not masked. They found that the non-masked flanker arrows with direction incongruent to the target arrow slowed down the RT for the target, but this effect was abolished when the flanker arrows were suppressed, that no difference was found comparing to the conditions without flankers (Wu et al., 2015). Another study faded in fearful and happy faces under CF-suppression $600 \mathrm{~ms}$ before presenting the target (thus was a much longer prime), while participants categorized the emotion of a visible target face briefly presented for $200 \mathrm{~ms}$. The study found a facilitation effect on RT for the unconscious faces with congruent emotions ( $\mathrm{Ye}, \mathrm{He}, \mathrm{Hu}, \mathrm{Yu}, \&$ Wang, 2014). The difference between the results of these two studies may therefore be related to the different levels of their suppression under CFS, that one of them showed very strong suppression, and the other used priming effects beside the bilateral presentation.

In our case, since the traditional redundant target gain effect shown with backward-masking was not seen here under CFS, residual vision under CFS suppression is likely to be abolished more thoroughly than that with backward-masking. However, as the unconscious fearful bodies facilitate the processing of conscious angry bodies, does the amount of information transmitted under CFS allow integration between the two emotions? Because the angry and fearful bodies together have not been tested behaviorally under either conscious or masking conditions, future behavioral experiments with healthy participants would shed light on the level of integrations between these two emotions, and behavioral CFS studies with bilateral presentation are needed to see whether unconscious fearful bodies would also facilitate non-emotion-related tasks in a manner similar to fearful faces for hemianopia patients.

When brain activity of healthy participants were observed under fMRI, consciously seeing two incongruent body expressions (fear and happy) induced weaker activity across the brain than seeing two congruent body expressions, indicating interference between the incongruent emotions (de Borst \& de Gelder, 2016). When participants consciously observed two actors interacting in a violent social scene, participants' attention on the aggressor induced activation of body-processing areas (extrastriate body area) and emotion-related areas (Van den Stock, Hortensius, Sinke, Goebel, \& de Gelder, 2015). In our study, although two body stimuli were bilaterally presented, with one of them unseen, the cognitive process induced by our design may be different from these two conscious viewing situations. First, we did not observe a facilitation effect by the congruent emotions, or interference by the incongruent emotions. Second, there was no intrinsic social interaction between our bilaterally presented stimuli: both were facing the observer (participant), but not facing each other. Although in our case the participant's percept could be treated as "attending to an aggressor (angry) or a victim (fearful)", both cases were "interacting" with the observer, this percept was the same when there was only one unilateral stimulus presented, for which we did not find a main effect of the consciously perceived emotion. Thus our results suggest 
that unconscious perception of bodies under CFS is very different from perception that is fully conscious, and its brain substrates still await future examinations.

\section{General discussion}

In three behavioral experiments with the CFS paradigm, we expected to find dissociable effects of unconscious bodies expressing fearful, happy, angry or neutral expressions. We did not find a priming effect of unconscious fearful and happy bodies/faces; neither did we find dissociation between detection performances and confidence ratings for unconscious angry or neutral bodies, possibly due to a floor effect. These negative results indicate that the CFS paradigm has very strong suppression. Our findings in the bilateral presentation of fearful and angry bodies experiment are consistent with this, where the unconscious bodies were processed to an extent, but were not to the level that would allow a classical redundant target effect to appear.

The information of stimuli that transpire under CFS is still under debate (for reviews see Gayet, Van der Stigchel, and Paffen (2014); (Sterzer, Stein, Ludwig, Rothkirch, \& Hesselmann, 2014). Functional MRI studies showed that faces under CFS were processed to a less extent in the fusiform areas (Fang \& He, 2005), but fearful faces could show activation in the amygdala (Jiang \& He, 2006). On the other hand, the activation was preserved in dorsal areas for tools, but not for faces (Fang \& He, 2005, but see (Hesselmann \& Malach, 2011; Ludwig, Kathmann, Sterzer, \& Hesselmann, 2015, and the discussion in Chapter 5). The activation in different areas had been used as evidence that the emotional or categorical information of the stimuli transpired. However, behavioral CFS experiments indicate that the advantage of fearful faces breaking from suppression was induced mainly by lower level features, especially the contrast in the eye regions (Gray et al., 2013; Yang et al., 2007), although it cannot be denied that the low-level features are directly contributing to our perception of emotions.

A range of different methods have been used in the consciousness literature to render stimulus unconscious, and several studies had made efforts to compare them. Almeida et al. (2013) observed an influence of happy faces on the likability rating for Chinese characters under backward masking, but did not observe this effect under CFS. Faivre et al. (2012) also compared CFS with conscious viewing and several other non-conscious paradigms, including gaze-contingent crowding and masking, and showed that the preference bias for angry, neutral, happy emotional faces were different across these methods. Another recent fMRI study further compared the brain responses between CFS and chromatic flicker fusion, and found the categorical information of stimuli could be decoded from temporal and frontal areas under chromatic flicker fusion, but not with CFS (Fogelson, Kohler, Miller, Granger, \& Tse, 2014). We observed in our current study of bilateral presentation, that the facilitation of incongruent emotions was also different from the traditional redundant target effect under backward masking or visible conditions. The evidence all points to a difference in the mechanisms rendering stimulus unconscious, thus the phenomenon of being visually unconscious of a stimulus, is likely to involve multiple stages and processes. 


\section{Chapter 3}

For the same reason, the blindsight, hemianopia without blindsight, and neglect phenomena are also likely to involve different lesion-induced mechanisms or even neural reorganizations, and the links of them to CFS and other methods rendering stimuli unconscious are yet to be fully established. We observed similarity of CFS to hemianopia patients without blindsight, but not to blindsight patients or neglect patients. Similar to hemianopia patients not displaying above-chance performance for a range of visual stimuli, thus regarded truly "blind" but nevertheless showed a facilitation of unconscious fearful faces, it is also possible for the methods applied to healthy participants, that the stimulus information was processed in some way in the brain, but did not induce dissociation or was not captured in either behavioral measures or BOLD signal measures. For example, the decodability of categories is only one dimension of the stimulus properties, while emotional activation is another dimension, but amygdala activation per se does not serve as a good indicator of emotional information being present, given its involvement in attention, value representation and decision making (Pessoa, 2010). Apart from finding a sensitive behavioral measure with healthy participants and patients, further $\mathrm{FMRI}$ and EEG/MEG research with more sensitive measures or neural signatures for unconscious processing would be necessary for better understanding unconscious processes.

\section{References}

Almeida, J., Pajtas, P. E., Mahon, B. Z., Nakayama, K., \& Caramazza, A. (2013). Affect of the unconscious: visually suppressed angry faces modulate our decisions. Cogn Affect Behav Neurosci, 13(1), 94-101. doi: 10.3758/s13415-012-0133-7

Bertini, C., Cecere, R., \& Ladavas, E. (2013). I am blind, but I "see" fear. Cortex, 49(4), 985-993. doi: 10.1016/j.cortex.2012.02.006

Bertini, C., Cecere, R., \& Ladavas, E. (2017). Unseen fearful faces facilitate visual discrimination in the intact field. Neuropsychologia. doi: 10.1016/j.neuropsychologia.2017.07.029

Brainard, D. H. (1997). The psychophysics toolbox. Spatial vision, 10, 433-436.

Burra, N., Hervais-Adelman, A., Celeghin, A., de Gelder, B., \& Pegna, A. J. (2017). Affective blindsight relies on low spatial frequencies. Neuropsychologia. doi: 10.1016/j.neuropsychologia.2017.10.009

Cecere, R., Bertini, C., \& Ladavas, E. (2013). Differential contribution of cortical and subcortical visual pathways to the implicit processing of emotional faces: a tDCS study. J Neurosci, 33(15), 6469-6475. doi: 10.1523/jneurosci.3431-12.2013

Cecere, R., Bertini, C., Maier, M. E., \& Ladavas, E. (2014). Unseen fearful faces influence face encoding: evidence from ERPs in hemianopic patients. J Cogn Neurosci, 26(11), 2564-2577. doi: 10.1162/jocn_a_00671

Celeghin, A., Barabas, M., Mancini, F., Bendini, M., Pedrotti, E., Prior, M., ... Marzi, C. A. (2015). Speeded manual responses to unseen visual stimuli in hemianopic patients: what kind of blindsight? Conscious Cogn, 32, 6-14. doi: 10.1016/j.concog.2014.07.010

Celeghin, A., Diano, M., de Gelder, B., Weiskrantz, L., Marzi, C. A., \& Tamietto, M. (2017). Intact hemisphere and corpus callosum compensate for visuomotor functions after early visual 


\section{Three behavioral CFS experiments}

cortex damage. Proc Natl Acad Sci U S A, 114(48), E10475-E10483. doi:

10.1073/pnas.1714801114

Corbetta, M., \& Shulman, G. L. (2011). Spatial neglect and attention networks. Annu Rev Neurosci, 34, 569-599. doi: 10.1146/annurev-neuro-061010-113731

Dael, N., Mortillaro, M., \& Scherer, K. R. (2012). Emotion expression in body action and posture. Emotion, 12(5), 1085-1101. doi: 10.1037/a0025737

Danckert, J., \& Rossetti, Y. (2005). Blindsight in action: what can the different sub-types of blindsight tell us about the control of visually guided actions? Neurosci Biobehav Rev, 29(7), 1035-1046. doi: 10.1016/j.neubiorev.2005.02.001

de Borst, A. W., \& de Gelder, B. (2016). Clear signals or mixed messages: inter-individual emotion congruency modulates brain activity underlying affective body perception. Soc Cogn Affect Neurosci, 11(8), 1299-1309. doi: 10.1093/scan/nsw039

de Gelder, B., Morris, J. S., \& Dolan, R. J. (2005). Unconscious fear influences emotional awareness of faces and voices. Proc Natl Acad Sci U S A, 102(51), 18682-18687. doi: 10.1073/pnas.0509179102

de Gelder, B., Pourtois, G., van Raamsdonk, M., Vroomen, J., \& Weiskrantz, L. (2001). Unseen stimuli modulate conscious visual experience: evidence from inter-hemispheric summation. Neuroreport, 12(2), 385-391.

de Gelder, B., Van den Stock, J., Meeren, H. K., Sinke, C. B., Kret, M. E., \& Tamietto, M. (2010). Standing up for the body. Recent progress in uncovering the networks involved in the perception of bodies and bodily expressions. Neurosci Biobehav Rev, 34(4), 513-527. doi: 10.1016/j.neubiorev.2009.10.008

de Gelder, B., Vroomen, J., Pourtois, G., \& Weiskrantz, L. (1999). Non-conscious recognition of affect in the absence of striate cortex. Neuroreport, 10(18), 3759-3763.

Driver, J., \& Vuilleumier, P. (2001). Perceptual awareness and its loss in unilateral neglect and extinction. Cognition, 79(1-2), 39-88.

Faivre, N., Berthet, V., \& Kouider, S. (2012). Nonconscious influences from emotional faces: a comparison of visual crowding, masking, and continuous flash suppression. Front Psychol, 3 , 129. doi: 10.3389/fpsyg.2012.00129

Fang, F., \& He, S. (2005). Cortical responses to invisible objects in the human dorsal and ventral pathways. Nat Neurosci, 8(10), 1380-1385. doi: 10.1038/nn1537

Fogelson, S. V., Kohler, P. J., Miller, K. J., Granger, R., \& Tse, P. U. (2014). Unconscious neural processing differs with method used to render stimuli invisible. Front Psychol, 5, 601. doi: 10.3389/fpsyg.2014.00601

Gayet, S., Van der Stigchel, S., \& Paffen, C. L. E. (2014). Breaking continuous flash suppression: competing for consciousness on the pre-semantic battlefield. Front Psychol, 5, 460. doi: 10.3389/fpsyg.2014.00460

Georgy, L., Celeghin, A., Marzi, C. A., Tamietto, M., \& Ptito, A. (2016). The superior colliculus is sensitive to gestalt-like stimulus configuration in hemispherectomy patients. Cortex, 81,151 161. doi: 10.1016/j.cortex.2016.04.018

Gray, K. L., Adams, W. J., Hedger, N., Newton, K. E., \& Garner, M. (2013). Faces and awareness: lowlevel, not emotional factors determine perceptual dominance. Emotion, 13(3), 537-544. doi: $10.1037 / \mathrm{a} 0031403$

Hesselmann, G., \& Malach, R. (2011). The link between fMRI-BOLD activation and perceptual awareness is "stream-invariant" in the human visual system. Cereb Cortex, 21(12), 2829-2837. doi: $10.1093 /$ cercor/bhr085

Houghton, G., \& Grange, J. A. (2011). CDF-XL: computing cumulative distribution functions of reaction time data in Excel. Behav Res Methods, 43(4), 1023-1032. doi: 10.3758/s13428-011-0119-3 


\section{Chapter 3}

Jiang, Y., \& He, S. (2006). Cortical responses to invisible faces: dissociating subsystems for facialinformation processing. Curr Biol, 16(20), 2023-2029. doi: 10.1016/j.cub.2006.08.084

Kouider, S., \& Dehaene, S. (2007). Levels of processing during non-conscious perception: a critical review of visual masking. Philos Trans R Soc Lond B Biol Sci, 362(1481), 857-875. doi: $10.1098 /$ rstb.2007.2093

Langner, O., Dotsch, R., Bijlstra, G., Wigboldus, D. H., Hawk, S. T., \& van Knippenberg, A. (2010). Presentation and validation of the Radboud Faces Database. Cognition and Emotion, 24(8), 1377-1388.

Lapate, R. C., Rokers, B., Tromp, D. P. M., Orfali, N. S., Oler, J. A., Doran, S. T., . . Davidson, R. J. (2016). Awareness of Emotional Stimuli Determines the Behavioral Consequences of Amygdala Activation and Amygdala-Prefrontal Connectivity. Sci Rep, 6, 25826. doi: 10.1038/srep25826

Lau, H. C., \& Passingham, R. E. (2006). Relative blindsight in normal observers and the neural correlate of visual consciousness. Proc Natl Acad Sci U S A, 103(49), 18763-18768. doi: $10.1073 /$ pnas.0607716103

Lohse, M., \& Overgaard, M. (2017). Emotional priming depends on the degree of conscious experience. Neuropsychologia. doi: 10.1016/j.neuropsychologia.2017.10.028

Ludwig, K., Kathmann, N., Sterzer, P., \& Hesselmann, G. (2015). Investigating category- and shapeselective neural processing in ventral and dorsal visual stream under interocular suppression. Hum Brain Mapp, 36(1), 137-149. doi: 10.1002/hbm.22618

Mazzi, C., Bagattini, C., \& Savazzi, S. (2016). Blind-Sight vs. Degraded-Sight: Different Measures Tell a Different Story. Front Psychol, 7, 901. doi: 10.3389/fpsyg.2016.00901

Mazzi, C., Tagliabue, C. F., Mazzeo, G., \& Savazzi, S. (2018). Reliability in reporting perceptual experience: Behaviour and electrophysiology in hemianopic patients. Neuropsychologia. doi: 10.1016/j.neuropsychologia.2018.01.017

Mendez-Bertolo, C., Moratti, S., Toledano, R., Lopez-Sosa, F., Martinez-Alvarez, R., Mah, Y. H., . . . Strange, B. A. (2016). A fast pathway for fear in human amygdala. Nat Neurosci, 19(8), $1041-$ 1049. doi: $10.1038 / \mathrm{nn} .4324$

Miller, J. (1982). Divided attention: evidence for coactivation with redundant signals. Cogn Psychol, 14(2), 247-279.

Pelli, D. G. (1997). The VideoToolbox software for visual psychophysics: Transforming numbers into movies. Spatial vision, 10(4), 437-442.

Pessoa, L. (2010). Emotion and cognition and the amygdala: from "what is it?" to "what's to be done?". Neuropsychologia, 48(12), 3416-3429. doi: 10.1016/j.neuropsychologia.2010.06.038

Raab, D. H. (1962). Statistical facilitation of simple reaction times. Trans N Y Acad Sci, 24, 574-590.

Ramsøy, T. Z., \& Overgaard, M. (2004). Introspection and subliminal perception. Phenomenology and the Cognitive Sciences, 3(1), 1-23. doi: 10.1023/B:PHEN.0000041900.30172.e8

Ratcliff, R. (1979). Group reaction time distributions and an analysis of distribution statistics. Psychol Bull, 86(3), 446-461.

Snodgrass, J. G., \& Corwin, J. (1988). Pragmatics of measuring recognition memory: applications to dementia and amnesia. Journal of Experimental Psychology: General, 117(1), 34.

Sterzer, P., Stein, T., Ludwig, K., Rothkirch, M., \& Hesselmann, G. (2014). Neural processing of visual information under interocular suppression: a critical review. Front Psychol, 5, 453. doi: 10.3389/fpsyg.2014.00453

Stienen, B. M., \& de Gelder, B. (2011). Fear detection and visual awareness in perceiving bodily expressions. Emotion, 11(5), 1182-1189. doi: 10.1037/a0024032

Tamietto, M., Castelli, L., Vighetti, S., Perozzo, P., Geminiani, G., Weiskrantz, L., \& de Gelder, B. (2009). Unseen facial and bodily expressions trigger fast emotional reactions. Proc Natl Acad Sci U S A, 106(42), 17661-17666. doi: 10.1073/pnas.0908994106 
Tamietto, M., Cauda, F., Celeghin, A., Diano, M., Costa, T., Cossa, F. M., . . de de Gelder, B. (2015). Once you feel it, you see it: Insula and sensory-motor contribution to visual awareness for fearful bodies in parietal neglect. Cortex, 62, 56-72. doi: 10.1016/j.cortex.2014.10.009

Tamietto, M., \& de Gelder, B. (2008). Affective blindsight in the intact brain: neural interhemispheric summation for unseen fearful expressions. Neuropsychologia, 46(3), 820-828. doi: 10.1016/j.neuropsychologia.2007.11.002

Tamietto, M., Geminiani, G., Genero, R., \& de Gelder, B. (2007). Seeing fearful body language overcomes attentional deficits in patients with neglect. J Cogn Neurosci, 19(3), 445-454.

Tamietto, M., Latini Corazzini, L., de Gelder, B., \& Geminiani, G. (2006). Functional asymmetry and interhemispheric cooperation in the perception of emotions from facial expressions. Exp Brain Res, 171(3), 389-404. doi: 10.1007/s00221-005-0279-4

Tong, F., Meng, M., \& Blake, R. (2006). Neural bases of binocular rivalry. Trends Cogn Sci, 10(11), $502-$ 511. doi: 10.1016/j.tics.2006.09.003

Tsuchiya, N., \& Koch, C. (2005). Continuous flash suppression reduces negative afterimages. Nat Neurosci, 8(8), 1096-1101. doi: 10.1038/nn1500

Tsuchiya, N., Koch, C., Gilroy, L. A., \& Blake, R. (2006). Depth of interocular suppression associated with continuous flash suppression, flash suppression, and binocular rivalry. J Vis, 6(10), 10681078. doi: $10.1167 / 6.10 .6$

Van den Stock, J., Hortensius, R., Sinke, C., Goebel, R., \& de Gelder, B. (2015). Personality traits predict brain activation and connectivity when witnessing a violent conflict. Sci Rep, 5, 13779. doi: 10.1038/srep13779

Van den Stock, J., Tamietto, M., Sorger, B., Pichon, S., Grézes, J., \& de Gelder, B. (2011). Corticosubcortical visual, somatosensory, and motor activations for perceiving dynamic whole-body emotional expressions with and without striate cortex (V1). Proceedings of the National Academy of Sciences, 108(39), 16188-16193.

Van den Stock, J., Tamietto, M., Zhan, M., Heinecke, A., Hervais-Adelman, A., Legrand, L. B., ... de Gelder, B. (2014). Neural correlates of body and face perception following bilateral destruction of the primary visual cortices. Front Behav Neurosci, 8, 30. doi: 10.3389/fnbeh.2014.00030

Vuilleumier, P., Armony, J. L., Clarke, K., Husain, M., Driver, J., \& Dolan, R. J. (2002). Neural response to emotional faces with and without awareness: event-related fMRI in a parietal patient with visual extinction and spatial neglect. Neuropsychologia, 40(12), 2156-2166.

Vuilleumier, P., \& Schwartz, S. (2001). Emotional facial expressions capture attention. Neurology, 56(2), 153-158.

Weiskrantz, L. (1986). Blindsight: A case study and implications.

Whalen, P. J., Kagan, J., Cook, R. G., Davis, F. C., Kim, H., Polis, S., .. Johnstone, T. (2004). Human amygdala responsivity to masked fearful eye whites. Science, 306(5704), 2061. doi: 10.1126/science.1103617

Williams, M. A., \& Mattingley, J. B. (2004). Unconscious perception of non-threatening facial emotion in parietal extinction. Exp Brain Res, 154(4), 403-406. doi: 10.1007/s00221-003-1740-x

Wu, Q., Lo Voi, J. T. H., Lee, T. Y., Mackie, M.-A., Wu, Y., \& Fan, J. (2015). Interocular suppression prevents interference in a flanker task. Front Psychol, 6, 1110. doi: 10.3389/fpsyg.2015.01110

Yang, E., Zald, D. H., \& Blake, R. (2007). Fearful expressions gain preferential access to awareness during continuous flash suppression. Emotion, 7(4), 882-886. doi: 10.1037/15283542.7.4.882

Ye, X., He, S., Hu, Y., Yu, Y. Q., \& Wang, K. (2014). Interference between conscious and unconscious facial expression information. PLoS One, 9(8), e105156. doi: 10.1371/journal.pone.0105156 


\section{Chapter 3}

Zhan, M., Hortensius, R., \& de Gelder, B. (2015). The Body as a Tool for Anger Awareness-Differential Effects of Angry Facial and Bodily Expressions on Suppression from Awareness. PLoS One, 10(10), e0139768. 


\section{Chapter 4}

\section{Influence of continuous flash suppression mask frequency on stimulus visibility}

Corresponding manuscript:

Zhan, M., Engelen, T., \& de Gelder, B. (under revision in Neuropsychologia). Influence of continuous flash suppression mask frequency on stimulus visibility 


\section{Chapter 4}

\section{Abstract}

The continuous flash suppression (CFS) paradigm is increasingly used in consciousness research, but its mechanisms are still not fully understood. To better understand its temporal properties, we presented the CFS masks at 9 frequencies, and examined their influence on stimuli visibility, while taking into account the inter-individual variability and the change of CFS suppression as the experiment progressed. The frequencies consisted of fundamental frequencies of 3,4 and $5 \mathrm{~Hz}$, and their $2^{\text {nd }}$ and $3^{\text {rd }}$ harmonics, which included the $10 \mathrm{~Hz}$ frequency typically used in most of the CFS studies. We found that the suppression of stimulus awareness was stronger under 4,6 and $8 \mathrm{~Hz}$ than $10 \mathrm{~Hz}$. After controlling for inter-individual variability with mixed-effects analysis, we found that the number of seen trials was lower for the $4 \mathrm{~Hz}$-basis frequencies than the $5 \mathrm{~Hz}$ ones, and was lower for the $2^{\text {nd }}$ than $3^{\text {rd }}$ harmonic. We propose that this may be caused by an interaction between the CFS masks and the ongoing sampling of the attentional mechanism. Examining individual data, we also found a time effect that the participants saw significantly more stimuli as the experiment progressed. Our results suggest that these factors need to be taken care of in future CFS studies in order to achieve optimal visual awareness suppression and ensure the generalizability of results. 


\section{Introduction}

The study of perception outside awareness has advanced our understandings of brain functions. Studies of brain lesioned-patients uncovered phenomena such as blindsight (Celeghin, de Gelder, \& Tamietto, 2015; de Gelder, Vroomen, Pourtois, \& Weiskrantz, 1999; Van den Stock, Tamietto, Hervais-Adelman, Pegna, \& de Gelder, 2013; Weiskrantz, 1986), visual agnosia (Farah, 2004), optic ataxia (Jakobson, Archibald, Carey, \& Goodale, 1991), leading to the establishment of the two-stream visual processing model (Milner \& Goodale, 2006). The blindsight phenomenon was of particular interest, showing that patients with V1 lesion could still report and react above chance to visual stimuli, without being conscious of the visual stimuli being present. Given the theoretical importance of vision without consciousness and because blindsight patients are rare, efforts have been made to establish and study similar phenomena in neurologically intact participants.

Continuous flash suppression (CFS) has been a prime candidate paradigm for such purposes (Tsuchiya \& Koch, 2005). CFS utilizes dichoptic presentation of stimuli, and can successfully suppress visual awareness of a static lower-contrast target stimulus in one eye for up to several seconds, by presenting a dynamic and high-contrast flashing mask in the other eye (Tsuchiya, Koch, Gilroy, \& Blake, 2006). Despite the potent suppression effect, some information of the stimuli could still transpire to higher visual areas. Because of this blindsight-like property, CFS has been increasingly used as a tool for consciousness research, to study both the non-conscious processing of simple stimuli like checkerboards and Gabor patches, as well as more complex stimuli like faces and words, which could also contain emotional or semantic contents in addition to the visual form (e.g. (Costello, Jiang, Baartman, McGlennen, \& He, 2009; Jiang \& He, 2006; Yang, Zald, \& Blake, 2007).

Despite increasingly wide applications of the CFS paradigm in consciousness research, there are concerns regarding the generalizability of the results obtained from this paradigm. One concern relates to the fact that its suppression mechanisms are still not fully understood. Investigations on the spatial domain of both the stimuli and the mask pattern have demonstrated that low-level properties such as contrast can influence whether a stimulus is perceived by the participant (Gray, Adams, Hedger, Newton, \& Garner, 2013; Yang et al., 2007). At the same time, the strength of suppression is related to the spatial frequencies of the dynamic mask pattern as well as that of the stimulus (Stein, Seymour, Hebart, \& Sterzer, 2014; Yang \& Blake, 2012). However, so far investigations on the temporal dynamics are still scarce, especially concerning the temporal frequencies of the dynamic mask pattern.

In the influential article that established the CFS paradigm (Tsuchiya \& Koch, 2005), the authors used a $10 \mathrm{~Hz}$-flash frequency of the dynamic mask pattern. Their choice was based on the observation in a separate test with 4 participants naive to the paradigm, that the optimal suppression length was obtained with a flash frequency of $\sim 3-12 \mathrm{~Hz}$ (the 10 tested frequencies ranged from 0.78 to $100 \mathrm{~Hz}$ ). So far most published CFS studies have used this flash frequency, following their example.

Four recent studies investigated the influence of flash frequency on the visual awareness of stimuli, spanning different frequency ranges and measuring different dependent variables 


\section{Chapter 4}

(Drewes, Zhu, \& Melcher, 2018; Han, Lunghi, \& Alais, 2016; Kaunitz, Fracasso, Skujevskis, \& Melcher, 2014; Zhu, Drewes, \& Melcher, 2016). Kaunitz et al. tested 5 frequency levels (5.3, $8.5,10.6,16.6$ and $28.5 \mathrm{~Hz}$ ) on the visibility of transiently presented checkerboard targets, and found a general decrease of seen trials as the frequency increased (Kaunitz et al., 2014). Zhu et al. used more complex stimuli of faces and houses, in addition to simple symbols, and measured the break-through contrasts of the stimuli at 10 frequency levels of the mask $(0,1$, $3,5,7,10,13,16,20,32 \mathrm{~Hz})$. They found that the stimuli contrast showed a skewed normal curve across frequencies, peaking around $6 \mathrm{~Hz}$ for all four kinds of stimuli tested (higher suppression effect there) (Zhu et al., 2016). In a follow-up study, they further found that the optimal temporal mask frequency increased while the spatial density of the mask decreased (Drewes et al., 2018). Han et al. did not examine individual flash frequencies directly; instead they used a temporal filter on the noise masks $(0.375,0.75,1.5,3,6.25,12.5,25 \mathrm{~Hz})$ and examined the suppression duration for 4 images of natural stimuli. They found the suppression duration peaked at very low frequencies around $1 \mathrm{~Hz}$, although the contrast sensitivity curve across frequencies did not show the same pattern, which peaked at $6.25 \mathrm{~Hz}$ (Han et al., 2016). These studies did not optimally sample the frequency range found by Tsuchiya \& Koch (2005), and did not reach conclusions about a consistent frequency range needed for strong suppression.

Another concern about the generalizability of results obtained with the CFS paradigm relates to the substantial inter-individual variability in suppression time observed by recent studies, not only for simple stimuli (Yamashiro et al., 2014), but also for complex stimuli varying on social dimensions (Getov, Kanai, Bahrami, \& Rees, 2015).

To better understand the properties of the CFS paradigm, in the current study, we examined the effect of flash frequencies on the visibility of stimuli, while taking into account the possible inter-individual variability. We chose 9 frequency levels from 3-15 $\mathrm{Hz}$ on two objectives. This allowed us to better sample the optimal frequency range around 3-12 Hz found by Tsuchiya \& Koch (2005), also including the routinely used $10 \mathrm{~Hz}$ frequency. It also allowed us to test hypotheses on the relationships between stimuli visibility and the frequencies. We hypothesized 3 possible relationships: 1) monotonic, meaning that the stimulus visibility may increase (or decrease) while the flash frequency increases; 2 ) quadratic, meaning that the stimulus visibility may peak in the mid-range frequencies, while being low at both very low and very high frequencies (or the other way around); 3 ) as recent research in visual attentional mechanisms showed, stimuli are being sampled the frequencies of around $4 \mathrm{~Hz}$ and $8 \mathrm{~Hz}$ (Buschman \& Kastner, 2015; VanRullen, 2016), which does not follow the previous two hypotheses, but happen to be a fundamental frequency and its $2^{\text {nd }}$ harmonic. Thus in our case there is the possibility that stimulus visibility may similarly be related to the fundamental frequencies of the flash masks and their harmonics. To be able to test all these 3 hypotheses, we chose fundamental frequencies of 3,4 , and 5 $\mathrm{Hz}$, and their $2^{\text {nd }}$ and $3^{\text {rd }}$ harmonics $(6,8,10 \mathrm{~Hz}$ and $9.23,12,15 \mathrm{~Hz}$ respectively). The $9.23 \mathrm{~Hz}$ (approximation of $9 \mathrm{~Hz}$ ) was due to LCD monitor refresh-rate limits. For the target stimuli, we used 10 whole body images displaying neutral actions. Similar to faces, human bodies are salient and behaviourally-relevant stimuli. They could be processed outside visual awareness in both blindsight patients with V1 lesions (Van den Stock et al., 2014), and in normal participants under CFS, showing longer suppression time than faces (Stein, Sterzer, \& Peelen, 
2012; Zhan, Hortensius, \& de Gelder, 2015). Using neutral bodies allowed us to maximize stimuli relevance, while avoiding ceiling/floor effects that too many (faces) or too few stimuli (low-level visual stimuli without much behavioural relevance, such as Gabor patches) are seen. We performed mixed-effects analysis to examine and control inter-individual variability, and we performed correlation analysis on individual data to examine the confounding time effect of experiment progression on stimulus visibility.

\section{Materials and methods}

\section{Participants}

Fifty-five female participants (age range 17-28) were recruited from the campus of Maastricht University and took part in the study. Most of them were naïve to the CFS paradigm. We tested female participants only, because this whole session of the current study served as a screening test for another experiment not reported here, for which previous research has reported gender differences. Participants all had normal or correctedto-normal visual acuity, normal stereo color vision, and no history of neurological disorders. They gave written consent before participation, and received either monetary rewards or course credits after participation. The experiment was approved by the ethical committee of Maastricht University, and was carried out in accordance to the Declaration of Helsinki.

\section{Stimuli}

Gray-scale images of neutral faces and bodies were used. For the face stimuli, 10 identities ( 5 females) were chosen from the Radboud Face Database (Langner et al., 2010), aligned at the eye level. For the body stimuli, 10 identities (males only) displaying an action of talking on the phone were chosen from the set developed by Stienen and de Gelder (2011), aligned at the feet level, with facial information removed. The face and body stimuli spanned visual angles of $2.83^{\circ} \times 2.16^{\circ}$ and $4.43^{\circ} \times 1.88^{\circ}$ respectively, and were embedded in a gray rectangle background $\left(240 \times 160\right.$ pixels, visual angle $6.73^{\circ} \times 4.48^{\circ}$, RGB value $\left.128,128,128\right)$. These stimuli were a subset of the stimuli used in a previous CFS study, where we found that the suppression time for the face stimuli were shorter than the body stimuli (Zhan et al., 2015). In the current study, the face stimuli were used for determining the eye dominance for each participant, to facilitate break from suppression and to have an adequate number of seen trials in a relatively short test. We then used the body stimuli in the main experiment of flash frequencies, to diminish possible ceiling effects of "seen".

Six-hundred unique colored mask patterns were constructed, by randomly drawing small rectangles of different colors (the heights and widths were within $2^{\circ}$ visual angles) in the area of $240 \times 160$ pixels. In each trial of the experiments, the dynamic mask patterns were randomly drawn from this pool without replacement. 


\section{Chapter 4}

\section{Dichoptic presentation}

The stimuli and the dynamic mask patterns were presented in Matlab R2013b (the MathWorks, Natick, MA, USA) with Psychtoolbox (Brainard, 1997; Pelli, 1997), on an LCD screen (Acer VG248, resolution $=1920 \times 1080$, refresh rate $=120 \mathrm{~Hz}$ ). To aid the free-fuse of the dichoptic views for the participants, two black rectangle frames $(240 \times 160$ pixels, 10 pixels thick) were projected side by side in the center of the screen, 254 pixels apart from each other. A white fixation cross was presented at the center of each rectangle frame. In each trial, the dynamic mask pattern and the stimulus were projected separately into one rectangle frame. Participants viewed the stimuli on the screen through a pair of prism glasses (diopter=7) (Schurger, 2009) while resting their chin in a chinrest, with a viewing distance of about $57 \mathrm{~cm}$. A cardboard was placed between the screen and the participant, dividing the screen into two equal halves, so that each eye of the participant saw one half of the screen without crosstalk. Participants were asked to free-fuse the two views in one stable rectangle box, without drifting apart. For participants who could not free-fuse the views with the glasses of diopter $=7$, glasses of diopter $=5$ were used instead.

\section{Procedure}

The experimental session consisted of an eye-dominance test of $6 \mathrm{~min}$, and the main experiment lasting 49 to 58 min (depending on response times of the participant, and the self-paced resting periods between experimental blocks). Both tests started after a stable free-fusing of two views was established. Participants were instructed to keep fixation on the fixation cross throughout the whole experimental session, keeping their head as still as possible, and not to blink during stimulus presentation if possible. They reported their subjective awareness of the stimuli in both tests by pressing the 1 (seen) and 2 (unseen) keys on the keyboard always with the left hand. They were instructed beforehand that they should respond "seen" as long as they saw some part of the stimulus during the presentation of the mask patterns. For trials in both tests, the response window was $2 \mathrm{~s}$, and the inter-trial-interval was $1 \mathrm{~s}$.

In the eye dominance test, neutral faces of 10 identities (half female) were presented to the participants under CFS, with the dynamic mask pattern flashing at $10 \mathrm{~Hz}$. Each stimulus image was presented to each eye 3 times, resulting in a total of 60 trials. The order of the stimuli presentation and the eye the stimuli were projected to were both randomized. For each trial, the face stimulus was faded in from $0 \%$ contrast to full contrast in $1.5 \mathrm{~s}$, maintained at full contrast for $1 \mathrm{~s}$, and then faded out to $0 \%$ contrast in $0.5 \mathrm{~s}$. The full contrasts of the faces were to facilitate the breaking from suppression during stimuli presentation, in order to have an adequate number of seen trials for each eye. The numbers of seen trials per eye were counted, and the eye with the higher number of seen trials was assigned as the dominant eye for that participant. When the numbers of seen trials were equal between both eyes, the right eye was assigned as the dominant eye (this was the case for 6 participants, 3 of whom were included in further analysis).

In the main experiment, neutral body stimuli of 10 males were presented to the participants' non-dominant eye under CFS (19 into the left eye and 18 into the right eye), while the dynamic mask varied in 9 different frequencies: 3, 4, 5, 6, 8, 9.23, 10, 12, $15 \mathrm{~Hz}$ respectively. The $9.23 \mathrm{~Hz}$ condition was limited by the LCD screen's refresh rate, during which each 
dynamic mask pattern stayed on the screen for 13 frames. Each stimulus was presented 4 times per flash frequency, resulting in a total of 360 trials. The order for both the body stimuli and the frequencies was randomized across the whole experiment, and the trials were then split in to 6 blocks after randomization. To avoid a ceiling effect of "seen" responses, for each trial, the body stimulus was faded in from $0 \%$ to $50 \%$ contrast in $1.5 \mathrm{~s}$, stayed at $50 \%$ contrast for $1 \mathrm{~s}$, and faded out to $0 \%$ in $1.5 \mathrm{~s}$. The dynamic mask was presented for another $1 \mathrm{~s}$ after stimuli offset, to avoid perception of stimulus afterimages. In total the dynamic mask was kept on screen for $5 \mathrm{~s}$ per trial.

\section{Data analyses}

The data of 37 participants (mean age $=20.16, S D=1.91$, range 17-28) were included in the subsequent analyses. For the participants whose data were excluded from analysis, 2 participants did not complete the main experiment due to not being able to maintain the merging of the two boxes. The data of the other 16 participants were excluded for ceiling or floor effects based on these criteria: 2 participants missed responses for more than $10 \%$ of all trials ( 36 trials), 12 participants responded "seen" for more than $90 \%$ of the trials (324 trials), and 2 participants responded "unseen" for more than $90 \%$ of the trials (324 trials).

For the data of the main experiment, within each participant the numbers of seen trials per flash frequency were counted, and the average number of seen trials was computed across 9 frequencies. This average number was then subtracted from the number of seen trials for each frequency (which we refer to as "centering" in the subsequent text). After centering, the numbers of seen trials became normally distributed across participants, and the varying effect of CF-suppression strength across participants was removed (the baseline became 0 , and we examined whether for certain frequencies the participants see more/less trials than they see on average, e.g. value " 1 " for one participant at one frequency would mean that the participant consciously saw one more trial than she saw on average across frequencies). We performed two repeated-measures ANOVAs in SPSS with the centered number of seen trials. The first ANOVA had one factor "flash frequency" with 9 levels; the second ANOVA had the factor "fundamental frequency" $(3,4,5 \mathrm{~Hz})$, and the factor "harmonic levels" (fundamental frequency, their $2^{\text {nd }}$ and $3^{\text {rd }}$ harmonics). The Greenhouse-Geisser correction was used when sphericity was violated. For both ANOVAs, putting the "dominant eye" (the eye that the stimuli were projected into) as a between-subject factor was not significant, $F(1,35)=3.014, p=.091, \eta_{p}^{2}=.079$, neither did it show an interaction with the fixed effects (ANOVA 1: $F(5.086,178.024)=0.634, p=.676, \eta_{p}^{2}=.018$; ANOVA 2, fundamental frequency $\times$ dominant eye: $F(1.653,57.841)=0.367, p=.694, \eta_{p}{ }^{2}=.010$, frequency level $\times$ dominant eye: $\left.F(2,70)=1.715, p=.188, \eta_{p}^{2}=.047\right)$, thus the factor "dominant eye" was removed from subsequent analyses.

Because we observed a considerable amount of inter-individual variability across the frequencies, we then performed mixed effects analyses, in order to examine 1) the influence of the variation between participants (random effect factor: subj) on the centered count of seen trials (dependent variable count), and 2) whether the fundamental frequency plus harmonics model (fixed effect factors: freqfund, freqhar) better described the data, comparing to simply grouping 9 frequencies into low $(3,4,5 \mathrm{~Hz})$, middle $(6,8,9.23 \mathrm{~Hz})$, and high $(10,12,15 \mathrm{~Hz})$ frequencies without considering the fundamental frequency (fixed effect 


\section{Chapter 4}

factor: freq/v). The grouping of frequencies into low/middle/high levels was done to enable the estimation of the random effect. The analysis was performed in $R$, with the packages Ime4, Ismeans, pbkrtest. The models were fitted with R's default function Im, and the function Imer in Ime4. The comparisons between models were performed as likelihood ratio tests between a full model and a reduced model removing the factor in question, with the likelihood ratio tests performed by function anova in Ime4. The pairwise comparisons between levels of fixed effects after finding the best model justified by the data were performed with the function Ismeans and pbkrtest, with the Tukey method for multiple comparison adjustments.

Apart from the inter-individual differences, the stimulus visibility may also change as the experiment progressed, and may also result from different responses across different stimuli. To further understand the role of these factors, we examined the Kendall's tau-b correlations between the outcome visibility, with the trial orders (from 1 to 360 ) to represent the time effect, the flash frequencies, and the 10 stimuli identities. The correlation analyses were performed in MATLAB R2016a (the MathWorks, Natick, MA, USA), with the function corr. Within the data of each individual participant, the no-response trials were removed before performing the correlation. To validate the time effect, we compared the number of seen trials between the first block and the last $\left(6^{\text {th }}\right)$ block, with the Friedman test. We also performed the same correlation analysis in the eye-dominance test data, correlating the visibility of each trial with the trial order (from 1 to 60 ), the face stimuli identities, and the eyes the stimuli were projected to. The resulting tau-b coefficients across all participants were then compared to 0 with one-sample t test (two-tailed, FDR corrected) to determine whether the correlations were significant for the group, and the comparisons of correlations between each other were performed by paired-samples t tests (two-tailed). In addition, we computed the eye dominance bias scores for individual participants, by dividing the absolute difference of seen trials between left and right eyes with their sum $(|L-R| /(L+R))$. The score would be 0 for perfectly balanced dominance ( 30 seen trials for each eye), and would be 1 for the most unbalanced dominance ( 30 seen trials for one eye and 0 seen trials for the other).

\section{Results}

\section{The effect of flash frequencies}

\section{Repeated-measures ANOVAs}

The repeated-measures ANOVA of 9 flash frequencies showed a significant main effect of frequency, $F(5.164,185.914)=3.095, p=.010, \eta_{p}^{2}=.079$, indicating that the average number of seen trials was different across frequencies. The test of polynomial contrasts across the 9 frequencies showed a significant linear trend, $F(1,36)=4.725, p=.036, \eta_{p}{ }^{2}=.116$, a significant quadratic trend, $F(1,36)=5.902, p=.020, \eta_{p}{ }^{2}=.141$, and a significant $6^{\text {th }}$ order trend, $F(1,36)=4.427, p=.042, \eta_{p}{ }^{2}=.110$. See Figure 1B. Contrasting the 8 frequency levels to the routinely used frequency of $10 \mathrm{~Hz}$, the numbers of seen trials under 4,6 and $8 \mathrm{~Hz}$ were significantly fewer than that of $10 \mathrm{~Hz}\left(4 \mathrm{~Hz}: F(1,36)=6.148, p=.018, \eta_{p}{ }^{2}=.146 ; 6 \mathrm{~Hz}\right.$ : 
$F(1,36)=6.262, p=.017, \eta_{p}^{2}=.148 ; 8 H z: F(1,36)=7.426, p=.010, \eta_{p}^{2}=.171$, FDR corrected), indicating that $10 \mathrm{~Hz}$ was not the optimal frequency to induce stronger suppression.

When sorting the flash frequencies by the fundamental frequencies $(3,4,5 \mathrm{~Hz})$ and their $2^{\text {nd }}$ and $3^{\text {rd }}$ harmonics, the repeated-measures ANOVA showed a significant main effect of fundamental frequency, $F(1.663,59.878)=3.812, p=.035, \eta_{p}{ }^{2}=.096$, and a significant main effect of harmonic levels, $F(2,72)=4.077, p=.021, \eta_{p}{ }^{2}=.102$. Their interaction was not significant, $F(3.337,120.127)=1.899, p=.127, \eta_{p}{ }^{2}=.050$. Both main effects showed quadratic trends, $F(1,36)=4.427, p=.042, \eta_{p}^{2}=.110, F(1,36)=4.310, p=.045, \eta_{p}^{2}=.107$.

A

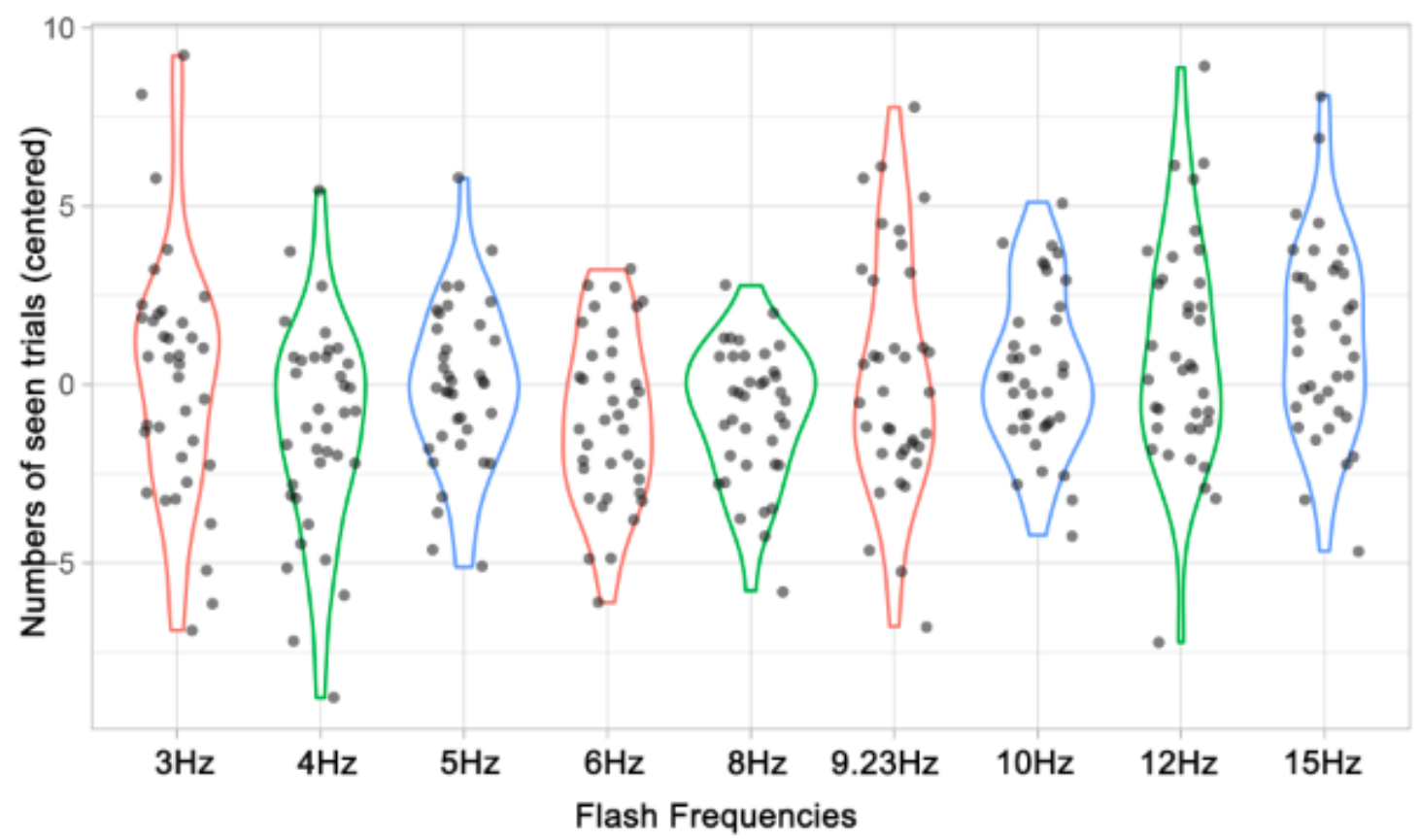

B

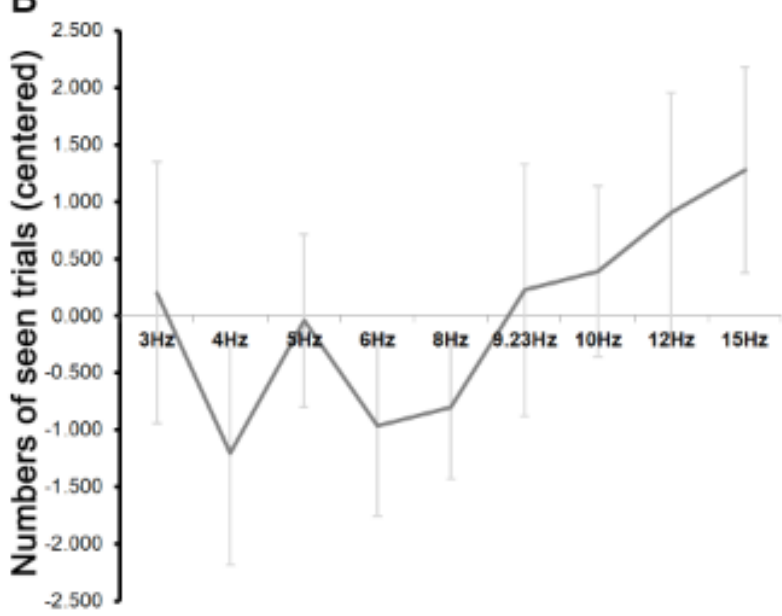

Flash Frequencies

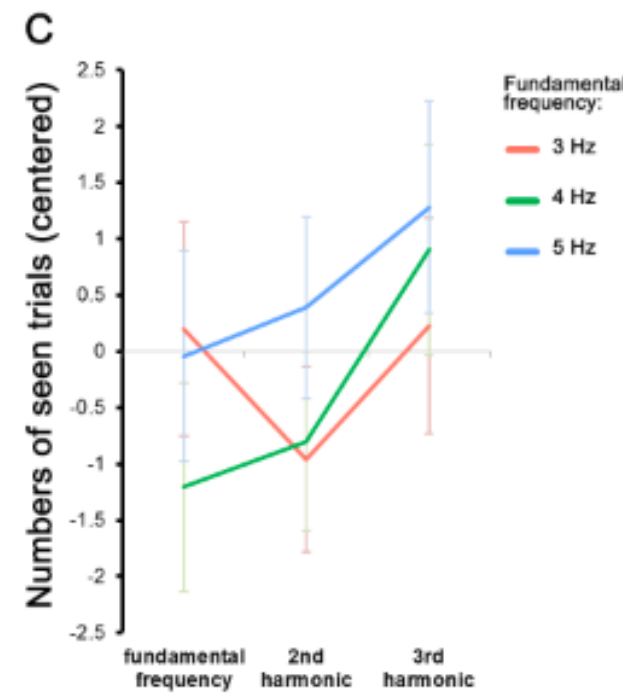

Figure 1. Numbers of seen trials per flash frequency, centered within participant by each participant's average number of seen trials across 9 frequencies. A. Raw data. The individual dots in each frequency represent how many more/less trials they consciously perceive comparing to the average across 9 frequencies, within each individual participant. For visualization purpose the dots were jittered on the $x$ axis. $B$. The average number of seen trials per frequency. Error 


\section{Chapter 4}

bars denote $95 \%$ confidence intervals. C. The average number of seen trials plotted by harmonic levels for each fundamental frequency. Error bars denote $95 \%$ confidence intervals estimated with mixed-effects analysis, after accounting for random effects of participants. See $\mathbf{C}$ for color codes of the harmonic levels.

\section{Mixed effects analysis}

In the mixed effects analysis, we constructed and compared models with different fixed and random effects. The fixed effects were to compare the fundamental flash frequencies, their harmonics and the interaction (fixed effect factors: freqfund, freqhar) with the frequencies grouped into low/middle/high frequency levels (fixed effect factor: freq/v). The random effects were to examine whether there were significant interactions of fixed effects of flash frequencies with the individual participants (random effect factor: subj). The interaction terms included simple scalar terms (1/freqlv:subj) and (1/freqfund:subj), (1/freqhar:subj), and more complex terms that included a random slope for every participant: (0+freq/v/subj), $(0+f r e q f u n d / s u b j),(0+f r e q h a r / s u b j)$. The count of seen trials centered within each participant served as the data input. Because of the centering, we did not include a random intercept for each participant (the term 1 / subj) in our models. See supplementary material for all the models examined, and the likelihood tests between them.

The final model justified by the data consisted of the fixed effects of the fundamental frequencies, their harmonics and the interaction between them, and random slopes of each participant for both fixed effects ( $\mathrm{m} 17$ in supplementary material). Comparing the full model to the one with fixed effect only showed significant random effects (m17 compared to m03), $\chi^{2}(12)=38.394, p=.0001324$, which was the inter-individual variability. Leaving out the fixed effects one by one from the full model showed that both the main effects of fundamental frequencies $\left(\chi^{2}(2)=8.9156, p=.01159\right)$, their harmonics $\left(\chi^{2}(2)=7.8124, p=.02012\right)$ and the interaction between them $\left(\chi^{2}(4)=9.6626, p=.04651\right)$ were all significant. See Figure $1 C$.

Pairwise comparisons between the fundamental frequencies showed that the $4 \mathrm{~Hz}$ frequencies had lower numbers of seen trials than the $5 \mathrm{~Hz}$ frequencies, $\mathrm{t}(36)=-2.909$, $\mathrm{p}=.0166$. Pairwise comparisons between the frequency harmonics showed a lower number of seen trials at the $2^{\text {nd }}$ harmonic than the $3^{\text {rd }}, t(36)=-2.794, p=.0220$.

\section{The time effect}

To examine the time effect that the stimulus visibility change during the progression of the experiment, a possible influence on individual differences of stimulus visibility, we performed the Kendall's tau-b correlations between visibility per trial, the flash frequencies, and the body stimuli identities in individual datasets of the main experiment. At the group level, the trial order was significantly correlated with stimulus visibility, mean tau-b coefficient $=0.211, t(36)=9.765, p=1.17 \times 10^{-11}$. The flash frequency was also significantly correlated with stimulus visibility, mean tau-b coefficient $=0.027, t(36)=2.189, p=.035$, although the coefficients were one order of magnitude smaller than that of the trial order effect, $t(36)=8.679, p=2.37 \times 10^{-10}$. The correlation of stimulus ID with the stimulus visibility 
showed a trend to significance with small coefficients, mean tau-b coefficient $=0.015$, $t(36)=1.866, p=.070$.

As a reference for the magnitude of correlation, we additionally examined the correlation of trial order to the response time (RT) for each trial. Although participants were not required to respond as fast as possible, the RT was negatively correlated with the trial order, mean tau-b coefficient $=-0.142, t(36)=-9.843, p=9.48 \times 10^{-12}$, reflecting increasingly faster $R T$ as the experiment progressed. This effect was not correlated with the trial order effect on visibility though, as the second-level correlation of these two sets of tau-b coefficients was not significant, second-level tau-b coefficient $=-0.009, p=.9482$, showing that responding more "seen" trials was not directly related to responding faster.

To confirm the time effect on visibility, we compared the numbers of seen trials between the first and the last $\left(6^{\text {th }}\right)$ block of the main experiment. The Friedman test showed that the numbers of seen trials was significantly higher for the last block than the first block, $\chi^{2}(1)=25.00, p=5.73 \times 10^{-7}$. Out of 37 participants that were included in the analysis, 33 saw more trials in the last block compared to the first one.

To check whether this time effect was already present in the eye dominance test before the main experiment, we performed the Kendall's tau-b correlation on the eye dominance test data. The trial order was again significantly correlated with the stimulus visibility, mean tau$\mathrm{b}$ coefficient $=0.122, \mathrm{t}(36)=4.152, \mathrm{p}=1.93 \times 10^{-4}$. The face stimuli identities were also significantly correlated with the stimulus visibility, mean tau-b coefficient $=0.057, t(36)=3.203$, $p=.003$, not significantly smaller than the trial order effect, $t(36)=1.762, p=.087$. The left or right eye that the stimuli were presented into were not significantly correlated with the stimulus visibility, mean tau-b coefficient $=0.014, t(36)=0.360, p=.72$.

We additionally computed the bias of the eye dominance for individual participants, which would range from 0 for perfectly balanced eye dominance, to 1 for completely unbalanced eye dominance. The mean bias score for the 37 participants was 0.130 (SD=0.164), but showed substantial variability, ranging from 0 to 0.578 , with the majority having low bias scores. See Figure 2.

\section{Discussion}

In this study we examined the effect of CFS mask flash frequency on stimulus visibility, using 9 different frequencies ranging from 3 to $15 \mathrm{~Hz}$, including $10 \mathrm{~Hz}$, which is currently the most commonly used frequency in CFS experiments. We found the number of seen trials differed across the frequencies. Additionally, we observed considerable inter-individual variability across frequencies. Controlling for this inter-individual variability using mixed-effects analysis, we found that the data were better described by a model of fundamental frequencies $(3,4,5 \mathrm{~Hz})$ and their harmonics. Both the fundamental frequencies, their harmonics and their interaction were significant, showing that the number of seen trials was lower for the $4 \mathrm{~Hz}$ frequencies compared to the $5 \mathrm{~Hz}$ frequencies, and lower for the $2^{\text {nd }}$ than the $3^{\text {rd }}$ harmonic. Examining correlations in individual data revealed that a time effect of the experiment had considerable influence on stimulus visibility: most of the participants 


\section{Chapter 4}

progressively saw more trials during the course of the experiment. This time effect was already present in the short eye-dominance test before the main experiment.
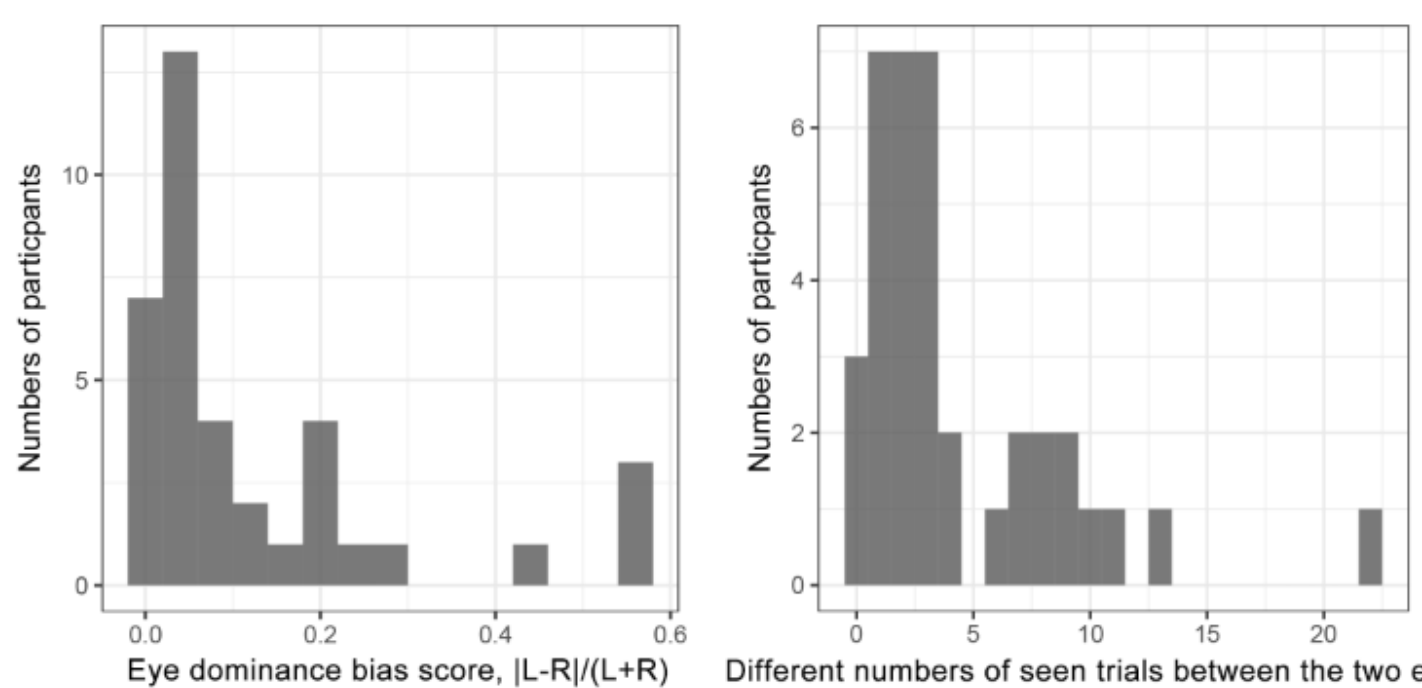

Different numbers of seen trials between the two eyes

Figure 2. A. Distribution of the eye dominance bias scores across individual participants. B. Distribution of the numbers of seen trials difference between eyes.

\section{The effect of flash frequencies on stimulus visibility}

We found that frequencies of 4,6 and $8 \mathrm{~Hz}$ showed stronger suppression than $10 \mathrm{~Hz}$ in the current sample of participants. We set out to better sample the optimal frequency range 3 $12 \mathrm{~Hz}$ suggested by Tsuchiya \& Koch (2005), which corresponded to 3.125, 6.25 and $12.5 \mathrm{~Hz}$ in their data. Comparing to the other studies on flash frequencies, our result is in agreement with Zhu et al. (2016), where they found the stimulus contrast to break from suppression peaked at around $6 \mathrm{~Hz}(5 \& 7 \mathrm{~Hz}$ in their data).

The 3 frequencies found as optimal in the current study might not be trivial, and could have functional importance for visual processing. Accumulating evidence from recent studies suggests that spatial attention on a single target is employed in the frequency range of $\sim 7 \mathrm{~Hz}$, which appeared to be an automatic process not related to a voluntary shift of attention, and this attentional resource may be divided into lower frequencies if multiple targets are present (for reviews see (Buschman \& Kastner, 2015; Fries, 2015; VanRullen, 2016; VanRullen \& Dubois, 2011). Two studies with covert spatial attention were of special interest. One study examined the visual target detection with varying target onset to the visual cue, on two objects with 3 possible locations: one was the cued location, another was on the same object of the cued location, a third was on the non-cued object. They found periodicity in the detection performance at $8 \mathrm{~Hz}$ on the cued and same-object location, and at $4 \mathrm{~Hz}$ for the different-object location. The performance of the same and different object followed an anti-phase relationship (Fiebelkorn, Saalmann, \& Kastner, 2013). The other study examined the phase differences of visually induced gamma-band activity for two targets, between hits and misses. They found that this measure was modulated at $4 \mathrm{~Hz}$ but not at other 
frequencies from 2 up to $20 \mathrm{~Hz}$, indicative of continuous attentional sampling at $8 \mathrm{~Hz}$. They also verified that this $4 \mathrm{~Hz}$ modulation was not caused by eye movements (Landau, Schreyer, van Pelt, \& Fries, 2015). The 4 and $8 \mathrm{~Hz}$ in these two studies were of the fundamental frequency of $4 \mathrm{~Hz}$.

In our experiment, we presented the targets in a single foveal location, which did not involve voluntary shifts of attention. The exact mechanism of the CFS in the brain is not yet clear, although in binocular rivalry that similarly utilizes interocular competitions, the competition sites were thought to be the lateral geniculate nucleus, V1, or a competition of inconsistent patterns in higher-level areas (Tong, Meng, \& Blake, 2006). A previous study comparing CFS to both flash suppression and binocular rivalry showed that, the suppression strength of CFS was due to multiple flashes built up along the temporal dimension, rather than a summation of flash suppression and binocular rivalry (Tsuchiya et al., 2006). Relating to the research of rhythmic attention, the stronger suppression effect we found at 4 and $8 \mathrm{~Hz}$ may thus reflect an interaction of the CFS mask to the attentional sampling mechanism, with the multiple colourful rectangles optimally occupying the attentional resources at those sampling frequencies. Our results of the mixed-effect analysis found that the fundamental frequency and harmonics model better described the data than a simple model of low/middle/high frequency levels, thus was consistent to the observation that the attention samples the visual scene at specific fundamental frequencies (specifically in the fundamental frequency of $4 \mathrm{~Hz}$ ). The frequencies involved in perceptual rhythms were so far mainly found below 15 $\mathrm{Hz}$ (VanRullen, 2016). From our data the $3^{\text {rd }}$ harmonic of $4 \mathrm{~Hz}(12 \mathrm{~Hz})$ did not seem to have a strong suppression effect. Thus it is not yet clear whether the fundamental frequency and their harmonics are important properties of attentional sampling mechanisms, or only a few frequencies around the theta band ( $7 \mathrm{~Hz}$ ) matters (VanRullen, 2016). When stimuli are consciously perceived, long-distance power and phase synchrony has been found in the beta and gamma bands (Dehaene \& Changeux, 2011). Because the CFS largely keeps stimuli from reaching visual awareness, and would occasionally allow stimuli to break from suppression, we believe its suppression impact happens at the very first bottom-up sampling stage, instead of a later stage when conscious information is globally distributed in the brain, although it would be interesting to investigate the relationships of the CFS mask frequency with the beta and gamma bands. Further research with higher temporal resolution, and/or bigger frequency ranges may help testing these hypotheses. For the stimulus, we always presented one stimulus at the center of the visual field in each trial. However, it is also worth testing whether the flash frequency would interact when multiple stimuli or multiple locations were used, as indicated by previous spatial attention findings.

For the frequencies found by Fiebelkorn et al. (2013), variability was found across participants but was relatively stable for each participant (personal communication to the authors). In our experiment, we controlled the inter-individual variability by mixed-effects analysis, but did not thoroughly examine it. Future experiments could shed more light on the variability, by linking the optimal flash frequency of each participant under CFS to other behavioural measures.

We examined the CFS mask frequency effect, using neutral bodies as target stimuli. There remains the possibility that the mask frequency shows different effect for other categories 


\section{Chapter 4}

of stimuli, and stimuli conveying additional information such as emotion. Indeed, stimuli differing in these aspects were suppressed at different durations under CFS in healthy participants (e.g. (Stein et al., 2012; Zhan et al., 2015; Zhu et al., 2016), as well as being processed differently in blindsight and neglect patients (e.g. (Tamietto et al., 2015; Van den Stock et al., 2014). However, as the suppression time for faces and bodies could be matched by manipulating the contrasts for each category (Stein et al., 2012), and similar frequency with highest suppression strength was found for both faces, houses, and symbols, despite their different suppression times, we speculate that the effect of CFS mask temporal frequency would be relatively independent to the target stimuli categories.

\section{The increase of stimulus visibility as the experiment progressed}

Our data showed a time effect: an increase of seen trials over the course of the experiment (length $50 \mathrm{~min}$ ), which could have been overlooked without examining individual data. For participants who were mostly naive to the CFS paradigm, this effect was already present in the much shorter eye dominance test $(6 \mathrm{~min})$ before the start of the main experiment. This important finding indicates that the time effect could possibly affect all CFS experiments, especially those utilizing naïve participants and stimuli that are more ecologically valid, such as faces and bodies.

Although not systematically studied before, this effect has been reported in a few CFS studies. One study mentioned it as a "learning to see" effect, showing that the hit rate under CFS grew from $36 \%$ in the first 40 trials to $64 \%$ in the last 40 trials. This effect was found together with their main research question, that hearing a valid verbal cue could facilitate the suppressed stimuli into awareness (Lupyan \& Ward, 2013). Another study adjusted the target stimuli's contrasts during the course of experiment for individual participants, and they found the contrast threshold for detecting the stimuli lowered progressively (stimuli increasingly visible) across experimental sessions (Ludwig, Sterzer, Kathmann, Franz, \& Hesselmann, 2013). A third study attributed the improvement of stimulus visibility as a training effect, and found it was generalized to stimuli of a different orientation, but not when the stimuli and masks were swapped between eyes (Mastropasqua, Tse, \& Turatto, 2015).

The increase of stimulus visibility during the experiment may relate to several causes. One possibility is that the participants may have consciously changed their decision criteria of "seeing a stimulus" as the experiment progressed. However, considering that the stimulus visibility increased in both the short and long test of our experiment, and the presence of invisible trials, it is not likely the main cause. A more likely cause may indeed be the accumulation of categorical information and the resulting expectation. Following Lupyan \& Ward (2013), a recent CFS study found the cueing effect with written words for both complex and simple visual targets, and extended this effect to the sandwich masking paradigm (Stein \& Peelen, 2015). In our experiment, seeing faces/bodies would likely act as cues themselves, to boost the stimulus visibility of subsequent trials. Other causes may be related to the fatigue and eye movements during the course of the experiment, but it needs further experimental investigations by combining CFS with eye tracking to confirm. A 
question also remains whether the increase of visibility follow the same slope for different stimulus categories.

\section{The eye dominance test}

To achieve more stable suppression of visual awareness, some previous CFS studies had presented target stimuli into the non-dominant eye, which was established by an eye dominance tests before the main experiment. The methods to determine eye dominance was not reported in all studies, and varied across those studies that did report, including the hole-in-the-card test relying on monocular viewing (e.g. (Hesselmann, Hebart, \& Malach, 2011), and tests based on binocular rivalry (e.g.(Yang, Blake, \& McDonald, 2010). However, these two measures showed low consistency with each other (Mapp, Ono, \& Barbeito, 2003; Yang et al., 2010). In our experiment, we opted for the binocular method in order to have the same measure (number of seen trials) as the main experiment. We found weak biases between eyes for most of our participants, consistent with Yang et al. (2010). The eye dominance of our participants could have been changed into the opposite by only a few trials' difference, indicating our measure was not a stable one. More stable measure could be the time for each eye that a stimulus breaks from suppression (Yang et al., 2010), although a recent study found that the imbalance of the eye dominance could be introduced by CFS presentation into one of the eyes (Kim, Kim, \& Blake, 2017), which complicates the story.

\section{Conclusion}

In summary, the current experiment found that the flash frequency of the CFS masks influenced stimuli visibility, with higher suppression strength at 4,6 , and $8 \mathrm{~Hz}$, instead of the routinely used $10 \mathrm{~Hz}$. These frequencies corresponded to the sampling frequency of spatial attention. In addition, we also observed significant inter-individual variability and an increase of visible trials as the experiment progressed. Future CFS studies need to take these factors into consideration to ensure maximal generalizability of results obtained with the CFS paradigm, and may benefit from using a flash frequency of $8 \mathrm{~Hz}$. 


\section{Chapter 4}

\section{Supplementary material}

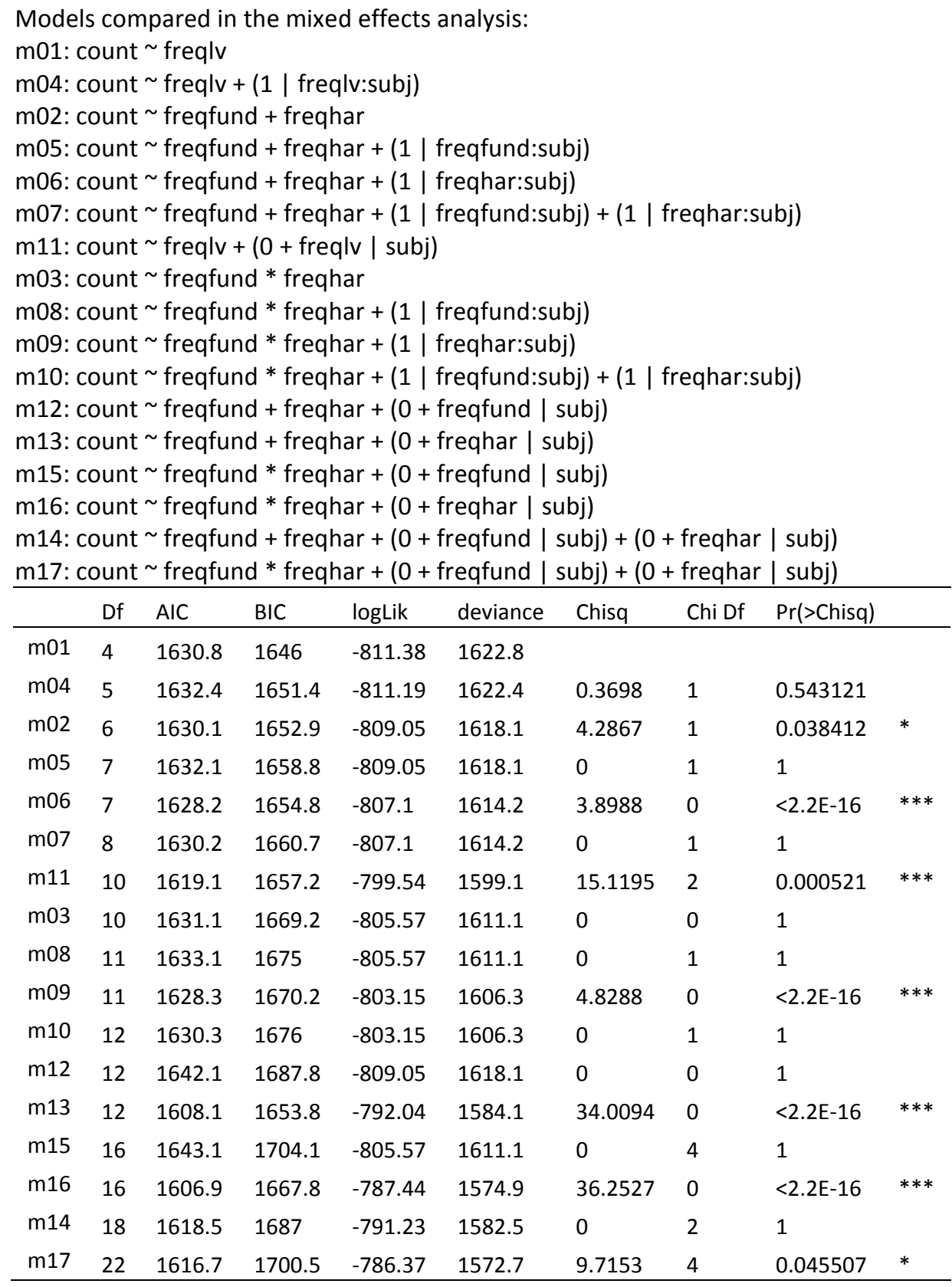

Significance codes: $0:^{1 * * * 1}, 0.001:^{1 * * 1}, 0.01:^{1 * 1}, 0.05:$ '.' $^{\prime}$

\section{References}

Brainard, D. H. (1997). The psychophysics toolbox. Spatial vision, 10, 433-436.

Buschman, T. J., \& Kastner, S. (2015). From Behavior to Neural Dynamics: An Integrated Theory of Attention. Neuron, 88(1), 127-144. doi: 10.1016/j.neuron.2015.09.017

Celeghin, A., de Gelder, B., \& Tamietto, M. (2015). From affective blindsight to emotional consciousness. Conscious Cogn, 36, 414-425. doi: 10.1016/j.concog.2015.05.007

Costello, P., Jiang, Y., Baartman, B., McGlennen, K., \& He, S. (2009). Semantic and subword priming during binocular suppression. Conscious Cogn, 18(2), 375-382. doi:

10.1016/j.concog.2009.02.003 
de Gelder, B., Vroomen, J., Pourtois, G., \& Weiskrantz, L. (1999). Non-conscious recognition of affect in the absence of striate cortex. Neuroreport, 10(18), 3759-3763.

Dehaene, S., \& Changeux, J. P. (2011). Experimental and theoretical approaches to conscious processing. Neuron, 70(2), 200-227. doi: 10.1016/j.neuron.2011.03.018

Drewes, J., Zhu, W., \& Melcher, D. (2018). The edge of awareness: Mask spatial density, but not color, determines optimal temporal frequency for continuous flash suppression. J Vis, 18(1), 12. doi: 10.1167/18.1.12

Farah, M. J. (2004). Visual agnosia: MIT press.

Fiebelkorn, I. C., Saalmann, Y. B., \& Kastner, S. (2013). Rhythmic sampling within and between objects despite sustained attention at a cued location. Curr Biol, 23(24), 2553-2558. doi: 10.1016/j.cub.2013.10.063

Fries, P. (2015). Rhythms for Cognition: Communication through Coherence. Neuron, 88(1), 220-235. doi: 10.1016/j.neuron.2015.09.034

Getov, S., Kanai, R., Bahrami, B., \& Rees, G. (2015). Human brain structure predicts individual differences in preconscious evaluation of facial dominance and trustworthiness. Soc Cogn Affect Neurosci, 10(5), 690-699. doi: 10.1093/scan/nsu103

Gray, K. L., Adams, W. J., Hedger, N., Newton, K. E., \& Garner, M. (2013). Faces and awareness: lowlevel, not emotional factors determine perceptual dominance. Emotion, 13(3), 537-544. doi: $10.1037 / \mathrm{a} 0031403$

Han, S., Lunghi, C., \& Alais, D. (2016). The temporal frequency tuning of continuous flash suppression reveals peak suppression at very low frequencies. Sci Rep, 6, 35723. doi: 10.1038/srep35723

Hesselmann, G., Hebart, M., \& Malach, R. (2011). Differential BOLD activity associated with subjective and objective reports during "blindsight" in normal observers. J Neurosci, 31(36), 1293612944. doi: 10.1523/JNEUROSCI.1556-11.2011

Jakobson, L., Archibald, Y., Carey, D., \& Goodale, M. A. (1991). A kinematic analysis of reaching and grasping movements in a patient recovering from optic ataxia. Neuropsychologia, 29(8), 803809.

Jiang, Y., \& He, S. (2006). Cortical responses to invisible faces: dissociating subsystems for facialinformation processing. Curr Biol, 16(20), 2023-2029. doi: 10.1016/j.cub.2006.08.084

Kaunitz, L. N., Fracasso, A., Skujevskis, M., \& Melcher, D. (2014). Waves of visibility: probing the depth of inter-ocular suppression with transient and sustained targets. Front Psychol, 5, 804. doi: 10.3389/fpsyg.2014.00804

Kim, H. W., Kim, C. Y., \& Blake, R. (2017). Monocular Perceptual Deprivation from Interocular Suppression Temporarily Imbalances Ocular Dominance. Curr Biol, 27(6), 884-889. doi: 10.1016/j.cub.2017.01.063

Landau, A. N., Schreyer, H. M., van Pelt, S., \& Fries, P. (2015). Distributed Attention Is Implemented through Theta-Rhythmic Gamma Modulation. Curr Biol, 25(17), 2332-2337. doi: 10.1016/j.cub.2015.07.048

Langner, O., Dotsch, R., Bijlstra, G., Wigboldus, D. H., Hawk, S. T., \& van Knippenberg, A. (2010). Presentation and validation of the Radboud Faces Database. Cognition and Emotion, 24(8), 1377-1388.

Ludwig, K., Sterzer, P., Kathmann, N., Franz, V. H., \& Hesselmann, G. (2013). Learning to detect but not to grasp suppressed visual stimuli. Neuropsychologia, 51(13), 2930-2938. doi: 10.1016/j.neuropsychologia.2013.09.035

Lupyan, G., \& Ward, E. J. (2013). Language can boost otherwise unseen objects into visual awareness. Proc Natl Acad Sci U S A, 110(35), 14196-14201. doi: 10.1073/pnas.1303312110

Mapp, A. P., Ono, H., \& Barbeito, R. (2003). What does the dominant eye dominate? A brief and somewhat contentious review. Percept Psychophys, 65(2), 310-317. 


\section{Chapter 4}

Mastropasqua, T., Tse, P. U., \& Turatto, M. (2015). Learning of monocular information facilitates breakthrough to awareness during interocular suppression. Atten Percept Psychophys, 77(3), 790-803. doi: 10.3758/s13414-015-0839-z

Milner, A. D., \& Goodale, M. A. (2006). The visual brain in action: Oxford University Press.

Pelli, D. G. (1997). The VideoToolbox software for visual psychophysics: Transforming numbers into movies. Spatial vision, 10(4), 437-442.

Schurger, A. (2009). A very inexpensive MRI-compatible method for dichoptic visual stimulation. $J$ Neurosci Methods, 177(1), 199-202.

Stein, T., \& Peelen, M. V. (2015). Content-specific expectations enhance stimulus detectability by increasing perceptual sensitivity. J Exp Psychol Gen, 144(6), 1089-1104. doi: 10.1037/xge0000109

Stein, T., Seymour, K., Hebart, M. N., \& Sterzer, P. (2014). Rapid fear detection relies on high spatial frequencies. Psychol Sci, 25(2), 566-574. doi: 10.1177/0956797613512509

Stein, T., Sterzer, P., \& Peelen, M. V. (2012). Privileged detection of conspecifics: evidence from inversion effects during continuous flash suppression. Cognition, 125(1), 64-79. doi: 10.1016/j.cognition.2012.06.005

Stienen, B. M., \& de Gelder, B. (2011). Fear detection and visual awareness in perceiving bodily expressions. Emotion, 11(5), 1182-1189. doi: 10.1037/a0024032

Tamietto, M., Cauda, F., Celeghin, A., Diano, M., Costa, T., Cossa, F. M., . . . de Gelder, B. (2015). Once you feel it, you see it: Insula and sensory-motor contribution to visual awareness for fearful bodies in parietal neglect. Cortex, 62, 56-72. doi: 10.1016/j.cortex.2014.10.009

Tong, F., Meng, M., \& Blake, R. (2006). Neural bases of binocular rivalry. Trends Cogn Sci, 10(11), $502-$ 511. doi: 10.1016/j.tics.2006.09.003

Tsuchiya, N., \& Koch, C. (2005). Continuous flash suppression reduces negative afterimages. Nat Neurosci, 8(8), 1096-1101. doi: 10.1038/nn1500

Tsuchiya, N., Koch, C., Gilroy, L. A., \& Blake, R. (2006). Depth of interocular suppression associated with continuous flash suppression, flash suppression, and binocular rivalry. J Vis, 6(10), 10681078. doi: $10.1167 / 6.10 .6$

Van den Stock, J., Tamietto, M., Hervais-Adelman, A., Pegna, A. J., \& de Gelder, B. (2013). Body Recognition in a Patient with Bilateral Primary Visual Cortex LesionsCorrespondence. Biol Psychiatry. doi: 10.1016/j.biopsych.2013.06.023

Van den Stock, J., Tamietto, M., Zhan, M., Heinecke, A., Hervais-Adelman, A., Legrand, L. B., . . . de Gelder, B. (2014). Neural correlates of body and face perception following bilateral destruction of the primary visual cortices. Front Behav Neurosci, 8, 30. doi: 10.3389/fnbeh.2014.00030

VanRullen, R. (2016). Perceptual Cycles. Trends Cogn Sci, 20(10), 723-735. doi: 10.1016/j.tics.2016.07.006

VanRullen, R., \& Dubois, J. (2011). The psychophysics of brain rhythms. Front Psychol, 2, 203. doi: 10.3389/fpsyg.2011.00203

Weiskrantz, L. (1986). Blindsight: A case study and implications.

Yamashiro, H., Yamamoto, H., Mano, H., Umeda, M., Higuchi, T., \& Saiki, J. (2014). Activity in early visual areas predicts interindividual differences in binocular rivalry dynamics. J Neurophysiol, 111(6), 1190-1202. doi: 10.1152/jn.00509.2013

Yang, E., \& Blake, R. (2012). Deconstructing continuous flash suppression. J Vis, 12(3), 8. doi: 10.1167/12.3.8

Yang, E., Blake, R., \& McDonald, J. E., 2nd. (2010). A new interocular suppression technique for measuring sensory eye dominance. Invest Ophthalmol Vis Sci, 51(1), 588-593. doi: 10.1167/iovs.08-3076 


\section{Temporal frequencies of the CFS masks}

Yang, E., Zald, D. H., \& Blake, R. (2007). Fearful expressions gain preferential access to awareness during continuous flash suppression. Emotion, 7(4), 882-886. doi: 10.1037/1528-

3542.7.4.882

Zhan, M., Hortensius, R., \& de Gelder, B. (2015). The Body as a Tool for Anger Awareness-Differential Effects of Angry Facial and Bodily Expressions on Suppression from Awareness. PLoS One, 10(10), e0139768.

Zhu, W., Drewes, J., \& Melcher, D. (2016). Time for Awareness: The Influence of Temporal Properties of the Mask on Continuous Flash Suppression Effectiveness. PLoS One, 11(7), e0159206. doi: 10.1371/journal.pone.0159206 
Chapter 4 


\section{Chapter 5}

\section{Ventral and dorsal pathways differently related to visual awareness of body postures under continuous flash suppression}

Corresponding manuscript:

Zhan, M., Goebel, R., \& de Gelder, B. (2018). Ventral and Dorsal Pathways Differently Related to Visual Awareness of Body Postures under Continuous Flash Suppression. eNeuro, ENEURO. 0285-0217.2017 


\title{
Chapter 5
}

\begin{abstract}
Visual perception includes ventral and dorsal stream processes. However, it is still unclear whether the former is predominantly related to conscious and the latter to nonconscious visual perception as argued in the literature. In this study upright and inverted body postures were rendered either visible or invisible under continuous flash suppression (CFS), while brain activity of human participants was measured with functional MRI (fMRI). Activity in the ventral body-sensitive areas was higher during visible conditions. In comparison, activity in the posterior part of the bilateral intraparietal sulcus (IPS) showed a significant interaction of stimulus orientation and visibility. Our results provide evidence that dorsal stream areas are less associated with visual awareness.
\end{abstract}




\section{Ventral and dorsal activity for body postures under CFS}

\section{Introduction}

The occipito-temporal and parietal lobes of the human brain contain two major processing streams: the ventral stream is involved more in processes related to object recognition, and the dorsal one more in spatial processing, attention, and online control of actions (Milner \& Goodale, 2006).

An important open question concerns the relation of these two processing streams to subjective awareness. A dissociation has been shown in patients with brain lesions: a patient with lateral occipital cortex damage could perform visually guided actions according to the size, shape or orientation of objects and tools, despite being unable to consciously differentiate those properties (Carey, Harvey, \& Milner, 1996; Milner, 2012); patients with parieto-temporal cortex damage (McIntosh et al., 2004) or bilateral V1 damage (de Gelder et al., 2008) showed obstacle avoidance without being consciously aware of the obstacle. Addressing the relationship between the two streams in neurotypical participants requires controlled presentation of subjectively unseen stimuli, as can be achieved using the continuous flash suppression (CFS) method. Under CFS, the dichoptic presentation of a target stimulus and a dynamic noise pattern renders the target invisible for several seconds (Tsuchiya \& Koch, 2005; Tsuchiya, Koch, Gilroy, \& Blake, 2006; Yang, Brascamp, Kang, \& Blake, 2014).

The two-stream view does not imply an absolute division, and processing of some object categories clearly involves both streams. For example, tools (Culham, Cavina-Pratesi, \& Singhal, 2006; Johnson-Frey, 2004) trigger activity related to the object category in ventral areas, but also to action-observation-execution in dorsal areas. Several functional MRI (fMRI) studies directly compared ventral and dorsal activity and their relationship with visual awareness using CFS. They varied in experimental designs, but all used stimuli of either tools or together with faces. For the ventral stream, these studies consistently showed that the activity in ventral-lateral areas including the fusiform area and the lateral occipital area covaried with subjective perceptual awareness for both faces and tools, and that the activity for invisible faces/tools was significantly lower than visible ones (Fang \& He, 2005; Hesselmann, Hebart, \& Malach, 2011; Hesselmann \& Malach, 2011; Ludwig, Kathmann, Sterzer, \& Hesselmann, 2015).

For the dorsal stream however, the evidence is still not conclusive. One of the four abovementioned studies presented visible and invisible trials in separate runs without trialby-trial subjective reports, and used long baseline conditions (Fang \& He, 2005). They found that activity in dorsal areas diminished for invisible faces but not for invisible tools, showing a dissociation of activity to the percept, and to the object categories which may differently link to the function of reaching and grasping in the dorsal stream. In comparison, the other three studies presented visible and invisible trials in the same run, with trial-by-trial subjective reports. They all found higher activity for visible tools in both ventral and dorsal areas that co-varied with the visual awareness (Hesselmann et al., 2011; Hesselmann \& Malach, 2011; Ludwig et al., 2015). In two of them that performed multivariate pattern analyses between faces and tools, Hesselmann et al. (2011) found that invisible faces and tools were only decodable in the fusiform area, although Ludwig et al. (2015) found them 


\section{Chapter 5}

decodable both in the right V3a/V7 in the dorsal stream, and in FFA in the ventral stream. A fifth study did not examine the amplitude of activity across the two streams, but specifically examined the decodability of faces and tools, with 5 different strengths of CFS masks. They found that the faces and tools were decodable in both streams with the no-mask condition and the weaker masks, associated with higher levels of subjective visibility, but were not decodable for the stronger masks with lower levels of subjective visibility (Ludwig, Sterzer, Kathmann, \& Hesselmann, 2016).

Here we examined whether the assumed dissociation between the ventral and dorsal streams holds, and whether the ventral stream is mainly related to conscious and the dorsal stream is to non-conscious perception, with stimuli of whole-body images. Human body stimuli have a unique combination of properties and are particularly useful to explore this issue, because they activate the action-related dorsal network (Rizzolatti \& Sinigaglia, 2010) similarly to tool stimuli, and at the same time, bodies are processed in category specific areas (extrastriate body area, EBA; fusiform body area, FBA) in the ventral stream (Peelen \& Downing, 2007). It has been shown that information of the body stimuli could be processed without visual awareness, through pathways other than V1, as has been found activating the EBA of the same patient with bilateral V1 lesions who showed object avoidance (Van den Stock et al., 2014). As a biologically meaningful category, the processing of bodies is also disrupted by the inversion of stimuli (Reed, Stone, Bozova, \& Tanaka, 2003) similar to faces, and under the breaking continuous flash suppression paradigm (b-CFS) which measures suppression time of stimuli and indirectly reflects the non-conscious processing, inverted bodies have been shown to be suppressed longer than upright bodies (Stein, Sterzer, \& Peelen, 2012).

We presented the body stimuli either upright or inverted, and rendered them either invisible or visible using CFS, using a slow event-related design. By including inverted versions of exactly the same upright stimuli in the current study, the experimental design enabled us to examine the interaction of body orientation and subjective visibility, and to clarify the relationship between dorsal and ventral areas with respect to visual awareness. We measured blood-oxygenation level dependent (BOLD) activity with $\mathrm{fMRI}$ of relatively high resolution $\left(2 \times 2 \times 2 \mathrm{~mm}^{3}\right)$, while participants passively viewed dichoptic stimuli through a pair of prism glasses. The four types of trials (orientation (upright, inverted) $\times$ visibility (visible, invisible)) were balanced and presented within the same runs (Figure 1). We examined the relationships between orientation and visibility both with the general linear model (GLM) analysis and ANOVA in the whole brain, and performed the ANOVAs in ventral and dorsal regions of interest (ROIs) defined with a separate functional localizer for individual participants. 
A

\section{Orientation}

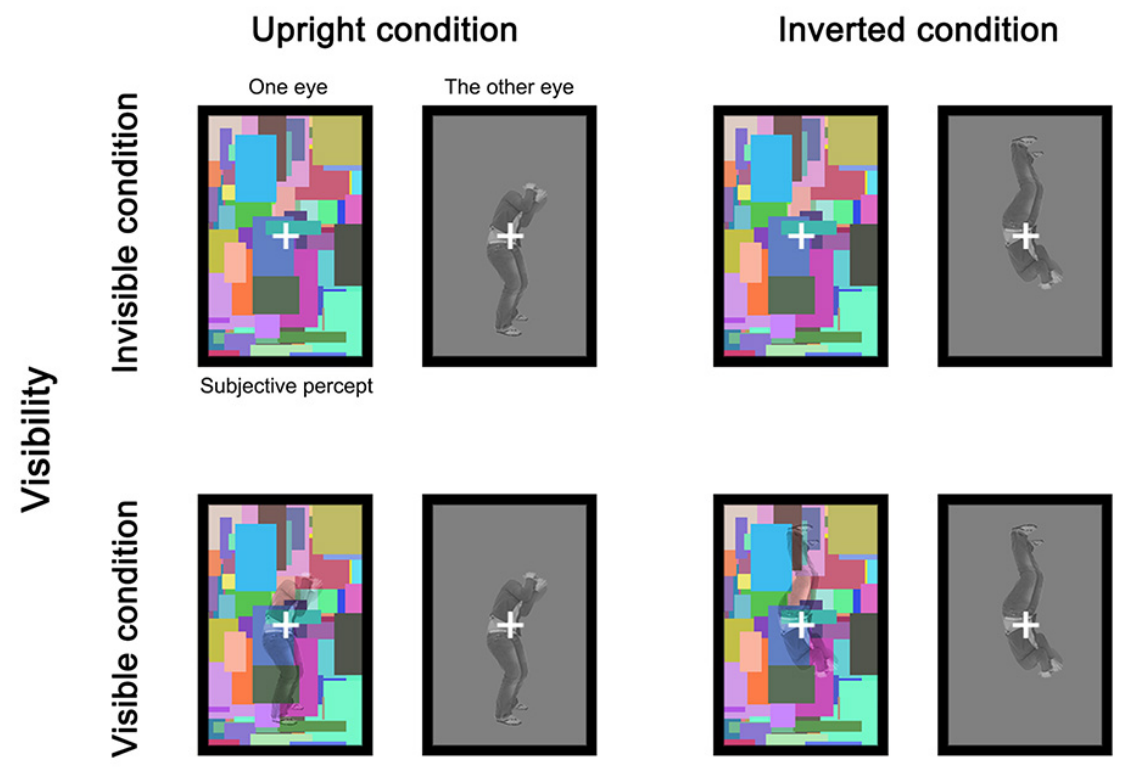

B

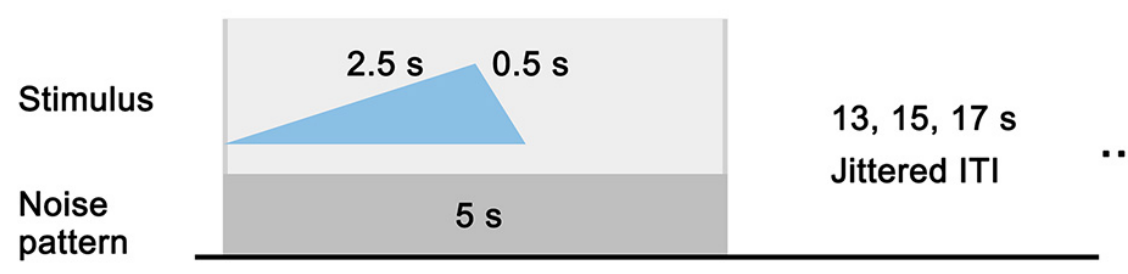

Figure 1. Stimulus presentation conditions and trial structure. A. Four stimulus conditions of the factors visibility (visible, invisible) and orientation (upright, inverted). In each condition a dynamic color noise pattern was presented in one eye, and the target stimulus was presented in the other eye. Participants could only subjectively perceive the contents in the rectangle with the dynamic noise. For visible conditions, the noise pattern was overlaid with the body stimulus. The dynamic noise was present in all conditions. B. Structure of a single trial. The target stimulus was faded in for $2.5 \mathrm{~s}$, and then faded out for $0.5 \mathrm{~s}$. The contrast of the noise pattern was constant, and the noise pattern was present for another $2 \mathrm{~s}$ after the stimulus disappeared, to avoid perceiving afterimages. A jittered inter-trial interval (ITI) followed the noise presentation. Gray vertical lines indicate the onset and offset of the stimulus presentation in a trial.

\section{Materials and methods}

\section{Participants}

Eight participants took part in the current study, the data of 7 were used for the analysis ( 2 males, mean age $=25.7, S D=4.1$, 2 left-handed), the other participant (participant 8 )'s data were excluded due to imperfect suppression (see the Validation of the suppression part in this section). As the current experiment involved long scan sessions (2 hours) thus perfect CFS suppression is crucial, we applied stringent recruiting criteria on suppression effects for 


\section{Chapter 5}

participants. Participants 2, 4 and 5 were recruited based on their performance in a separate CFS priming experiment using faces and bodies as prime stimuli where they did not perceive either faces or bodies ( $A$ total of 25 participants took part in this experiment at the time of invitation for the current fMRI study, for 16 of which the percept of the body stimuli was well suppressed, and for 9 within the 16 the percept of the face stimuli was also suppressed). Participants 1, 3, 6 and 7 were colleagues in the department that had participated in various similar CFS pilot experiments with either suppressed faces or bodies. Participant 8 was recruited from another CFS experiment (20 participants in that experiment in total), where the visibility of body and object stimuli was manipulated in the same way as in the current fMRI experiment. For participant 8 the body stimuli were suppressed in $90.5 \%$ unseen trials in that experiment. This screening procedure also precluded any noticeable training effect of the task during the current $\mathrm{fMRI}$ experiment. As it was difficult to find participants with strong and stable suppression, so we also included left-handed participants. Although all participants were familiar with the CFS paradigm, they were all naive to the aim of the current study investigating the differences of ventral and dorsal brain activity, and the interactions of stimuli orientation and visibility. The participants had no history of neurological disorders, had normal or corrected-to-normal visual acuity, and had normal stereoscopic color vision. They provided written informed consents for participation and received monetary rewards. The experimental procedures were approved by the ethical committee of Maastricht University, and were conducted in accordance with the standards established by the Declaration of Helsinki.

\section{The main experiment}

\section{Stimuli}

Images of upright body postures expressing fear (24 identities, 12 were females) were selected from a validated set of whole-body stimuli (Stienen \& de Gelder, 2011). To ensure successful suppression of stimuli during the scanning sessions, we used fearful body postures, because it was found in a previous behavioral study that fearful bodies were suppressed longer under CFS than neutral and angry bodies (Zhan, Hortensius, \& de Gelder, 2015). The bodies were aligned at the shoe level, with facial information removed, and imbedded in a gray background ( $R G B$ value $=128,128,128$, size $=240 \times 160$ pixels, $3.81^{\circ} \times 2.54^{\circ}$ of visual angles). The size of the body postures images had a height within 161 pixels $\left(2.56^{\circ}\right)$, and a width within 80 pixels $\left(1.27^{\circ}\right)$. The inverted body stimuli were created by flipping the upright stimuli upside-down. For catch trials, the stimuli image contained 1-4 dots, randomly dispersed in the image.

Dynamic Mondrian noise images with same size as the body stimuli were presented at $10 \mathrm{~Hz}$, to achieve the suppression effect. The noise images contained colorful small rectangles (with height and width within $2^{\circ}$ ) that overlapped with each other. 600 unique noise images were created, and the images presented in each trial were randomly selected from this pool.

\section{Setup for dichoptic presentation}

The dichoptic presentation both inside and outside the scanner was achieved by viewing through a pair of prism glasses. Stimuli presentation was realized in MATLAB (the MathWorks, Natick, MA, USA) with Psychtoolbox (Brainard, 1997; Pelli, 1997). The dichoptic 
stimuli were presented into two rectangles $(240 \times 160$ pixels) side by side, their centers displaced with equal distance to the center of the screen (792 pixels between centers of two rectangles, $12.41^{\circ}$ ). A frame of 10 pixels delineated the border of the rectangles, and a black fixation cross was placed in the center of each rectangle. A cardboard divider was positioned between the participant and the screen, dividing the distance between the 2 rectangles equally, to make sure that each eye of the participant only saw the rectangle ipsilateral to that eye. The diopters of the prism glasses were calculated and chosen according to the visual angles between the rectangles (Schurger, 2009). When viewing under this setup, the displacement for each rectangle would be removed by the prism glasses, thus shifting both of the rectangles back to the center of the screen. Participants were asked to free-fuse the two rectangles into one, utilizing the frame and fixation cross of each rectangle. Upon successful fusion, participants would perceive a tunnel-like view, with the divider showing up on either side as the wall of the tunnel, and with one rectangle at the end of the tunnel in the center of the screen. Since the width of the perceived tunnel depended on the distance between the rectangles, to ensure the horizontal field of view of the gray background in the scanner was not too narrow for the participants, and for practical reasons (we have only one pair of prism glasses for each diopter), we used prism glasses of a bigger diopter (diopter=12 for each eye) in the scanner, and a smaller diopter (diopter=8 for each eye) outside the scanner. Apart from the distance between the two rectangles, other parameters of the experiment were kept the same both inside and outside the scanner. Participants reported no difficulty in merging the two rectangles into one, either inside or outside the scanner.

\section{Procedure of the main experiment}

The main experiment was of a slow event-related design. In each trial, a stimulus image was projected into one of the rectangular frames, and the dynamic noise was simultaneously projected into the other frame. The stimulus image was faded in from $0 \%$ to $50 \%$ contrast in $2.5 \mathrm{~s}$, and subsequently faded out to $0 \%$ contrast in $0.5 \mathrm{~s}$. The dynamic noise was presented at full contrast. To eliminate any possible afterimages of the stimulus, the dynamic noise was kept on the screen for another $2 \mathrm{~s}$ after the stimulus faded out. Each trial was followed by an interval of 13,15 , or $17 \mathrm{~s}$. The fixation cross changed to white at $1 \mathrm{~s}$ before the start of each trial, remained white through the trial, and changed to black at the inter-trial interval (ITI).

The experiment was of a $2 \times 2$ factorial design, where stimuli were presented upright or inverted (orientation), invisible or visible (visibility). To keep the visible and invisible conditions as close as possible, the visible trials were created by overlaying the stimuli onto the noise pattern, resulting in a subjective percept of the body stimuli fading in and out with the presence of the noise pattern by all participants, which was a distinct percept from the invisible trials (percept of noise pattern only). To exclude confounds due to introspection and to motor response, we refrained from including a trial-by-trial report of the subjective percept.

For catch trials, the dot images were presented in both rectangles, similar to the body stimuli in the visible condition, so that the participants could see the dots fading in and out in the noise pattern. The trials were followed by a response screen for $2 \mathrm{~s}$, indicated by a white circle replacing the fixation cross. 


\section{Chapter 5}

Participants were instructed to respond only to the dot trials, where they should answer during the response screen presentation whether the number of the dots was odd or even, by pressing one of two corresponding buttons on a MR-compatible button box. For all the other trials, they were asked to fixate on the cross and passively view the presentation. The passive viewing task was to avoid any confound of response-related activation, which could be observed in the parietal and frontal areas. For illustration purposes, the big influence of the active response on the BOLD percent signal change in the current study can be seen in Figure 3 and Figure 4. Participants were also advised not to blink during the trials if possible, and to blink between the trials if needed.

The main scanning session consisted of four functional runs of $19 \mathrm{~min} 10 \mathrm{~s}$ each. Within each run were 48 target trials ( 12 per condition) and 8 catch trials, presented in pseudorandom order. The side of the eyes that the dynamic noise projected into was also randomized and counterbalanced within the whole session. In total each individual stimulus was projected into each eye twice: once visible and once invisible. For one participant, 3 functional runs were acquired; for the other participants, 4 functional runs were acquired. The anatomical scan was performed after 2 functional runs.

\section{Scanning parameters}

The scanning was carried out in a Siemens 3T Prisma whole-body scanner (Siemens, Erlangen, Germany), with a 64-element head-neck coil. In the scanner, stimuli were backprojected with a LCD projector (Panasonic PT-EZ570, screen resolution $=1920 \times 1200$, refresh rate $=60 \mathrm{~Hz}$ ) on a screen $75 \mathrm{~cm}$ away from the head of the participant. The cardboard divider was placed in the bore between the head coil and the screen. A T2*-weighted gradient echo EPI sequence was used to acquire functional data covering the whole brain, with $2 \times 2 \times 2 \mathrm{~mm}^{3}$ resolution ( 64 slices without gaps, $\mathrm{TR}=2000 \mathrm{~ms}, \mathrm{TE}=30 \mathrm{~ms}$, flip angle $=77$, simultaneous multislice acquisition acceleration factor $=2, F O V=200 \times 200$, matrix size $=100 \times 100)$. A T1-weighted MPRAGE sequence was used to acquire the anatomical structure images $\left(1 \times 1 \times 1 \mathrm{~mm}^{3}\right.$, $\mathrm{TR}=2300 \mathrm{~ms}, \mathrm{TE}=2.98 \mathrm{~ms}$ ).

\section{Validation of the suppression effect}

For each participant, the effectiveness of suppression was validated by verbal reports during the scan after each run, and by behavioral validation runs before and after the scan. We based our decision of data selection mainly on the results of the behavioral validation runs.

To obtain online estimates of the CFS suppression efficiency during scanning, participants responded to the following three questions after each run, 1) In what percentage of trials did you see something in the noise? 2) Were there any merging problems during the scan? 3) Did you see a sudden appearance of the stimulus in the noise, rather than a gradual fadingin? A run with response of more than $60 \%$ seen trials (the actual percentage would be $57 \%$ when taking the seen catch trials into account), or sudden perception of stimulus in the noise, or any merging problem, would indicate that during the scan the suppression was not working perfectly. None of the runs included for data analysis had these problems.

The behavioral validation runs were carried out immediately before and after the scan, outside the scanner. The stimuli were presented on an LCD screen (Acer VG248, 3D capable, 
resolution=1920 $\times 1080$, refresh rate $=60 \mathrm{~Hz}$ ), in a room with dim light. The distance between the two rectangles was adjusted according to the diopter of the prism glasses ( 276 pixels between centers of two rectangles, $9.15^{\circ}$, diopter $=8$ ) to render stable fusion. Trials and their order in the runs before and after the scan corresponded to the run 1 and 2 in the scanner. There was no catch trial in the validation runs; instead a response screen with a circle (same as the one in the main experiment) was presented after stimulus presentation for each trial. Participants were required to respond whether they saw anything in the noise, by pressing either 1 (seen) or 2 (unseen) on the keyboard during the response screen on a trial-by-trial basis. If a participant responded "seen" for more than 2 times for the unseen (suppressed) trials in either one of the validation runs, including trials without response, the dataset of the participant would be excluded from analyses. The data of 7 participants in this study satisfied the inclusion criterion (average accuracy for visible trials: $99.4 \%$, average accuracy for invisible trials: $96.7 \%$ ), showing that their subjective percept tightly followed our planned visibility manipulation. To further ensure that the stimuli were suppressed in the invisible trials during the $\mathrm{fMRI}$ scan, participants were asked again after the scan whether their visual experience of the stimuli was similar to that in the behavioral tasks before the scan. The percept of a stimulus escaping suppression (a stimulus suddenly appearing in the noise, instead of fading in slowly) was also clearly explained to the participants. All 7 participants reported not having such percept. The $8^{\text {th }}$ participant reported more than $70 \%$ seen trials after $3 \mathrm{fMRI}$ runs in the scanner (with catch trials, reported 65-70\%, 70-75\%, 60-70\% respectively), and responded 3 times "seen" for unseen trials in the behavioral test after scan (with no catch trials, reported percentage of seen trials: 50-60\%, actual percentage $56 \%)$. Consistent with the behavioral test, after the scan this participant reported that in the behavioral test before she saw 50\% trials (actual percentage=52\%, 1 trial breaking suppression), while reported "seeing more" in both the 2-4 runs in the scanner and the behavioral test after scan. This participant was excluded from the analysis. The decrease of stimuli suppression efficiency for this participant might be the same effect reported by a few previous CFS studies, where participants saw more stimuli as the experiment progressed (Ludwig, Sterzer, Kathmann, Franz, \& Hesselmann, 2013; Lupyan \& Ward, 2013; Mastropasqua, Tse, \& Turatto, 2015; Stein \& Peelen, 2015).

In total 26 runs ( 575 volumes each) from 7 participants were included in the analysis. One run from another participant was excluded, due to merging problems caused by a contact lenses issue happened during that run. Another participant completed 3 runs instead of 4 runs.

\section{Functional localizer}

Participants were also scanned with a functional localizer run (432 volumes) in a separate session, where they passively viewed stimuli of faces, bodies, houses, tools and words in blocks. Facial stimuli were front-view neutral faces from the Karolinska Directed Emotional Faces (Lundqvist, Flykt, \& Öhman, 1998) (24 identities, 12 males). The part below the neck (clothes, hair etc.) was removed from the face images. Body stimuli (de Gelder \& Van den Stock, 2011) were neutral still front-view bodies different from the ones used in the main experiment ( 20 identities, 10 males), with the facial information removed. House and tool images were obtained from the internet. The house images consisted of 19 facades of 


\section{Chapter 5}

houses with 2-to-3-storey height, and the tool images consisted of 18 hand-held tools. Words images consisted of high-frequency English words of 4-6 letters in Arial font. All the images were imbedded within a gray background (RGB value $=157,157,157)$, spanning a visual angle of 3.65 degrees ( 230 pixels). Each block consisted of 12 stimuli from the same category; each stimulus was presented for $800 \mathrm{~ms}$, followed by an interval of $200 \mathrm{~ms}$. An inter-block interval of $12 \mathrm{~s}$ followed each block presentation. Blocks of each category were presented 7 times, and the presentation order of the stimuli and the blocks were pseudorandomized.

\section{Data processing}

The acquired data were processed in BrainVoyager (Brain Innovation, Maastricht, Netherlands). Functional data underwent default slice scan time correction, 3D motion correction, temporal GLM with Fourier basis high-pass filtering of 2 cycles. The functional datasets were then aligned to the anatomical images, brought into Talairach space, and underwent spatial smoothing with a Gaussian filter of $4 \mathrm{~mm}$ FWHM.

\section{GLM analyses}

Random effects group analyses with General linear model (GLM) were applied to the functional data of the main experiment. Predictors for each condition were convolved with the default two-gamma hemodynamic response function. The parameters from 3D head motion correction were z-transformed and added as confound predictors into the GLM analyses. The percent signal change values for each participant were extracted for subsequent Region of interest (ROI) analyses. A $2 \times 2$ ANOVA with orientation and visibility was performed on the whole-brain basis. To observe the holistic processing of fearful bodies, contrast of upright invisible > inverted invisible was also performed. The clusters of the ANOVA and the contrast analyses were corrected for multiple comparison by cluster threshold estimation (initial threshold $p=.005$ for the ANOVA results, initial threshold $p=.01$ for the contrast results, Monte Carlo simulation $n=5000$ ).

\section{ROI analyses}

Functional ROIs were defined by GLM contrasts on the functional localizer data, individually for each participant. See Figure 2. Ventral ROIs were defined by the contrast bodies $>$ houses $(p=.001$ uncorrected). Clusters that were located in the lateral occipital sulcus were marked as EBA; clusters located in the fusiform region were marked as FBA. Dorsal ROls were defined for the anterior, middle and posterior intraparietal sulcus (IPS) bilaterally, by contrasting tools $>$ baseline ( $p=.001$ uncorrected). Spheres (radius $=4 \mathrm{~mm}$ ) were defined at the peak activation sites located in the anterior (connecting post central sulcus), middle, and posterior segments of IPS, respectively. As a comparison to the ventral and dorsal areas, sphere ROls of the primary visual cortex (V1) were defined at the occipital pole, at the spots in bilateral occipitopolar sulci where the calcarine sulci pointed to (radius $=4 \mathrm{~mm}$ ). The V1 ROIs defined anatomically were located within the extensive cluster activated by visual presentation of the 5 conditions in the functional localizer versus baseline $(p=.00001$, uncorrected). For FBA, EBA, pIPS, mIPS, aIPS, and V1, we performed a group-level ANOVA of ROI $(6$ areas) $\times$ laterality (left, right) $\times$ orientation $\times$ visibility, where for each unilateral ROI one averaged percent signal change value per participant was entered as input. This group- 
level ANOVA did not show either a significant main effect of laterality $(F(1,1)=2.453, p=.362)$, or interactions with laterality $(\mathrm{ROI} \times$ laterality: $F(5,5)=0.550, p=.736$; laterality $\times$ orientation: $F(1,1)=3.903, p=.298 ; R O I \times$ laterality $\times$ orientation: $F(5,5)=1.048, p=.480$; laterality $\times$ visibility: $F(1,1)=0.606, p=.579$; laterality $\times$ orientation $\times$ visibility: $F(1,1)=0.537, p=.597 ; R O I \times$ laterality $\times$ orientation $\times$ visibility: $F(5,5)=1.430, p=.352$ ). Thus we merged the bilateral ROI pairs into single ROls. For some of the dorsal areas, only unilateral ROIs could be defined in some participants (e.g. the right alPS could only be defined in 3 participants), in those cases the data of the unilateral ROI were entered into further analysis. To compare the ventral and dorsal ROIs directly, the bilateral FBA and EBA ROIs were merged into one combined ventral $\mathrm{ROI}$, and the bilateral ROIs along the IPS were merged into one combined dorsal ROI. The mean percent signal change values from the GLM analysis were extracted for each resulting ROI of each participant. Group-level repeated-measures ANOVAs were performed in SPSS. We first conducted an ANOVA of stream (ventral, dorsal) $\times$ orientation (upright, inverted) $\times$ visibility (visible, invisible) with the data of the combined ventral and dorsal ROIs. In the case that an interaction was present, we examined the orientation $\times$ visibility ANOVA in the specific stream, then conducted subsequent ANOVAs with the data of individual ROIs.

A
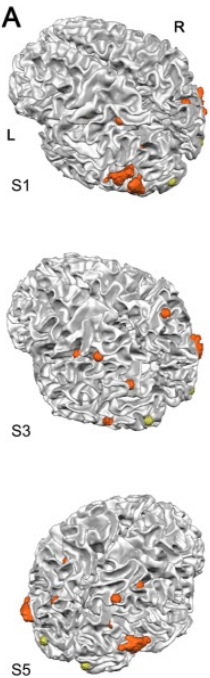

S5

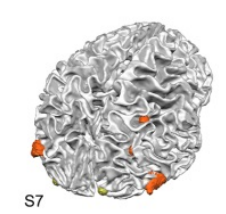

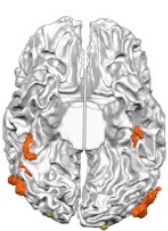
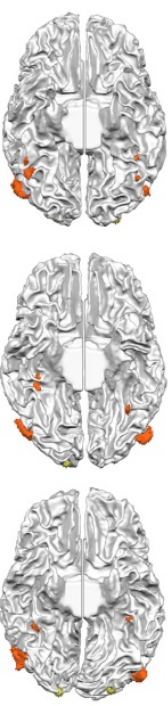
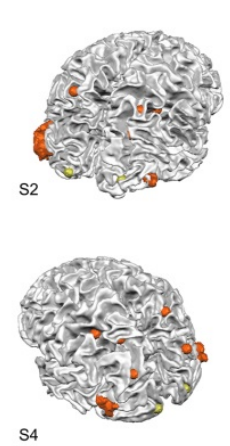

S4
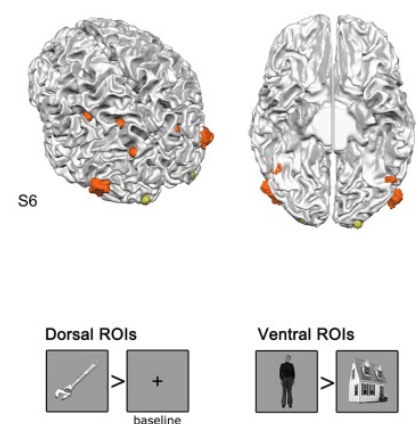
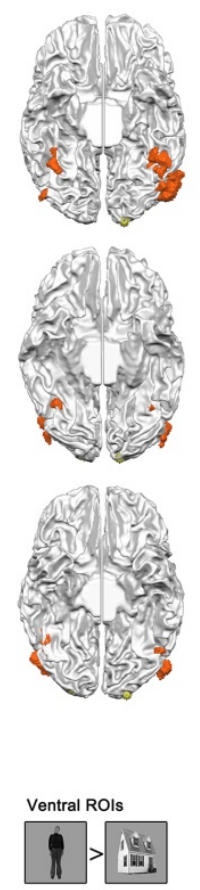

B

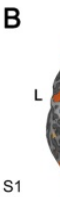

S1

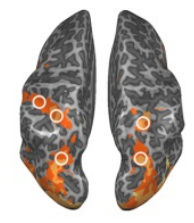

so

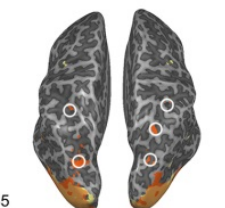

S5

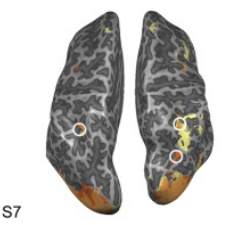

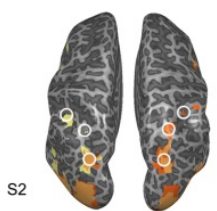
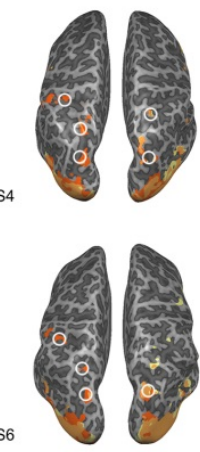

S6

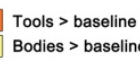

Overlap

Figure 2. Definition of ROIs in individual participants, shown in neurological view. A. Ventral, dorsal and V1 ROIs in individual participants, defined by separate functional localizer data. Ventral ROIs (orange color, irregular shape) were defined by the contrast bodies $>$ houses $(p=.001$ uncorrected); dorsal spherical ROls (orange color, spherical shape) were drawn at the peak activation sites of the contrast tools > baseline (the fixation cross, $p=.001$ uncorrected), in the posterior, middle, anterior branches of IPS respectively. V1 spherical ROIs (yellow color) were defined inside bilateral occipitopolar sulci. Participants S2 and S5 are left handed. The dorsal views are shown in the angle and side where most of the dorsal ROls could be seen. Some of the dorsal ROIs could not be defined in all participants. The ventral view shows the ventral ROls. If the clusters in either EBA or FBA consisted of multiple smaller clusters, they were grouped into 


\section{Chapter 5}

one. The example stimuli of the functional localizer and the contrasts are shown in the lower right corner. B. The areas activated by bodies and tools largely overlapped, especially in the posterior part of the IPS. Areas shown were: tools > baseline ( $p=.001$ uncorrected), bodies $>$ baseline ( $p=.001$ uncorrected). The locations for dorsal ROIs were marked with white circles.

\section{ROI analysis in individual participants}

To rule out that the observed results of group-level ANOVAs in our ROI analysis were driven by a minority of participants, we performed within-participant ROI analysis in the 7 individual participants, examining the prevalence of effects (or no effects in the dorsal stream). To be most comparable to the group-level ROI analysis, we fitted the same GLM to each run in individual participants. The percent signal changes (parameter estimates) of each condition were extracted from the same bilateral ROIs of the ROI analysis (including the combined ventral and dorsal ROIs, and the 6 individual ROIs), in individual participants, and entered into within-participant repeated-measures ANOVAs. Because the numbers of runs were different across participants, the numbers of parameter estimates included in the ANOVAs were different ( 3 estimates per condition in participants $S 1$ and S3, 4 estimates per condition in all 5 other participants). The ANOVAs included the stream $\times$ orientation $\times$ visibility ANOVA in the ventral/dorsal combined ROIs, and the orientation $\times$ visibility ANOVAs in the 6 individual ROIs. Lastly, to compare with the results obtained in Fang \& He (2005), we performed the pairwise comparisons of upright visible versus upright invisible conditions in these 8 ROls.

\section{Results}

Whole brain analysis. We conducted a whole-brain ANOVA at the group level, with orientation (upright, inverted) and visibility (visible, invisible) as factors. See Figure $\mathbf{3}$ and Table 1 for detailed cluster information. The main effect of orientation (upright, inverted) was observed in clusters mainly in the frontal lobe, and a cluster close to the EBA region defined with the functional localizer. A main effect of visibility (visible, invisible) was observed mainly in clusters in the ventral pathway, including bilateral EBA, FBA, lateral occipitotemporal cortex, and right anterior inferior temporal cortex. Clusters in the dorsal pathway were located in bilateral anterior IPS, and right middle frontal gyrus (corresponding to the frontal eye field, FEF). Other clusters were located at the right inferior frontal lobe, and right posterior cingulate sulcus.

Importantly, the interaction of visibility and orientation was observed mainly in clusters of the parietal and frontal cortex, that overlap with regions of the dorsal attention network (Corbetta \& Shulman, 2011). The parietal clusters included left medial IPS, left precuneus, right posterior IPS. The frontal clusters were located along bilateral superior frontal sulci, mostly at the location of FEF, but also more anteriorly for two clusters. Another cluster was located in the right anterior cingulate sulcus, close to the presupplementary motor area. Importantly, the interaction effect also revealed clusters in subcortical areas, including the 
left pulvinar and the right caudate nucleus. When mirrored to the right hemisphere, the coordinates of these two clusters corresponded to the focal lesion sites found in spatial neglect patients with restricted subcortical lesions (Karnath, Himmelbach, \& Rorden, 2002).

We also conducted a whole-brain contrast of upright invisible > inverted invisible, which showed clusters mainly in the frontal lobe. Importantly, a cluster was present in the right inferior occipital sulcus, showing higher activity for upright bodies. This indicates that despite being invisible, the upright bodies were nonetheless processed more extensively than the inverted ones in the ventral pathway. A cluster was also present in the right caudate nucleus.

ROI analysis. The functional localizer included still images of faces, bodies, houses, tools and words. We defined ventral ROls by the bodies $>$ houses contrast ( $p=.001$ uncorrected), leading to ROIs of bilateral EBA and FBA. Because the tools activate dorsal action observation and execution related structures, we defined the dorsal ROls by the tools $>$ baseline contrast ( $p=.001$ uncorrected). The areas activated by tools largely overlapped with those activated by bodies (bodies>baseline, $p=.001$ uncorrected), especially at the posterior IPS. See Figure $\mathbf{2}$ for the overlaps in individual participants. Sphere ROIs of $4 \mathrm{~mm}$ radius were defined at the peak activation sites in the anterior (connecting postcentral sulcus), middle, and posterior segments of IPS, respectively (labelled aIPS, mIPS, pIPS). For comparison with the ROIs in the ventral and dorsal streams, we also defined sphere ROIs in the bilateral primary visual cortex (V1) that was activated by visual presentation of these 5 stimuli categories. See Figure $\mathbf{2}$ for ROls of individual participants. Because we did not find main effects or interactions related to the laterality factor in the group-level ANOVA of areas (6 ROI pairs) $\times$ laterality (left, right) $\times$ orientation $\times$ visibility, we merged the bilateral ROIs in each area into one ROI, and then combined the ventral and dorsal ROIs respectively, to directly examine whether the dorsal stream areas indeed show a different response pattern than the ventral stream.

First, we performed a repeated-measures ANOVA of stream (ventral/dorsal) $\times$ orientation $\times$ visibility on the averaged percent signal changes of the combined ventral ROI and the combined dorsal ROI. If the response patterns differ between the two streams across the conditions, it would lead to an interaction of stream $\times$ visibility. Indeed, we found a significant interaction of stream $\times$ visibility, $F(1,6)=30.821, p=.001, \eta_{p}{ }^{2}=.837$, and a significant interaction of stream $\times$ orientation $\times$ visibility, $F(1,6)=7.307, p=.035, \eta_{p}{ }^{2}=.549$, in line with our prediction. The main effect of visibility was also significant, $F(1,6)=33.370, p=.001, \eta_{p}{ }^{2}=.848$. We subsequently performed the orientation $\times$ visibility ANOVA with the averaged activity separately for each stream. 


\section{Chapter 5}

A

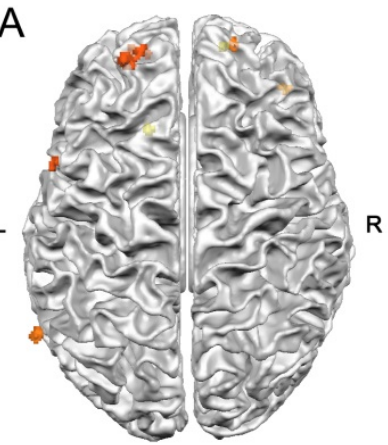

C

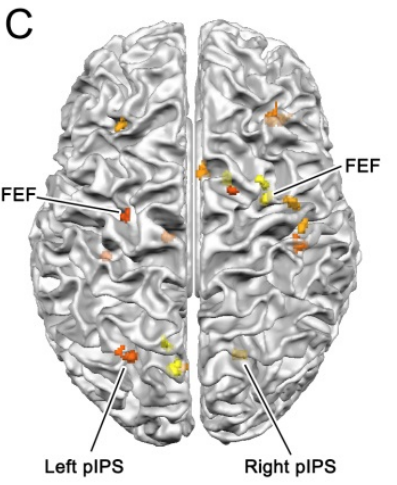

D

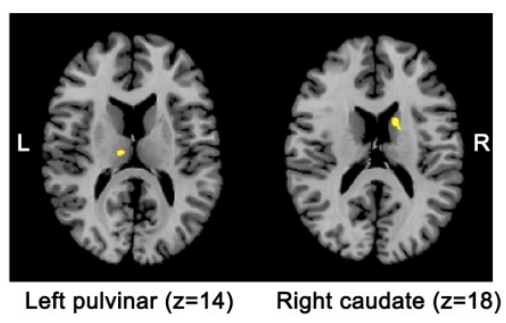

B

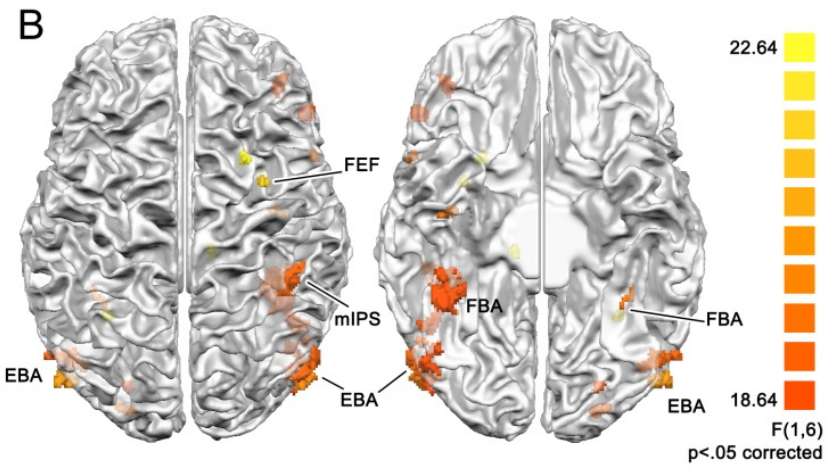

$E$
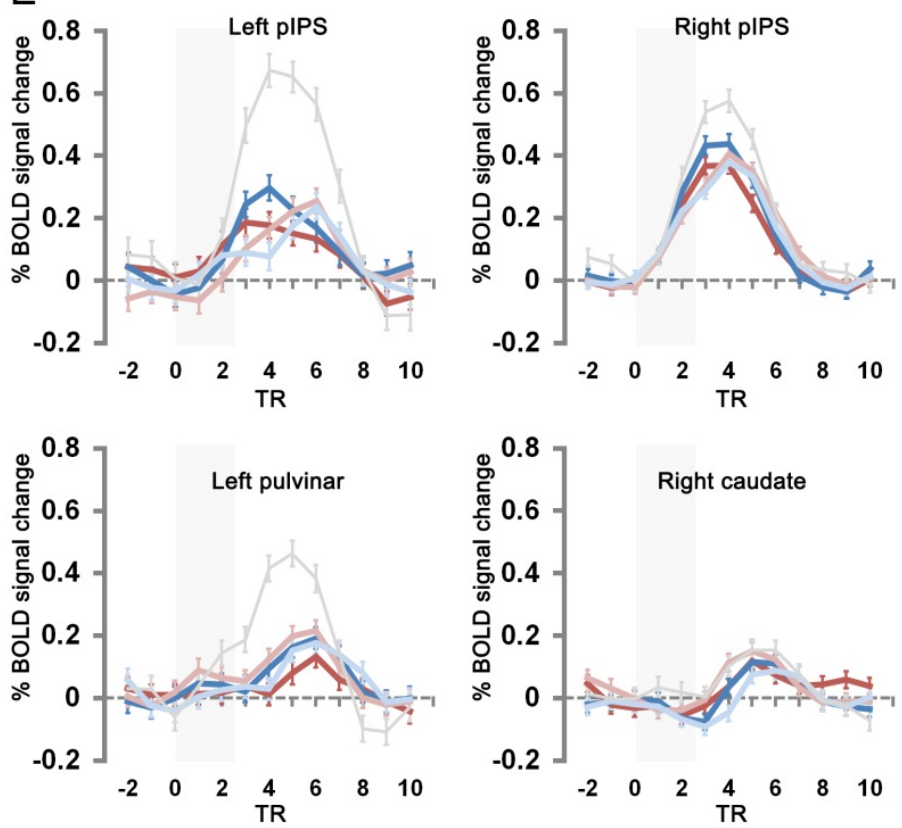

Upright visible Inverted visible

Upright invisible $\quad$ Inverted invisible $\quad$ Catch trial (visible dots)

$\mathrm{F}$
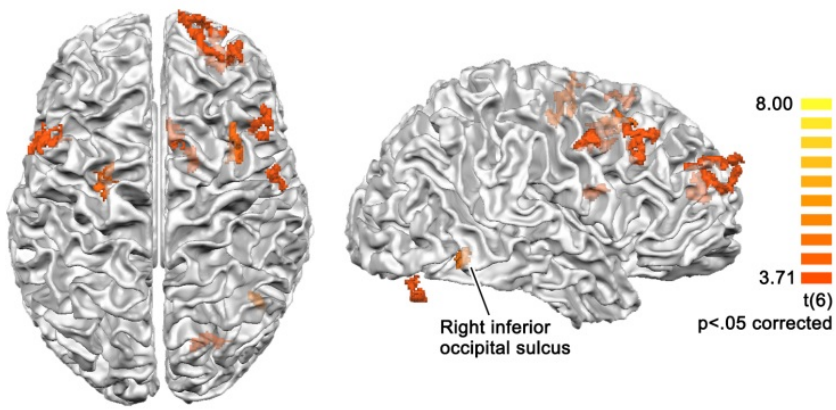

Figure 3. Clusters of the group-level whole-brain ANOVA, and contrast analysis of upright invisible > inverted invisible (cluster size corrected, initial threshold $p=.005$ for the ANOVA, $p=.01$ for the contrast, Monte Carlo simulation $n=5000$ ), projected onto the 3D surface of white-gray matter boundary of one participant, shown in neurological view. A. Clusters showing a main effect of orientation (upright, inverted). B. Clusters showing a main effect of visibility (visible, invisible). C. Clusters showing the interaction of orientation and visibility. D. The subcortical clusters from C (in-slice neurological view). The color bar for the clusters in A-D is shown in B. E. The percent signal change of the clusters in $\mathbf{C}$ and $\mathbf{D}$, for the left and right pIPS, the left pulvinar, 
and the right caudate clusters. Gray areas in the time course plots indicate the duration of dynamic noise presentation. The next trial started at TR 9-11 with a jittered ITI $(13,15$ or $17 \mathrm{~s}$ after the offset of the dynamic noise). The time courses for catch trials (visible dots, requiring participants to respond by button pressing after dynamic noise presentation) were plotted with gray thin lines, as a comparison. Note that the time courses for catch trials have higher percentsignal change than the main conditions. F. Clusters shown by the contrast upright invisible > inverted invisible. The lateral view of the brain provides a clearer view of the two posterior clusters (one in the right inferior occipital sulcus and another in the right cerebellum, shown outside the cortex mesh).

The combined ventral ROI showed strong main effects of orientation, and visibility, with no interaction effect. Similar to the ventral clusters shown by the main effect of visibility in the whole-brain ANOVA, visible bodies consistently elicited higher activity than suppressed invisible bodies, $F(1,6)=38.063, p=.001, \eta_{p}{ }^{2}=.864$, which is in accordance with the findings in CFS studies using other stimulus categories (Fang \& He, 2005; Hesselmann \& Malach, 2011; Jiang \& He, 2006; Yang et al., 2014). Upright bodies also elicited higher activity than inverted ones, $F(1,6)=16.297, p=.007, \eta_{p}{ }^{2}=.731$, also consistent with studies using other categories of inverted stimuli, such as faces (Gilaie-Dotan, Gelbard-Sagiv, \& Malach, 2010; Pinsk et al., 2009). See Table 2 for the statistical results of the ANOVA. We also examined the averaged percent signal changes for each condition in the FBA and EBA ROIs separately, see Figure 4 and Table 2. Notably, the reduced activation for inverted bodies was consistent across visibility conditions, as a main effect of orientation was found in both the FBA and EBA ROIs (FBA: $F(1,6)=9.950, p=.020, \eta_{p}{ }^{2}=.624 ; E B A: F(1,6)=13.230, p=.011, \eta_{p}{ }^{2}=.688$ ), without interaction effects to visibility. For the invisible conditions, post-hoc paired t-test showed significantly higher activity for the upright bodies in the FBA ROI $(t(6)=3.111, p=.021)$, and a trend to significance in the EBA ROI $(t(6)=2.154, p=.075)$. Together with the activation in right inferior occipital gyrus observed under the contrast upright invisible $>$ inverted invisible in the whole-brain analysis, this ROI result shows that ventral body-specific areas are sensitive to the orientation of body stimuli even when the bodies are presented without visual awareness.

In the combined dorsal ROI, the ANOVA of orientation $\times$ visibility again showed a main effect of visibility, $F(1,6)=9.172, p=.023, \eta_{p}^{2}=.605$. Important however, it also showed an interaction of orientation $\times$ visibility, $\left.F(1,6)=13.624, p=.010, \eta_{p}{ }^{2}=.694\right)$. To directly compare our results to other CFS studies without manipulation of stimulus orientation, we also performed the ANOVA stream $\times$ visibility with only the upright conditions. Again a strong interaction of stream $\times$ visibility was observed $\left(F(1,6)=34.612, p=.001, \eta_{p}{ }^{2}=.852\right)$, together with the main effect of visibility $\left(F(1,6)=24.987, p=.002, \eta_{p}{ }^{2}=.806\right)$. To better understand the interaction effects found in the dorsal stream, we performed an ANOVA of area (pIPS, mIPS, aIPS) $\times$ orientation $\times$ visibility. Again we found the interaction orientation $\times$ visibility $(F(1,5)=10.853, p=.022$, $\left.\eta_{p}{ }^{2}=.685\right)$, but we also found a significant main effect of area $(F(2,10)=9.962, p=.004$, $\left.\eta_{p}^{2}=.666\right)$, and a strong interaction of area $\times$ orientation $\times$ visibility $(F(2,10)=9.449, p=.005$, $\left.\eta_{p}{ }^{2}=.654\right)$, indicating that the response patterns changed across the areas within the dorsal 


\section{Chapter 5}

stream. The main effect of visibility showed a trend towards significance, $F(1,5)=6.149$, $\mathrm{p}=.056, \eta_{\mathrm{p}}{ }^{2}=.552$. Indeed, separate inspections of the activity in pIPS, mIPS and alPS ROIs showed that the interaction effect of orientation $\times$ visibility was present in both pIPS and mIPS ROIs, but was not present in the alPS ROI, which showed a main effect of visibility instead, with higher activity for upright than inverted bodies, similar to the pattern of the ventral areas. For the pIPS ROI, the main effect of visibility was also present. In both the pIPS and mIPS ROIs, post-hoc paired $t$ tests showed that the activity between visible and invisible upright bodies did not differ ( $p$ IPS: $t(6)=1.166, p=.288$; mIPS: $t(6)=-0.040, p=.970$ ), but the activity between the two inverted conditions differed (pIPS: $t(6)=4.886, p=.003$, mIPS:

$t(6)=4.630, p=.004)$. See Figure 4 and Table 2.

In comparison to the ventral and dorsal ROIs, no significant main effect or interaction was observed for V1 ROIs (all p>.05).
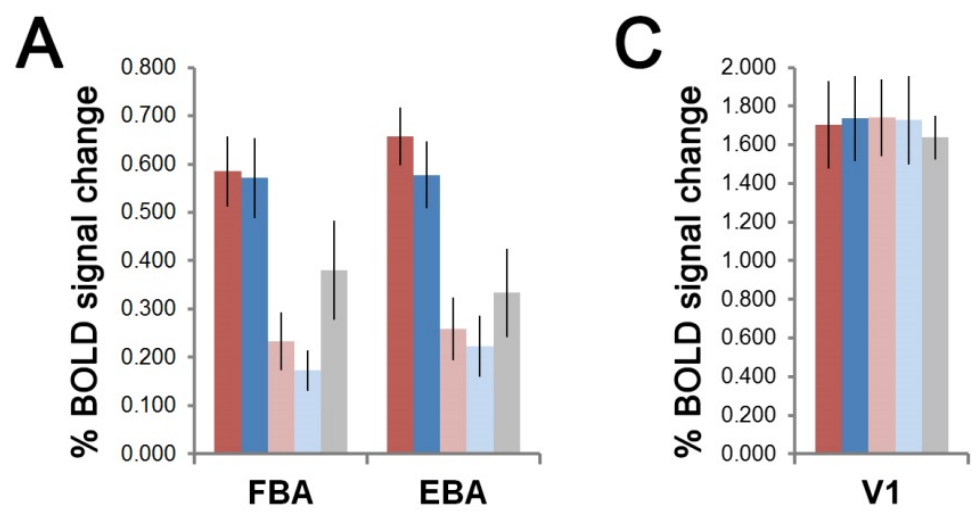

\section{Ventral ROls}

B

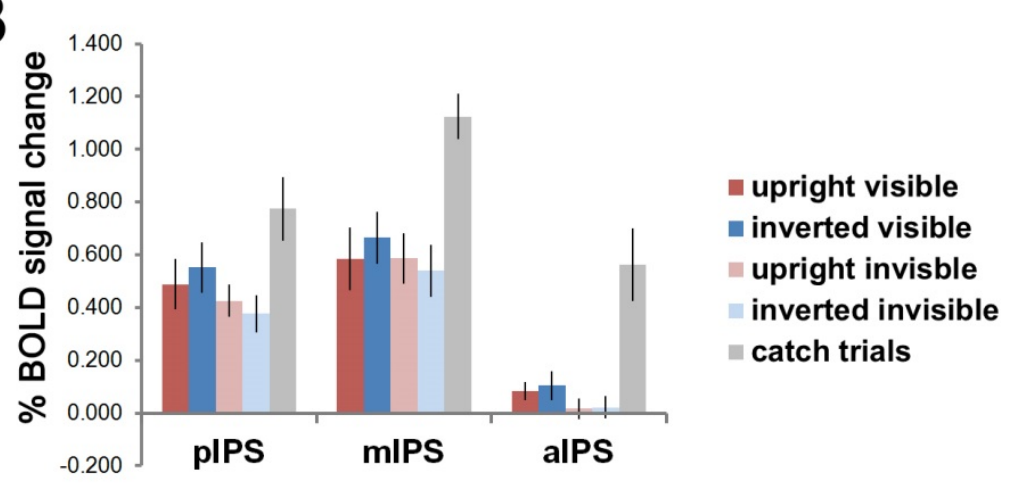

\section{Dorsal ROls}

Figure 4. The average percent signal change for the 4 main conditions and the catch trials (judging number of visible dots, followed by a button press) in ventral, dorsal, and V1 ROls. A. Ventral ROIs ( $n=7$ ). B. Dorsal ROIs ( $n=7$ for pIPS and mIPS, $n=6$ for alPS). C. V1 ROIs ( $n=7)$. Error bars denote standard errors of the mean. 


\section{Ventral and dorsal activity for body postures under CFS}

\section{ROI analysis in individual participants}

To rule out the possibility that the abovementioned ROI results were driven by a minority of participants, we performed within-participant repeated-measures ANOVAs in the bilateral ROls of the $\mathbf{7}$ individual participants. See Figure $\mathbf{5}$ and Table $\mathbf{3}$ for averaged responses per condition, the $p$ values for the statistical tests, and the directions of significant main effects.

The within-participant results in individual participants were consistent with the group results. ANOVA of stream (ventral/dorsal ROIs) $\times$ orientation $\times$ visibility showed significant interactions of stream $\times$ visibility in all 7 participants, while the main effect of visibility was present in 5 participants. The upright visible condition had higher activity than the upright invisible condition in 6 participants in the combined ventral ROI. In the combined dorsal ROI though, this comparison was not significant in any of the participants (all $p>.131$ ).

In individual ventral ROIs, consistent orientation effects was found in both the FBA and the EBA ROIs (6 out of 7 participants), which was the same case for pairwise comparisons of the upright visible versus invisible bodies.

In individual dorsal ROIs, two participants (S1 and S6) showed higher activity for visible trials, in pIPS and aIPS ROIs. In dorsal ROIs of other participants, the main effect of visibility was either non-significant, or showing the opposite effect to ventral ROls (higher activity for invisible trials than visible ones, in mIPS for 1 participant), or showing interactions of orientation and visibility (in pIPS for 1 participant, in mIPS for 2 participants). One participant further showed a main effect of higher activity for inverted bodies in both pIPS and mIPS, another showed the opposite effect in aIPS. Pairwise comparisons of upright visible and upright invisible conditions showed higher activity for the upright visible condition, in pIPS for 1 participant, and showed higher activity for the upright invisible condition in mIPS for 2 participants.

In the V1 ROI, one participant showed the main effect of visibility, showing higher activity for invisible bodies. For pairwise comparisons, another participant showed higher activity for upright visible bodies.

From these results, it appeared that the group-level effects were driven by the majority of the participants, and our results were consistent with the ones found by Fang \& He (2005). 


\section{Chapter 5}

Combined ROls

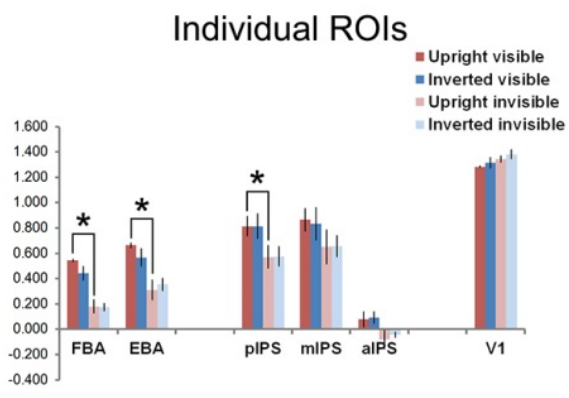

S2
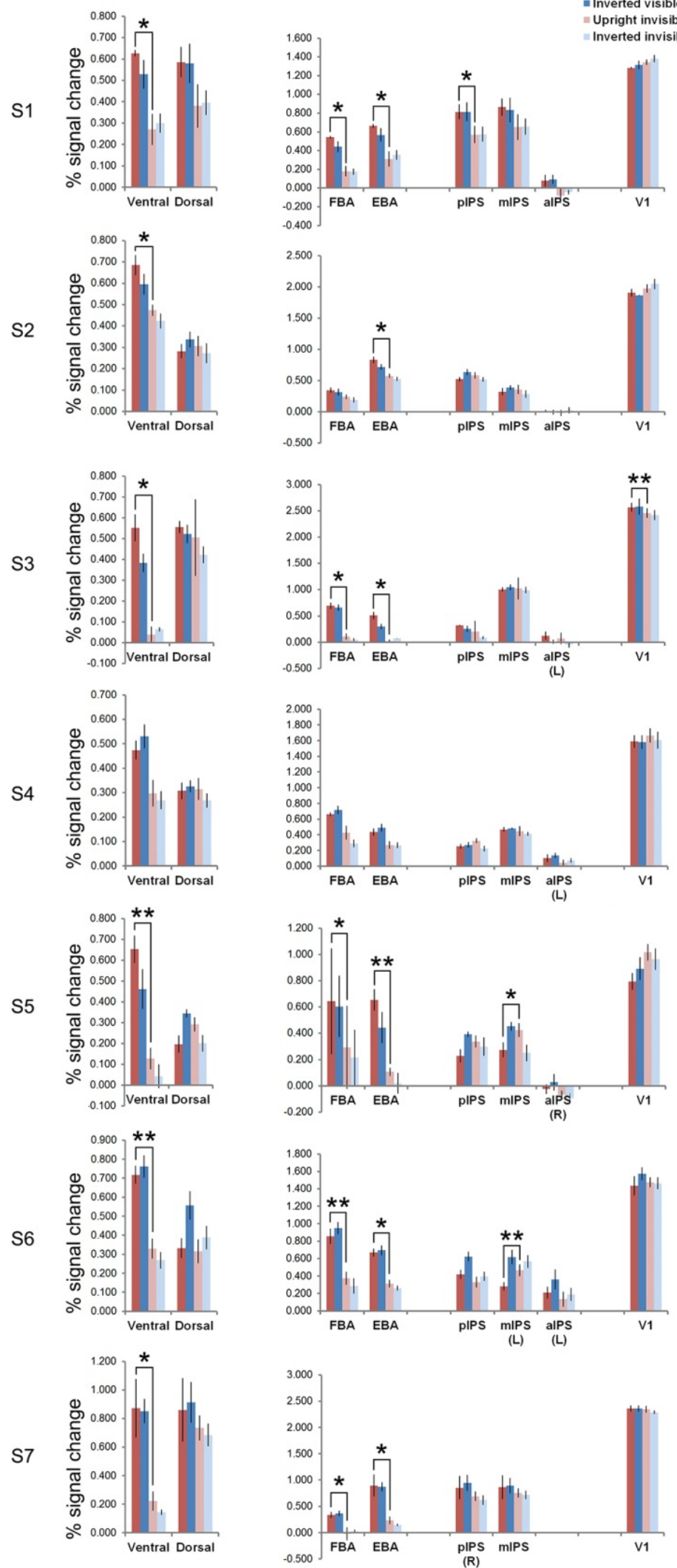

Figure 5. The average percent signal change for the 4 main conditions in the combined ventral and dorsal ROIs, and individual ROIs, at single-participant level. Unless marked, all ROIs included voxels from bilateral areas. Repeated-measures ANOVAs were performed in individual 
participants, with average percent signal changes (parameter estimates of the GLM) per run per condition as inputs. Error bars denote standard errors of the mean. For S1 and S3 the number of parameter estimates was 3 per condition, for the other 5 participants the number of parameter estimates was 4 per condition. Participants S2 and S5 are left handed. The significant pairwise comparisons between upright visible and upright invisible conditions were plotted. *: $p<.05$; **: $p<.01$. Note that most of the dorsal ROIs did not show significant activity differences between upright visible and upright invisible conditions. Furthermore, the significant effects in dorsal ROIs had opposite directions to the ones found in ventral ROIs. See Table $\mathbf{3}$ for the $p$ values of the ANOVAs and pairwise comparisons.

\section{Discussion}

Our results show that brain areas in the dorsal processing stream are substantially less associated with visual awareness, unlike the activity in ventral areas that is strongly linked to the visual awareness. Whole-brain ANOVA showed an interaction effect of stimulus orientation and visibility in regions including the IPS in the dorsal stream, and in subcortical structures. Also the ROI analysis showed a strong two-way interaction between stream and visibility (validated in within-participant analysis in all 7 participants), and a 3-way interaction of stream $\times$ orientation $\times$ visibility, while a main effect of visibility was also present. This overall difference between the two processing streams was caused by different response patterns in posterior and middle IPS ROIs more than the ventral and aIPS ROIs, with the former two areas showing an interaction between stimulus visibility and orientation. Specifically, activity in these two ROIs did not differ between the visible and invisible upright body stimuli. The FBA ROI also showed higher activity for upright bodies than inverted bodies, even when neither was consciously perceived.

The locations of our pIPS and mIPS ROIs correspond to the ROIs of V3A/V7 and IPS in the two previous fMRI CFS studies using tool stimuli (Fang \& He, 2005; Hesselmann \& Malach, 2011). Our finding that dorsal stream activity for upright body stimuli dissociates from visual awareness is consistent with the findings of Fang \& He (Fang \& He, 2005) using tools. The similarity between our results and theirs underscores that not only tools but also bodies trigger action representation, in which the IPS plays an important role (Culham et al., 2006). The other CFS studies did not find an interaction between stream and visibility, but found lower activity for invisible tools in both ventral and dorsal streams (Hesselmann et al., 2011; Hesselmann \& Malach, 2011; Ludwig et al., 2015). We also found this main effect of visibility in the dorsal areas, especially for the inverted bodies. However given the significant interaction between orientation and visibility, our evidence does not support an invariant processing across dorsal and ventral streams.

Previous reviews discussed explanations for the discrepancies between the available studies (Ludwig \& Hesselmann, 2015; Yang et al., 2014). One is that the presentation of visible trials was different (presented without dynamic noise in separate runs in Fang and $\mathrm{He} 2005$, but presented with dynamic noise in the same run in Hesselmann et al. 2011, Hesselmann and Malach 2011, Ludwig. et al 2015). Experiments of non-conscious tool perception have also 


\section{Chapter 5}

been criticized, with the reasoning that the results might be shape-specific and caused by the elongated shape only, rather than other tool-specific properties (Yang et al., 2014), although the elongated invisible tools indeed showed an enhanced decodability (Ludwig \& Hesselmann, 2015; Ludwig et al., 2015). However, our results show that these two reasons do not fully account for the previous discrepancies and underlying mechanisms. In our study visible and invisible trials were presented within the same run, always with the dynamic noise present. In addition, the body posture stimuli used in our study have elongated shapes in both upright and inverted forms, but body inversion led to a significant interaction with visibility in posterior and middle IPS, indicating that the underlying mechanisms for nonconscious tool and body perception are not likely to be shape-specific in purely lower-level visual-form aspects, but are more likely linked to higher-level processes associated with these two specific categories, especially their ability to trigger action-related processing.

The discrepancies between those studies may instead be caused by the averaging of activity in dorsal ROIs. Our ROI definition was more fine-grained, and gave the same weight to each dorsal ROI (same spherical ROI size across the 3 areas). We observed a change of response patterns at the group level along the IPS, where the interaction between orientation and visibility in posterior and middle IPS ROIs was not present in the anterior ROls, indicating a change of involvement and function across these areas, consistent to the functional heterogeneity found along the IPS in previous research (Freud, Plaut, \& Behrmann, 2016). Our change of responses was also consistent with the CFS study which specifically examined the decodabilities of faces and tools across two streams (Ludwig et al., 2016). In that study, the authors defined inferior and superior dorsal ROIs, roughly corresponding to a location posterior to our pIPS ROIs, and our mIPS ROI, respectively. They found that the decodability was modulated by the mask contrast in the superior dorsal ROls, but not so in the inferior dorsal ROIs. Taken together, the response patterns of the main conditions along the IPS are likely to be influenced by the size and location of the ROIs, and by the subsequent averaging of the BOLD responses. In view of the heterogeneity of response patterns along the IPS, further studies with higher functional resolution and fine-grained dorsal ROI definition will help to resolve the discrepancies.

Another possible reason for the discrepancies may be related to the active report of percept with button-press in the three previous studies (Hesselmann et al., 2011; Hesselmann \& Malach, 2011; Ludwig et al., 2015). Here we did not use active reports of visibility for each trial, because active reports under rivalry states induce significantly higher brain activity linked to introspection and action, mainly in frontal areas, but also in superior and inferior parietal areas (Frässle, Sommer, Jansen, Naber, \& Einhäuser, 2014). Since the medial and anterior IPS areas are known to be activated by hand actions such as touching, reaching and grasping (Culham et al., 2006), adding a button response per trial would introduce confounds in perceptual tasks aimed to compare ventral and dorsal activities. Indeed, we can observe the influence of a button press task in our data (Figure 3 and Figure 4), as we see that in the dorsal areas the activity for the catch trials was much higher than the main conditions. Further study explicitly comparing brain activity with/without active reports under CFS would shed more light on its actual influence to the dorsal activity. However, the no-report paradigm also has its limitations. Without explicit requirement of subjective reports like button presses, the participants may still form an implicit "report"; while in cases 
that a participant indeed consciously perceived a stimulus, the stimulus might either be forgotten, or below the participant's subjective report criteria, or not even reportable (Tsuchiya, Wilke, Frassle, \& Lamme, 2015). These between-participant variabilities could not all be assessed and accounted for by subjective reports, and would possibly have led to the discrepancies in the literature.

Lastly, the previous CFS studies differed in the length of inter-trial/block intervals, and in the data analysis methods. Fang \& He (2005) presented 20 s-blocks of faces and tools, interleaved with $20 \mathrm{~s}$-texture blocks as baseline), and only used the average signal of 8-20 s within each block. Together by presenting the invisible and visible conditions in different runs, there was no crosstalk of signals between conditions, and the BOLD signal dropped to baseline in the texture blocks (shown in their Figure 3 of objects/scrambled objects experiment. Their face/tool experiment was of a similar design). On the other hand, the 3 other studies used another design, with considerably shorter inter-trial intervals (1-6.5 s, 1.5-4.5 s, and 1-5 s) following the trial-by-trial awareness ratings with button presses, and used the GLM to estimate the BOLD signal changes. Under that specific design, the BOLD signal of the button press in the previous trial was likely to overlap with the upcoming trial despite the jitter of ITI, affecting all conditions. Our slow event-related design included sufficiently long inter-trial intervals, which allowed the BOLD response to return to baseline (in our case the average time for the BOLD response to return to baseline was 8-12 s after the end of dynamic noise presentation, see Figure 3). The next trial started 9-11 TRs after the previous trial onset, and started 1 additional TR later for catch trials with the presence of the response screen $(2 \mathrm{~s})$. Thus our design precluded any possible confounds related to effects carried over from preceding trials, and resulted in better estimation for responses of single conditions (Friston, Zarahn, Josephs, Henson, \& Dale, 1999).

The time course of the main conditions in Figure $\mathbf{3}$ seemingly showed double peaks, especially in the left pulvinar, with an early peak after trial onset, and a later peak after trial offset. Given the long ITI it is unlikely that this is due to contamination from previous trials. It might be related to the prolonged noise pattern displayed after the target stimulus offset (from 1.5 TR to 2.5 TR after stimulus onset to remove possible afterimages), during which the two eyes were still under a rivalrous situation, where the noise pattern was rivaling with the blank rectangle instead of the stimulus. This corresponded to the mask-only condition in two previous studies, where two related observations were made. Hesselmann et al. (2011) found that the activity of Mondrian mask-only trials were not significantly different from those of the invisible trials in the ROls they investigated, although showing a trend to significance in IPS. Ludwig et al. (2015) reconstructed the activity of invisible conditions by subtracting the mask-only activity from the mask-plus-stimulus activity, and found that parametrically modulating the mask contrasts did not show a corresponding difference in the activity in the ventral and dorsal ROls. Both observations suggest that the activity of invisible conditions under CFS was not modulated in an additive manner relating to the inputs of the two eyes. If the second peak in our data was also induced by the rivaling situation of blank rectangle and the noise pattern, it would question the validity of using the mask-only condition as a baseline. We could not disentangle the mask-only effect from the mask-plus-stimulus effect in our study, but future studies with higher temporal resolution may help understand better the mechanism of CFS. 


\section{Chapter 5}

Since we did not have subjective reports of visibility on a trial-to-trial basis, we used a different way of establishing suppression. We screened participants whose percept of stimuli was well suppressed by CFS, and then verified outside the scanner that their percepts closely follow our experimental manipulation of visibility on a trial-to-trial basis. The strict screening resulted in the relatively low number of participants in the current study, which may not well represent the whole population. To avoid creating differences of processing between the two hemispheres, we balanced the presentation of the noise pattern across the two eyes in both the screening and fMRI experiments. A recent study found that the CFS presentation 3 - 15 min into one eye would enhance its dominance in a subsequent presentation of binocular rivalry (Kim, Kim, \& Blake, 2017). Although we used short trials, as our screening experiments are relatively long (0.5-1 hour), this may contribute to understand why we did not find a large number of participants whose percept of the stimuli was fully suppressed under CFS. In the current fMRI experiment, there was the possibility that the stimuli occasionally broke the suppression for some participants. If this was the case, the activity for the invisible conditions in the ventral ROls could be affected. However, this cannot account for the sustained activity we observed in the dorsal ROIs for upright bodies. Thus our findings are robust in the participants we examined. Future studies may benefit from higher sample size, but may also benefit from experimental designs that are less demanding on the performance of the participants.

Activity in the posterior part of the IPS (pIPS and mIPS ROIs), apart from the possibility that it is linked to the action-perception-related aspect of bodies and tools, may also reflect a more general attentional mechanism triggered by the stimulus. The IPS is part of the dorsal attention network and is known to be activated in multiple tasks. It is involved in the direction of attention, eye movements, and detection of salient events (Corbetta \& Shulman, 2011), all of which could have played a role in our experiment. We presented the stimuli in the center of the visual fields, and instructed the participants to always fixate centrally on the fixation cross, thus the voluntary spatial attention of participants was always directed to the center of the rectangles. We could not rule out the possibility that there may be a difference of microsaccades between visible and invisible trials, as a previous CFS study found an increase of gaze directing to the locations of invisible stimuli than contralateral control locations (Rothkirch, Stein, Sekutowicz, \& Sterzer, 2012). Also, activity in the ventral pathway is known to be modulated by attention (Gilbert \& Li, 2013), and an attentional modulation of activity was found under CFS as early as V1 in spite of visibility (Watanabe et al., 2011). However, since the subjective percepts of the invisible trials were the same, the attentional mechanism alone could not explain the higher activity we found for invisible upright than inverted bodies in the FBA ROI. Instead, an interplay of dorsal and ventral mechanisms may be present, as recent research suggested for object perception (Freud et al., 2016). In our case, after the information from invisible body stimuli is relayed to both dorsal and ventral pathways, the ventral pathway representation may gain a categoryspecific processing advantage based on shape and orientation of the upright bodies, and the dorsal pathway representation may gain an advantage relating to action-observationexecution information in the upright bodies. These two in turn may drive the involuntary attention and affect the microsaccades. 
The human posterior IPS regions may be homologous to the lateral intraparietal area (LIP) in monkeys (Culham \& Kanwisher, 2001), whose activity is modulated by stimulus salience and behavioral relevance (Baluch \& Itti, 2011; Corbetta \& Shulman, 2002). In our study the interaction between orientation and visibility is shown in the posterior and middle IPS ROIs as well as in the dorsal attentional network clusters from the whole-brain ANOVA. The interaction found in posterior part of IPS may reflect a salience competition between the body stimuli and the dynamic noise pattern, caused by binocular disparity. If so, the salience and behavioral relevance of body stimuli may well result from the interplay between ventral and dorsal mechanisms. Given that under CFS salient and behaviorally-relevant stimuli were found to break through suppression faster (Yang et al., 2014), our findings suggest that the posterior part of IPS may act as an important transition stage in mediating stimuli entering into awareness by representing the salience of the stimuli. Our findings add to the link between visual perception and action, and are relevant for understanding the neural basis of perception of affective stimuli outside awareness. 


\section{Chapter 5}

\section{References}

Baluch, F., \& Itti, L. (2011). Mechanisms of top-down attention. Trends Neurosci, 34(4), 210-224. Brainard, D. H. (1997). The psychophysics toolbox. Spatial vision, 10, 433-436.

Carey, D. P., Harvey, M., \& Milner, A. D. (1996). Visuomotor sensitivity for shape and orientation in a patient with visual form agnosia. Neuropsychologia, 34(5), 329-337.

Corbetta, M., \& Shulman, G. L. (2002). Control of goal-directed and stimulus-driven attention in the brain. Nat Rev Neurosci, 3(3), 201-215. doi: 10.1038/nrn755

Corbetta, M., \& Shulman, G. L. (2011). Spatial neglect and attention networks. Annu Rev Neurosci, 34, 569-599. doi: 10.1146/annurev-neuro-061010-113731

Culham, J. C., Cavina-Pratesi, C., \& Singhal, A. (2006). The role of parietal cortex in visuomotor control: what have we learned from neuroimaging? Neuropsychologia, 44(13), 2668-2684. doi: 10.1016/j.neuropsychologia.2005.11.003

Culham, J. C., \& Kanwisher, N. (2001). Neuroimaging of cognitive functions in human parietal cortex. Curr Opin Neurobiol, 11(2), 157-163.

de Gelder, B., Tamietto, M., Van Boxtel, G., Goebel, R., Sahraie, A., Van den Stock, J., . . Pegna, A. (2008). Intact navigation skills after bilateral loss of striate cortex. Curr Biol, 18(24), R11281129. doi: 10.1016/j.cub.2008.11.002

de Gelder, B., \& Van den Stock, J. (2011). The Bodily Expressive Action Stimulus Test (BEAST). Construction and Validation of a Stimulus Basis for Measuring Perception of Whole Body Expression of Emotions. Front Psychol, 2, 181. doi: 10.3389/fpsyg.2011.00181

Fang, F., \& He, S. (2005). Cortical responses to invisible objects in the human dorsal and ventral pathways. Nat Neurosci, 8(10), 1380-1385. doi: 10.1038/nn1537

Frässle, S., Sommer, J., Jansen, A., Naber, M., \& Einhäuser, W. (2014). Binocular rivalry: frontal activity relates to introspection and action but not to perception. The Journal of neuroscience, 34(5), 1738-1747.

Freud, E., Plaut, D. C., \& Behrmann, M. (2016). 'What' Is Happening in the Dorsal Visual Pathway. Trends Cogn Sci, 20(10), 773-784. doi: 10.1016/j.tics.2016.08.003

Friston, K. J., Zarahn, E., Josephs, O., Henson, R., \& Dale, A. M. (1999). Stochastic designs in eventrelated fMRI. Neuroimage, 10(5), 607-619.

Gilaie-Dotan, S., Gelbard-Sagiv, H., \& Malach, R. (2010). Perceptual shape sensitivity to upright and inverted faces is reflected in neuronal adaptation. Neuroimage, 50(2), 383-395. doi: 10.1016/j.neuroimage.2009.12.077

Gilbert, C. D., \& Li, W. (2013). Top-down influences on visual processing. Nat Rev Neurosci, 14(5), 350363. doi: $10.1038 / \mathrm{nrn} 3476$

Hesselmann, G., Hebart, M., \& Malach, R. (2011). Differential BOLD activity associated with subjective and objective reports during "blindsight" in normal observers. J Neurosci, 31(36), 1293612944. doi: 10.1523/JNEUROSCI.1556-11.2011

Hesselmann, G., \& Malach, R. (2011). The link between fMRI-BOLD activation and perceptual awareness is "stream-invariant" in the human visual system. Cereb Cortex, 21(12), 2829-2837. doi: $10.1093 /$ cercor/bhr085

Jiang, Y., \& He, S. (2006). Cortical responses to invisible faces: dissociating subsystems for facialinformation processing. Curr Biol, 16(20), 2023-2029. doi: 10.1016/j.cub.2006.08.084

Johnson-Frey, S. H. (2004). The neural bases of complex tool use in humans. Trends Cogn Sci, 8(2), 7178.

Karnath, H. O., Himmelbach, M., \& Rorden, C. (2002). The subcortical anatomy of human spatial neglect: putamen, caudate nucleus and pulvinar. Brain, 125(2), 350-360. 


\section{Ventral and dorsal activity for body postures under CFS}

Kim, H. W., Kim, C. Y., \& Blake, R. (2017). Monocular Perceptual Deprivation from Interocular Suppression Temporarily Imbalances Ocular Dominance. Curr Biol, 27(6), 884-889. doi: 10.1016/j.cub.2017.01.063

Ludwig, K., \& Hesselmann, G. (2015). Weighing the evidence for a dorsal processing bias under continuous flash suppression. Conscious Cogn, 35, 251-259. doi:

10.1016/j.concog.2014.12.010

Ludwig, K., Kathmann, N., Sterzer, P., \& Hesselmann, G. (2015). Investigating category- and shapeselective neural processing in ventral and dorsal visual stream under interocular suppression. Hum Brain Mapp, 36(1), 137-149. doi: 10.1002/hbm.22618

Ludwig, K., Sterzer, P., Kathmann, N., Franz, V. H., \& Hesselmann, G. (2013). Learning to detect but not to grasp suppressed visual stimuli. Neuropsychologia, 51(13), 2930-2938. doi: 10.1016/j.neuropsychologia.2013.09.035

Ludwig, K., Sterzer, P., Kathmann, N., \& Hesselmann, G. (2016). Differential modulation of visual object processing in dorsal and ventral stream by stimulus visibility. Cortex, 83, 113-123. doi: 10.1016/j.cortex.2016.07.002

Lundqvist, D., Flykt, A., \& Öhman, A. (1998). The Karolinska directed emotional faces (KDEF). CD ROM from Department of Clinical Neuroscience, Psychology section, Karolinska Institutet, 91-630.

Lupyan, G., \& Ward, E. J. (2013). Language can boost otherwise unseen objects into visual awareness. Proc Natl Acad Sci U S A, 110(35), 14196-14201. doi: 10.1073/pnas.1303312110

Mastropasqua, T., Tse, P. U., \& Turatto, M. (2015). Learning of monocular information facilitates breakthrough to awareness during interocular suppression. Atten Percept Psychophys, 77(3), 790-803. doi: 10.3758/s13414-015-0839-z

McIntosh, R. D., McClements, K. I., Schindler, I., Cassidy, T. P., Birchall, D., \& Milner, A. D. (2004). Avoidance of obstacles in the absence of visual awareness. Proc Biol Sci, 271(1534), 15-20. doi: $10.1098 /$ rspb.2003.2545

Milner, A. D. (2012). Is visual processing in the dorsal stream accessible to consciousness? Proc Biol Sci, 279(1737), 2289-2298. doi: 10.1098/rspb.2011.2663

Milner, A. D., \& Goodale, M. A. (2006). The visual brain in action: Oxford University Press.

Peelen, M. V., \& Downing, P. E. (2007). The neural basis of visual body perception. Nat Rev Neurosci, 8(8), 636-648. doi: 10.1038/nrn2195

Pelli, D. G. (1997). The VideoToolbox software for visual psychophysics: Transforming numbers into movies. Spatial vision, 10(4), 437-442.

Pinsk, M. A., Arcaro, M., Weiner, K. S., Kalkus, J. F., Inati, S. J., Gross, C. G., \& Kastner, S. (2009). Neural representations of faces and body parts in macaque and human cortex: a comparative FMRI study. J Neurophysiol, 101(5), 2581-2600.

Reed, C. L., Stone, V. E., Bozova, S., \& Tanaka, J. (2003). The body-inversion effect. Psychol Sci, 14(4), 302-308.

Rizzolatti, G., \& Sinigaglia, C. (2010). The functional role of the parieto-frontal mirror circuit: interpretations and misinterpretations. Nature Reviews Neuroscience, 11(4), 264-274.

Rothkirch, M., Stein, T., Sekutowicz, M., \& Sterzer, P. (2012). A direct oculomotor correlate of unconscious visual processing. Curr Biol, 22(13), R514-515. doi: 10.1016/j.cub.2012.04.046

Schurger, A. (2009). A very inexpensive MRI-compatible method for dichoptic visual stimulation. J Neurosci Methods, 177(1), 199-202.

Stein, T., \& Peelen, M. V. (2015). Content-specific expectations enhance stimulus detectability by increasing perceptual sensitivity. J Exp Psychol Gen, 144(6), 1089-1104. doi: 10.1037/xge0000109

Stein, T., Sterzer, P., \& Peelen, M. V. (2012). Privileged detection of conspecifics: evidence from inversion effects during continuous flash suppression. Cognition, 125(1), 64-79. doi: 10.1016/j.cognition.2012.06.005 


\section{Chapter 5}

Stienen, B. M., \& de Gelder, B. (2011). Fear detection and visual awareness in perceiving bodily expressions. Emotion, 11(5), 1182-1189. doi: 10.1037/a0024032

Tsuchiya, N., \& Koch, C. (2005). Continuous flash suppression reduces negative afterimages. Nat Neurosci, 8(8), 1096-1101. doi: 10.1038/nn1500

Tsuchiya, N., Koch, C., Gilroy, L. A., \& Blake, R. (2006). Depth of interocular suppression associated with continuous flash suppression, flash suppression, and binocular rivalry. J Vis, 6(10), 10681078. doi: 10.1167/6.10.6

Tsuchiya, N., Wilke, M., Frassle, S., \& Lamme, V. A. (2015). No-Report Paradigms: Extracting the True Neural Correlates of Consciousness. Trends Cogn Sci, 19(12), 757-770. doi: 10.1016/j.tics.2015.10.002

Van den Stock, J., Tamietto, M., Zhan, M., Heinecke, A., Hervais-Adelman, A., Legrand, L. B., . . . de Gelder, B. (2014). Neural correlates of body and face perception following bilateral destruction of the primary visual cortices. Front Behav Neurosci, 8, 30. doi: 10.3389/fnbeh.2014.00030

Watanabe, M., Cheng, K., Murayama, Y., Ueno, K., Asamizuya, T., Tanaka, K., \& Logothetis, N. (2011). Attention but not awareness modulates the BOLD signal in the human V1 during binocular suppression. Science, 334(6057), 829-831. doi: 10.1126/science.1203161

Yang, E., Brascamp, J., Kang, M. S., \& Blake, R. (2014). On the use of continuous flash suppression for the study of visual processing outside of awareness. Front Psychol, 5, 724. doi: 10.3389/fpsyg.2014.00724

Zhan, M., Hortensius, R., \& de Gelder, B. (2015). The Body as a Tool for Anger Awareness-Differential Effects of Angry Facial and Bodily Expressions on Suppression from Awareness. PLoS One, 10(10), e0139768. 


\section{Tables}

Table 1. List of clusters shown by the whole-brain ANOVA.

visibility $\mathrm{x}$ orientation Interactions

\begin{tabular}{|c|c|c|c|c|c|c|c|c|c|c|c|c|}
\hline Region & Tal X & Y & z & stdev $x$ & stdev y & stdev z & size $\left(\mathrm{mm}^{3}\right)$ & peak $x$ & peak y & peak z & peak $F$ & peak $p$ \\
\hline R central sulcus & 41.88 & -20.88 & 54 & 1.54 & 1.69 & 1.12 & 78 & 43 & -21 & 54 & 41.895 & 0.0006 \\
\hline $\mathrm{R}$ central sulcus & 43.3 & -13.75 & 52.39 & 1.02 & 1.4 & 1.06 & 56 & 43 & -14 & 52 & 39.4 & 0.0008 \\
\hline $\mathrm{R}$ inferior precentral sulcus & 39.34 & -4.53 & 51.5 & 1.69 & 1.35 & 1.74 & 116 & 39 & -4 & 52 & 44.075 & 0.0006 \\
\hline $\mathrm{R}$ middle frontal sulcus, anterior & 32.79 & 28.8 & 34.09 & 2.22 & 1.53 & 2.12 & 178 & 31 & 29 & 35 & 88.547 & $8 \mathrm{E}-05$ \\
\hline R superior frontal sulcus & 29.47 & -2.43 & 53.41 & 1.27 & 1.34 & 1.14 & 79 & 30 & -2 & 54 & 65.446 & 0.0002 \\
\hline R superior frontal sulcus & 27.61 & 3.46 & 62.25 & 1.32 & 1.32 & 1.24 & 59 & 27 & 4 & 63 & 39.093 & 0.0008 \\
\hline R posterior IPS (connecting to the mIPS) & 18.2 & -64.4 & 38.09 & 1.66 & 1.1 & 1.35 & 82 & 17 & -64 & 39 & 49.916 & 0.0004 \\
\hline R superior frontal gyrus (SEF) & 15.95 & 0.19 & 64.86 & 0.96 & 0.79 & 2.15 & 64 & 16 & 0 & 66 & 43.332 & 0.0006 \\
\hline R caudate & 13.82 & 4.62 & 17.39 & 1.42 & 2.14 & 0.84 & 74 & 13 & 6 & 17 & 53.046 & 0.0003 \\
\hline $\mathrm{R}$ posterior cingulate sulcus & 4.15 & 7.9 & 45.11 & 1.25 & 1.64 & 1.86 & 88 & 3 & 7 & 47 & 43.572 & 0.0006 \\
\hline L precuneus, inferior & -3.76 & -69.67 & 36.48 & 1.09 & 0.96 & 1.51 & 54 & -3 & -69 & 36 & 69.414 & 0.0002 \\
\hline L precuneus, superior & -7.26 & -69.85 & 45.47 & 1.48 & 1.63 & 0.98 & 95 & -8 & -70 & 46 & 41.893 & 0.0006 \\
\hline L pulvinar & -9.71 & -17.89 & 13.16 & 1.47 & 0.98 & 0.98 & 56 & -10 & -18 & 13 & 33.34 & 0.0012 \\
\hline L superior parietal gyrus & -10.41 & -60.28 & 58.01 & 1.14 & 1.71 & 2.48 & 75 & -10 & -62 & 56 & 72.816 & 0.0001 \\
\hline L posterior IPS & -25.1 & -64.65 & 56.47 & 2.19 & 1.43 & 1.31 & 104 & -24 & -65 & 57 & 49.793 & 0.0004 \\
\hline L middle frontal gyrus & -26.35 & -9.48 & 58.7 & 1.02 & 1.27 & 1.26 & 54 & -27 & -10 & 59 & 33.024 & 0.0012 \\
\hline L middle frontal sulcus/superior frontal sulcus & -27.89 & 26.62 & 38.79 & 1.01 & 1.3 & 1.53 & 61 & -28 & 27 & 38 & 43.902 & 0.0006 \\
\hline L posterior insula & -33.76 & -25.47 & 22.4 & 1.04 & 0.97 & 1.26 & 58 & -34 & -26 & 23 & 56.325 & 0.0003 \\
\hline
\end{tabular}

main effect orientation

\begin{tabular}{|c|c|c|c|c|c|c|c|c|c|c|c|c|}
\hline Region & Tal $\mathrm{X}$ & Y & $\mathbf{z}$ & $\operatorname{stdev} x$ & stdev y & $\operatorname{stdev} z$ & $\operatorname{size}\left(\mathrm{mm}^{3}\right)$ & peak $\mathrm{x}$ & peak y & peak z & peak $\mathrm{F}$ & peak $p$ \\
\hline $\mathrm{R}$ middle frontal gyrus & 38.47 & 38.18 & 22.25 & 1.35 & 1.04 & 1.25 & 51 & 38 & 38 & 22 & 33.466 & 0.0012 \\
\hline R superior frontal gyrus, anterior & 18.33 & 55.51 & 26.98 & 0.94 & 1.39 & 0.96 & 55 & 18 & 56 & 27 & 36.501 & 0.0009 \\
\hline $\mathrm{R}$ superior frontal sulcus, anterior & 14.96 & 54.72 & 6.28 & 1.22 & 0.92 & 1.92 & 72 & 14 & 54 & 5 & 66.051 & 0.0002 \\
\hline L anterior cingulate sulcus & -15.02 & 21.98 & 31.78 & 1.16 & 1.1 & 1.12 & 59 & -15 & 22 & 32 & 89.787 & $8 \mathrm{E}-05$ \\
\hline L superior frontal sulcus/gyrus & -21.33 & 50.2 & 31.18 & 3.47 & 2.41 & 2.57 & 306 & -25 & 49 & 32 & 59.614 & 0.0002 \\
\hline L superior frontal sulcus/gyrus & -21.66 & 52.66 & 14.17 & 0.88 & 1.61 & 1.03 & 59 & -22 & 52 & 14 & 36.22 & 0.0009 \\
\hline $\begin{array}{l}\text { L inferior frontal sulcus \& inferior prec } \\
\text { connection point }\end{array}$ & -52.39 & 8.62 & 34.87 & 1.04 & 1.63 & 1.48 & 69 & -53 & 7 & 35 & 36.063 & 0.001 \\
\hline L lateral temporal gyrus (anterior to th & & & & & & & & & & & & \\
\hline in 6 participants, overlapping with 1 ) & -59.31 & -58.91 & 0.67 & 1.23 & 1.55 & 1.19 & 91 & -59 & -60 & 1 & 49.214 & 0.0004 \\
\hline
\end{tabular}

main effect visibility

\begin{tabular}{|c|c|c|c|c|c|c|c|c|c|c|c|c|}
\hline Region & Tal X & Y & $\mathbf{z}$ & $\operatorname{stdev} x$ & stdev y & $\operatorname{stdev} z$ & size $\left(\mathrm{mm}^{3}\right)$ & peak $x$ & peak y & peak z & peak $\mathrm{F}$ & peak $p$ \\
\hline $\mathrm{R}$ lateral occipital sulcus (EBA) & 49.1 & -70.36 & 16.38 & 2.73 & 2.85 & 3.49 & 400 & 49 & -69 & 18 & 51.073 & 0.0004 \\
\hline R inferior frontal gyrus, pars opercularis & 50.75 & 11.83 & 13.1 & 1 & 1.14 & 1.19 & 59 & 51 & 12 & 13 & 51.765 & 0.0004 \\
\hline R lateral occipital sulcus (EBA) & 47.7 & -77.04 & 12.61 & 1.94 & 1.7 & 1.8 & 148 & 46 & -78 & 13 & 57.856 & 0.0003 \\
\hline $\mathrm{R}$ inferior frontal gyrus, pars obitalis & 48.53 & 28.97 & -2.96 & 1.7 & 1.56 & 1.67 & 154 & 47 & 28 & -4 & 47.69 & 0.0005 \\
\hline R lateral occipital sulcus (EBA) & 47.55 & -61.26 & 12.18 & 1.29 & 0.88 & 1.74 & 76 & 48 & -61 & 12 & 43.297 & 0.0006 \\
\hline R inferior occipital gyrus (Lateral occipital complex) & 44.39 & -71.09 & -10.71 & 2.23 & 4.49 & 6.14 & 516 & 46 & -70 & -16 & 45.839 & 0.0005 \\
\hline
\end{tabular}




\section{Chapter 5}

\begin{tabular}{|c|c|c|c|c|c|c|c|c|c|c|c|c|}
\hline R fusiform gyrus (FBA) & 38.62 & -44.05 & -16.4 & 3.99 & 6.48 & 2.04 & 1050 & 38 & -44 & -18 & 123.67 & $3 E-05$ \\
\hline R IPS, anterior branch & 39.93 & -37.16 & 46.85 & 3.57 & 3.15 & 2.79 & 525 & 39 & -41 & 49 & 92.748 & 7E-05 \\
\hline $\mathrm{R}$ inferior frontal gyrus, pars obitalis & 37.37 & 40.1 & 10.15 & 1.46 & 2.13 & 1.44 & 157 & 37 & 39 & 9 & 61.047 & 0.0002 \\
\hline R collateral gyrus, anterior (anterior temporal lobe) & 36.74 & -10.13 & -28.58 & 2.14 & 1.15 & 0.98 & 89 & 35 & -9 & -29 & 65.553 & 0.0002 \\
\hline R middle frontal gyrus (FEF) & 30.73 & 1.75 & 63.45 & 1.09 & 1.18 & 0.94 & 56 & 31 & 2 & 64 & 70.458 & 0.0002 \\
\hline R superior frontal sulcus & 23.78 & 10.87 & 57.48 & 1.26 & 1.28 & 0.81 & 54 & 24 & 11 & 57 & 45.353 & 0.0005 \\
\hline $\mathrm{R}$ posterior cingulate sulcus & 10.69 & -25.79 & 42.11 & 1.07 & 1.39 & 1.17 & 62 & 11 & -26 & 42 & 43.09 & 0.0006 \\
\hline L fourth occipital gyrus & -23.27 & -88.1 & -8.6 & 2.29 & 1.28 & 0.93 & 97 & -25 & -87 & -9 & 127.47 & $3 \mathrm{E}-05$ \\
\hline L posterior collateral sulcus & -22.52 & -78.63 & -9.81 & 1.22 & 1.23 & 1.04 & 63 & -22 & -79 & -10 & 31.852 & 0.0013 \\
\hline \multicolumn{13}{|l|}{ LIPS, connection point of the middle branch } \\
\hline and the anterior branch & -30.19 & -51.12 & 40.18 & 1.13 & 1.23 & 1.25 & 73 & -30 & -51 & 40 & 63.971 & 0.0002 \\
\hline L collateral sulcus (anterior fusiform region) & -34.12 & -43.66 & -16.2 & 1.71 & 2.17 & 0.71 & 59 & -34 & -44 & -16 & 25.157 & 0.0024 \\
\hline L inferior occipital sulcus (EBA) & -45.19 & -68.53 & -6.68 & 4.17 & 2.18 & 1.62 & 337 & -40 & -71 & -8 & 70.051 & 0.0002 \\
\hline L lateral occipital sulcus (EBA) & -46.95 & -76.46 & -0.25 & 2.47 & 1.52 & 1.51 & 222 & -45 & -77 & -1 & 78.08 & 0.0001 \\
\hline
\end{tabular}

Contrast: upright invisible >inverted invisible

\begin{tabular}{|c|c|c|c|c|c|c|c|c|c|c|c|c|}
\hline Region & Tal X & $\mathbf{Y}$ & $\mathbf{z}$ & stdev $\mathrm{x}$ & stdev y & $\operatorname{stdev} z$ & size $\left(\mathrm{mm}^{3}\right)$ & peak $x$ & peak y & peak z & peak $\mathrm{F}$ & peak $\mathrm{p}$ \\
\hline $\mathrm{R}$ precentral sulcus & 50.41 & -2.81 & 43.21 & 2.07 & 2.84 & 1.97 & 172 & 48 & -2 & 44 & 7.1546 & 0.0004 \\
\hline $\mathrm{R}$ middle frontal gyrus & 45.89 & 20.4 & 45.28 & 1.65 & 4.77 & 1.7 & 194 & 45 & 25 & 45 & 6.5412 & 0.0006 \\
\hline $\mathrm{R}$ middle frontal gyrus & 42.49 & 19.05 & 37.05 & 2.6 & 1.73 & 3.25 & 238 & 41 & 18 & 34 & 6.1623 & 0.0008 \\
\hline R inferior occipital sulcus & 42.31 & -55.71 & -7.34 & 1.6 & 1.57 & 1.98 & 151 & 42 & -56 & -7 & 7.2599 & 0.0003 \\
\hline $\begin{array}{l}\text { R superior frontal sulcus/R superior frontal gyrus, } \\
\text { anterior }\end{array}$ & 24.58 & 52.78 & 24.29 & 6.43 & 6.45 & 4.8 & 1340 & 32 & 48 & 21 & 8.1961 & 0.0002 \\
\hline $\mathrm{R}$ middle frontal gyrus (FEF) & 32.99 & 9.7 & 48.79 & 1.64 & 4.32 & 1.69 & 243 & 34 & 5 & 48 & 5.8615 & 0.0011 \\
\hline $\mathrm{R}$ cerebellum & 20.5 & -72.99 & -24.53 & 3.77 & 1.76 & 2.78 & 240 & 19 & -72 & -27 & 7.4966 & 0.0003 \\
\hline R caudate & 14.79 & 3.99 & 17.44 & 1.34 & 2.68 & 1.43 & 151 & 15 & 1 & 16 & 9.4184 & $8 \mathrm{E}-05$ \\
\hline $\mathrm{R}$ medial frontal gyrus & 6.86 & 15.9 & 56.65 & 1.91 & 3.48 & 1.85 & 161 & 5 & 19 & 55 & 5.0247 & 0.0024 \\
\hline L superior frontal sulcus (FEF) & -22.89 & -5.04 & 53.37 & 1.61 & 3.29 & 5.05 & 377 & -24 & -4 & 55 & 6.5383 & 0.0006 \\
\hline $\begin{array}{l}\text { L inferior frontal sulcus \& inferior precentral sulcus, } \\
\text { connection point }\end{array}$ & -48.15 & 13.04 & 36.09 & 3.88 & 2.83 & 3.84 & 424 & -48 & 13 & 37 & 7.7126 & 0.0002 \\
\hline
\end{tabular}


Table 2. Results of ANOVAs in combined ventral and dorsal ROIs, and in ROIs of individual areas. Significant results are indicated with*.

\begin{tabular}{|c|c|c|c|c|c|}
\hline ROls & ANOVA & & $\mathbf{F}$ & $\mathbf{p}$ & $\eta_{p}^{2}$ \\
\hline \multicolumn{6}{|l|}{ combined ventral ROI, } \\
\hline \multirow[t]{10}{*}{ combined dorsal ROI } & stream $\mathrm{x}$ orientation $\mathrm{x}$ visibility & stream & 0.013 & 0.913 & 0.002 \\
\hline & $\mathrm{n}=7$ & orientation & 2.006 & 0.206 & 0.251 \\
\hline & & visibility & 33.370 & $* 0.001$ & 0.848 \\
\hline & & stream $\mathrm{x}$ orientation & 5.296 & 0.061 & 0.469 \\
\hline & & stream $\mathrm{x}$ visibility & 30.821 & $* 0.001$ & 0.837 \\
\hline & & orientation $\mathrm{x}$ visibility & 2.231 & 0.186 & 0.271 \\
\hline & & stream $\mathrm{x}$ orientation $\mathrm{x}$ visibility & 7.307 & 0.035 & 0.549 \\
\hline & stream x visibility (upright conditions only) & stream & 0.098 & 0.765 & 0.016 \\
\hline & $\mathrm{n}=7$ & visibility & 24.987 & $* 0.002$ & 0.806 \\
\hline & & stream $\mathrm{x}$ visibility & 34.612 & $* 0.001$ & 0.852 \\
\hline \multirow[t]{3}{*}{ combined ventral ROI } & orientation $\mathrm{x}$ visibility & orientation & 16.297 & $* 0.007$ & 0.731 \\
\hline & $\mathrm{n}=7$ & visibility & 38.063 & $* 0.001$ & 0.864 \\
\hline & & orientation $\mathrm{x}$ visibility & 0.425 & 0.538 & 0.066 \\
\hline \multirow[t]{3}{*}{ combined dorsal ROI } & orientation $\mathrm{x}$ visibility & orientation & 0.122 & 0.738 & 0.02 \\
\hline & $n=7$ & visibility & 9.172 & $* 0.023$ & 0.605 \\
\hline & & orientation $\mathrm{x}$ visibility & 13.624 & $* 0.01$ & 0.694 \\
\hline \multirow[t]{7}{*}{ dorsal ROIs: pIPS, mIPS, aIPS } & area $\mathrm{x}$ orientation $\mathrm{x}$ visibility & area & 9.962 & $* 0.004$ & 0.666 \\
\hline & $n=6$ & orientation & 0.192 & 0.679 & 0.037 \\
\hline & & visibility & 6.149 & 0.056 & 0.552 \\
\hline & & area $\mathrm{x}$ orientation & 0.131 & 0.879 & 0.026 \\
\hline & & area $\mathrm{x}$ visibility & 0.847 & 0.457 & 0.145 \\
\hline & & orientation $\mathrm{x}$ visibility & 10.853 & $* 0.022$ & 0.685 \\
\hline & & area $\mathrm{x}$ orientation $\mathrm{x}$ visibility & 9.449 & $* 0.005$ & 0.654 \\
\hline ROIs of Individual areas & ANOVA & & $\mathbf{F}$ & $\mathbf{p}$ & $\eta_{p}^{2}$ \\
\hline \multirow[t]{3}{*}{ V1 } & orientation $\mathrm{x}$ visibility & orientation & 0.628 & 0.458 & 0.095 \\
\hline & $n=7$ & visibility & 0.174 & 0.691 & 0.028 \\
\hline & & orientation $\mathrm{x}$ visibility & 2.882 & 0.14 & 0.324 \\
\hline \multirow[t]{3}{*}{ FBA } & orientation $\mathrm{x}$ visibility & orientation & 9.950 & $* 0.02$ & 0.624 \\
\hline & $n=7$ & visibility & 37.446 & $* 0.001$ & 0.862 \\
\hline & & orientation $\mathrm{x}$ visibility & 1.780 & 0.231 & 0.229 \\
\hline \multirow[t]{3}{*}{ EBA } & orientation $\mathrm{x}$ visibility & orientation & 13.230 & $* 0.011$ & 0.688 \\
\hline & $\mathrm{n}=7$ & visibility & 35.008 & $* 0.001$ & 0.854 \\
\hline & & orientation $\mathrm{x}$ visibility & 1.223 & 0.311 & 0.169 \\
\hline
\end{tabular}




\section{Chapter 5}

\begin{tabular}{|c|c|c|c|c|c|}
\hline \multirow[t]{3}{*}{ pIPS } & orientation $\mathrm{x}$ visibility & orientation & 0.056 & 0.821 & 0.009 \\
\hline & $n=7$ & visibility & 7.477 & $* 0.034$ & 0.555 \\
\hline & & orientation $\mathrm{x}$ visibility & 19.060 & $* 0.005$ & 0.761 \\
\hline \multirow[t]{3}{*}{ mIPS } & orientation $\mathrm{x}$ visibility & orientation & 0.201 & 0.669 & 0.032 \\
\hline & $n=7$ & visibility & 2.711 & 0.151 & 0.311 \\
\hline & & orientation $\mathrm{x}$ visibility & 10.111 & $* 0.019$ & 0.628 \\
\hline \multirow[t]{3}{*}{ alPS } & orientation $\mathrm{x}$ visibility & orientation & 0.202 & 0.672 & 0.039 \\
\hline & $n=6$ & visibility & 10.812 & $* 0.022$ & 0.684 \\
\hline & & orientation $\mathrm{x}$ visibility & 0.689 & 0.444 & 0.121 \\
\hline
\end{tabular}


Table 3. $P$ values of the ANOVAs and pairwise comparisons for within-participant analysis in ROIs of individual participants. The inputs were parameter estimates (percent signal changes) of GLM per run per condition. Significant results are indicated with *.

\begin{tabular}{|c|c|c|c|c|c|c|c|c|c|}
\hline Participant & & S1 & S2 & S3 & S4 & S5 & S6 & S7 & $\begin{array}{l}\text { Direction of effect (number of } \\
\text { participants showing an effect) }\end{array}$ \\
\hline Number of runs & & 3 & 4 & 3 & 4 & 4 & 4 & 4 & \\
\hline \multirow{7}{*}{$\begin{array}{l}\text { ventral/dorsal ROIs } \mathrm{x} \\
\text { orientation x visibility }\end{array}$} & & & & & & & & & ventral>dorsal (3) \\
\hline & stream & 0.190 & $0.002^{*}$ & $0.066^{*}$ & $0.027^{*}$ & 0.346 & $0.024^{*}$ & $0.011^{*}$ & dorsal>ventral (1) \\
\hline & orientation & 0.742 & 0.515 & 0.257 & 0.990 & 0.429 & 0.084 & 0.746 & \\
\hline & $\begin{array}{l}\text { visibility } \\
\text { stream } \mathrm{x}\end{array}$ & $0.049 *$ & 0.089 & 0.100 & $0.011^{*}$ & $0.005^{*}$ & $0.00003^{*}$ & $0.045^{*}$ & visible> invisible (5) \\
\hline & orientation & 0.541 & $0.010^{*}$ & 0.878 & 0.581 & $0.010^{*}$ & $0.010^{*}$ & 0.307 & (3) \\
\hline & $\begin{array}{l}\text { stream } \mathrm{x} \text { visibility } \\
\text { stream } \mathrm{x} \\
\text { orientation } \mathrm{x}\end{array}$ & $0.022 *$ & $0.002^{*}$ & $0.018^{*}$ & $0.002^{*}$ & $0.002^{*}$ & $0.0003^{*}$ & $0.001 *$ & (7) \\
\hline & visibility & 0.337 & 0.050 & 0.300 & 0.632 & $0.008^{*}$ & 0.374 & 0.591 & (1)(trend 1) \\
\hline \multicolumn{10}{|l|}{ Individual ROIs } \\
\hline \multirow[t]{3}{*}{ FBA } & orientation & 0.211 & 0.459 & 0.141 & 0.304 & 0.730 & 0.970 & 0.757 & \\
\hline & $\begin{array}{l}\text { visibility } \\
\text { orientation } \mathrm{x}\end{array}$ & $0.024 *$ & 0.146 & $0.018^{*}$ & $0.0004 *$ & $0.010^{*}$ & $0.00005^{*}$ & $0.015^{*}$ & visible>invisible (6) \\
\hline & visibility & 0.365 & 0.843 & 0.854 & 0.217 & 0.719 & 0.261 & 0.886 & \\
\hline \multirow[t]{3}{*}{ EBA } & orientation & 0.114 & 0.141 & 0.060 & 0.383 & 0.107 & 0.755 & 0.542 & \\
\hline & $\begin{array}{l}\text { visibility } \\
\text { orientation } \mathrm{x}\end{array}$ & 0.053 & $0.012^{*}$ & $0.034^{*}$ & $0.005^{*}$ & $0.004^{*}$ & $0.00008^{*}$ & $0.009 *$ & visible>invisible (6) (trend 1 ) \\
\hline & visibility & 0.323 & 0.544 & $0.035^{*}$ & 0.527 & 0.162 & 0.541 & 0.729 & (1) \\
\hline \multirow[t]{3}{*}{ pIPS } & orientation & 0.974 & 0.714 & 0.491 & 0.260 & 0.466 & $0.004^{*}$ & 0.760 & inverted>upright (1) \\
\hline & $\begin{array}{l}\text { visibility } \\
\text { orientation } x\end{array}$ & $0.047^{*}$ & 0.618 & 0.390 & 0.525 & 0.857 & $0.005^{*}$ & 0.193 & visible>invisible (2) \\
\hline & visibility & 0.975 & $0.032 *$ & 0.783 & 0.099 & 0.129 & 0.169 & 0.267 & (1) \\
\hline \multirow[t]{3}{*}{ mIPS } & orientation & 0.898 & 0.952 & 0.964 & 0.803 & 0.950 & $0.003 *$ & 0.972 & inverted>upright (1) \\
\hline & $\begin{array}{l}\text { visibility } \\
\text { orientation } \mathrm{x}\end{array}$ & 0.147 & 0.444 & 0.870 & 0.203 & 0.692 & $0.027^{*}$ & 0.425 & invisible>visible (1) \\
\hline & visibility & 0.894 & 0.089 & 0.849 & 0.654 & $0.011^{*}$ & $0.025^{*}$ & 0.685 & (2) \\
\hline \multirow[t]{3}{*}{ alPS } & orientation & 0.551 & 0.532 & $0.028^{*}$ & 0.385 & 0.804 & 0.131 & & upright>inverted (1) \\
\hline & $\begin{array}{l}\text { visibility } \\
\text { orientation } \mathrm{x}\end{array}$ & $0.029 *$ & 0.968 & 0.391 & 0.201 & 0.169 & $0.038^{*}$ & & visible>invisible (2) \\
\hline & visibility & 0.916 & 0.453 & 0.987 & 0.970 & 0.468 & 0.583 & & \\
\hline \multirow[t]{3}{*}{ V1 } & orientation & 0.315 & 0.350 & 0.887 & 0.740 & 0.712 & 0.267 & 0.707 & \\
\hline & $\begin{array}{l}\text { visibility } \\
\text { orientation } \mathrm{x}\end{array}$ & 0.075 & 0.106 & 0.101 & 0.252 & $0.014^{*}$ & 0.556 & 0.091 & invisible>visible (1) \\
\hline & visibility & 0.866 & 0.351 & 0.674 & 0.130 & 0.374 & 0.285 & 0.702 & \\
\hline \multicolumn{10}{|l|}{ Pairwise comparison of } \\
\hline \multirow[t]{2}{*}{ upright invisible } & ventral & $0.031 *$ & $0.020^{*}$ & $0.033^{*}$ & 0.083 & $0.005^{*}$ & $0.008 *$ & $0.033^{*}$ & (6) \\
\hline & dorsal & 0.131 & 0.360 & 0.830 & 0.894 & 0.068 & 0.739 & 0.581 & \\
\hline
\end{tabular}




\section{Chapter 5}

\begin{tabular}{|c|c|c|c|c|c|c|c|c|}
\hline FBA & $0.012^{*}$ & 0.105 & $0.034^{*}$ & 0.061 & 0.035* & $0.004^{*}$ & $0.036^{*}$ & $\begin{array}{l}\text { upright visible> upright invisible } \\
\text { (5) } \\
\text { upright visible>upright invisible }\end{array}$ \\
\hline EBA & $0.041^{*}$ & $0.014^{*}$ & $0.033^{*}$ & 0.095 & $0.007^{*}$ & $0.014^{*}$ & $0.036 *$ & $\begin{array}{l}\text { (6) } \\
\text { upright visible>upright invisible }\end{array}$ \\
\hline pIPS & $0.016^{*}$ & 0.115 & 0.625 & 0.176 & 0.189 & 0.171 & 0.458 & $\begin{array}{l}\text { (1) } \\
\text { upright invisible>upright visible }\end{array}$ \\
\hline mIPS & 0.168 & 0.119 & 0.955 & 0.760 & $0.043^{*}$ & $0.003^{*}$ & 0.646 & (2) \\
\hline alPS & 0.309 & 0.527 & 0.765 & 0.417 & 0.512 & 0.470 & & \\
\hline V1 & 0.189 & 0.416 & $0.004^{*}$ & 0.183 & 0.103 & 0.751 & 0.842 & $\begin{array}{l}\text { upright visible> upright invisible } \\
\text { (1) }\end{array}$ \\
\hline
\end{tabular}




\section{Chapter 6}

\section{Action categories are represented in a large distributed network in the brain - evidence from 7T fMRI}

Corresponding manuscript:

Zhan, M., Goebel, R., Vaessen, M., de Gelder, B. (in preparation). Action categories are represented in a large distributed network in the brain - evidence from 7T fMRI 


\section{Chapter 7}

\section{The influence of a short domestic-violence VR scenario on emotional face and body perception}

Corresponding manuscript:

Zhan, M., Seinfeld, S., Poyo Solanas, M., Vaessen, M., Slater, M., Sanchez-Vives, M. V., de Gelder, B. (in preparation). The influence of a short domestic-violence VR scenario on emotional face and body perception. 
Chapter 8

General discussion 


\section{Chapter 8}

In this thesis, we examined the perception of bodily postures, which were instrumental actions or were expressing emotions, under non-conscious and conscious viewing conditions.

For processing bodies, we found that they involve different ventral and dorsal brain areas comparing to processing faces. The same emotions (fear and anger) conveyed by bodily or facial expressions were also processed differently under the non-conscious viewing condition of CFS, showing the opposite pattern of suppression times. Different to faces, the passive observing of emotional bodies did not elicit amygdala activity as much as faces.

For processing emotions, we found that bodily emotions (fear and anger) showed different suppression times under CFS, and these two emotions interacted differently with the visible emotions when simultaneously presented beside the invisible ones. When we examined the conscious perception of instrumental and emotional bodily actions, we did not find multivariate evidence in the brain specific for emotional actions versus non-emotional ones, in areas suggested by univariate results (PMv and IFG); nor multivariate evidence for discriminating positive and negative valences, in vmPFC.

For non-conscious visual processing of bodies under CFS, we found that the dorsal activity was much less associated with the visual percept, and showed an interaction of body orientation and visibility. Thus we added one piece of evidence to the inconsistent literature, and discussed possibilities resulting in such inconsistency. Behaviorally, we found that our CFS result for the redundant target effect was more similar to hemianopia patients without blindsight, rather than blindsight and neglect patients. This indicates that the "non-conscious" processing is multi-faceted and likely involves multiple different mechanisms in different brain areas, rather than a single processing. For the temporal frequencies of the CFS itself, we found that the suppression effect was stronger at 4, 6, $8 \mathrm{~Hz}$, comparing to the $10 \mathrm{~Hz}$ frequency usually used in most of the CFS studies.

We discuss some of our findings and their implications in detail below.

\section{Body and emotion perception in the brain, and methodological considerations}

In Chapter 6, we found that conscious perception of static whole-body actions involved a network that showed univariate activation when comparing against fixation, including the EBA, FBA and pSTS showing a certain level of body specificity, the IPS, preSMA, PMd and $\mathrm{PMv}$ that related to action processing, and more higher-order clusters in the frontal and inferior frontal areas. These activation sites were consistent with previous literature related to body and action processing, and we further found that some of these areas (e.g. those showing body specificity) were implicated in processing action categories. However, we found that the information of action categories was also present in the right superior frontal sulcus, the medial brain, subcortical, and cerebellar areas, some of which did not show consistent univariate activation for any of the 10 action categories. This indicates that the processing of bodily actions involves a much larger distributed network than we previously thought. The results demand considerations about methodologies we used to study bodily 


\section{General discussion}

action and emotion perception, which in turn demand deeper theoretical considerations of these processes.

For the research of visual processing, an emphasis on the "specificity" of processing could be seen in previous studies. In studying semantic object categories such as faces, bodies, visual words, tools, researchers have been continuously trying to find areas that specifically process these object categories. Indeed, with $\mathrm{fMRI}$ studies in the ventral-lateral stream, areas have been found that showed specificity for faces (Kanwisher, McDermott, \& Chun, 1997), bodies (Downing, Jiang, Shuman, \& Kanwisher, 2001) and words (Cohen et al., 2000). For the tools, its category specificity in the dorsal stream is still under debate (Fang \& He, 2005; Hesselmann, Hebart, \& Malach, 2011; Hesselmann \& Malach, 2011; Ludwig, Kathmann, Sterzer, \& Hesselmann, 2015), see also Chapter 5.

The urge to find "specificity" in the current fMRI research could be traced back to the tradition and methodology of PET and FMRI studies. If contrasting the BOLD activation versus a baseline, which differs very much in visual or other properties to the conditions of research interest, a large swath of areas across the brain would be seen activated. To understand the processing that relates to a specific condition, another condition controlling the properties not of interest would be necessary to make the contrast map. With this localization approach, a name or concept would then be assigned to the clusters produced in such maps, be it one area (e.g. the fusiform face area, FFA), or multiple areas (the action observation network, also known as the mirror neuron network). Due to the limitation of coverage in earlier fMRI studies, to further understand the processing in these clusters, researchers focused on small parts of the brain or single brain areas/clusters with higher fMRI resolution (for an example see Baker et al., 2007). Up to now, multiple localization attempts have been made to map single categories or attributes onto the human brain, both in visual and other modalities (e.g. pitch in the auditory modality), and higher-order functions such as emotion and social interactions (Saxe \& Kanwisher, 2003). With the finding of the FFA, the methodology of functional localizers is also being widely used in $\mathrm{FMRI}$ research. With separate functional data, researchers first define ROls by proper contrasts that supposedly reveal the clusters of target specificity, which is the interest of the research, and then analyze the data of the main experiment in these ROls.

However, there are limitations for this contrasting method, both for $\mathrm{fMRI}$ studies and in general for all kinds of studies that compared only a few conditions. One crucial question is: which control/contrast condition is a proper condition? For visual processing, researchers have been mainly using object images of different categories, using contrasts such as faces > objects. However, a study found that when defining the FFA, the location and extent of the resulting cluster differed between the contrast faces>houses and the contrast faces $>$ scrambled stimuli (Berman et al., 2010). For emotions, researchers usually utilize the same stimulus category that varied in emotional information, using contrasts such as emotional > neutral stimuli. We similarly used this manipulation in our behavioral and fMRI studies. To further control the action aspect of bodily actions, we did not use completely static neutral bodies (standing still) in our studies, as has been the practice in facial emotion research, instead we compared emotional bodily actions to one or several neutral bodily actions (Chapters $\mathbf{2}$ and 3: calling on the phone, Chapter 6: 5 neutral action categories). In fact, we 


\section{Chapter 8}

found in the study of Chapter 6 that the multivariate representation for the standing still neutral bodies was significantly different than the other 9 bodily action categories in EBA. The univariate activation maps differ between completely standing still > baseline and phone $>$ baseline (see Figure 1), although directly contrasting phone and standing still did not show surface clusters at $p=.005$ (data not shown). Conjunction analysis of the 5 neutral actions showed consistent activation only in the ventral-lateral areas. The difference of activation in the dorsal and frontal areas indicates that, to use only one of the few neutral conditions as the control condition, the resulting activation patterns of emotional > neutral stimuli are likely to be different in dorsal and frontal areas.
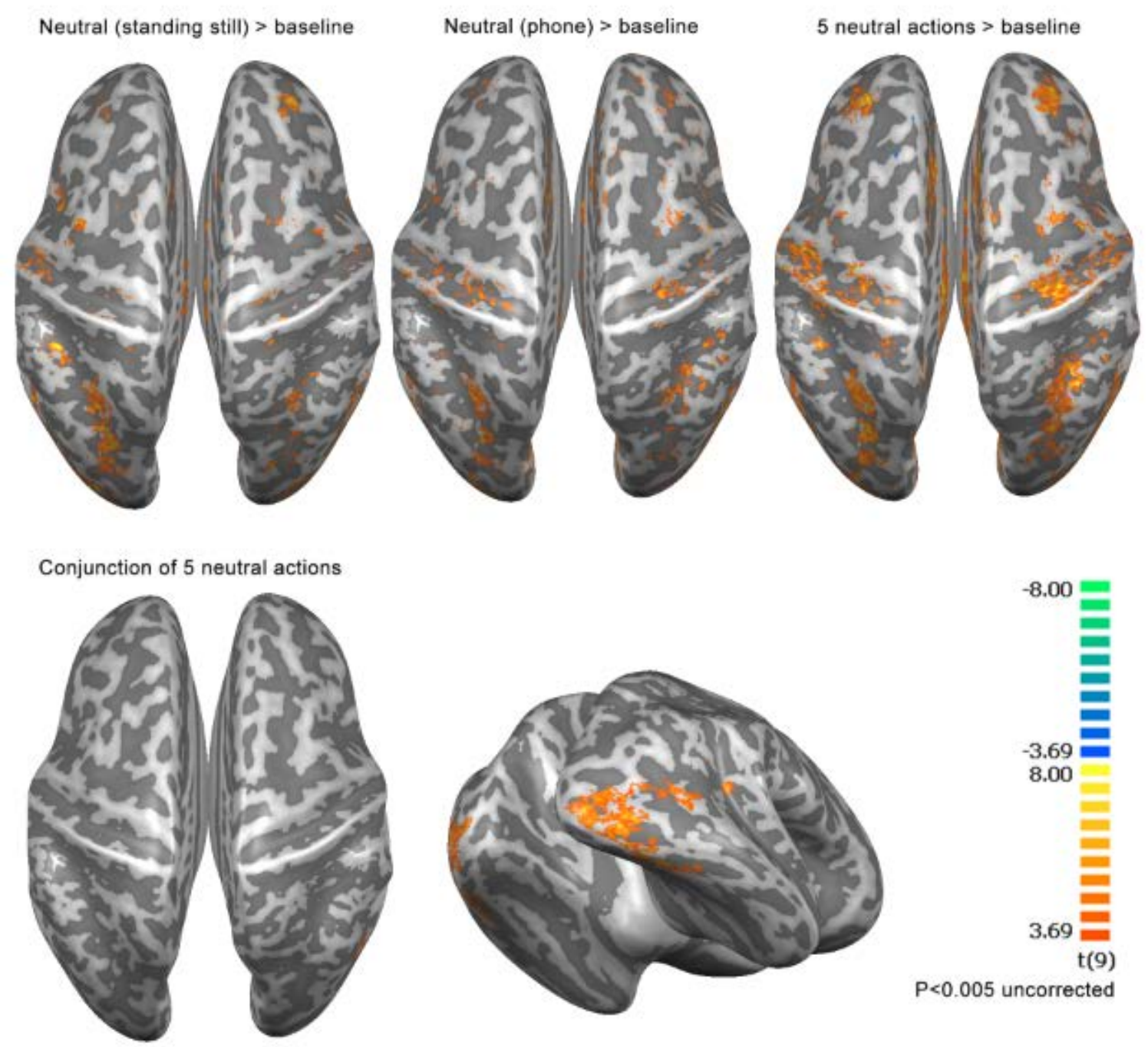

Figure 1. Univariate activation of neutral bodily actions and standing still bodies versus baseline (fixation), from the 10 participants in the 7T study (Chapter 6 ). The 5 neutral actions included combing hair, drinking water, opening door, calling on the phone, and putting on trousers.

When only limiting the control of the contrast condition in one or two attributes and using few conditions, this would limit the generalizability of multivariate results as well. In emotional research, because identity processing and visual form processing are important aspect of face and body processing, studies of facial and bodily emotions usually use stimuli of multiple identities to control for the stimulus variability in these two aspects. However, 


\section{General discussion}

other lower-level features also co-vary with the emotion itself, such as the eye-white size of the faces, and the action aspects of bodies, which may or may not be exhaustively controllable. When applying MVPA using the decoding approach, the decoding accuracy itself does not show the researcher which attribute was used/most informative in discriminating between conditions, or even obscures the direction of the decodability, e.g. when investigating which one of $A$ and $B$ categories was better processed in a region, either $A$ better processed than $B$, or $B$ better processed than $A$, would both give rise to an abovechance performance in individual participants (Allefeld, Gorgen, \& Haynes, 2016; Todd, Nystrom, \& Cohen, 2013). In such cases, when other attributes/features co-vary with emotion, above-chance decoding performances do not provide us information of the emotion per se. Previous MVPA studies found above-chance decoding performance for facial emotions in FFA and pSTS (Wegrzyn et al., 2015; Zhang et al., 2016), but these findings were not able to separate the emotion from lower-level visual covariations in the stimuli.

If the stimuli set is small in a study, there is also the danger that the results obtained by this specific stimuli set are not generalizable to other new stimuli sets. This is especially problematic for emotional studies with only a few stimuli exemplars per emotion category. For example, the amygdala thought to be implicated in emotion perception showed habituation effects during an emotional face matching task, and the habituation effect of amygdala was a more reliable index than its mean activation (Plichta et al., 2014). Researchers are starting to advocate treating stimuli as random variables in univariate analysis (Westfall, Nichols, \& Yarkoni, 2016). This practice is similar to the mixed effects analysis for behavioral data, which we adopted in chapter $\mathbf{2}$ and $\mathbf{4}$. For fMRI analysis, this approach is not yet readily implemented in the major analysis softwares. The use of naturalistic stimuli and task is also regarded important in recent years, and some studies found results inconsistent with those under artificial settings. For example, a study recorded the activity of amygdala neurons while the rat was foraging in a semi-naturalistic environment, but confronted by a robot predator. The study identified two cell types in the basolateral amygdala, and found that the firing rates of the two cell types was different during a waiting period and a foraging period, and only the activity of type- 1 cell during the waiting period was consistent with the "amygdala signals fear" hypothesis, which was obtained in artificial fear-conditioning settings (Amir, Lee, Headley, Herzallah, \& Pare, 2015; Paré \& Quirk, 2017). In most of our studies, we used large amount of stimuli exemplars that have naturalistic body appearances (with daily clothing. We only removed the facial information, but not the hair or the head). We also used virtual reality (VR) to create a naturalistic emotional scenario in Chapter 7, although we did not capture the online perception of VR and the induction of emotions. Given the restrictive nature of the fMRI acquisition, it is possible to create naturalistic scenarios that induce emotional states in participants, but it is more challenging to create ones that allow free engagements of the participants.

Thus, better choice of stimuli and better characterization of stimuli properties are necessary for understanding the brain. This involves the use of a big stimuli set, either introducing a great variety in the attributes of no interest (examples include varying the location, visual angle size and contrasts of stimuli), or varying the attributes in a controlled way such as parametrizing them already at the stimuli construction stage (examples include greebles, 


\section{Chapter 8}

Gauthier \& Tarr, 1997, and morphed stimuli, Freedman, Riesenhuber, Poggio, \& Miller, 2001). Efforts have been made in MVPA studies to better control the stimuli, while utilizing representational similarity analysis (RSA) and its ability to examine large amounts of stimuli (Nili et al., 2014). One study used the first approach of introducing big variability. They compared the representation of multiple emotions across modalities, with face, body and voice stimuli. They found that clusters in the medial prefrontal cortex and left STS contained information of emotional categories across these modalities (Peelen, Atkinson, \& Vuilleumier, 2010). Two other studies used the second approach of constructing stimuli. One of them used 6 categories of objects, with the members of each category consistently varying in 9 shapes, thus was able to disentangle the representation of lower-level shapes from the higher-level object categories (Bracci \& Op de Beeck, 2016). The other study examined the passive viewing and grasping of objects, by creating 6 object shapes that varied in size, and varying the grasping action performed by the participants. They observed that the shape and motor aspects were differently represented in ventral and dorsal streams (Fabbri, Stubbs, Cusack, \& Culham, 2016). We used the first approach in our RSA study (Chapter 6), by including 10 action categories, much more than most of the body and action studies, and controlled for the actor identities. Although we tried to quantify the implied motion and valence of the stimuli, these two attributes were only a tiny portion of all quantifiable attributes, and only started to reveal to us the intricacy of bodily action processing in the brain. Better quantifying the features and finding the more relevant ones would remain to be a big challenge for us and all other researchers in the future.

The univariate localization approach relies heavily on the activation shown by a contrast. However, several possible reasons would not produce activation, even if these areas are involved in a task. One reason is related to the "specificity" of the stimulus. For areas that are involved in multiple tasks, a contrast would subtract out the activity "not specific to the stimuli" in these areas, even the area is crucial to carry out a task. The posterior IPS is an example of such areas. It has been thought to be implicated in eye movements, attention, saliency (Corbetta \& Shulman, 2011), accordingly they were activated during most of the experimental tasks when contrasting the baseline. In Chapter 5, indeed what we found there was not the presence of a difference, namely activation, but was the absence of signal difference for upright visible and invisible bodies. Similar to tools, the body stimuli also activate the IPS much more than the face stimuli (see Chapter 7), possibly relating to their ability to induce action representations. Due to the IPS's involvements in multiple tasks, using a functional localizer and imposing specificity to bodies as an object category in the dorsal stream would limit the understanding of the IPS itself, and the bodily action processing. But for creating ROIs and studying the dorsal areas though, this creates troubles. We used tools>baseline to create dorsal ROls in Chapter 5, and compared the activation to bodies>baseline. They turned out to be largely overlapping, although the tool category appeared to show a lateralization to the contralateral hemisphere of the participant's dominant hand (Chapter 5, Figure 2). The posterior IPS is not only involved in attentional mechanisms, but also in stimuli categorization and decision making, single-cell recordings there showed a mixture of sensorimotor and decision-making components (Freedman \& Miller, 2008; Huk, Katz, \& Yates, 2017). In Chapter 6, we observed a cluster there that contained information that correlated to the 10-action-category structure. However, our IPS 


\section{General discussion}

results do not allow us to unequivocally attribute one of the abovementioned functions to the observed data in each experiment. There are several other areas in the brain that are also such multi-tasking areas, including the posterior medial parietal areas, and the medial prefrontal areas. These two areas are part of the default mode network, and even show deactivation comparing to baseline during fMRI tasks (Gusnard, Raichle, \& Raichle, 2001). These areas have also been separately studied in diverse research fields and topics. Given the correlational nature of the fMRI methodology, attributing single functions to these areas under one specific experimental task is dangerous, and under such cases reverse inference is wrong (R. A. Poldrack, 2006; Russell A. Poldrack, 2011). Future research needs to integrate all the evidence from the diverse research fields and topics.

Another reason that an area does not show univariate activation is related to the low temporal signal-to-noise ratio (SNR) and the statistical power of the fMRI method itself. A recent $\mathrm{fMRI}$ task study acquired a large amount of data (100 runs, 500 trials) per participant, and found that $71 \%$ of the brain was time-locked to the task when using all data with the traditional hemodynamic response (HDR) model, some areas show very low but statistically significant percent signal changes. Using two other HDR models, they found even more brain areas were activated during the task. This study demonstrated that the traditional twogamma HDR model was restrictive, and a lot of brain activity was undetected with the amount of data in most of the task fMRI studies (Gonzalez-Castillo et al., 2012). Complicating this matter further, any brain activity that is not time-locked to the predictors e.g. the stimulus-independent thoughts implicating the default mode network would not be detected by the fMRI data analysis. With the low statistical power of $\mathrm{fMRI}$, a multiplecomparison correction is usually applied to the resulting statistical map. When a cluster-size based thresholding method is used, as long as the cluster size does not pass a threshold, even if most of the voxels in the cluster are highly activated, the cluster would be deemed "not activated". The reporting of such thresholded maps also introduced an impression that the activation or brain activity is of "all or none" kind of high certainty, which is not true. To battle this erroneous impression, researchers advocated to represent the statistical significance of a voxel with the map's opacity when reporting fMRI results (Allen, Erhardt, \& Calhoun, 2012), although it is not yet in wide practice.

With the birth of MVPA, multiple studies found areas that contained information of the task or condition, but did not show univariate activation (e.g. Jimura \& Poldrack, 2012; Harrison \& Tong, 2009; Peelen et al., 2010), demonstrating higher sensitivity than univariate methods. We observed a similar discrepancy between these two methods in our data (see chapter 6 , Figure S8): in the areas that correlated with the action category structure (multivariate RSA), some showed strong univariate activation, such as the cluster at right hMT+/EBA; some other areas did not show consistent activation for any of the action categories, such as clusters in the left central sulcus (primary motor area, M1), and left ventral medial prefrontal cortex. These 7T data we acquired had much higher SNR than 3T data, and we acquired 96 trials for each of the 10 conditions. Although the multivariate results of $\mathrm{fMRI}$ data are not yet fully comparable to single-cell recordings (Dubois, de Berker, \& Tsao, 2015), under current $\mathrm{fMRI}$ data acquisition and analysis frameworks, rather than basing inferences on univariate analysis alone, it is crucial to develop and utilize more sensitive methods, such as multivariate analysis, to reduce false negatives. 


\section{Chapter 8}

The abovementioned methodological concerns beg reconsideration of the direction that body- and emotional research goes. As an object category, the processing specificity of bodies in the ventral stream is an interesting research topic. However, body shape processing alone does not cover the other dimensions of bodies involved in action and emotion processing, it is more important to examine beyond the ventral pathway. For the emotion perception research, researchers also adopted the "specificity" emphasis, on emotional stimuli as a whole comparing to non-emotional stimuli, and on specific emotions (e.g. fear) comparing to other emotions. There are ongoing debates about whether "basic emotions" exist (for recent debates see Adolphs, 2017; Barrett, 2017), and whether there are specific brain substrates for each of them. Distinct univariate activation maps between emotions have been used as evidence supporting the existence of basic emotions (e.g. Vytal \& Hamann, 2010). In both object and emotion research, there were arguments about "domain specificity" in absolute or more relative terms (Adolphs, 2017; Kanwisher, 2010). In our data of Chapter 6, we observed different activation sites between positive emotion (happy) and negative emotions (fear, anger, sad), both comparing to non-emotional actions. However, with the multivariate RSA, we did not find positive cluster that discriminated positive from negative valences, but we only found correlating clusters in M1 and the frontal eye field (FEF) when discriminating emotional and non-emotional categories. The M1 and FEF clusters are not likely to be specific to emotion processing per se, but possibly motor and attentional co-varying attributes of the emotional bodily actions. Taking into account the discrepancy between our univariate and multivariate results, assuming or imposing strong/absolute category or function specificity would result in a limited view when interpreting research evidence. Assigning single names for areas displaying a certain phenomenon but not looking beyond them also slows down our understanding of the brain. See the mirror neuron network research for an example (Hickok, 2009; Krakauer, Ghazanfar, Gomez-Marin, Maclver, \& Poeppel, 2017).

The normal brain functions are not only supported by important processing nodes, but also require intact distributed networks involving both cortical, subcortical areas, and white matter tracts that connect them. For example, white-matter damage had been found in patients with severe spatial neglect (Corbetta \& Shulman, 2011), highlighting the importance of intact networks. We observed a network containing categorical structures, larger than the action observation network defined with univariate activation (Chapter 6 ). For each nonemotional and emotional action category, our data showed that the same set of brain areas (ROls defined by univariate activation) were differentially involved in representing them, and also hinted that different areas or networks may be involved. Thus the differential representation of each emotion would less likely to be located in single areas. Future work would be necessary that both focuses in depth on the processing in important areas, and on the information flow in the whole network. However, challenges remain as to determine by which criteria do we treat an area as a functionally involved node (e.g. whether or not showing univariate activation), and how to understand the functions of multi-tasking areas (and their functions in a specific task).

In summary, future research of body and emotion perception would benefit from using large amount of controlled/characterized stimuli (preferably naturalistic stimuli), sensitive analyzing methods, and look in networks beyond absolute definitions of "specificity" with an 
open mind. Implementing all these considerations would greatly broaden the understanding of the brain functions. One recent multivariate decoding study of object perception could be viewed as an exemplar: the study set out to examine whether information orthogonal to the visual object category and identities were present in the ventral pathway, by presenting the monkeys 5760 highly variable but naturalistic image stimuli (categories varied in identity, pose, position and scale, randomly embedded in different naturalistic sceneries). They compared the neural responses in the monkey IT and V4, and found that the information increased progressively for both lower-level attributes and higher-level attributes such as categories, and the information showed broad distributions in IT. Furthermore, they compared the decoding performance using grating patches with much lower variability, and found that the information increase across the areas for naturalistic stimuli was not present, even was reversed for less variable stimuli (Hong, Yamins, Majaj, \& DiCarlo, 2016). This study demonstrated the importance of all the methodological considerations mentioned above, even for areas that was believed to have high category-specificity. We started implementing these considerations in our experimental designs in previous chapters, but there is a long way to go.

\section{The bodies versus the faces, and the involvement of amygdala in emotion processing}

When we acquired functional localizers with $\mathrm{fMRI}$ (Chapter 5, 6, 7, functional resolution 2 $\mathrm{mm}$ isotropic and $1.2 \mathrm{~mm}$ isotropic), we consistently observed in fusiform areas that one body-specific cluster (the FBA) was localized between two face-specific clusters (the FFA) for most of the individual participants, which is consistent with the literature (Peelen \& Downing, 2005; Schwarzlose, Baker, \& Kanwisher, 2005). We explicitly compared the processing of bodies and faces in chapter $\mathbf{2}$ and $\mathbf{7}$. In Chapter $\mathbf{2}$, we found that both the body stimuli, and the emotions expressed by bodies, were processed differently than faces under CFS. The suppression time was longer for bodies in general, and the angry bodies were suppressed shorter than the neutral and fearful bodies, while the angry faces were suppressed longer. In Chapter 7, we saw indeed that the processing faces and bodies during passive viewing involved different brain areas, that the faces activated bilateral amygdalae significantly more than the body stimuli. Between the morphed fearful and happy emotions, we also saw an interaction effect of face/body $x$ morph, in the right lateral prefrontal cortex (pars triangularis), right posterior IPL (superior to EBA), and left lingual gyrus.

Our results of higher amygdala activation for faces than bodies are consistent with previous research (Kret, Pichon, Grezes, \& de Gelder, 2011). However, the different activation of the amygdala for faces and bodies begs further considerations. The activation of the amygdala had been widely found in studies of emotional faces (Dricu \& Fruhholz, 2016; Sergerie, Chochol, \& Armony, 2008), so much so that the presence of the activation has been treated almost as a "biomarker" showing that the brain was involved in emotion processing (or specifically for fear processing), which has been used in reverse inferences and for clinical treatment of depression (see e.g. Nord, Gray, Charpentier, Robinson, \& Roiser, 2017; Plichta et al., 2014, for the reliability of amygdala activation). But the presence of this activation is neither necessary nor sufficient for the involvement of emotional processing. First, the 


\section{Chapter 8}

amygdala does not show consistent activation for emotions expressed in the modalities other than faces. In the literature, A meta-analysis showed that, comparing explicit evaluation of emotions of faces and voices showed activation in the amygdala for the faces, while the explicit evaluation of vocal emotions only did not show amygdala activation (Dricu \& Fruhholz, 2016). In our data, in the amygdala of Chapter 7 where the emotional faces showed higher activity, the activation for most of the emotional body conditions were not different than the baseline. In the chapters $\mathbf{5}$ and $\mathbf{6}$ with body stimuli under fMRI, we did not observe activation for emotional bodies in the amygdala either. Second, previous studies found that the amygdala showed eye-specific effects for the face stimuli. In monkeys, singlecell recording showed specific cells in the amygdala that responded to eye contacts during social interactions (Mosher, Zimmerman, \& Gothard, 2014). For humans, amygdala activation for direct versus averted eye contacts had been found both in a blindsight patient with bilateral V1 lesion and a sighted control group (Burra et al., 2013). For healthy participants, an fMRI study showed higher activity for the eye whites of fearful faces than happy faces (Whalen et al., 2004); and two behavioral studies with CFS found that the eye region alone and lower-level features of the face (e.g. the contrast) could explain the shorter suppression time for fearful faces (Gray, Adams, Hedger, Newton, \& Garner, 2013; Yang, Zald, \& Blake, 2007). Together, these results indicate that the amygdala activation in emotional studies is more likely to be related specifically to face and eye processing, which confounds with its relation to emotion processing in general. A single cell recording study in human amygdala showed the influence of subjective judgments: when participants distinguished between fearful and happy facial stimuli, the activity of the amygdala was mainly driven by the eye regions, and they encoded the subjective judgments of the emotion during incorrect trials (Wang et al., 2014). For processing emotions in other modalities than faces, since previous studies for bodies did find amygdala activation with tasks of 2AFC emotion categorization, color naming and emotion naming (Hadjikhani \& de Gelder, 2003; Sinke, Sorger, Goebel, \& de Gelder, 2010; van de Riet, Grezes, \& de Gelder, 2009), while in this thesis we used passive viewing tasks in all three fMRI experiments and did not find significant activation for emotional bodies in the amygdala, future studies would be necessary to investigate whether and why passive viewing and explicit recognition of bodily emotions differentially engages the amygdala.

\section{Non-conscious body processing and the CFS paradigm}

In Chapter 2-5, we examined the behavioral and neural correlates of non-conscious perception of whole-body emotional postures with the CFS paradigm, and the temporal mechanism of CFS itself.

For the processing of non-conscious emotional bodies, we found that neutral, fearful and angry bodies showed different suppression times, indicating different non-conscious/preconscious processing (Chapter 2); non-conscious fearful bodies specifically showed a facilitation effect for discriminating consciously perceived angry bodies (Chapter 3 experiment 3); non-conscious fearful bodies showed much less activity than conscious ones in ventral body-specific areas (FBA and EBA), but residual activity there still showed an 


\section{General discussion}

inversion effect, i.e. less activity for inverted than upright bodies, which was related to bodyform specific processing. While in the dorsal pathway (areas along the IPS), we found an interaction of body orientation and visibility, this interaction found in pIPS and mIPS ROIs was not present in the aIPS ROI (Chapter 5).

Based on the category-specificity view, the neural correlates we found in the ventral areas were largely consistent with the face processing showed in previous CFS FMRI studies (Fang \& He, 2005; Jiang \& He, 2006), and a behavioral study that showed the presence of the inversion effect for bodies under CFS (Stein, Sterzer, \& Peelen, 2012). Our results in the dorsal areas showed higher independence to awareness for upright bodies, similar to that found for tools (Fang \& He, 2005), although the category specificity for tools is still under debate in the literature (see Chapter $\mathbf{5}$ for a detailed discussion for tool perception in dorsal areas, and see the first section of this chapter for the discussion of category specificity).

However, our data of orientation $\times$ visibility interaction in the dorsal areas hinted an interaction of ventral and dorsal areas. These two streams did not work in isolation, although erroneously believed by a range of researchers. The studies of the visual agnosia patient D.F. that originally established the ventral and dorsal pathways showed ample evidence of both ventral-to-dorsal and dorsal-to-ventral influences (for a review see Milner, 2017). For example, D.F. was unable to pick up an object containing two or three holes by inserting her fingers into the holes, due to the loss of ventral areas providing information for more complex stimuli; but her grips of picking up elongated shapes, a single one or two that were separated $5 \mathrm{~cm}$ apart, were comparable to healthy controls (McIntosh, Dijkerman, Mon-Williams, \& Milner, 2004). When asked to copy lines in different orientations, D.F. was also able to use her hand actions tracing the line, relying on dorsal functions, to compensate her loss of ventral input for performing the task (Dijkerman \& Milner, 1997). Her grasping of elongated objects was particularly relevant to the debate of tool processing in the dorsal pathway. More recently, researchers have been calling the attention not to treat the ventral and dorsal pathways as separate entities, and to study the object perception in the dorsal pathway in more detail (Freud, Plaut, \& Behrmann, 2016).

One more consideration is that, we may need to rethink the role of EBA as a "ventral area". The hMT+ in the lateral occipitotemporal surface of the cortex is involved in processing visual motion, which has direct LGN and pulvinar input that bypass V1 (Benevento \& Rezak, 1976; Sincich, Park, Wohlgemuth, \& Horton, 2004), allowing monkeys subjects and human blindsight patients without $\mathrm{V} 1$ to show residual processing of motion information (e.g. Hervais-Adelman et al., 2015). The EBA is located just adjacent to the hMT+ complex, and often shows overlap to the hMT+ in fMRI data, with voxels showing motion sensitivity (e.g. see Vangeneugden, Peelen, Tadin, \& Battelli, 2014). With such a short distance to hMT+, it is very likely that the EBA also receive inputs bypassing V1. Indeed, activation for bodies in EBA has been found for the patient T.N. with bilateral V1 lesion, under fMRI scan (Van den Stock et al., 2014). A recent DTI and functional connectivity study in healthy participants showed that, although both the EBA and FBA showed stronger DTI connections to the ventral areas, the EBA showed stronger functional connectivity to dorsal areas (Zimmermann, Mars, de Lange, Toni, \& Verhagen, 2017). These data all urge a rethinking of the role EBA plays in visual and action perception. 


\section{Chapter 8}

Our experiments also shed more light on the temporal and spatial mechanisms of CFS. We examined the temporal properties of the flash frequencies (Chapter 4), which were examined only by a handful of studies, and we found the numbers of seen trials was fewer under 4,6 and $8 \mathrm{~Hz}$ than the commonly used $10 \mathrm{~Hz}$. Most CFS studies (including us for most of our experiments) adopted the $10 \mathrm{~Hz}$ frequency introduced by Tsuchiya and Koch (2005), where they found 3 flash frequencies $(3.125,6.25$ and $12.5 \mathrm{~Hz})$ that induced longer suppressions in a separate experiment, and used $10 \mathrm{~Hz}$ in their main experiments. The use of $10 \mathrm{~Hz}$ and the paucity of studies of flash frequencies may also be related to the ease of implementation, because common LCD screens (refresh rate $=60 \mathrm{~Hz}$ ) could present stimuli at $10 \mathrm{~Hz}$ ( 6 frames per stimuli), but not $8 \mathrm{~Hz}$ for example. We used an LCD screen with a refresh rate of $120 \mathrm{~Hz}$ to achieve this ( 15 frames per stimuli). The optimal frequencies we found indicate that the temporal mechanism of CFS involves interacting with the temporal rhythms of spatial attention, probably "jamming" it by the high-contrast noise patterns at its optimal frequency. The attentional sampling rhythm around $7 \mathrm{~Hz}$ itself is a theory re-surfaced only very recently and has been increasingly studied (for a review see VanRullen, 2016). Although changing the flash frequency did not seem to qualitatively change the CFS effect (because we saw a weaker correlation of frequency with visibility, than the correlation of trial order with visibility, which showed most participants steadily seeing more trials as the experiment progressed), the use of CFS in future studies combined with methods of high temporal resolution (EEG/MEG/ECog) may help understand the temporal mechanisms of both CFS and attention.

For the suppression property of CFS, we found the suppression strength was very strong. Previous literature indicated that the interocular suppression happened at V1 or LGN (Blake \& Wilson, 2011), but the consequence of it seemed to be a weaker activity at ventral areas, which in turn indicates that the ventral area involvement is necessary (but not sufficient) for conscious vision. The suppression was so strong that we found no dissociation of behavior to awareness (i.e. floor effects) in two of our experiments in Chapter 3. However, the effect we found in the third experiment of Chapter 3 bore strong resemblance to results obtained in hemianopia patients with $\mathrm{V} 1$ lesion but without blindsight: the classical redundant target effect observed with blindsight patients was not present in those hemianopia patients; instead unconsciously perceived fearful faces facilitated the conscious perception of incongruent stimuli (even in non-emotion tasks), but this effect was not significant when the consciously perceived stimuli was also expressing fear (Bertini, Cecere, \& Ladavas, 2013, 2017). The hypothesized interocular suppression site for CFS in the brain (V1) bore further resemblance to a follow-up study, where inhibiting the occipital cortex (at the EEG cap site of 01 , roughly corresponding to the occipital pole) reversed a classical redundant target effect in healthy participants to a facilitation effect of fear, similar to that observed for hemianopia patients (Cecere, Bertini, \& Ladavas, 2013).

Two insights could be obtained from the resemblance to results of hemianopia patients studies. First, very strong suppression/interference/competition at V1 may not allow enough information to transpire into higher level areas, to induce a behavioral or neural effect. The abovementioned studies first presented to hemianopia patients a range of visual stimuli in the blind field, including dots, emotional/non-emotional faces, geometrical shapes, and tested with visual detection and $2 \mathrm{AFC}$ tasks. After they show that the patients exhibited at- 


\section{General discussion}

chance performance, they proceeded to the main experiments (Bertini et al., 2013, 2017). Since "finding dissociation" is the most important strategy to study unconscious processing, it is crucial to find the right behavioral or neural measure, which is sensitive enough to pick up the dissociation. Thus "finding no effect" should not be used as evidence for the unconscious mechanism "not present". Previous research implicated that the experimental tasks and the participant's response criteria/biases would influence the behavioral outcome. Rams $\varnothing y$ and Overgaard (2004) proposed a four-point graded scale (perception awareness scale, PAS) to measure awareness, which was more informative than dichotomous responses such as $2 \mathrm{AFC}$ or yes/no tasks. Indeed, in a recent study, when a hemianopia patient could be categorized as blindsight based on 2AFC above-chance performance, using the PAS showed that her above-chance responses were associated with degrees of stimuli awareness (Mazzi, Bagattini, \& Savazzi, 2016). However in a study of the famous blindsight patient G.Y., the receiver operating characteristics (ROCs) of his yes/no and 2AFC responses showed a higher sensitivity for 2AFC than the yes/no task, which was different to healthy controls that showed no such dissociations between sensitivities of these two tasks, indicating fundamentally different vision for G.Y. than healthy participants (Azzopardi \& Cowey, 1997). For our case of healthy participants under CFS, and the case of hemianopia patients without blindsight, 2AFC, d' (without constructing the ROC curve) and confidence of detection, and priming of single unconscious stimulus were not sensitive measures, while the redundant target paradigm (be it showing a gain effect for congruent or incongruent conditions), the breaking from CF-suppression, and the fMRI measure in the ventral stream were more sensitive measures. However, these measures are not optimal, in the sense that they only reveal limited information about a few facets of the unconscious mechanism, and do not always work. For example, another study of the hemianopia patients showed no behavioral effect with the redundant target paradigm, but only found an effect in the ERP measure (Cecere, Bertini, Maier, \& Ladavas, 2014). Recent literature had also raised concerns on methodology of finding dissociations in consciousness research, pointing out that partial selection of all trials/participants, or purely a statistical phenomenon coined as "regression to the mean", could result in the appearance of bogus dissociations (Schmidt, 2015; Shanks, 2017). The statistical phenomenon is: as long as two measures are correlated, the random noise would make a measure (be it same or a different kind) taken twice dissociate with each other (Barnett, van der Pols, \& Dobson, 2005). Based on this phenomenon, if one of two measures of behavioral performance (for example 2AFC performance and the confidence) is at chance level, while the other is above chance, it could purely arise from "regression to the mean". In our opinion, analyzing data of all conditions, and measuring the different brain areas at each same time points would largely devoid of the "regression to the mean" problem, while the low sensitivity of the measures themselves is a more troublesome. I believe the non-conscious visual processing mechanisms are so basic and important, that they should be universally present and detectable in every single neuro-typical individual human adult, given a measure that is sensitive enough. The quest for finding the right measure continues.

This leads to the second insight from hemianopia patient studies, and from patient studies in general: multiple inter-individual variabilities are present. Patients have variable lesioned sites, and multiple different lesions could induce the same symptom in those patients, these 


\section{Chapter 8}

lesions all create "one" phenomenon: the loss of conscious vision. However, even for the blindsight patient G.Y., the "loss of conscious vision" is a more complex phenomenon, where under specific circumstances he could still obtain conscious vision in his blind visual field (e.g. afterimages by hemianopic completion, perception of chromatic color), and involved brain reorganizations such as novel pathways not detected in healthy controls (for a review see Silvanto, 2015). Thus the "loss of conscious vision" should in fact involve multiple processing areas and routes, both cortical and subcortical, the utilization of which varies across individuals. We found large inter-individual variability already at the level of the CFsuppression efficacy: the CFS initially worked for most of the participants, but most of them also showed an increase in the number of stimuli they saw. This increase was present in 33 of the 37 participants in the experiment of Chapter 4. Only a few participants had their percepts reliably suppressed during a long experimental period (e.g. 2 hours). We also found inter-individual variability in the affective priming experiment, for the unconscious influence of stimuli across different emotions, observing patterns of individual participants both consistent and opposite to our hypothesis, which led to group-level non-significant effects. We further observed inter-individual variability for the optimal frequencies, the pattern of seen trials across the 9 frequencies we tested (the peaks and troughs) varied across participants. Turning back to the logic of patient studies, researchers first screened patients for consistent behavioral performance or neural lesion locations, and then studied similarities of non-conscious processing among these screened patients, thus controlling to a certain extent the inter-individual variability associated to different lesions, different compensation mechanisms and even neural reorganizations after lesion. Similarly in individual healthy participants, such complex variability of neural organization/utilization may also be present, leading to variability in behavioral performances. With the development of statistical inference methodology, and an increasing emphasis on detailed single-subject fMRI research with large amount of data, for 3T (Braga \& Buckner, 2017; Gordon, Laumann, Gilmore, et al., 2017; Laumann et al., 2015), 7T and higher magnetic fields, it is plausible to study non-conscious visual perception in healthy participants in the way of patient research (or even the monkey research), for example starting by characterizing individual participants and grouping them. This kind of research requires high level of care to details, proficiency in a wide range of methodology, and a large resource of neuroimaging acquisition. The involvements of cortical and subcortical routes to unconscious emotion processing also await further studies.

\section{The challenge of whole-brain group analyses with ultra-high field fMRI}

Since the introduction of BOLD fMRI, the main-stream data analysis strategy for lower-field and lower-resolution whole-brain data (magnetic field $\leq 3 \mathrm{~T}$, voxel size $>2 \mathrm{~mm}$ isotropic) was univariate, based on cognitive subtraction (Friston et al., 1994; Friston et al., 1996), a continuation from the analysis strategy for PET data. The assumption was that, two (or more) cognitive functions share some overlapping non-specific brain mechanisms, and some function-specific brain mechanisms, and the subtraction of activity would reveal the areas that are specific for each function. In each voxel, the parametric estimates for each 


\section{General discussion}

condition were estimated with the general linear model (GL M), and the subtraction was performed. The adjacent voxels in a local area are also assumed to perform similar tasks. To alleviate the low signal-to-noise ratio (SNR) and contrast-to-noise ratio (CNR) of the data, and to account for the different anatomical structures across a group of participants, the data were further spatially smoothed with kernels as large as $12 \mathrm{~mm}$ FWHM in older studies. Even with lower resolution, a typical fMRI dataset contains hundreds of thousands of voxels (about 50K at $3 \mathrm{~mm}$ isotropic resolution, and in our data about 200K at $2 \mathrm{~mm}$ isotropic resolution). The multiple-comparisons problem in the group inference map could then corrected either by false discovery rate (FDR) correction, or by cluster thresholding that assumes two adjacent noise voxels both being randomly activated is less frequent than only one voxel (Forman et al., 1995), re-enforcing the assumption that voxels with similar functions should form spatial clusters. This univariate analysis scheme worked well on lowfield and low-resolution data, typically rendering a dozen or so extensive clusters after the group analysis. Conversely, researchers would look into specific brain areas by ROI analysis, with ROIs either defined anatomically, or defined by a functional localizer.

The advent of 7T functional imaging not only offered higher spatial resolution and specificity, but also a higher CNR for the BOLD signal than 3T (Ugurbil, 2014). These advantages led to precise and fine-grained localization across the brain. In cortical areas previously showing larger clusters under lower resolution, high-resolution data reveal multiple sub-clusters with different functions (Gentile, van Atteveldt, De Martino, \& Goebel, 2017); the subcortical structures that did not give good SNR in lower fields could also be examined (De Martino et al., 2017). Sub-millimeter resolution brought by $7 T$ and higher field $\mathrm{fMRI}$ were also used in studying the functions of the human brain in meso-scale (De Martino et al., 2017).

The advantage of 7T functional imaging could also be seen in our data of Chapter 6 (7T, Figure $\mathbf{S 7}$ and $\mathbf{S 8}$ ). Shown in Figure $\mathbf{5 7}$, the clusters found in the right medial geniculate nucleus and the left substantia nigra located well within the anatomical boundaries of these two structures. Shown in Figure $\mathbf{8 8}$, with a slow event-related design and very short stimuli presentation $(500 \mathrm{~ms})$, the group-averaged percent signal change for fearful body stimuli could reach 6\%; much higher than the percent signal change found in Chapter 5 (3T), 0.65\% (slow event-related design with longer presentation, although with a different experiment paradigm: CFS. See Supplementary data in Chapter 5). One of our participants took part in both experiments of Chapter 5 and Chapter $\mathbf{6}$, where data for the same functional localizer with static images (block design, conditions: faces, bodies, houses, tools, words) were acquired under both $3 \mathrm{~T}$ and $7 \mathrm{~T}$. In the right EBA of that participant, the percent signal changes across the conditions ranged from around $1 \%-3 \%$ under $3 \mathrm{~T}$, and ranged $2 \%-7 \%$ under $7 \mathrm{~T}$.

However, to examine multiple brain areas at a larger spatial scale, especially for the wholebrain analysis, and to draw group inference, utilizing $7 \mathrm{~T} \mathrm{FMRI}$ is not without cost. At the data acquisition level, under the constraints of TR (2000 ms) and good SNR, the smaller voxels led to a smaller spatial coverage than 3T. In our case (Chapter $\mathbf{7}$ and $\mathbf{6}$ ) this results in a 3T coverage of $1549032 \mathrm{~mm}^{3}$ (193629 functional voxels, $2 \mathrm{~mm}$ isotropic), and a smaller 7T coverage of $935396 \mathrm{~mm}^{3}$ but a big increase in voxel number (541317 functional voxels, 1.2 $\mathrm{mm}$ isotropic). 


\section{Chapter 8}

At the univariate data analysis level, the abovementioned whole-brain analysis scheme could not be applied without modification. As the activated clusters became smaller and more focally localized in individual participants, the anatomical inter-individual variability became more detrimental for group inference. In our data in Chapter 6, although spatially normalized to the Talairach space, the location of one participant's PSTS corresponded to another participant's EBA; due to the small cluster size and the high inter-individual variability in the fusiform areas, the fusiform body area cluster being activated in 8 of 10 participants could not be found (survive cluster thresholding) in the group analysis of functional localizers, despite the use of cortex-based alignment. See Figure 2. Moderate spatial smoothing ( $3 \mathrm{~mm}$ FWHM) would not alleviate this problem, while excessive smoothing ( $>6 \mathrm{~mm}$ FWHM) would reduce the high-resolution advantage, and even introduce bias or false positive activation (Sacchet \& Knutson, 2013; Stelzer, Lohmann, Mueller, Buschmann, \& Turner, 2014).

The effect of inter-individual (anatomical) variability on group inference was already present at lower magnetic fields and resolutions, but was much overlooked by the majority of researchers (see Figure $\mathbf{2}$ for an example from our data). Two recently published 3T fMRI studies clearly demonstrated this effect. These two studies sampled the resting-state fMRI data of single participants for hours, and estimated multiple brain networks of functional connectivity. One study found that the default mode network was reliably identified as two separate networks in individual datasets, but the functional correspondence of spatially correspondent areas across participants was poor (Braga \& Buckner, 2017). The other study found that network features reliably defined in individual participants were missing in the group-averaged maps (Gordon, Laumann, Gilmore, et al., 2017).

Two approaches seem to be of some help to alleviate this problem for 7T data. One is a better spatial normalization than aligning to the Talairach or MNI templates. This includes non-linear alignments in the volume space (Andersson, Jenkinson, \& Smith, 2007), and the cortex-based alignment (CBA) in the surface space, aligning either the geometrical (Frost \& Goebel, 2012) or myelin information (Van Essen \& Glasser, 2014). However, apart from the resampling necessary for correcting distortions caused by magnetic field inhomogeneity in the 2D gradient echo EPI data (Polimeni, Renvall, Zaretskaya, \& Fischl, 2017), these two methods introduce a lot of resampling of the data, and are computationally or laborintensive. We utilized the CBA approach for our data, aligning the major gyri and sulci across all participants. Although weighing the alignment by the functional data was also possible, we did not perform this step, as it requires additional tuning of the alignment parameters, and we did not assume that weighing by one specific brain function would generalize to all the other functions. To achieve high-quality gray-white matter boundary segmentation, it took us about 90 hours to manually correct the initial automatic segmentation. The CBA worked well for our univariate analysis, but not for the multivariate analysis. In the univariate analysis though, the CBA was not working optimally for areas with big interindividual variability of locations such as the fusiform and the IPS, consistent with previously shown (Frost \& Goebel, 2012). See Figure S11 of Chapter 6, where the averaged brain curvature was shown. The patchy areas (i.e. bilateral IPS) were those not well aligned after CBA. For non-linear alignments in the volume space, Gordon et al., found that functional task activations robustly found in individual data resulted in very sparse clusters in the 


\section{General discussion}

group-averaged map, despite the utilization of non-linear alignments (Gordon, Laumann, Gilmore, et al., 2017). Also, previous cytoarchitectural studies found that the borders of cytoarchitecture do not correspond well to macroanatomical landmarks (e.g. sulcal contours) (Amunts et al., 1999; Caspers et al., 2006). This indicates that aligning anatomical markers alone would not completely alleviate the problem of inter-individual variability.

Another approach is the ROI approach. Functional localizers-defined ROI were originally used to overcome the anatomical variability across individual participants (Saxe, Brett, \& Kanwisher, 2006). However, the current widely used functional localizers are quite specific, with limited ability of localizing areas across different functional systems (e.g. it's not possible to use a face-vs-scrambled localizer for localizing body-sensitive areas). To localize areas across different systems would then acquire a large battery of specific localizers. Under the high resolution of 7T, ROIs defined by functional localizers are smaller than those found in 3T studies, analyzing only these areas would lose the advantage of large brain coverage of the acquired data. On the other hand, ROIs defined according to currently available anatomical templates are usually very big, and does not accommodate individual anatomical or functional variability. A possible alternative would be using resting-state data, which could capture the brain's ongoing intrinsic activity. The intrinsic activity is stable across different consciousness states (awake, anesthetized, sleep), and form coherent spatial patterns, which is partially constrained by anatomy (does not require monosynaptic connections), partially corresponding to DTI results in humans and tracing results in monkeys (Buckner, Krienen, \& Yeo, 2013; Raichle, 2010). These properties make restingstate data an interesting tool to define functional ROls across the brain for each participant. Numerous parcellation studies have been carried out with resting-state data, and correspondence of these resting-state-data-parcellated areas has been found to areas defined by task fMRI activations (Laumann et al., 2015). Although cortex segmentation is necessary for data preprocessing, and the parcellation is not very stable with limited amounts of resting-state data (e.g. less than $90 \mathrm{~min}$ )(Gordon, Laumann, Gilmore, et al., 2017; Laumann et al., 2015), several studies with less densely sampled individual data (within 15 min of resting-state data) had demonstrated that parcellation of 14 or 17 networks could be estimated for individual participants (Gordon, Laumann, Adeyemo, et al., 2017; Yeo et al., 2011). We successfully defined the default-mode network areas with our $3 T$ resting-state data, with moderate smoothing ( $4 \mathrm{~mm}$ FWHM, see Chapter 7), although we observed that less/no smoothing would create multiple dispersed smaller clusters, which were harder to interpret and select as ROIs (data not shown). Given that under $3 T$ the fractionations of the default mode network was already observed when the amount of data was large (Braga \& Buckner, 2017), with the higher anatomical specificity of 7T high-resolution data, choosing the size and location for the seed region would be more problematic; also large numbers, more localized, and much smaller clusters would need better criteria and strategy when defining ROIs. Combining both functional localizer and resting-state data may help.

Apart from the univariate group analysis scheme that compares the population mean against zero, finding instead how prevalent an effect is in a sample of participants, have been proposed for both univariate and multivariate analyses (Allefeld et al., 2016; Rosenblatt, Vink, \& Benjamini, 2014). However, influence of anatomical inter-individual variability should still be considered when adapting this method to 7T analysis, and new 


\section{Chapter 8}

statistical methods need to be developed, to fully utilize the advantages of ultra-high field fMRI.

Figure 2 (opposite page). The anatomical inter-individual variability in 3T and 7T data (from Chapter 7 and Chapter 6 respectively), and its impact on the spatial overlapping of functional clusters. A. The averaged brain of 14 participants under $3 \mathrm{~T}$, and the averaged brain of 10 participants under $7 \mathrm{~T}$. The inter-individual variability of the anatomical structure was big at the IPS and the fusiform area. B. The probabilistic overlap of the EBA and FBA across the 10 participants under 7T. The contrast was bodies > faces, houses, tools, words, from the functional localizer data, smoothed 3mm FWHM, $p=.001$ uncorrected. The EBA and FBA clusters could be robustly found in all individual participants, but across participants the spatial overlap was only as high as $30 \%$, in the light green voxels that could be seen in the sagittal and transverse view. 
A

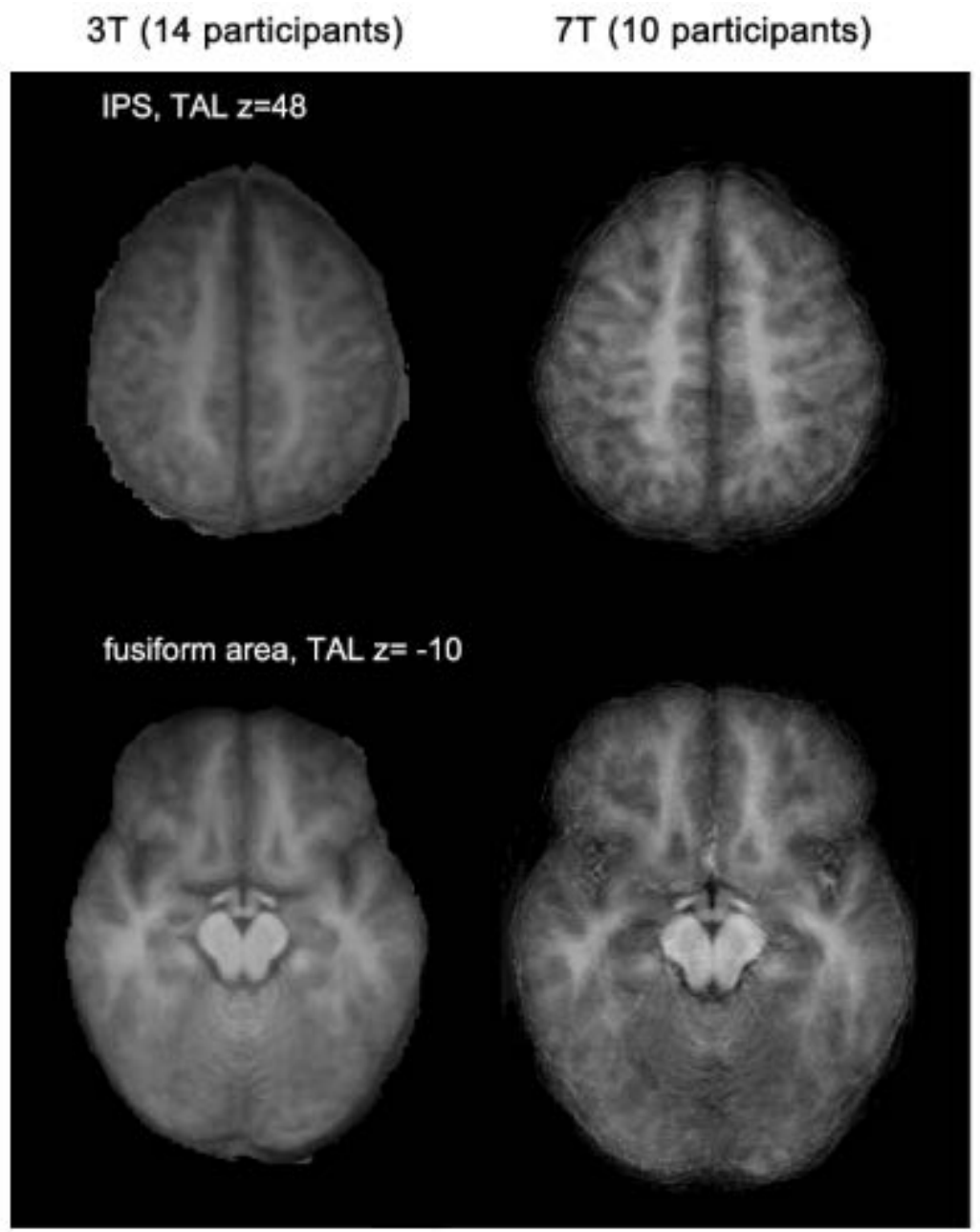

B $7 \mathrm{~T}$ (10 participants)

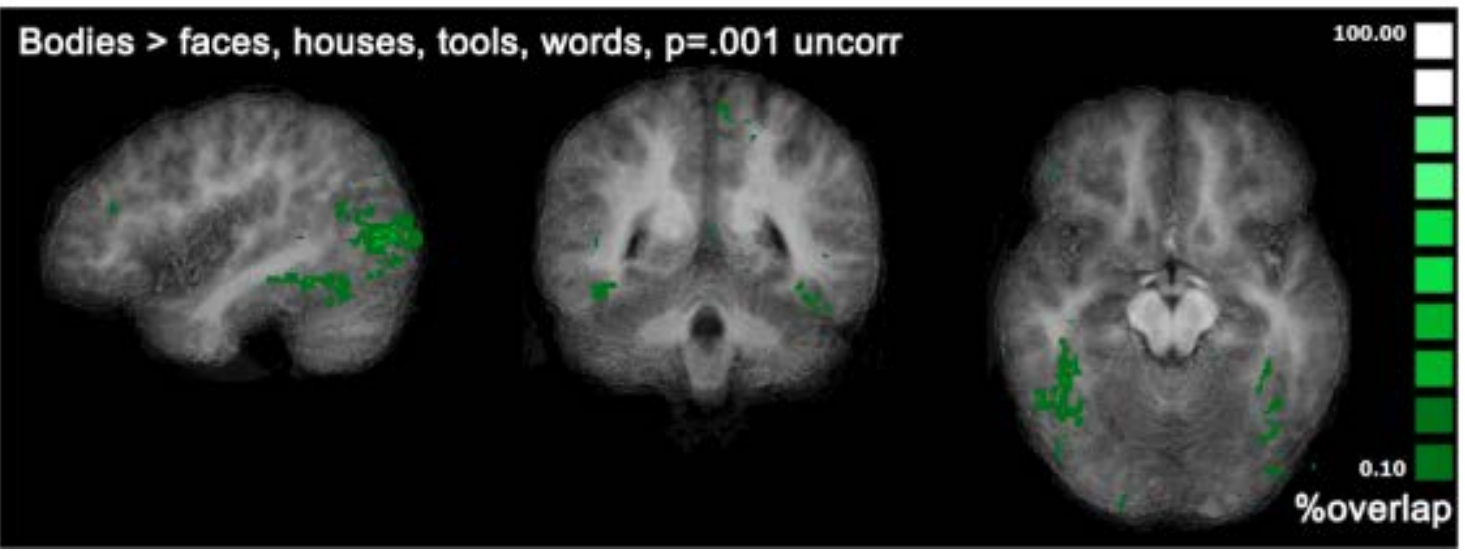




\section{Chapter 8}

\section{Reference}

Adolphs, R. (2017). How should neuroscience study emotions? by distinguishing emotion states, concepts, and experiences. Soc Cogn Affect Neurosci, 12(1), 24-31. doi: $10.1093 /$ scan/nsw153

Allefeld, C., Gorgen, K., \& Haynes, J. D. (2016). Valid population inference for information-based imaging: From the second-level t-test to prevalence inference. Neuroimage, 141, 378-392. doi: 10.1016/j.neuroimage.2016.07.040

Allen, E. A., Erhardt, E. B., \& Calhoun, V. D. (2012). Data visualization in the neurosciences: overcoming the curse of dimensionality. Neuron, 74(4), 603-608. doi: 10.1016/j.neuron.2012.05.001

Amir, A., Lee, S. C., Headley, D. B., Herzallah, M. M., \& Pare, D. (2015). Amygdala Signaling during Foraging in a Hazardous Environment. J Neurosci, 35(38), 12994-13005. doi: 10.1523/jneurosci.0407-15.2015

Amunts, K., Schleicher, A., Burgel, U., Mohlberg, H., Uylings, H. B., \& Zilles, K. (1999). Broca's region revisited: cytoarchitecture and intersubject variability. J Comp Neurol, 412(2), 319-341.

Andersson, J. L., Jenkinson, M., \& Smith, S. (2007). Non-linear registration, aka Spatial normalisation FMRIB technical report TR07JA2. FMRIB Analysis Group of the University of Oxford, 2.

Azzopardi, P., \& Cowey, A. (1997). Is blindsight like normal, near-threshold vision? Proc Natl Acad Sci U S A, 94(25), 14190-14194.

Baker, C. I., Liu, J., Wald, L. L., Kwong, K. K., Benner, T., \& Kanwisher, N. (2007). Visual word processing and experiential origins of functional selectivity in human extrastriate cortex. Proc Natl Acad Sci U S A, 104(21), 9087-9092. doi: 10.1073/pnas.0703300104

Barnett, A. G., van der Pols, J. C., \& Dobson, A. J. (2005). Regression to the mean: what it is and how to deal with it. Int J Epidemiol, 34(1), 215-220. doi: 10.1093/ije/dyh299

Barrett, L. F. (2017). The theory of constructed emotion: an active inference account of interoception and categorization. Soc Cogn Affect Neurosci, 12(1), 1-23. doi: 10.1093/scan/nsw154

Benevento, L. A., \& Rezak, M. (1976). The cortical projections of the inferior pulvinar and adjacent lateral pulvinar in the rhesus monkey (Macaca mulatta): an autoradiographic study. Brain Res, 108(1), 1-24.

Berman, M. G., Park, J., Gonzalez, R., Polk, T. A., Gehrke, A., Knaffla, S., \& Jonides, J. (2010). Evaluating functional localizers: the case of the FFA. Neuroimage, 50(1), 56-71. doi: 10.1016/j.neuroimage.2009.12.024

Bertini, C., Cecere, R., \& Ladavas, E. (2013). I am blind, but I "see" fear. Cortex, 49(4), 985-993. doi: 10.1016/j.cortex.2012.02.006

Bertini, C., Cecere, R., \& Ladavas, E. (2017). Unseen fearful faces facilitate visual discrimination in the intact field. Neuropsychologia. doi: 10.1016/j.neuropsychologia.2017.07.029

Blake, R., \& Wilson, H. (2011). Binocular vision. Vision Res, 51(7), 754-770. doi: 10.1016/j.visres.2010.10.009

Bracci, S., \& Op de Beeck, H. (2016). Dissociations and Associations between Shape and Category Representations in the Two Visual Pathways. J Neurosci, 36(2), 432-444. doi: 10.1523/jneurosci.2314-15.2016

Braga, R. M., \& Buckner, R. L. (2017). Parallel Interdigitated Distributed Networks within the Individual Estimated by Intrinsic Functional Connectivity. Neuron, 95(2), 457-471 e455. doi: 10.1016/j.neuron.2017.06.038

Buckner, R. L., Krienen, F. M., \& Yeo, B. T. (2013). Opportunities and limitations of intrinsic functional connectivity MRI. Nat Neurosci, 16(7), 832-837. doi: 10.1038/nn.3423 


\section{General discussion}

Burra, N., Hervais-Adelman, A., Kerzel, D., Tamietto, M., de Gelder, B., \& Pegna, A. J. (2013). Amygdala activation for eye contact despite complete cortical blindness. J Neurosci, 33(25), $10483-$ 10489. doi: 10.1523/JNEUROSCI.3994-12.2013

Caspers, S., Geyer, S., Schleicher, A., Mohlberg, H., Amunts, K., \& Zilles, K. (2006). The human inferior parietal cortex: cytoarchitectonic parcellation and interindividual variability. Neuroimage, 33(2), 430-448. doi: 10.1016/j.neuroimage.2006.06.054

Cecere, R., Bertini, C., \& Ladavas, E. (2013). Differential contribution of cortical and subcortical visual pathways to the implicit processing of emotional faces: a tDCS study. J Neurosci, 33(15), 6469-6475. doi: 10.1523/jneurosci.3431-12.2013

Cecere, R., Bertini, C., Maier, M. E., \& Ladavas, E. (2014). Unseen fearful faces influence face encoding: evidence from ERPs in hemianopic patients. J Cogn Neurosci, 26(11), 2564-2577. doi: 10.1162/jocn_a_00671

Cohen, L., Dehaene, S., Naccache, L., Lehericy, S., Dehaene-Lambertz, G., Henaff, M. A., \& Michel, F. (2000). The visual word form area: spatial and temporal characterization of an initial stage of reading in normal subjects and posterior split-brain patients. Brain, 123 ( Pt 2), 291-307.

Corbetta, M., \& Shulman, G. L. (2011). Spatial neglect and attention networks. Annu Rev Neurosci, 34, 569-599. doi: 10.1146/annurev-neuro-061010-113731

De Martino, F., Yacoub, E., Kemper, V., Moerel, M., Uludag, K., De Weerd, P., . . Formisano, E. (2017). The impact of ultra-high field MRI on cognitive and computational neuroimaging. Neuroimage. doi: 10.1016/j.neuroimage.2017.03.060

Dijkerman, H. C., \& Milner, A. D. (1997). Copying without perceiving: motor imagery in visual form agnosia. Neuroreport, 8(3), 729-732.

Downing, P. E., Jiang, Y., Shuman, M., \& Kanwisher, N. (2001). A cortical area selective for visual processing of the human body. Science, 293(5539), 2470-2473. doi: 10.1126/science.1063414

Dricu, M., \& Fruhholz, S. (2016). Perceiving emotional expressions in others: Activation likelihood estimation meta-analyses of explicit evaluation, passive perception and incidental perception of emotions. Neurosci Biobehav Rev, 71, 810-828. doi: 10.1016/j.neubiorev.2016.10.020

Dubois, J., de Berker, A. O., \& Tsao, D. Y. (2015). Single-unit recordings in the macaque face patch system reveal limitations of fMRI MVPA. J Neurosci, 35(6), 2791-2802. doi: 10.1523/jneurosci.4037-14.2015

Fabbri, S., Stubbs, K. M., Cusack, R., \& Culham, J. C. (2016). Disentangling Representations of Object and Grasp Properties in the Human Brain. J Neurosci, 36(29), 7648-7662. doi: 10.1523/jneurosci.0313-16.2016

Fang, F., \& He, S. (2005). Cortical responses to invisible objects in the human dorsal and ventral pathways. Nat Neurosci, 8(10), 1380-1385. doi: 10.1038/nn1537

Forman, S. D., Cohen, J. D., Fitzgerald, M., Eddy, W. F., Mintun, M. A., \& Noll, D. C. (1995). Improved assessment of significant activation in functional magnetic resonance imaging ( $\mathrm{fMRI}$ ): use of a cluster-size threshold. Magn Reson Med, 33(5), 636-647.

Freedman, D. J., \& Miller, E. K. (2008). Neural mechanisms of visual categorization: insights from neurophysiology. Neurosci Biobehav Rev, 32(2), 311-329. doi: 10.1016/j.neubiorev.2007.07.011

Freedman, D. J., Riesenhuber, M., Poggio, T., \& Miller, E. K. (2001). Categorical representation of visual stimuli in the primate prefrontal cortex. Science, 291(5502), 312-316. doi: 10.1126/science.291.5502.312

Freud, E., Plaut, D. C., \& Behrmann, M. (2016). 'What' Is Happening in the Dorsal Visual Pathway. Trends Cogn Sci, 20(10), 773-784. doi: 10.1016/j.tics.2016.08.003 


\section{Chapter 8}

Friston, K. J., Holmes, A. P., Worsley, K. J., Poline, J. P., Frith, C. D., \& Frackowiak, R. S. J. (1994). Statistical parametric maps in functional imaging: A general linear approach. Hum Brain Mapp, 2(4), 189-210. doi: 10.1002/hbm.460020402

Friston, K. J., Price, C. J., Fletcher, P., Moore, C., Frackowiak, R. S., \& Dolan, R. J. (1996). The trouble with cognitive subtraction. Neuroimage, 4(2), 97-104. doi: 10.1006/nimg.1996.0033

Frost, M. A., \& Goebel, R. (2012). Measuring structural-functional correspondence: spatial variability of specialised brain regions after macro-anatomical alignment. Neuroimage, 59(2), 13691381. doi: 10.1016/j.neuroimage.2011.08.035

Gauthier, I., \& Tarr, M. J. (1997). Becoming a "Greeble" expert: exploring mechanisms for face recognition. Vision Res, 37(12), 1673-1682.

Gentile, F., van Atteveldt, N., De Martino, F., \& Goebel, R. (2017). Approaching the ground truth Revealing the functional organization of human multisensory STC using ultra high field fMRI. The Journal of neuroscience. doi: 10.1523/jneurosci.0146-17.2017

Gonzalez-Castillo, J., Saad, Z. S., Handwerker, D. A., Inati, S. J., Brenowitz, N., \& Bandettini, P. A. (2012). Whole-brain, time-locked activation with simple tasks revealed using massive averaging and model-free analysis. Proc Natl Acad Sci U S A, 109(14), 5487-5492. doi: 10.1073/pnas.1121049109

Gordon, E. M., Laumann, T. O., Adeyemo, B., Gilmore, A. W., Nelson, S. M., Dosenbach, N. U., \& Petersen, S. E. (2017). Individual-specific features of brain systems identified with resting state functional correlations. Neuroimage, 146, 918-939. doi: 10.1016/j.neuroimage.2016.08.032

Gordon, E. M., Laumann, T. O., Gilmore, A. W., Newbold, D. J., Greene, D. J., Berg, J. J., . . Dosenbach, N. U. F. (2017). Precision Functional Mapping of Individual Human Brains. Neuron, 95(4), 791807 e797. doi: 10.1016/j.neuron.2017.07.011

Gray, K. L., Adams, W. J., Hedger, N., Newton, K. E., \& Garner, M. (2013). Faces and awareness: lowlevel, not emotional factors determine perceptual dominance. Emotion, 13(3), 537-544. doi: $10.1037 / \mathrm{a} 0031403$

Gusnard, D. A., Raichle, M. E., \& Raichle, M. E. (2001). Searching for a baseline: functional imaging and the resting human brain. Nat Rev Neurosci, 2(10), 685-694. doi: 10.1038/35094500

Hadjikhani, N., \& de Gelder, B. (2003). Seeing Fearful Body Expressions Activates the Fusiform Cortex and Amygdala. Current Biology, 13(24), 2201-2205. doi: 10.1016/j.cub.2003.11.049

Harrison, S. A., \& Tong, F. (2009). Decoding reveals the contents of visual working memory in early visual areas. Nature, 458(7238), 632-635. doi: 10.1038/nature07832

Hervais-Adelman, A., Legrand, L. B., Zhan, M., Tamietto, M., de Gelder, B., \& Pegna, A. J. (2015). Looming sensitive cortical regions without V1 input: evidence from a patient with bilateral cortical blindness. Front Integr Neurosci, 9, 51.

Hesselmann, G., Hebart, M., \& Malach, R. (2011). Differential BOLD activity associated with subjective and objective reports during "blindsight" in normal observers. J Neurosci, 31(36), 1293612944. doi: 10.1523/JNEUROSCI.1556-11.2011

Hesselmann, G., \& Malach, R. (2011). The link between fMRI-BOLD activation and perceptual awareness is "stream-invariant" in the human visual system. Cereb Cortex, 21(12), 2829-2837. doi: $10.1093 /$ cercor/bhr085

Hickok, G. (2009). Eight problems for the mirror neuron theory of action understanding in monkeys and humans. J Cogn Neurosci, 21(7), 1229-1243. doi: 10.1162/jocn.2009.21189

Hong, H., Yamins, D. L., Majaj, N. J., \& DiCarlo, J. J. (2016). Explicit information for category-orthogonal object properties increases along the ventral stream. Nat Neurosci, 19(4), 613-622. doi: $10.1038 / \mathrm{nn} .4247$ 


\section{General discussion}

Huk, A. C., Katz, L. N., \& Yates, J. L. (2017). The Role of the Lateral Intraparietal Area in (the Study of) Decision Making. Annu Rev Neurosci, 40, 349-372. doi: 10.1146/annurev-neuro-072116031508

Jiang, Y., \& He, S. (2006). Cortical responses to invisible faces: dissociating subsystems for facialinformation processing. Curr Biol, 16(20), 2023-2029. doi: 10.1016/j.cub.2006.08.084

Jimura, K., \& Poldrack, R. A. (2012). Analyses of regional-average activation and multivoxel pattern information tell complementary stories. Neuropsychologia, 50(4), 544-552. doi: 10.1016/j.neuropsychologia.2011.11.007

Kanwisher, N. (2010). Functional specificity in the human brain: a window into the functional architecture of the mind. Proc Natl Acad Sci U S A, 107(25), 11163-11170. doi: 10.1073/pnas.1005062107

Kanwisher, N., McDermott, J., \& Chun, M. M. (1997). The fusiform face area: a module in human extrastriate cortex specialized for face perception. J Neurosci, 17(11), 4302-4311.

Krakauer, J. W., Ghazanfar, A. A., Gomez-Marin, A., Maclver, M. A., \& Poeppel, D. (2017). Neuroscience Needs Behavior: Correcting a Reductionist Bias. Neuron, 93(3), 480-490. doi: 10.1016/j.neuron.2016.12.041

Kret, M. E., Pichon, S., Grezes, J., \& de Gelder, B. (2011). Similarities and differences in perceiving threat from dynamic faces and bodies. An fMRI study. Neuroimage, 54(2), 1755-1762. doi: 10.1016/j.neuroimage.2010.08.012

Laumann, T. O., Gordon, E. M., Adeyemo, B., Snyder, A. Z., Joo, S. J., Chen, M. Y., . . Petersen, S. E. (2015). Functional System and Areal Organization of a Highly Sampled Individual Human Brain. Neuron, 87(3), 657-670. doi: 10.1016/j.neuron.2015.06.037

Ludwig, K., Kathmann, N., Sterzer, P., \& Hesselmann, G. (2015). Investigating category- and shapeselective neural processing in ventral and dorsal visual stream under interocular suppression. Hum Brain Mapp, 36(1), 137-149. doi: 10.1002/hbm.22618

Mazzi, C., Bagattini, C., \& Savazzi, S. (2016). Blind-Sight vs. Degraded-Sight: Different Measures Tell a Different Story. Front Psychol, 7, 901. doi: 10.3389/fpsyg.2016.00901

McIntosh, R. D., Dijkerman, H. C., Mon-Williams, M., \& Milner, A. D. (2004). Grasping what is graspable: evidence from visual form agnosia. Cortex, 40(4-5), 695-702.

Milner, A. D. (2017). How do the two visual streams interact with each other? Exp Brain Res, 235(5), 1297-1308. doi: 10.1007/s00221-017-4917-4

Mosher, C. P., Zimmerman, P. E., \& Gothard, K. M. (2014). Neurons in the monkey amygdala detect eye contact during naturalistic social interactions. Curr Biol, 24(20), 2459-2464. doi: 10.1016/j.cub.2014.08.063

Nili, H., Wingfield, C., Walther, A., Su, L., Marslen-Wilson, W., \& Kriegeskorte, N. (2014). A toolbox for representational similarity analysis. PLoS Comput Biol, 10(4), e1003553. doi: 10.1371/journal.pcbi.1003553

Nord, C. L., Gray, A., Charpentier, C. J., Robinson, O. J., \& Roiser, J. P. (2017). Unreliability of putative fMRI biomarkers during emotional face processing. Neuroimage, 156, 119-127. doi: 10.1016/j.neuroimage.2017.05.024

Paré, D., \& Quirk, G. J. (2017). When scientific paradigms lead to tunnel vision: lessons from the study of fear. npj Science of Learning, 2(1), 6. doi: 10.1038/s41539-017-0007-4

Peelen, M. V., Atkinson, A. P., \& Vuilleumier, P. (2010). Supramodal representations of perceived emotions in the human brain. J Neurosci, 30(30), 10127-10134. doi: 10.1523/JNEUROSCI.2161-10.2010

Peelen, M. V., \& Downing, P. E. (2005). Selectivity for the human body in the fusiform gyrus. J Neurophysiol, 93(1), 603-608. doi: 10.1152/jn.00513.2004 


\section{Chapter 8}

Plichta, M. M., Grimm, O., Morgen, K., Mier, D., Sauer, C., Haddad, L., . . Meyer-Lindenberg, A. (2014). Amygdala habituation: a reliable fMRI phenotype. Neuroimage, 103, 383-390. doi: 10.1016/j.neuroimage.2014.09.059

Poldrack, R. A. (2006). Can cognitive processes be inferred from neuroimaging data? Trends Cogn Sci, 10(2), 59-63. doi: 10.1016/j.tics.2005.12.004

Poldrack, R. A. (2011). Inferring mental states from neuroimaging data: From reverse inference to large-scale decoding. Neuron, 72(5), 692-697. doi: 10.1016/j.neuron.2011.11.001

Polimeni, J. R., Renvall, V., Zaretskaya, N., \& Fischl, B. (2017). Analysis strategies for high-resolution UHF-fMRI data. Neuroimage. doi: 10.1016/j.neuroimage.2017.04.053

Raichle, M. E. (2010). Two views of brain function. Trends Cogn Sci, 14(4), 180-190. doi: 10.1016/j.tics.2010.01.008

Rams $\varnothing$ y, T. Z., \& Overgaard, M. (2004). Introspection and subliminal perception. Phenomenology and the Cognitive Sciences, 3(1), 1-23. doi: 10.1023/B:PHEN.0000041900.30172.e8

Rosenblatt, J. D., Vink, M., \& Benjamini, Y. (2014). Revisiting multi-subject random effects in fMRI: advocating prevalence estimation. Neuroimage, 84, 113-121. doi: 10.1016/j.neuroimage.2013.08.025

Sacchet, M. D., \& Knutson, B. (2013). Spatial smoothing systematically biases the localization of reward-related brain activity. Neuroimage, 66, 270-277. doi: 10.1016/j.neuroimage.2012.10.056

Saxe, R., Brett, M., \& Kanwisher, N. (2006). Divide and conquer: a defense of functional localizers. Neuroimage, 30(4), 1088-1096; discussion 1097-1089. doi: 10.1016/j.neuroimage.2005.12.062

Saxe, R., \& Kanwisher, N. (2003). People thinking about thinking people. The role of the temporoparietal junction in "theory of mind". Neuroimage, 19(4), 1835-1842.

Schmidt, T. (2015). Invisible Stimuli, Implicit Thresholds: Why Invisibility Judgments Cannot be Interpreted in Isolation. Adv Cogn Psychol, 11(2), 31-41. doi: 10.5709/acp-0169-3

Schwarzlose, R. F., Baker, C. I., \& Kanwisher, N. (2005). Separate face and body selectivity on the fusiform gyrus. J Neurosci, 25(47), 11055-11059. doi: 10.1523/jneurosci.2621-05.2005

Sergerie, K., Chochol, C., \& Armony, J. L. (2008). The role of the amygdala in emotional processing: a quantitative meta-analysis of functional neuroimaging studies. Neurosci Biobehav Rev, 32(4), 811-830. doi: 10.1016/j.neubiorev.2007.12.002

Shanks, D. R. (2017). Regressive research: The pitfalls of post hoc data selection in the study of unconscious mental processes. Psychon Bull Rev, 24(3), 752-775. doi: 10.3758/s13423-016$1170-y$

Silvanto, J. (2015). Why is "blindsight" blind? A new perspective on primary visual cortex, recurrent activity and visual awareness. Conscious Cogn, 32, 15-32. doi: 10.1016/j.concog.2014.08.001

Sincich, L. C., Park, K. F., Wohlgemuth, M. J., \& Horton, J. C. (2004). Bypassing V1: a direct geniculate input to area MT. Nat Neurosci, 7(10), 1123-1128. doi: 10.1038/nn1318

Sinke, C. B., Sorger, B., Goebel, R., \& de Gelder, B. (2010). Tease or threat? Judging social interactions from bodily expressions. Neuroimage, 49(2), 1717-1727. doi: 10.1016/j.neuroimage.2009.09.065

Stein, T., Sterzer, P., \& Peelen, M. V. (2012). Privileged detection of conspecifics: evidence from inversion effects during continuous flash suppression. Cognition, 125(1), 64-79. doi: 10.1016/j.cognition.2012.06.005

Stelzer, J., Lohmann, G., Mueller, K., Buschmann, T., \& Turner, R. (2014). Deficient approaches to human neuroimaging. Front Hum Neurosci, 8, 462. doi: 10.3389/fnhum.2014.00462

Todd, M. T., Nystrom, L. E., \& Cohen, J. D. (2013). Confounds in multivariate pattern analysis: Theory and rule representation case study. Neuroimage, 77, 157-165. doi: 10.1016/j.neuroimage.2013.03.039 


\section{General discussion}

Tsuchiya, N., \& Koch, C. (2005). Continuous flash suppression reduces negative afterimages. Nat Neurosci, 8(8), 1096-1101. doi: 10.1038/nn1500

Ugurbil, K. (2014). Magnetic resonance imaging at ultrahigh fields. IEEE Trans Biomed Eng, 61(5), 1364-1379. doi: 10.1109/tbme.2014.2313619

van de Riet, W. A., Grezes, J., \& de Gelder, B. (2009). Specific and common brain regions involved in the perception of faces and bodies and the representation of their emotional expressions. Soc Neurosci, 4(2), 101-120. doi: 10.1080/17470910701865367

Van den Stock, J., Tamietto, M., Zhan, M., Heinecke, A., Hervais-Adelman, A., Legrand, L. B., . . . de Gelder, B. (2014). Neural correlates of body and face perception following bilateral destruction of the primary visual cortices. Front Behav Neurosci, 8, 30. doi: 10.3389/fnbeh.2014.00030

Van Essen, D. C., \& Glasser, M. F. (2014). In vivo architectonics: a cortico-centric perspective. Neuroimage, 93 Pt 2, 157-164. doi: 10.1016/j.neuroimage.2013.04.095

Vangeneugden, J., Peelen, M. V., Tadin, D., \& Battelli, L. (2014). Distinct neural mechanisms for body form and body motion discriminations. J Neurosci, 34(2), 574-585. doi: 10.1523/jneurosci.4032-13.2014

VanRullen, R. (2016). Perceptual Cycles. Trends Cogn Sci, 20(10), 723-735. doi: 10.1016/j.tics.2016.07.006

Vytal, K., \& Hamann, S. (2010). Neuroimaging support for discrete neural correlates of basic emotions: a voxel-based meta-analysis. J Cogn Neurosci, 22(12), 2864-2885. doi: 10.1162/jocn.2009.21366

Wang, S., Tudusciuc, O., Mamelak, A. N., Ross, I. B., Adolphs, R., \& Rutishauser, U. (2014). Neurons in the human amygdala selective for perceived emotion. Proc Natl Acad Sci U S A, 111(30), E3110-3119. doi: 10.1073/pnas.1323342111

Wegrzyn, M., Riehle, M., Labudda, K., Woermann, F., Baumgartner, F., Pollmann, S., . . Kissler, J. (2015). Investigating the brain basis of facial expression perception using multi-voxel pattern analysis. Cortex, 69, 131-140. doi: 10.1016/j.cortex.2015.05.003

Westfall, J., Nichols, T. E., \& Yarkoni, T. (2016). Fixing the stimulus-as-fixed-effect fallacy in task fMRI. Wellcome Open Res, 1, 23. doi: 10.12688/wellcomeopenres.10298.2

Whalen, P. J., Kagan, J., Cook, R. G., Davis, F. C., Kim, H., Polis, S., . . Johnstone, T. (2004). Human amygdala responsivity to masked fearful eye whites. Science, 306(5704), 2061. doi: 10.1126/science.1103617

Yang, E., Zald, D. H., \& Blake, R. (2007). Fearful expressions gain preferential access to awareness during continuous flash suppression. Emotion, 7(4), 882-886. doi: 10.1037/1528-

3542.7.4.882

Yeo, B. T., Krienen, F. M., Sepulcre, J., Sabuncu, M. R., Lashkari, D., Hollinshead, M., ... Buckner, R. L. (2011). The organization of the human cerebral cortex estimated by intrinsic functional connectivity. J Neurophysiol, 106(3), 1125-1165. doi: 10.1152/jn.00338.2011

Zhang, H., Japee, S., Nolan, R., Chu, C., Liu, N., \& Ungerleider, L. G. (2016). Face-selective regions differ in their ability to classify facial expressions. Neuroimage, 130, 77-90. doi: 10.1016/j.neuroimage.2016.01.045

Zimmermann, M., Mars, R. B., de Lange, F. P., Toni, I., \& Verhagen, L. (2017). Is the extrastriate body area part of the dorsal visuomotor stream? Brain Struct Funct. doi: 10.1007/s00429-0171469-0 
Chapter 8 
Valorization 


\section{Addendum}

In this thesis, we explored the mechanisms of emotion and human body action processing, under conscious and non-conscious conditions, and with the use of virtual reality (VR). In studying the non-conscious processing of bodily emotions, we also compared our results from healthy participants under the CFS paradigm, to patient groups including blindsight, neglect, and hemianopia without blindsight. We explore the possible influence of our research in three domains: the general public, specific groups of users (especially the patients with visual cortex lesion), and the scientific researchers using fMRI as a tool.

\section{For the general public}

Consciousness is a phenomenon that all of us experience in everyday lives. Even in mundane activities such as non-REM sleeping each night, we transit from a conscious state into a nonconscious state in sleep. When our body parts are in the colliding trajectory of obstacles such as furniture, we move to avoid them without consciously thinking how to carry out the actual movements.

Throughout the history, consciousness and the human brain in general have been attracting people's fascination, which not only motivated scientific researchers to perform various studies, but also stimulated the imaginations of the general public. Brain, consciousness, VR and artificial intelligence (Al)-related themes have been continuously and increasingly featured in numerous sci-fi novels and movies, from the very early Frankenstein (1918), to the more recent Ghost in the shell (1989), Ex machina (2015), and Dan Brown's new novel Origin (2017).

In the current society with more electronic devices, VR and Al have become increasingly accessible. For VR (and the related augmented reality), more and more first-person perspective video games support 3D viewing, effectively creating VR experiences, and large companies such as Google and Facebook (which acquired Oculus) has been actively developing easy-to-use VR and AR technologies. For Al, there are intensive discussions about whether Al algorithms could be conscious, whether they can make decisions, and whether they will take people's jobs soon.

Despite the enthusiasm and increasing discussion in the general public, and the development of the human brain science field for more than 100 years, the general public still holds numerous misconceptions about the brain. Some information is outdated, and some is plainly wrong. For this current situation, both the scientists and the mass media in the general public are responsible.

From the scientist side, the latest brain science results were not disseminated quickly and effectively. We are in the fast-developing subfield of cognitive neuroscience, with fMRI as one of the major research tools. However it is in 1993 that the fMRI technique was developed; and only from the $21^{\text {st }}$ century on, the multivariate-pattern analysis (MVPA, or machine learning. The RSA method in Chapter 6 is of this category) became more and more used in brain research. With such a short history, cognitive neuroscience has not got enough time to widely enter textbooks for the general public. Only a small part of undergraduateand high-school-level textbooks contained information about cognitive neuroscience, while 
most others contained mainly neurobiological (cellular and molecular) information. Also, these neuroscience-related contents are usually from studies done more than 40 years ago. In my own experience, I was trained in basic medical sciences and neurobiology as an undergraduate student, not much cognitive-neuroscience contents were included in my textbooks. Although studies of blindsight was already established in the 1970s, I still remember the shock, when I was told by the BBC documentary Brain Story (2000), that the conscious visual information is only a portion of the visual information received by us every day, while a large portion of the information is unconscious. Another problem is, not all neuroscientists are informed of the most up-to-date knowledge, which includes the methodological knowledge of their tools (e.g. proper fMRI data analysis), the knowledge of other subfields (e.g. about other brain areas and network systems), and the knowledge within their own subfield. This fact has led to the current discussions of a "replicability crisis" in the cognitive science field; we also discussed some other implications of it in Chapter 8. It is worth mentioning though, that the scientific community itself is constantly undergoing scientific debates, and is constantly developing and self-adjusting scientific theories. No absolute "truth" exists, which is especially true for the newest findings.

From the mass media side, whenever a study with potential social relevance is published, they tend to look for eye-catching titles, to over-interpret and over-generalize the research results (e.g. treating significant but small-effect-size results as having big effects), or they simply do not correctly understand concepts, details, and the research results. For example, the blindsight-related research by Prof. Beatrice de Gelder (the current PhD candidate's supervisor) was dubbed the "sixth sense", featured in a popular documentary Through the wormhole (2011), which was hosted by the famous actor Morgan Freeman. In the blindsight phenomenon, one side of some patients' primary visual cortices was destroyed during stroke, leading to a blindness of one half of the visual field contralateral to the lesion, in both eyes. However in this documentary, the narration got this information wrong, by stating that the "left/right eye" was not able to see, instead of the "left/right visual field".

Therefore, on one hand, although the general public is eagerly in need to be informed about the latest brain (cognitive) science developments, people could not discern, and do not have an easy access to many reliable information sources (scientists and mass media). On the other hand, disseminating latest and correct scientific information to the general public is also a demanding task for the scientists. As a result, brain-related myths have been abundant in the public mind for a long time, accepted by most people without further questioning, such as "only $10 \%$ of the brain is used", and "the left and right laterality predicts whether you are good at logic, or art". Various recreational games/apps were also created, based on these myths (including laterality-related ones). They boast to boost your cognitive abilities after you play them, without the support of any evidence in relevant research fields (See e.g. Kable et al., 2017).

To deal with this situation, first of all, we should de-mystify the brain, and debunk the wrong concepts in the public mind. Take brain laterality for example, although previous fMRI research proposed lateralized univariate activation for face processing (in the right fusiform area), visual word form processing (in the left visual word form area), language processing (in the inferior frontal lobe of the left hemisphere), and tool perception (in the contralateral 


\section{Addendum}

side of the dominant hand, which we also observed in Chapter 5, see Figure $\mathbf{2}$ in that chapter), these lateralization were relative, and studies did show activated clusters in the other side now and then. Moreover, single-cell spikes are not fully correlated with univariate activation; recent MVPA studies also showed that, outside the activated voxels, relevant information could be recovered from voxels that does not activate above baseline. This indicates that univariate activation could not yet serve as the sole indicator of a brain area participating in certain processing (See a more detailed discussion in Chapter 8), and laterality is not an absolute concept. Whenever I see a laterality-myth-related post is being retweeted in the social media, I would/will continue to debunk it, and update the audience with the information above.

We should also routinely introduce to the general public our research results, and new exciting results from other researchers. As a starting cognitive science researcher, and also as a professional computer graphics painter with an outreaching audience in social media, this offered me additional opportunities to disseminate my research, and brain-science related facts. I would represent scientific information in a more accessible drawing style, as what I did for a talk by Prof. de Gelder in Japan, 2016 (See Figure 1). When the research article about CFS under fMRI (Chapter 5) was published, I wrote a 7000-word article in the Chinese social media Weibo (January 2018, https://www.weibo.com/ttarticle/p/show?id=2309404197514260695242, which received 955 retweets, 65 comments, 618 faves), describing our research, and previous research results that led to our study. The concepts mentioned there included attention, action perception, the brain, ventral/dorsal pathways, category-specific areas, $\mathrm{fMRI}$, the general linear model (GLM) and MVPA, the intraparietal sulcus, extrastriate brain area, the MT+ complex, the CFS paradigm. To explain these concepts, I drew simple schematic pictures (See Figure 2). I also emphasized how our knowledge of the human brain is gradually built up by numerous studies, each of which tackled only a very small research aspect, and moved the knowledge boundary a little bit further. Up to the point of writing the current chapter, this article has been read for 204045 times. I received comments from the audience, that the article is "easy to understand" for them, that they are very intrigued by our research and the brain research in general, and would like to learn more in the future. Some audience also expressed that before reading this article, they did not realize the bit-by-bit nature of the real research process.

These comments made me realize and believe, that apart from disseminating scientific information and latest research results, it is more important for researchers to show the audience how proper research is done from a researcher's perspective, and what evidence and thinking process are necessary to lead to a conclusion. For a certain piece of research result, we should provide the general public the most essential but rigorous reasoning chain to understand that result. Although not everyone in the general public is well armed with the critical thinking ability, showing them the actual logic links would give them examples to refer to, and to some extent guard them from easily believing a single piece of information without questioning. 

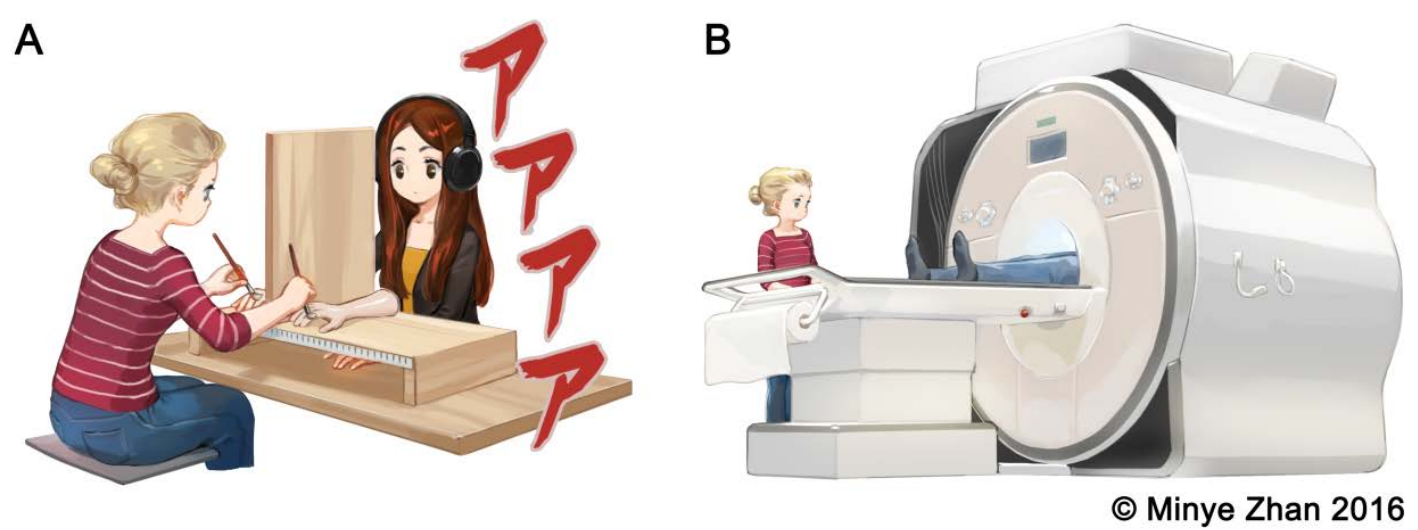

Figure 1. Pictures painted by the current PhD candidate, for the presentation of Prof. Beatrice de Gelder (the current PhD candidate's supervisor), Japan, 2016, in the "manga style" to be more accessible for the Japanese general audience. A. Tahnée Engelen (the current PhD candidate's colleague) performing the rubber-hand experiment on Marta Poyo Solanas (another colleague), while presenting sounds with valence. B. Tahnée Engelen putting a participant into the Siemens 3T Prisma Fit scanner, Scannexus, Maastricht.

A
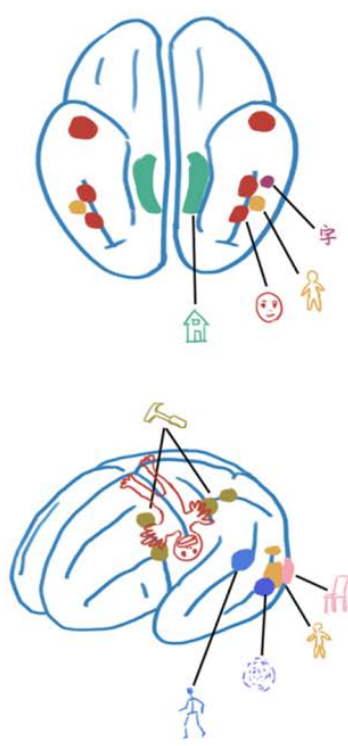

B

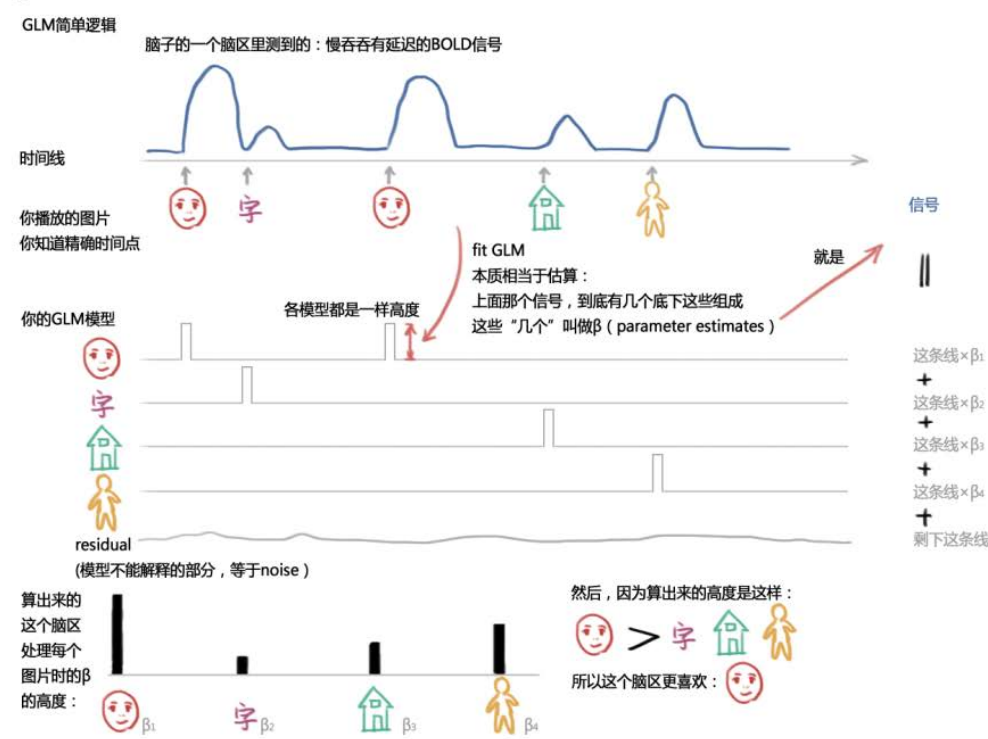

Figure 2. Schematic drawings to explain scientific concepts to the general audience, in an article in the Chinese social media Weibo, about our research (Chapter 5). A. The category-specific brain areas. B. The general logic of GLM (general linear model) analysis in fMRI data.

We also disseminated our research to the public through talks in an art gallery. In an event modulated by Prof. de Gelder (February 2018, http://fundamentalresearch.org/2018/01/18/drawing-new-machine-age/), I was invited to have a conversation with the artist Amelie Bouvier, to discuss the use of "drawing" in both art and brain science. When interacting with the audience, I talked about concepts including unconscious processing, machine learning, peri-personal space, and extension of limbs by tools. While keeping in mind my realization from the comments of the social media, I emphasized the subjectivity of the scientific research, e.g. the interpretation of a same result 


\section{Addendum}

would be different by different researchers, and the same research group would update (and even radically change) their understandings across years. I also emphasized that the results we could see are determined and limited by our tools, a direct example would be the different resolutions under $3 \mathrm{~T}$ and $7 \mathrm{~T} \mathrm{FMRI}$.

These interactions with the general public stimulated interests and enthusiasm to the brain science, and more such interactions would be necessary. I would continue interacting with the general public, whenever there is an opportunity.

\section{For specific groups of users (patients with visual cortex lesion)}

In Chapter $\mathbf{3}$ of this thesis, we compared and discussed the similarities/differences of results obtained in healthy participants under the CFS paradigm, to blind patients with primary visual cortex lesion, which included blindsight patients who could perceive and react to events (although at a degraded level) in their blind visual field, and hemianopia (cortically blind in one visual field) patients without blindsight. Our CFS results bore more resemblance to the results from hemianopia patients without blindsight. Cerebrovascular accidents (strokes) are among the major causes for disability these days according to WHO (http://www.who.int/healthinfo/statistics/bod_cerebrovasculardiseasestroke.pdf), which impose blindness and disabilities to people when the affected sites are the primary visual cortex. It has been advocated that rehabilitation training would reduce the blind visual field to some extent, and trainings could potentially improve the visual abilities of the blindsight patients. Since the majority of the hemianopia patients are without blindsight, if a link of mechanisms between CFS and this kind of patients could be established, it would be possible to train healthy participants to utilize the unseen visual information suppressed by CFS, and later transfer the training to the group of hemianopia patients. To establish a link of the mechanisms, further research is needed.

In Chapter $\mathbf{7}$ of this thesis, we examined the perception of emotional faces and bodies both before and after a domestic-violence VR scenario. The effect of this scenario was previously examined in a group of domestic violence offenders (Seinfeld et al., 2018). In normal male participants, we found that the VR experience induced a reduction of activity specific for fearful faces, in the fMRI run right after it. This indicated that the VR scenario is able to have impacts on people's thinking. Therefore, specific VR programs could be created to influence certain groups of users, for example changing people's perspectives. We should also realize at the same time, in the current society with increasing access to VR experiences, this ability of influencing people should not be misused, and the effect of commercial VR software should be evaluated more carefully.

\section{For the scientific researchers using fMRI as a tool}

As mentioned earlier, there are heated discussions about a "replicability crisis" in the scientific community. This has been a recurring discussion in the cognitive science, particularly in the community that uses fMRI as a tool, where misuses of statistical inference 
were abundant (Eklund, Nichols, \& Knutsson, 2016). And already in the preprocessing stage, the flexibility for the researcher is huge, that multiple preprocessing options are routinely chosen by different researchers (Carp, 2012).

In the fMRI data preprocessing pipeline, I believe that the alignment of functional runs to each other is of the utmost importance. This is especially true for ultra-high field studies with very high functional resolutions (below $1.5 \mathrm{~mm}$ isotropic), including studies trying to analyze activity in different cortical layers. In such studies, a misalignment of two functional runs would make a same piece of cortex tissue ending up in different locations across runs, reducing the specificity and credibility of the results derived from the data.

Although of utmost importance, the alignment of functional runs received relatively little attention. The anatomical image (T1-weighted) is usually with high resolution, while the functional image (T2*-weighted for BOLD images) is usually with much lower resolution, blurry, and with inverted image intensity comparing to the anatomical ones. When using a wide variety of $\mathrm{FMRI}$ data processing packages, most researchers align each functional runs to a certain anatomical run (and if necessary align multiple anatomical runs to each other across sessions), hoping that consequently the functional runs would in this way be aligned with each other. This usual procedure requires the alignment of two images that look very much different. When using this procedure, most of the researchers use automatic aligning algorithms provided by the software packages. One alignment algorithm with better performance (boundary-based registration, BBR) calculates the boundaries of different tissue types, and align these boundaries of the functional image to the anatomical image (Greve \& Fischl, 2009). This and other alignment algorithms usually operate at the wholebrain level. Whether or not using BBR, after alignment is performed, most packages show the user a boundary of the functional image overlaid onto the anatomical image, which looks roughly fitting each other, and gives the impression that the alignment is successfully done.

However, for gradient echo EPI images, image distortion in the encoding directions is inevitable. Even with top-up distortion correction with scans of reversed encoding directions, distortions in different brain areas are different, which varies across runs due to the participant's head-position change in the scanner, and usually cannot be completely removed. This situation cannot be handled by BBR and other algorithms, thus requires the researcher to decide which specific area to align across runs, to implement manual alignments, and calls for careful visual inspection across different functional runs to check alignment quality (which few researchers actually do, to my knowledge). When manual alignment is performed, each time aligning the functional runs to the anatomical run will introduce human error, which accumulates across the functional runs.

To deal with this problem, for all the fMRI studies in the current thesis, we used a manual work-around aligning method in BrainVoyager, where we explicitly align the different functional runs to one of them. With this method, the image modalities (appearances) are the same across runs, and tiny translations/rotations could be observed with visual inspection, and be dealt with (to the precision of 0.1 unit translation/rotation that is operative in BrainVoyager). This method requires only one anatomical run, and could deal 


\section{Addendum}

with across-session functional run alignments. Thus it is not necessary to acquire multiple anatomical runs across different sessions, which in turn saves scanning resources. For our data, we actually checked the alignments across runs until our satisfaction is reached, before proceeding to further analysis. With this method, we observed more robust activation in our data, than when processing with the traditional automatic/manual alignment methods.

We included the manual for our alignment method here. We hope that this will help our colleagues to achieve better alignment, save scanning resources, and at least we hope it would raise the awareness for checking functional run alignment quality.

\section{References}

Carp, J. (2012). On the plurality of (methodological) worlds: estimating the analytic flexibility of FMRI experiments. Front Neurosci, 6, 149. doi: 10.3389/fnins.2012.00149

Eklund, A., Nichols, T. E., \& Knutsson, H. (2016). Cluster failure: Why fMRI inferences for spatial extent have inflated false-positive rates. Proc Natl Acad Sci U S A, 113(28), 7900-7905. doi: 10.1073/pnas.1602413113

Greve, D. N., \& Fischl, B. (2009). Accurate and robust brain image alignment using boundarybased registration. Neuroimage, 48(1), 63-72. doi: 10.1016/j.neuroimage.2009.06.060

Kable, J. W., Caulfield, M. K., Falcone, M., McConnell, M., Bernardo, L., Parthasarathi, T., . . . Lerman, C. (2017). No Effect of Commercial Cognitive Training on Brain Activity, Choice Behavior, or Cognitive Performance. J Neurosci, 37(31), 7390-7402. doi: 10.1523/jneurosci.2832-16.2017

Seinfeld, S., Arroyo-Palacios, J., Iruretagoyena, G., Hortensius, R., Zapata, L. E., Borland, D., ... Sanchez-Vives, M. V. (2018). Offenders become the victim in virtual reality: impact of changing perspective in domestic violence. Sci Rep, 8(1), 2692. doi: 10.1038/s41598-018-19987-7 


\section{BVQX fmr-fmr manual alignment workaround}

Minye Zhan 2016. Updated 2018.

\section{- $\quad$ The basic idea}

The normal procedure of aligning functional runs is to align individual fmr files to the same anatomical vmr file. Since the fmr files and vmr files have opposite image intensities, and usually have different distortions, after aligning a few runs, the fmr files may end up with very big misalignments with each other.

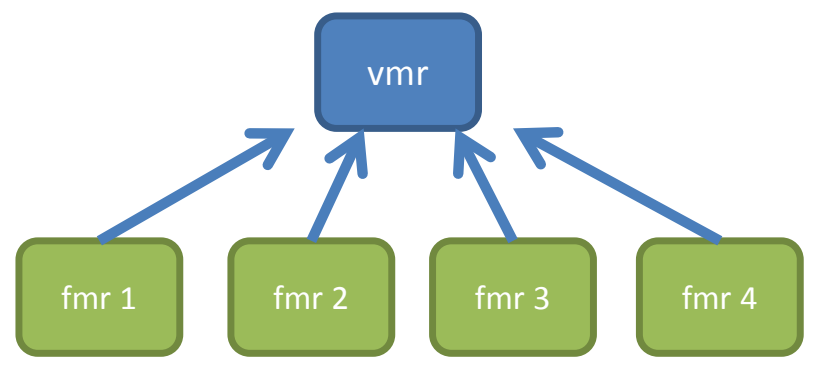

An fmr-fmr alignment is more desirable. Because it doesn't have the inversed intensity any more, it's much easier to spot small differences between similar images, especially when you do manual alignments. However this option is not available in BrainVoyager (in BrainVoyager version 20 and above it offers the option of vtc-vtc alignment though).

Within the 3D motion correction options for fmr files, you can always perform the motion correction with a reference volume from another run, which is a form of automatic fmr-fmr alignment. However this only works if the participant did not move too much between runs. It will fail if participant moved a lot, and it doesn't work for across-session alignments.

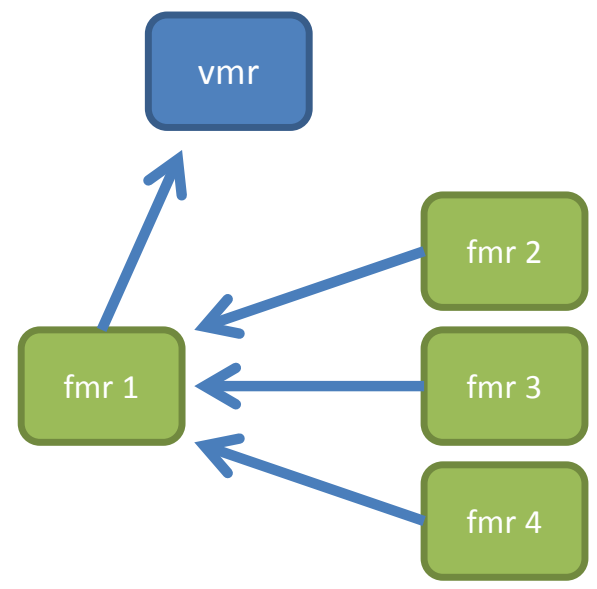

Thus we propose a workaround method for manual alignment. The idea is to first align one of the fmrs to an anatomical vmr, and then create a vtc of the fmr in native space, and save it as a vmr file. This vmr file then has the same intensity as all the other fmrs. With the original vmr's POS information, this newly-created vmr then serves as a target/dummy for subsequent alignments.

\section{Advantages:}

fmr files are similar, thus easier to align between themselves. You can easily do sub-milimeter alignments manually.

Doesn't need to scan multiple anatomical files across different sessions. 
- $\quad$ Procedure

1. Align one of the functional run (.fmr) to the anatomical .vmr (native space), with the standard procedure.

2. Create a .vtc from the aligned .fmr (native space).

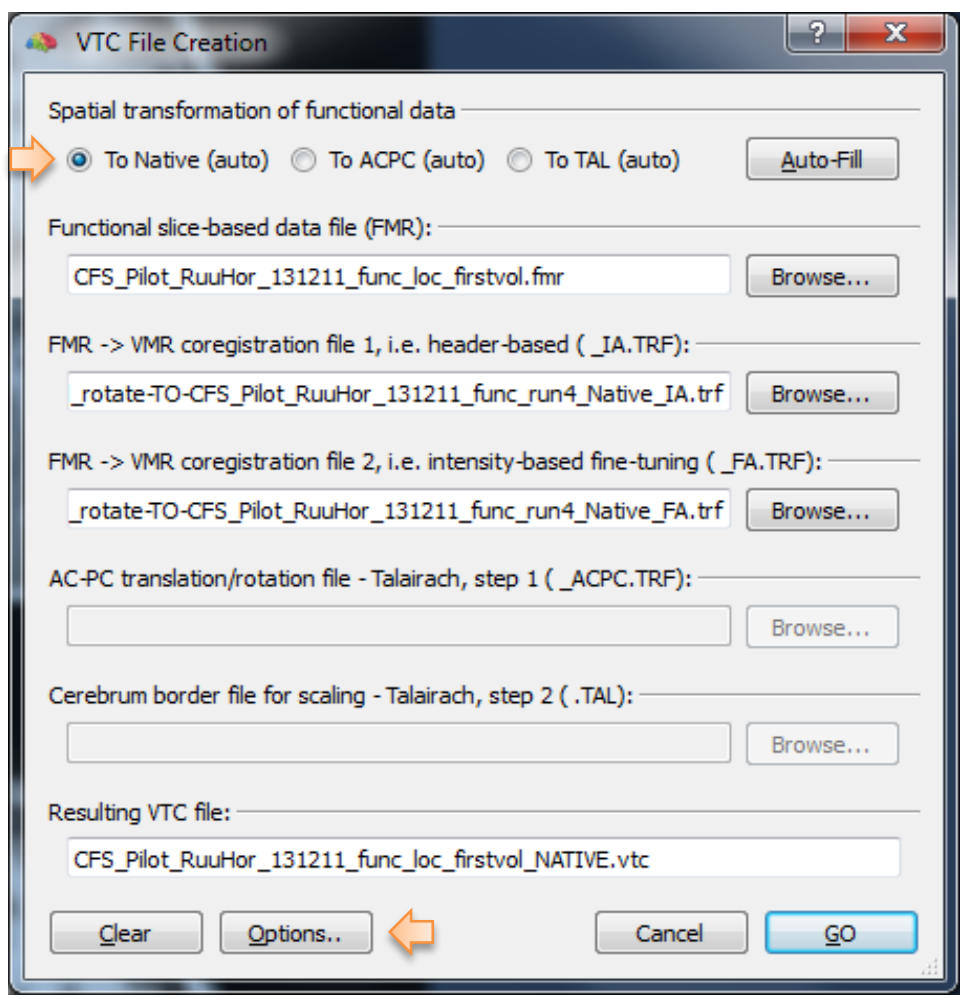

Use the same resolution relationship to the anatomical file as that of your data, and use Sinc interpolation to preserve more details

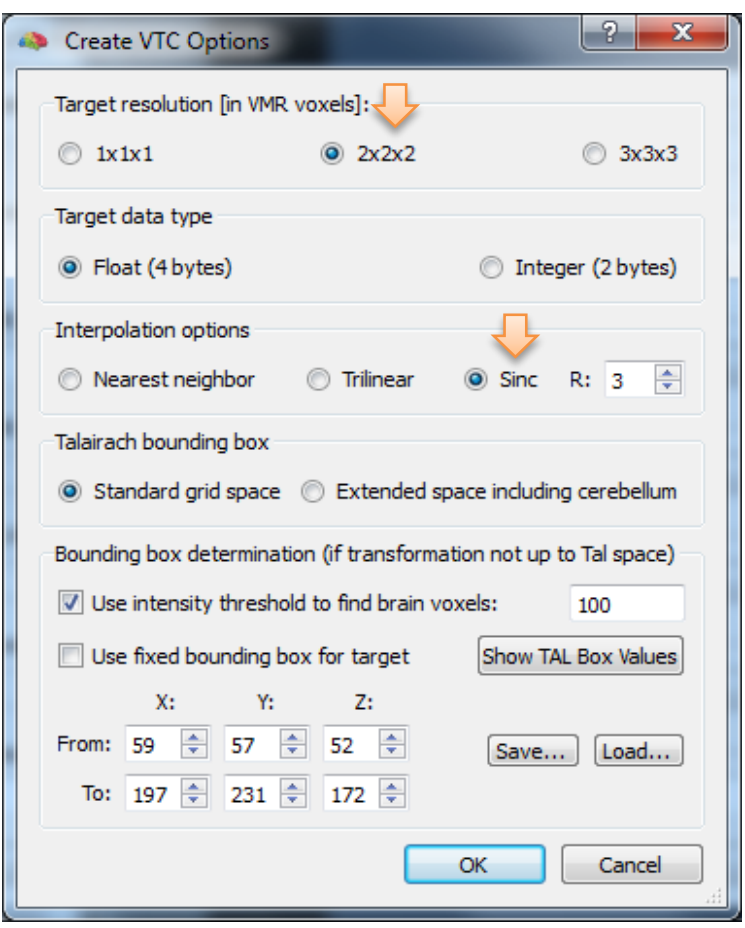

\section{Link the new .vtc to the anatomical .vmr, and click Show VTC Vol}

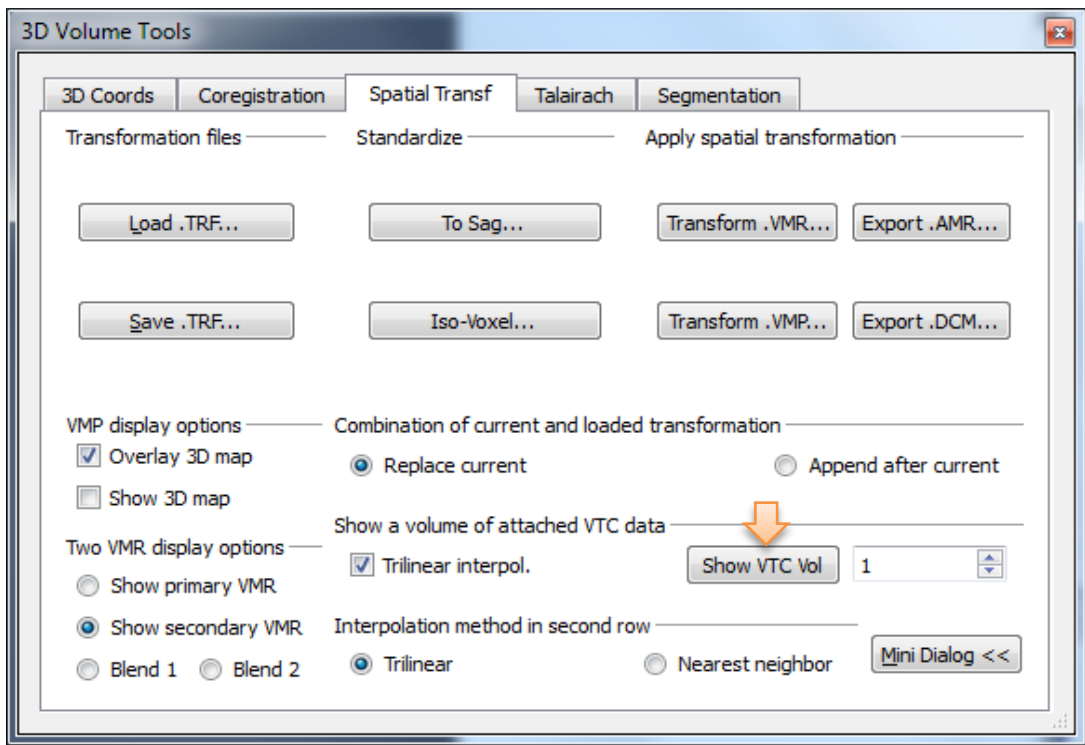

Then save secondary VMR. This new .vmr serves as the target/dummy .vmr for the subsequent alignments.

\begin{tabular}{|c|c|c|}
\hline File & Analysis Options Volumes & Meshes Scer \\
\hline $\mathbb{B}$ & Create Project Wizard... & \\
\hline 긴 & New Project... & $\mathrm{Ctrl}+\mathrm{N}$ \\
\hline 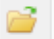 & Open... & $\mathrm{Ctrl}+\mathrm{O}$ \\
\hline \multirow[t]{6}{*}{ 圆 } & Save & $\mathrm{Ctrl}+\mathrm{S}$ \\
\hline & Save As... & \\
\hline & Close & Ctrl+W \\
\hline & Load Secondary VMR... & \\
\hline & Save Secondary VMR... & \\
\hline & Load VTC Mask As Secondary VI & \\
\hline
\end{tabular}

Open the newly created target vmr, and check its properties. Make sure it has the same resolution as the original anatomical vmr (1x1x1 $\mathrm{mm}^{3}$ here), native space, verified:

\section{Reference space}
Unkown
(C) Native
ACPC
Talairach

Voxel resolution (Dicom/TAL axes)
$\square \mathrm{X}: 1.000$
Y: 1.000
Z: 1.000
$\square$ In TAL mm
V Verified

The modifications will take effect only after you save the target vmr. 
4. Align each .fmr (including the $1^{\text {st }} . \mathrm{fmr}$ again) to the newly created target VMR.

Uncheck "invert intensities", and run IA.

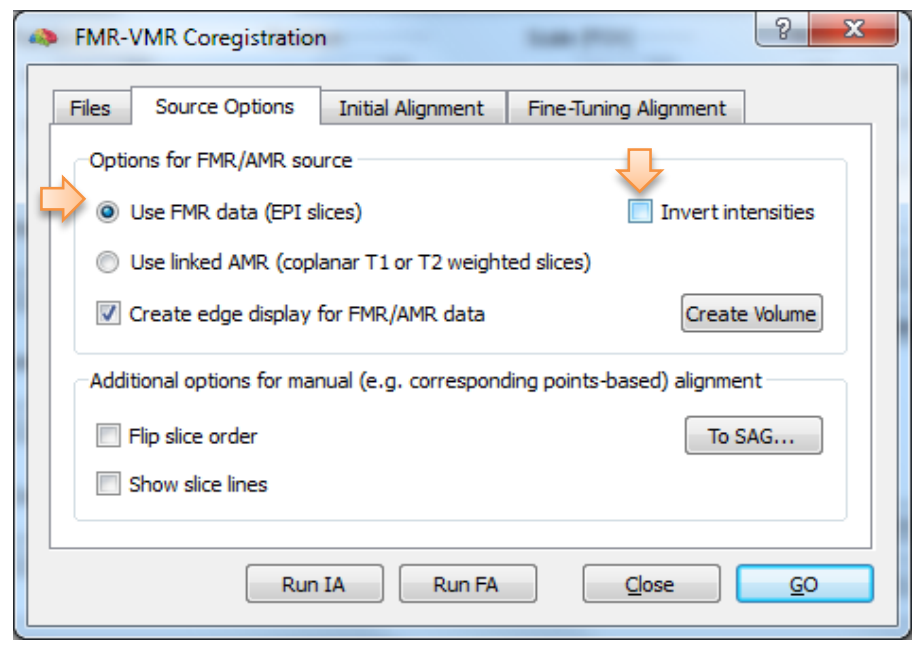

No need to invert intensities, since we are now aligning $\mathrm{T} 2 *$ images to $\mathrm{T} 2 *$ images (fmr-to-fmr), no longer T2* images to T1 images (fmr-to-vmr).

Make sure it uses FMR data, not AMR data for alignment.

The default IA option uses the POS info (info about the participant position in the scanner) to align the fmr to the vmr. The newly created target .vmr doesn't have its own POS info.

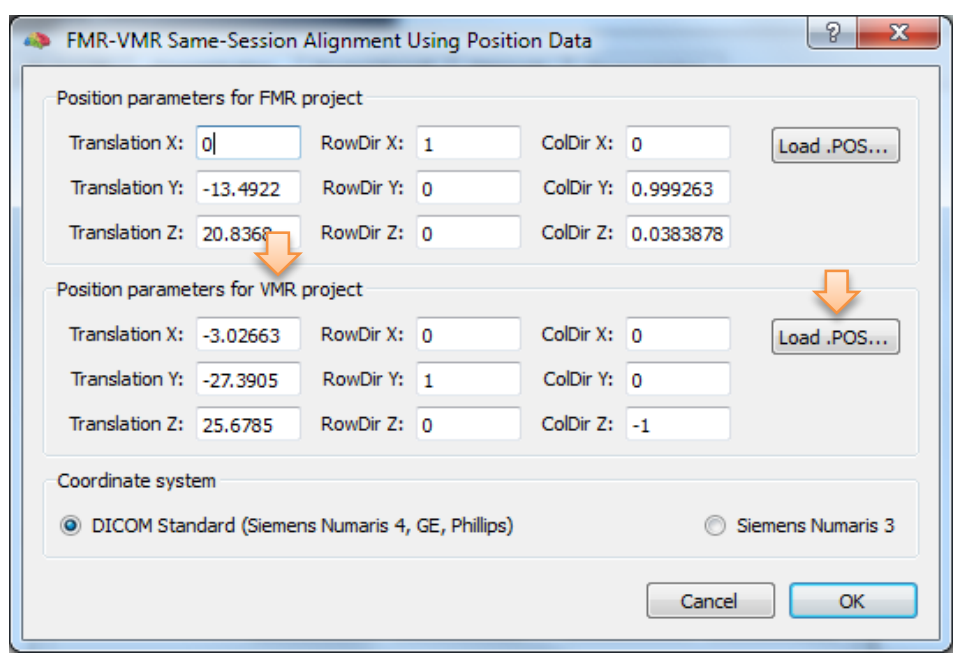

It will prompt this window to ask for the info.

The IA should work. Then do manual FA, adjusting only Translation and Rotation.

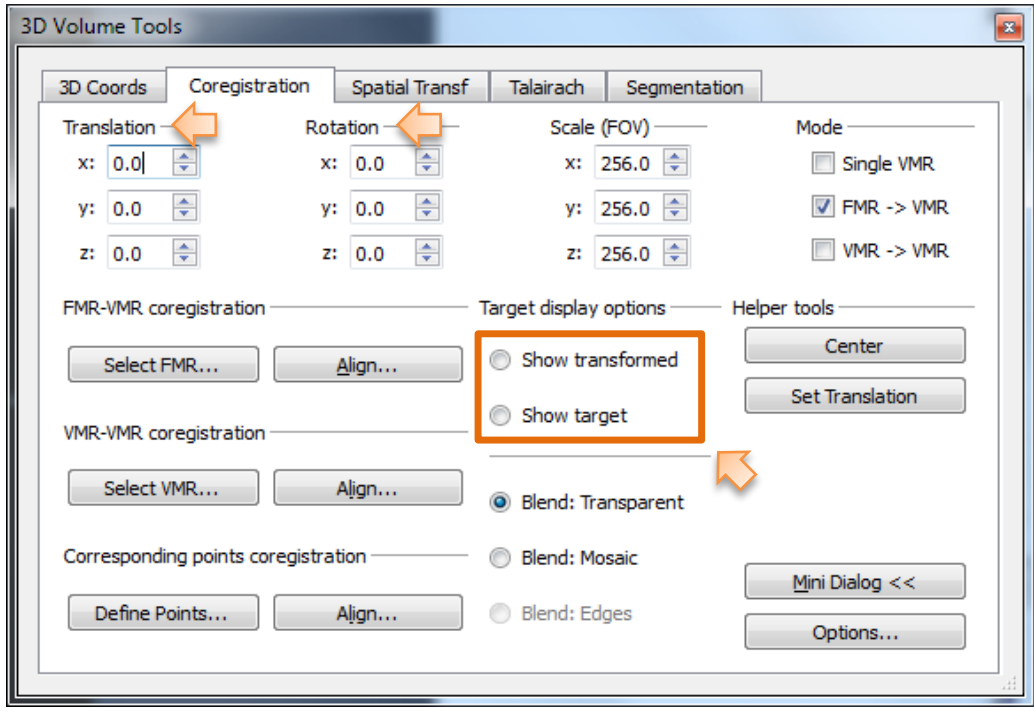

Better not to use Scale, since the same head in the scanner should be rigid, when the participant is not moving much. Even there is a distortion along the encoding direction, the scaling is not uniform across the brain. Scaling also increases difficulties in further alignments.

Also, better to use "Show transformed" \& "Show target" while aligning. Press F5 to switch between these two modes.

The Blend options actually shows you less differences between target and transformed brain.

Do the alignments for the rest of the .fmr files.

Now, an _IA.trf and a _FA.trf were created for each .fmr file. Use them to create the final VTC files.

Done. 


\section{- Optional:}

The .vtc files created in this way will likely to have a small translation, perhaps due to the matrix transformation computations (e.g. $\sim 0.5 \mathrm{~mm}$ in 3 axes for .vmrs in $1 \mathrm{~mm}$ isotropic resolution, $\sim 1 \mathrm{~mm}$ for .vmrs in $0.6 \mathrm{~mm}$ isotropic resolution). When you align the first fmr to the dummy vmr, the additional translations you have is usually the amount you need to correct for.

If you do want to correct for this, do the following:

Open the _FA.trf file in a text file editor (e.g. notepad), find the first 3 values in the last column. They are the amount of translations in 3 axes.

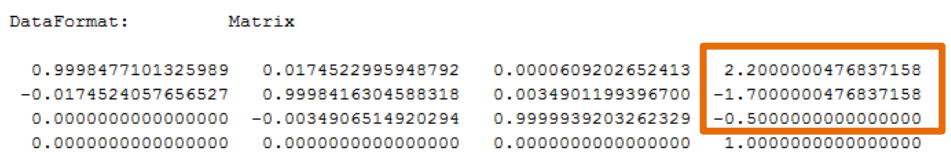

Subtract 0.5 from each value, and save the edited .trf file into a new file.

In BrainVoyager version 20 and above, you can switch to the matrix view directly in the Coregistration tab, Mode— and then Save .TRF in the Spatial Transf tab.
$\square$ Single VMR
FMR $\rightarrow$ VMR
VMR $\rightarrow$ VMR
TRS (C) Matrix

Use this modified_FA.trf file to create your .vtc file.

Done.

\section{Note, for MVPA searchlight, this 0.5 translation will change the result map.}

\section{For GLM the result is ok.}

The image below about transformation matrices in BVQX is from this document:

http://support.brainvoyager.com/documents/Volume Space/Coregistration/ManualRegistrationInBVQX v02.pdf

\section{AFFINE TRANSFORMATION MATRICES}

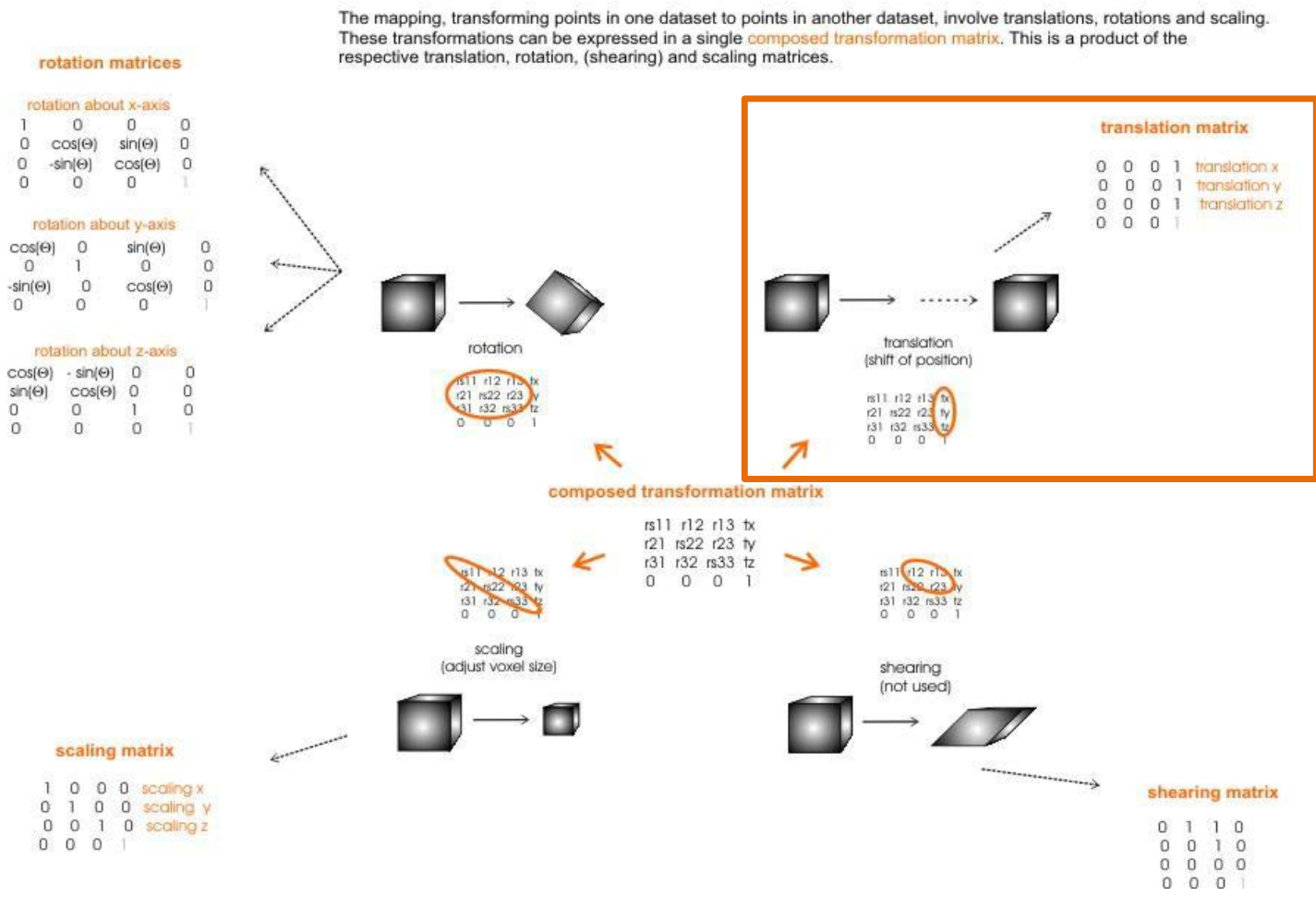




\section{- $\quad$ Check the alignment quality}

Whatever method you are using for alignments, it's advised to check the final alignments by loading each final vtc and click "Show VTC vol", do screenshots for each of them without moving the cursor, put all the screenshots in some image processing software (such as Photoshop), and toggle them on and off one by one (or make a .gif animation) to check the alignment.

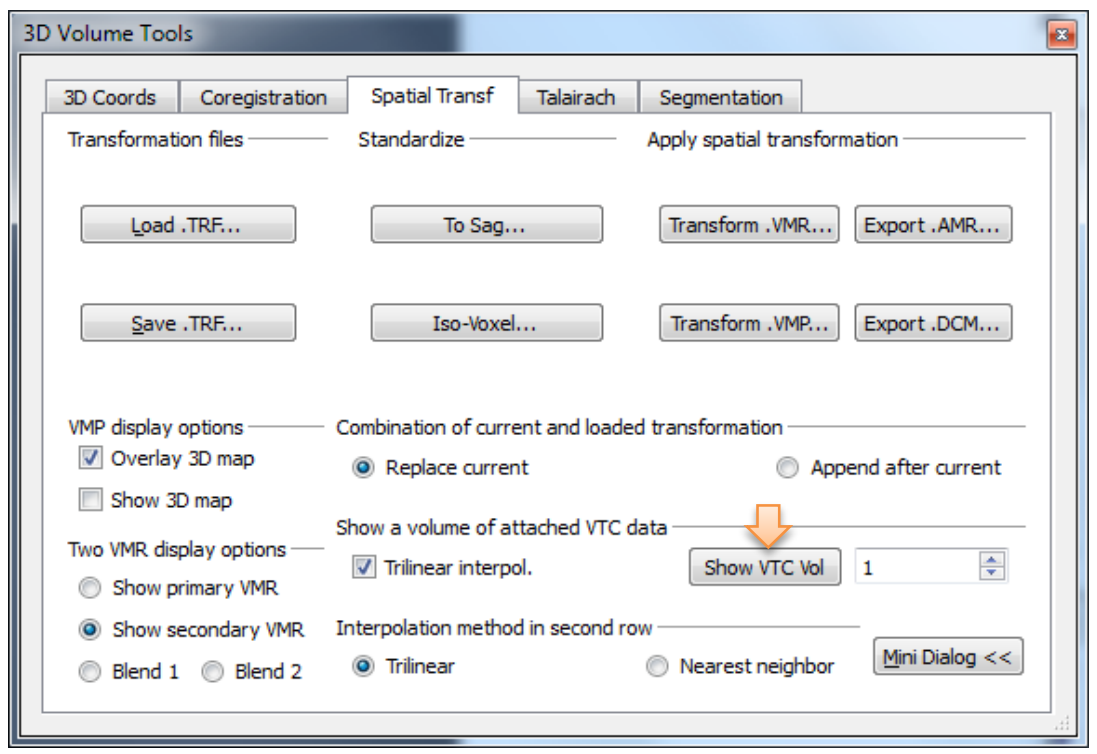

I recommend using the firstvol.fmr files do the alignments, because it's much faster to make the vtc (always with sinc interpolation) and check the quality, and do all subsequent adjustments such as realigning and re-making the vtcs. You can then go on creating the final vtc when you checked all the alignments and are happy with them.

When you want to adjust the previous alignment, you don't need to start from scratch, or take screenshots to copy values. After performing IA, you can always load the previous_FA.trf in the Spatial Transf tab (Load .TRF button), and go back to the Coregistration tab. The previous values would automatically be filled in there.

If you do 3D motion correction with a volume other than the first volume as the reference, then you have to do alignments with that volume, by creating a .fmr project with that volume only.

e.g. If you want to use the $9^{\text {th }}$ volume, create a project by skipping all the volumes before it and keep number of volumes as 1.

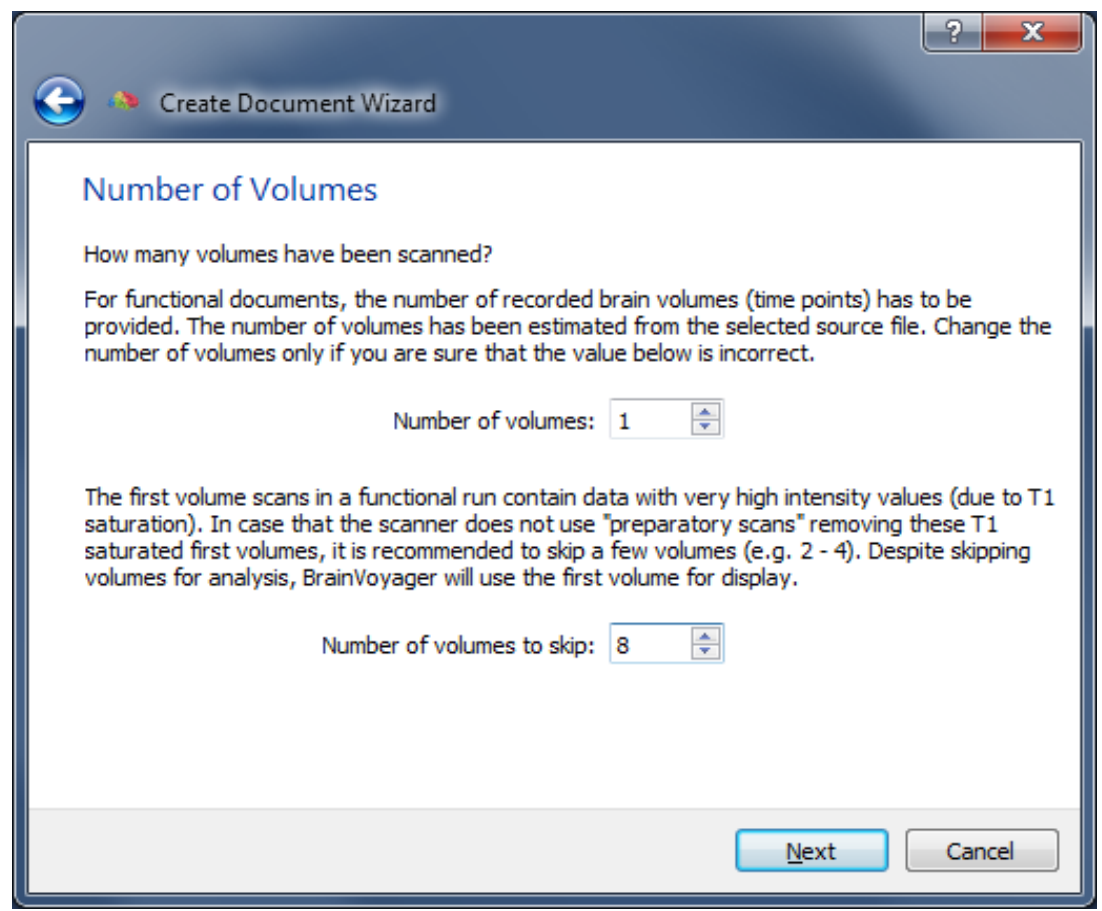


Acknowledgments 


\section{Acknowledgments}

First of all, I would like to thank my parents for their unconditioned support for me, to pursue an academic career so far away from home.

As my academic parents, both Bea and Rainer had helped me so much, in guiding me through my PhD trainings, and in connecting me to the next academic stage. Comparing to the attitudes of Chinese parents and teachers, one aspect that surprised me the most is: both of you did not discourage, but rather encouraged me to continue painting. Consequently painting became a mechanism of me to have a balanced mental health, and I will continue trying to make it a useful channel for valorization.

Bea, your open mind allowed me to go as far into the uncharted lands as I dared to. Without your generous support, it would not be possible for me to learn to use the 7T MRI machine, and to carry out so many 7T scans. For this alone I'm so grateful, not to mention all the other things you did for me. I've learned a lot and am still learning from you, for example the way you take in and combine new ideas together, and the way of how to tell a story both by a paper and by a talk. It's also so enjoyable to go to art exhibitions and have dinners with you. You've set a role model for me to combine art and science together, and showed me all kinds of valorization possibilities.

Rainer, 8 years ago you introduced me to the Research master program of cognitive neuroscience here; 6 years ago you introduced me to Bea; one year ago you helped me to stay longer to finish my PhD. Without you I wouldn't have been able to make the whole journey. I like very much your attention to details, and the most valuable thing I learned from you is: to be able to convince reviewers, I should first be able to convince all the coauthors.

I would also like to thank all the lab members; it was great to work with all of you. Kiki, I'm always amazed at how you can balance a two-kid family life with the tight research and teaching schedules. I've also learned a lot observing you setting up the Sensimetric system in the scanner, and how you interacted with the blind participants. It's great that you liked the Zongzi I made; I'Il make some once more in June. Tahnée, it's great that I could do some CFS studies with you, but I feel a bit sorry because CFS is such a bumpy ride...I like your calm attitude towards research, and your knitting, your cats, and all the cakes you made! It was also great fun staying with you and Rebecca during OHBM 2015. Rebecca, your way of cracking jokes is so unique! And it's so comfortable hanging out with you. Aline, I admire your VR and tactile studies, which both involved quite complicated setups. Your independence is also a joy to observe and learn from. Maarten and Jari, it was very nice to have you both as office neighbors and see your opinions from different perspectives.

Marta, you are currently the youngest PhD student in our lab. Teaching you how to analyze fMRI data was also a good training experience for me. It was nice to discuss all sorts of things with you, where I had the opportunity to introduce Chinese culture to you. With Sofia and Giulia we did the VR project together, which was a very busy but fun period of time. And with Charlotte and Bea we stayed in the castle, wandering in the forest and playing in the fog was so much fun! 
My thanks also go to former colleagues, and collaborators. Ruud and Lisanne, it was nice to work and hang out with you both. Although my facial EMG-CFS study didn't turn to fruition, thank you for your help in Tilburg! Mehrdad, you know so many people! It's amazing! And your lamb chops are delicious! Matteo and Alessia, it was nice to have some Italian influence from you. I still haven't found the chance to show you how to make dumplings, maybe someday in the future. Ron, great to discuss Chinese green tea with you! Ai, thank you for giving me the opportunity to do so many 7T scans for your project. It feels reassuring for me to have a pal (if I may) to discuss about academic careers for Asian female researchers. Anita, it's great to be there when you were working in our lab, and it's nice to talk to you now and then. Rick, Maria, Madita and Job, glad that we are carrying out our project alright. Let's continue to try to get nice data.

To some close non-lab-member colleagues: Miriam, glad to be the office-mate with you, in a calm environment. Yawen, thanks a lot to discuss everything with me! Let's also visit more places together!

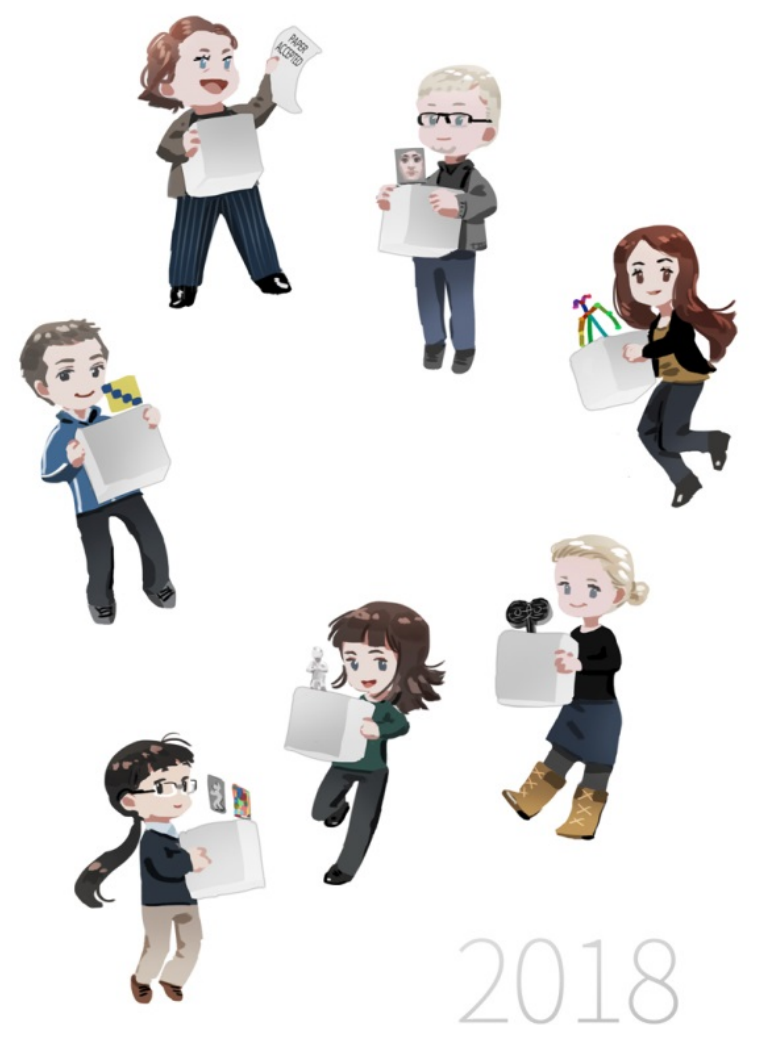

Figure 1. Lab members of the Brain and Emotion Laboratory, Maastricht University, 2018.

Now my thanks go to all the colleagues in the department. All of you are so helpful and friendly, and you all created an enjoyable research environment without too much pressure.

I thank all the colleagues who were teaching me in my research master years, including Bernadette, Elia, Milene, Alard, Alex, Bettina, Fren, Teresa, Lars, Michelle, Giancarlo, Peter. 


\section{Acknowledgments}

Peter, I felt I learned the most in your perception course. I wish there were more chances to learn more from you. Giancarlo, you are the role model of coding guru for me. I learned to code in MATLAB in your course, and I still benefit from the searchlight code you gave me a few years ago.

I also got great support from colleagues in my PhD years, especially for MR scanning. Federico, I learned from you every time you helped setting up the scanning sequences, both under $3 \mathrm{~T}$ and $7 \mathrm{~T}$, and the best part is that your explanations are very easy to understand and remember, which helped me so much. Valentin, thank you for your help in the 7T scans of Denmark participants, having you around when the 7T scanner was not working was super reassuring. Chris, you are always there quick to help, which I appreciate a lot. Kamil, you helped us a few times in scanning, and each time I learned something from you, an important one is: we should discard the bit of $\mathrm{fMRI}$ data when the visual task ends and the desktop is shown, because this bit is a big visual stimulation. Jan, you are amazing. It's an honor for me to paint the thesis cover for you.

My thanks go to the third-floor-mates. When I arrived here the third floor was very empty. Arko, Helen, Sanae, Mario, Franc, Dimo, Vittoria, Niels, Roy, Xu, Anna, Bert, Henk, seeing you inhabiting this quiet floor and chatting with you once in a while is very pleasant. To the colleagues of the other floors, my interactions with you were mainly in parties and during BHV trainings. Faruk, Marian, Ingo, Felix, Martha, and all colleagues I didn't manage to mention, chatting with you all was always very pleasant. And Sanne, Charlie, Selma, Kirsten, Linda, toast to the knitting club! And of course toast to Tahnée!

Last but not least, I want to thank all the secretaries. Christl, Riny, José and Eva, your support for everything, in all aspects, made my life a lot easier.

The research in this thesis was supported by the European Research Council, under the European Union's Seventh Frame-Work Programme (FP7/2007-2013)/ERC (grant numbers 295673 to Prof. Beatrice de Gelder, and 269853 to Prof. Rainer Goebel), by theEuropean Union's Horizon 2020 research and innovation programme under grant agreement No.645553 to Prof. de Gelder, and by the FPN-MBIC funding of Maastricht University to Minye Zhan and Prof. de Gelder.

For the help in collecting behavioral data included in this thesis, I would like to thank Sophia von Stockert, Giulia Barsuola, Joëlle Schroën, and Bibi Visser. 


\section{Publications}

\section{Peer-reviewed journal articles}

Hervais-Adelman, A., Legrand, L. B., Zhan, M., Tamietto, M., de Gelder, B., \& Pegna, A. J. (2015). Looming sensitive cortical regions without V1 input: evidence from a patient with bilateral cortical blindness. Front Integr Neurosci, 9, 51.

Poyo Solanas, M., Zhan, M., Vaessen, M., Hortensius, R., Engelen, T., \& de Gelder, B. (2017). Looking at the face and seeing the whole body. Neural basis of combined face and body expressions. Soc Cogn Affect Neurosci, nsx130-nsx130. doi: $10.1093 / \mathrm{scan} / \mathrm{nsx} 130$

Van den Stock, J., Tamietto, M., Zhan, M., Heinecke, A., Hervais-Adelman, A., Legrand, L. B., ... de Gelder, B. (2014). Neural correlates of body and face perception following bilateral destruction of the primary visual cortices. Front Behav Neurosci, 8, 30. doi: 10.3389/fnbeh.2014.00030

Zhan, M., \& de Gelder, B. (In press). Unconscious fearful body expression perception enhances discrimination of conscious anger expressions under continuous flash suppression. Neuropsychologia.

Zhan, M., Goebel, R., \& de Gelder, B. (2018). Ventral and Dorsal Pathways Differently Related to Visual Awareness of Body Postures under Continuous Flash Suppression. eNeuro, ENEURO. 0285-0217.2017.

Zhan, M., Hortensius, R., \& de Gelder, B. (2015). The Body as a Tool for Anger AwarenessDifferential Effects of Angry Facial and Bodily Expressions on Suppression from Awareness. PLoS One, 10(10), e0139768.

\section{Conference contributions}

Zhan, M., Goebel, R., Vaessen, M., de Gelder, B. (2017). Action categories are represented as distributed patterns in ventral and dorsal structures: $A$ high field and high resolution $\mathrm{fMRI}$ (7T) study. Nanosymposium presentation, Society of Neuroscience 2017.

Zhan, M., Hortensius, R., Goebel, R., de Gelder, B. (2015). Seeing invisible fearful bodies: A role of the intraparietal sulcus in non-conscious body processing. Annual meeting of ASSC.

Zhan, M., Hortensius, R., Goebel, R., de Gelder, B. (2015). Non-conscious processing of bodily emotion expressions in the dorsal stream. Annual meeting of OHBM. 


\section{Curriculum Vitae}

Minye Zhan was born on $28^{\text {th }}$ September 1984, in Hangzhou, China. She received her diploma for high school in 2003. After studying in Zhejiang University from 2003 to 2008, she received the Bachelor's degree for Basic Medical Sciences. To bridge the knowledge to cognitive neuroscience, she then spent two years as an intern in the Laboratory for Higher Brain Function, Institute of Psychology, Chinese Academy of Sciences, China. In 2010 she enrolled in the research master program of Cognitive Neuroscience in Maastricht University, the Netherlands, and graduated in 2012. From 2012 to 2017 Minye was a PhD student in the department of Cognitive Neuroscience, Faculty of Psychology and Neuroscience, under the supervision of Prof. Beatrice de Gelder and Prof. Rainer Goebel. In 2018 she does the postdoctoral work of 7T fMRI data acquisition for Prof. Goebel. From September 2018 she is working as a postdoctoral researcher in Neurospin, CEA, France, under the supervision of Prof. Stanislas Dehaene. In Minye's free time, she is a professional computer graphics (CG) painter. 